\title{
Adaptive neuromechanical control for energy-efficient and adaptive compliant hexapedal walking on rough surfaces
}

\author{
Dissertation \\ ZUR ERLANGUNG DES MATHEMATISCH-NATURWISSENSCHAFTLICHEN DOKTORGRADES \\ "DOKTOR RERUM NATURALIUM" DER GEORG-AUGUST-UNIVERSITÄT GÖTTINGEN
}

\author{
vorgelegt von \\ Xiaofeng Xiong \\ aus Hainan, China
}

Göttingen 2015 
Referentin/Referent: Prof. Dr. Florentin Wörgötter Koreferentin/Koreferent: Prof. Dr. Ulrich Parlitz Tag der mündlichen Prüfung: 08/06/2015 


\section{Abstract}

The control of multi-legged animal walking is a neuromechanical process where the nervous system produces neural signals to activate the muscles for driving the skeletons (e.g., legs and joints). To model and achieve this process on an artificial multi-legged machine is a difficult and challenging problem. This is because the process needs to model the interaction among the redundant nervous system (i.e., multiple neurons and synapses) and the redundant musculoskeletal systems (i.e., dozens of muscles and joints). Moreover, the two (i.e., neural and musculoskeletal) intrinsic redundancies lead to the kinematic redundancy where determining the joint angles that result in the particular positions at the endpoints of the legs is a tough task. The neurophysiologist Nikolai Bernstein pointed out, these three redundancies cause the difficulties of modeling and achieving a neuromechanical process on an artificial system with many degrees of freedom (DOFs). The modeled neuromechanical process needs in real time 1) to coordinate very many DOFs of jointed legs and muscle-like mechanisms, 2) to generate the proper leg stiffness (i.e., compliance), and 3) to determine joint angles that give rise to particular positions at the endpoints of the legs.

To show a way forward to model these three sub-processes, here we develop and implement neuromechanical controller coupled with sensorimotor learning on the hexapod robot AMOS with 19 DOFs. The controller consists of the modular neural network (MNN) for coordinating 18 joints and several virtual agonist-antagonist muscle mechanisms (VAAMs) for variable compliant joint motions. In addition, sensorimotor learning, including forward models and dual-rate learning processes, is introduced for predicting foot force feedback and for online adjusting the VAAMs' stiffness parameters. The control and learning mechanisms enable the hexapod robot AMOS to achieve adaptive compliant walking that adapts to different gaits (e.g., tripod gaits) and rough surfaces (e.g., gravel). As a result, AMOS can not only well classify rough surfaces, but also perform more energy-efficient walking on them, compared to other small legged robots. In addition, the developed method shows that the tight combination of neural control with tunable muscle-like functions, guided by sensory feedback and coupled with sensorimotor learning, is a way forward to better understand and solve adaptive coordination problems in multi-legged locomotion.

In this thesis, the presented work has seven key contributions: 1) it develops a simplified muscle model for the muscle-like functions that underlie variable compliant joint motions; 2) it presents the underlying muscle-like functions (e.g., brakes) of the VAAMs that are comparable to those of biological muscles; 3) it utilizes a proximodistal gradient of neural control and muscle functions to enhance the stability of legged robot locomotion under adaptive compliance control; 4) it achieves variable compliant

joint motions relying only on force sensing at the end effectors of the legs. Thus, the 
implementation reduces sensing and design efforts of legged robots; 5) it exploits the compliant joint signals generated by the VAAMs to well classify surfaces without using multiple sensing; 6) it utilizes sensorimotor learning to self-adjust the stiffness parameters of the VAAMs that adapts to insect-like gaits (e.g., tripod gaits) and surfaces (e.g., gravel), thereby leading to more energy-efficient hexapedal walking; 7) it provides a way forward to model stable and adaptive compliant insect-like walking, by implementing a bio-inspired neural network and several virtual agonist-antagonist mechanisms (VAAMs) on the hexapod robot AMOS with 19 DOFs (i.e., probing Bernstein's 'degrees of freedom' problem). 
Title Page . . . . . . . . . . . . . . . . . . .

Abstract ............................. ii

Table of Contents . . . . . . . . . . . . . . . . . . $\mathrm{v}$

Citations to Related Publications . . . . . . . . . . . . . . vii

Acknowledgments . . . . . . . . . . . . . . ix

Dedication . . . . . . . . . . . . . . . . $\mathrm{x}$

1 Introduction $\quad \mathbf{1}$

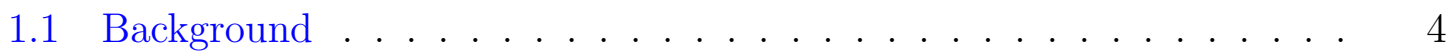

1.2 Goals . . . . . . . . . . . . . . . . . . . . . 7

1.3 Organization of the thesis . . . . . . . . . . . . 10

2 Related Methodologies and Robots 13

2.1 Neural control . . . . . . . . . . . . . . . . . . . . 13

2.2 Passive compliance control . . . . . . . . . . . . . . . . 16

2.3 Active compliance control . . . . . . . . . . . . . . . . 18

2.4 Legged robots . . . . . . . . . . . . . . . . . . 22

3 Biomechanical mechanisms for Variable Compliance Control 29

3.1 The insect-like robot AMOS . . . . . . . . . . . . . . . . 32

3.2 Virtual muscle-like mechanisms for variable compliance control . . . . 34

3.3 Variable compliance control for hexapedal standing and walking . . . 37

3.4 Variable compliant hexapedal standing against loads . . . . . . . . . 47

4 Neuromechanical Control for Variable Compliant Hexapedal Walking $\quad \mathbf{5 5}$

4.1 The modular neural network . . . . . . . . . . . . . . . 56

4.2 The neuromechanical principle . . . . . . . . . . . . . . . 59

4.3 Stable and variable compliant walking on five surfaces . . . . . . . . 65

4.4 Surface classification . . . . . . . . . . . . . . . . . . 70

4.5 The benefits of the neuromechanical controller . . . . . . . . . . . 74

5 Adaptive Neuromechanical Control for Online Compliance Adapta$\begin{array}{ll}\text { tion } & 77\end{array}$

5.1 Sensorimotor learning for adaptive compliant joint motions . . . . . . 80

5.2 Adaptive compliant joint motions for a gait and a surface . . . . . . . 82

5.3 Adaptive compliant joint motions for different gaits . . . . . . . . . . 86

5.4 Adaptive compliant joint motions for different surfaces . . . . . . . . 90

5.5 The compliant and efficient gaits for walking on the surfaces . . . . . 90 
6 Conclusions $\quad 95$

6.1 Summary of contributions . . . . . . . . . . . . . . . . 95

6.2 Future work . . . . . . . . . . . . . . . . . . . 98

$\begin{array}{lr}\text { A Appendix } & 117\end{array}$

A.1 Joint motion equation . . . . . . . . . . . . . . . . 117

A.2 Notations . . . . . . . . . . . . . . . . . . 117

A.3 Neuromechanical control of the legs . . . . . . . . . . . . . 118

A.4 The tripod gaits of AMOS and an insect . . . . . . . . . . . . 120

$\begin{array}{lr}\text { B Curriculum Vitae } & 121\end{array}$ 


\section{Citations to Related Publications}

Some portion of Chapter 3 is based on the following three papers:

Xiong, X., Wörgötter, F., and Manoonpong, P. (2013). A neuromechanical controller of a hexapod robot for walking on sponge, gravel and snow surfaces. In Advances in Artificial Life. Proceedings of the 11th European Conference on Artificial Life ECAL 2013, pages 989-996, Taormina, Italy.

Xiong, X., Wörgötter, F., and Manoonpong, P. (2013). A simplified variable admittance controller based on a virtual agonist-antagonist mechanism for robot joint control. In Proc. Intl Conf. on Climbing and Walking Robots CLAWAR, pages 281-288, Sydney, Australia.

Xiong, X., Wörgötter, F., and Manoonpong, P. (2014). Virtual agonistantagonist mechanisms produce biological muscle-like functions: An application for robot joint control. Industrial Robot: An International Journal, $41(4): 340-346$.

Chapter 4 appears in its entirety as

Xiong, X., Wörgötter, F., and Manoonpong, P. (2014). Neuromechanical control for hexapedal robot walking on challenging surfaces and surface classification. Robotics and Autonomous Systems, 62(12):1777 - 1789.

Finally, most of Chapter 5 was submitted to IEEE Tran. on Cybernetics

Xiong, X., Wörgötter, F., and Manoonpong, P. (2015). Adaptive and Energy Efficient Walking in a Hexapod Robot under Neuromechanical Control and Sensorimotor Learning. IEEE Transactions on Cybernetics. 


\section{Acknowledgments}

First of all I would like to thank my supervisors Prof. Dr. Florentin Wörgötter, Prof. Dr. Ulrich Parlitz, and Porf. Dr. Poramate Manoonpong for guiding me through my work by sharing their experiences with me and for countless hours of fruitful discussions and patient corrections without which this work would not have been successfully done. The discussions and corrections are also done from Frank Hesse, Martin Biehl, Dennis Goldschmidt, Sakyasingha Dasgupta, Michael Fauth, Jeremie Papon, Timo Nachstedt, and Prof. Dr. Tomas Kulvicius, so I am very thankful for their efforts, too. An especially big thanks to Ursula Hahn-Wörgötter who always and patiently helps me out in any way that she could.

Secondly, I would like to thank all my colleagues and friends for their direct and/or indirect input to my work and a having great time together. Many thanks go to Guoliang Liu, Qi Yao, Jan-Matthias Braun, Subhi Shaker Barikhan, Mohamad Javad Aein, Alexey Abramov, Yijing Liu, Simon Reich, Markus Schoeler, Irene Markelic, Minija Tamosiunaite, Yinyun Li, Christian Tetzlaff, Christoph Kolodziejski, HarmFriedrich Steinmetz, Kolja Riefsdahl, Patrick Kesper, Alejandro Agostini, Eren Erdal Aksoy, Steffen Zenker, Eduard Grinke, Lei Zhou, Guanjiao Ren, Yuichi Ambe, and Johannes Widenka. Special thanks go out to Prof. Dr. Jianwei Zhang and Prof. Dr. Ying $\mathrm{Hu}$ who give me a lot of suggestions on my research career.

And last but not least, I would like to thank my father Shan Xiong, my mother Ruruo Zheng, and my sister Juanjuan Xiong without whom I would not have achieved all that in my life what I have now. 
Dedicated to my father Shan Xiong, my mother Ruruo Zheng, and my sister Juanjuan Xiong. 


\section{1 \\ Introduction}

Legged animal locomotion arises from an intricate interplay between the control of the nervous system and the musculoskeletal systems (Miller et al., 2012; Nishikawa et al., 2007; Tytell et al., 2011). This interplay has emerged from a neuromechanical control system where the nervous system produces neural signals to activate the muscles for driving the skeletons (e.g., legs and joints). Neuromechanical control governs how legged animals achieve adaptive locomotion on different surfaces (Abbas and Full, 2000; Chiel et al., 2009). For example, cockroaches rely more on their musculoskeletal systems to move over a regular surface. Whereas moving over a more difficult one, they need to resort to the integrations of their nervous systems and musculoskeletal systems (Sponberg and Full, 2008). Therefore, modeling neuromechanical control refers to a computational model of the interaction among the redundant nervous system (i.e., multiple neurons and synapses) and the redundant musculoskeletal systems (i.e., many muscles and joints). In a cockroach (e.g., Blaberus discoidalis), for instance, there are millions of neurons, 220 muscles, and more than 19 degrees of freedom (DOFs) that contribute to its locomotion (Full and Ahn, 1995; Nishikawa et al., 2007). Moreover, the two (i.e., neural and musculoskeletal) intrinsic redundancies lead to the kinematic redundancy where determining the joint angles that result in the particular positions at the endpoints of the legs is a tough task. Due to the three redundancies, modeling the cooperations within and between the functional (e.g., musculoskeletal) components of the neuromechanical controller is a very challenging task (i.e., Bernstein's famous 'degrees of freedom' problem) in legged locomotion (Bernstein, 1967; Nishikawa et al., 2007).

Generally, legged locomotion requires the modulation of leg stiffness to accommodate different gaits (Nishi, 1998; Nishii, 2000) and surfaces (Spence et al., 2010; Farley et al., 1998; Ferris et al., 1998), thereby leading to more energy-efficient walking on diverse surfaces (Spence, 2011). The leg stiffness $K_{\text {leg }}$ can be virtually quantified by the ratio of the normalized impact force $F_{\text {ext }}$ to the normalized displacement $\Delta L_{\text {leg }}$ of the center of mass (COM) (Blickhan and Full, 1993; Dudek and Full, 2006) (see Fig. 1.1 (a)). In the quantifications, animal locomotion is modeled by the spring-loaded in- 
verted pendulum $^{1}$ (SLIP) where the COM is supported by a mass-less elastic leg with spring stiffness (see Fig. 1.1 (b)) (Altendorfer et al., 2001; Seipel, 2005; Altendorfer et al., 2004). Quantitatively, the inverse of the leg stiffness $K_{\text {leg }}$ is the leg compliance $Y_{l e g} . Y_{l e g}$ is usually used to quantify the leg compliance of the legged robots under compliance control (Hyun et al., 2014; Bosworth et al., 2014; Sreenath et al., 2010).

$$
K_{\text {leg }}=\frac{F_{\text {ext }}}{\Delta L_{\text {leg }}}, Y_{\text {leg }}=\frac{1}{K_{\text {leg }}} .
$$

(a)

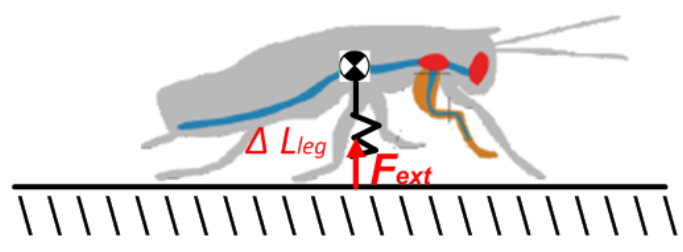

(b)

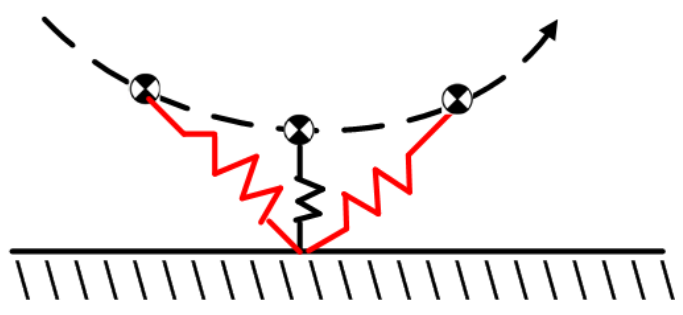

Figure 1.1: Virtual leg stiffness and spring-loaded inverted pendulum. (a) The virtual leg stiffness $K_{\text {leg. }}$. Quantitatively, the inverse of stiffness $K_{\text {leg }}$ is compliance $Y_{\text {leg }}$. (b) The spring-loaded inverted pendulum (SLIP). The COM is supported by a mass-less elastic leg with spring stiffness.

In multi-legged robots, their compliant joint motions and coordinated leg patterns (i.e., gaits) emerge from the tunable muscle-like mechanisms and the neural network that together constitute a neuromechanical controller. The adaptiveness of the neuromechanical controller can be achieved by sensorimotor learning (Smith et al., 2006; Wolpert et al., 2011) that enables the passive properties (e.g., stiffness parameters) of the muscle-like mechanisms to adapt to diverse gaits and surfaces. The adaptive neuromechanical controller provides a way forward to embed a physical template model into a computable anchor model. A template represents the simplest model of the locomotion by trimming away the detailed descriptions (e.g., muscles) of the degrees of freedom (Lee et al., 2008b). However, the template must be anchored to a representative model (i.e., anchor) by describing a nervous system, muscles, joints, and legs (Koditschek et al., 2004). This is because these components conspire with the external environments to produce complex and adaptive movements in legged animal locomotion (Tytell et al., 2011). The template and anchor are two dynamical models (Full and Koditschek, 1999), which propose a specific solution to Bernstein's famous 'degrees of freedom' problem (Bernstein, 1967). As Bernstein pointed out, the need to control many degrees of freedom (DOFs) is a characteristic of neuromechanical

\footnotetext{
${ }^{1}$ In Fig. 1.1 (a), the schematic diagram of the cockroach is modified from Spence (2009).
} 


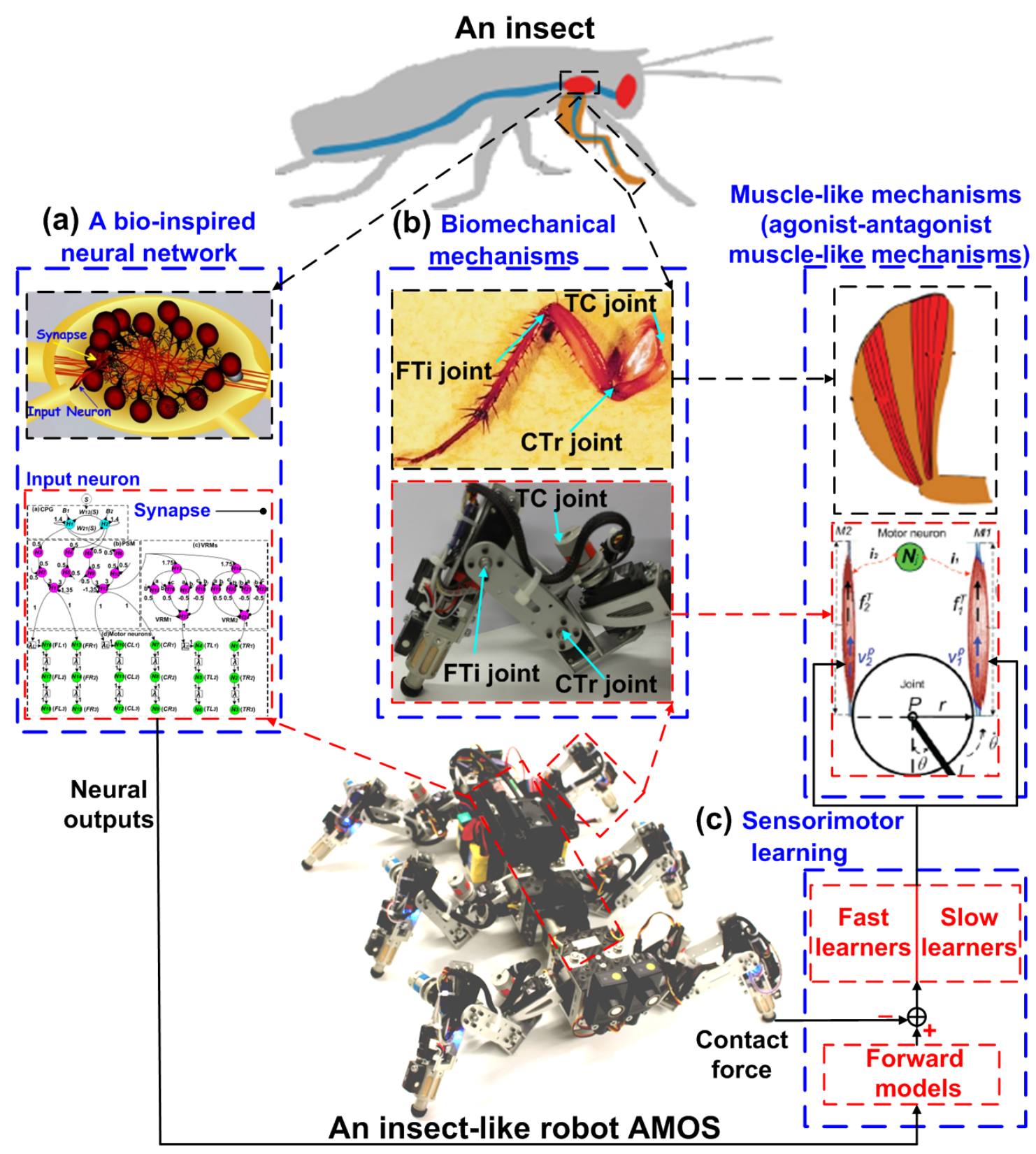

Figure 1.2: An adaptive neuromechanical controller for stable and adaptive compliant insect-like walking. (a) A bio-inspired neural network for coordinated joint motions (i.e., gaits). (b) Biomechanical components for variable compliant joint motions. (c) Sensorimotor learning for adaptive compliant joint motions.

systems (Bernstein, 1967; Nishikawa et al., 2007). To our knowledge, most current neuromechanical models (i.e., anchors) are computationally expensive and not practical for being implemented on physical legged robots. Therefore, a neuromechanical 
controller for adaptive and energy-efficient physical legged locomotion that accommodates different gaits and surfaces remains an important and unresolved problem (Holmes et al., 2006; Nishikawa et al., 2007).

To solve this problem, we develop an adaptive neuromechanical controller on an insect-like robot AMOS with 19 DOFs (see Figs. 1.2). The controller consists of a bio-inspired neural network ${ }^{2}$, several biomechanical mechanisms ${ }^{3}$ (i.e., joints and muscle-like mechanisms), and sensorimotor learning. The adaptive compliant joint motions of AMOS are achieved by actively adjusting the passive properties (e.g., stiffness parameters) of the muscle-like mechanisms driving its joints. Such adjustments are achieved by sensorimotor learning that consists of the forward models and dualrate (i.e., fast and slow) learners (see Fig. 1.2 (c)). As a result, AMOS can online adjust the stiffness parameters to produce adaptive compliant joint motions, thereby accommodating its walking to different gaits and surfaces. Such joint motions enable AMOS to not only classify rough surfaces (e.g., gravel), but also achieve adaptive and energy-efficient walking over different surfaces with the appropriate gaits. Generally, energy efficiency is measured by cost of transport ${ }^{4}$ (COT, i.e., specific resistance (Gregorio et al., 1997; Saranli et al., 2001)). Lower COT corresponds to more energyefficient locomotion. We show that our hexapod robot AMOS can achieve lower COTs than other small legged robots (less than $8 \mathrm{~kg}$ (Galloway, 2010)), when proper gaits are chosen for walking over different surfaces. These surfaces include gravels (e.g., coarse gravel), snow, an elastic and soft sponge (stiffness $0.523 \mathrm{kN} / \mathrm{m}$ ), and muddy grassland. In the next section, the background of studying and understanding legged locomotion is described, which is followed by a description of basic models of legged locomotion. This chapter concludes with an overview of the organization of the thesis.

\subsection{Background}

Attempts to study and understand the principles underlying legged locomotion have been ongoing for thousands of years. In 350 B.C., the great Greek philosopher and scientist Aristotle proposed a list of questions in a text On the Gait of Animals (Aristotle et al., 1912). For example, one of the listed questions is that why men and birds are bipeds, but fishes legless. His answer to the question is that men and birds are bipeds because they have superior parts, which are distinguished from front parts. However, he studied these empirical questions based on teleological presumptions, rather than scientific methods (e.g., mathematics or anatomy). During the Renais-

\footnotetext{
${ }^{2}$ In Fig. 1.2 (a), the schematic diagram of a ganglion of the insect nervous system is modified from the tutorial video presented by Larry Keeley

${ }^{3}$ In Fig. 1.2 (b), the schematic diagram of insect muscles is modified from Spence (2009).

${ }^{4}$ The cost of transport (i.e., COT) quantifies the energy efficiency transporting an animal or a vehicle from one place to another. It is also called specific resistance.
} 
sance, the great Italian painter and mathematician Leonardo Da Vinci studied the structures of animal bodies and identified muscles and nerves using anatomical and kinesiological methods. He also built legged robots (see Figs. 1.3 (a) and (b)) to form the bridge between his mechanical work and anatomical studies (Mario, 2008). Other significant contributions can be found in On the Movement of Animals by great Italian physicist and mathematician Giovanni Alfonso Borelli, who is often credited as the father of biomechanics. He argued that legged locomotion is similar to machinery movements based on mechanical laws, which are proven by utilizing geometry in his manuscripts (see Figs. 1.3 (c) and (d)). Furthermore, Borelli first suggested that muscles do not exercise any vital movements other than by contracting.
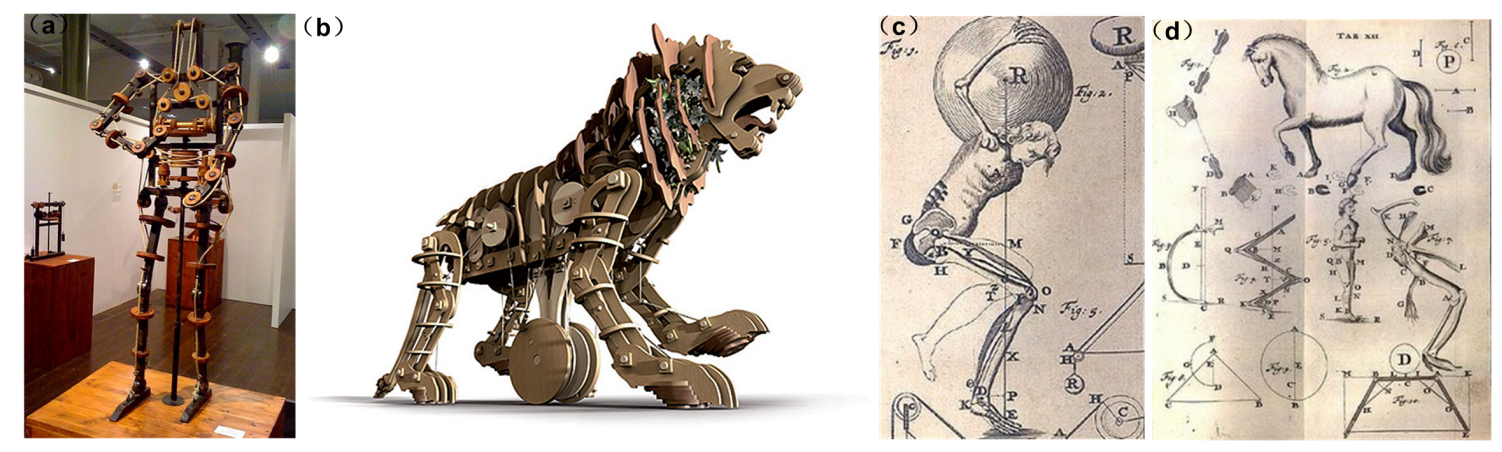

Figure 1.3: The legged robots developed by Leonardo Da Vinci and the manuscripts on leg biomechanics by Giovanni Alfonso Borelli. (a) A two-legged robot. (b) A fourlegged robot. For more details on these robots, see Mario (2008). (c) Two-legged standing biomechanics (d) Four-legged locomotion biomechanics. For more details on these biomechanical models, see Borelli and Maquet (2014).

In the 20th century, the great Russian physiologist Nikolai Bernstein noted that legged locomotion is an intricate interplay requiring the functions of the nervous system, muscles, tendons, and joints. Such an interplay is a neuromechanical interaction that governs how legged animals with many DOFs achieve adaptive locomotion on different surfaces (Abbas and Full, 2000; Chiel et al., 2009). Owing to many DOFs, modeling the cooperations within and between different functional components of neuromechanical systems in legged locomotion is a very challenging task. As Bernstein pointed out, many degrees of freedom (DOFs) is a characteristic of neuromechanical systems (Bernstein, 1967). Along this paradigm, Full and Koditschek proposed a specific solution where two dynamic models (i.e., so-called templates and anchors) are used to model legged locomotion with many DOFs (Full and Koditschek, 1999). A template represents the simplest model of the locomotion by trimming away the detailed descriptions (e.g., muscles and joints) of the degrees of freedom (see Fig. 1.4 (a)). Whereas an anchor model is a representative model describing a nervous system, muscles, joints, and legs with many DOFs like in insects (Holmes et al., 2006) 
(see Fig. $1.4(\mathrm{~b})$ ). Consequently, there are two major methodologies to model and control legged locomotion, which follow the concepts of the template and the anchor.

(a) Template

Simple conceptual model

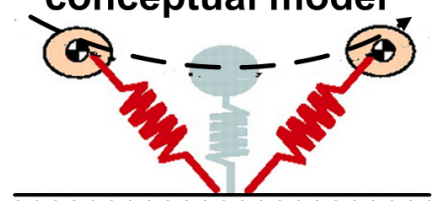

111111111111111111

Reduce dimensions by trimming away complexity (b) Anchor $\frac{1}{0} 00$
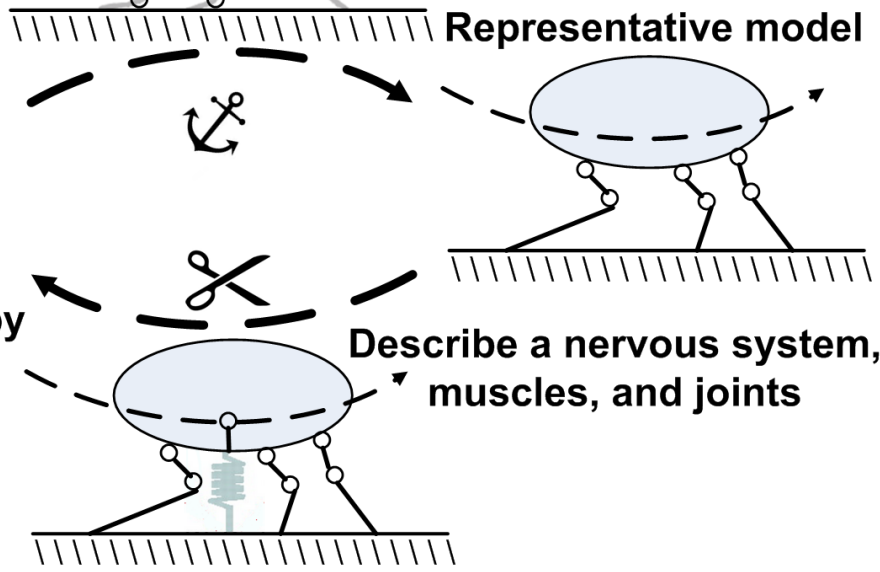

Figure 1.4: Two ways of modeling legged locomotion : a template and an anchor (Full and Koditschek, 1999; Koditschek et al., 2004). (a) The template. The template represents the simplest conceptual model of legged locomotion by reducing the complexity. The most general template for legged locomotion is the spring-loaded inverted pendulum (SLIP). (b) The Anchor. The anchor is the representative model describing a nervous system, muscles, joints, and legs with many DOFs like in insects (Holmes et al., 2006). Note that the figure refers to Fig. 6 in Koditschek et al. (2004).

The most general template for legged locomotion is the spring-loaded inverted pendulum (SLIP) (Altendorfer et al., 2001; Seipel, 2005; Altendorfer et al., 2004). The SLIP is a very simple conceptual model of legged locomotion where the center of mass $(\mathrm{COM})$ is supported by a mass-less elastic leg with spring stiffness (see Fig. 1.4 (a)). No damping is included, since the SLIP model assumes that there are the massless feet and lossless collisions. The SLIP model states that a stance leg behaves like an inverted pendulum, is thus energetically conservative. When the foot touches the ground, gravity and force produced by the spring will conspire to act on the COM. Whereas, the leg in swing phases (i.e., in the air), the COM performs a descending acceleration due to gravity. Owing to its simplicity, the SLIP model helps researchers easily understand the fundamentals of legged locomotion and apply them to develop legged robots (Altendorfer et al., 2001, 2004). For example, the hexapod robots (i.e., RHex robots) were designed by Koditschek and his colleagues (Saranli et al., 2001; Koditschek et al., 2004). Each RHex robot having only six DOFs showed unprecedented mobility over different surfaces. Besides, they can also achieve energyefficient locomotion by exploiting passive variable compliant legs. For example, leg compliance of a RHex robot (i.e., EduBot) was manually tuned to adapt its running 
speeds based on energetic cost (Galloway et al., 2011). The RHex robot is the best example of a coordination architecture controlling faster movement (e.g., running), where mechanical properties (e.g., leg compliance) must be increasingly well tuned to adapt to different environments (Koditschek et al., 2004; Nishikawa et al., 2007). In such a case, more feed-forward and decentralized control can suffice, since feedback control may not be effective due to noisy sensing. By contrast, slower movement (e.g., walking) can count heavily on feedback allowing for more adaptive insect-like movement where force feedback is of great importance (Kaliyamoorthy et al., 2005). Similarly, here a more feedback and centralized coordination architecture will be utilized to control our insect-like robot AMOS in a neuromechanical manner (Xiong et al., 2014a, 2013). Moreover, the modeling of RHex robots is no more than a template, since this template behavior was not embedded within a detailed model (i.e., anchor). The anchor model is a representative model describing a nervous system, muscles, joints, and legs with many DOFs like in insects (Holmes et al., 2006; Koditschek et al., 2004). There should be a natural embedding of the template behavior within the anchor (Full and Koditschek, 1999; Lee et al., 2008b), since templates and anchors are more than 'simple models' and 'complex models' .

Attempting to embed the template within an anchor, Holmes et. al. (Holmes et al., 2006) presented mathematical models of legged locomotion, which integrate a nervous system including central pattern generators (CPGs), muscle dynamics, and body mechanics. Based on these models, a neuromechanical model containing up to 270 ordinary differential equations was proposed for describing bursting neurons in a CPG (Kukillaya et al., 2009). Another neuromechanical model was applied to a single degree of freedom to simulate insect locomotion (Proctor and Holmes, 2010). The joint was actuated by a pair of agonist and antagonist muscles driven by motoneurons and a CPG in computer simulations. A Hill muscle model (Hill, 1938; Zajac, 1989) was adopted to express the force generated by agonist and antagonist muscles where there are up to 16 parameters to be tuned. These detailed neuromechanical models (i.e., anchors) are too computationally expensive to be implemented on physical legged robots. Therefore, a computational neuromechanical model for adaptive and energy-efficient physical robot locomotion that adapts to different gaits and surfaces remains an important and unresolved problem in a neuromechanical context (Holmes et al., 2006; Nishikawa et al., 2007).

\subsection{Goals}

The above section shows that embedding a template model within an anchor controller remains an important and unsolved problem for physical legged locomotion with many DOFs. Solving this problem can bridge the gap from the template to the anchor (Full and Koditschek, 1999) (see Figs. 1.4), which provides a specific solution to Bernstein's famous 'degrees of freedom' problem (Bernstein, 1967) in legged loco- 
motion. To do so, the solution needs to not only model a modular nervous system and the musculoskeletal systems, but also reproduce intricate interactions between them. Generally, the modular nervous system produces signals to activate extensor and flexor muscles that coordinate multi-jointed legs. In addition to the coordination, leg stiffness should be actively tuned by altering the stiffness of the muscles that adapts to diverse surfaces and gaits. One of the important motivations behind these behaviors is that tunable leg stiffness allows legged animals to achieve energy-efficient locomotion over the surfaces (Spence, 2011). For example, bipeds (e.g., humans) are capable of adjusting their leg stiffness to accommodate the surfaces with variable substrate properties (Ferris et al., 1998; Farley et al., 1998), thereby leading to adaptive and energy-efficient locomotion (Spence, 2011; Kerdok et al., 2002). Polypeds (e.g., cockroaches) also tune their leg stiffness to different surfaces and gaits based on energetic cost (Full et al., 2000; McMahon and Cheng, 1990; Nishii, 2000; Nishi, 1998). Neurophysiological studies have revealed that these behaviors arise from the interplay between the modular nervous system and the musculoskeletal systems (e.g., muscles and legs) of legged animals (Miller et al., 2012; Nishikawa et al., 2007). The nervous and musculoskeletal systems do not isolatedly function in adaptive and energy-efficient legged locomotion (Tytell et al., 2011). Isolating them, for example, may lead to energy inefficiency of legged locomotion (Manoonpong et al., 2013b; Xiong et al., 2014a).

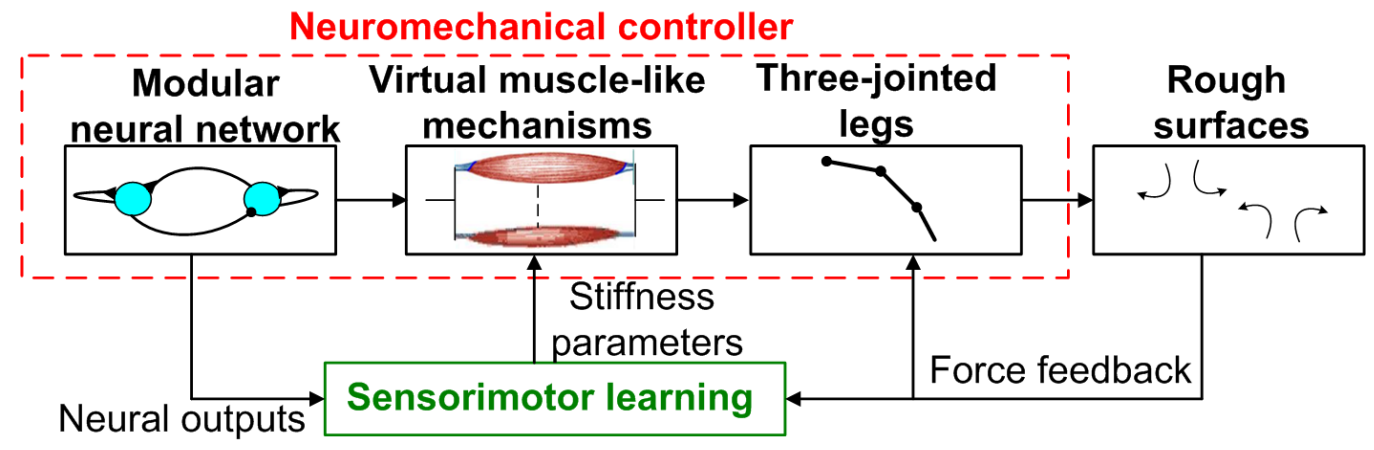

Figure 1.5: Neuromechanical control coupled with sensorimotor learning applied to physical robot locomotion. The neuromechanical controller consists of a modular neural network, several virtual muscle-like mechanisms, and the three-jointed legs. The neural network produces the neural outputs to activate the virtual muscle-like components driving the legs. Then the legs interact with rough surfaces (e.g., gravel), which produces physical force feedback. In addition, sensorimotor learning predicts force feedback based on the neural outputs. Taking physical and predicted force feedback as the inputs, the sensorimotor learning online changes the stiffness parameters of the virtual muscle-like components that enables the driven joints to achieve adaptive compliant motions. Such joint motions adapt to different surfaces and insect-like gaits. 
Therefore, in this thesis, the adaptive neuromechanical controller (see Fig. 1.5) shows a way forward to model redundant legged locomotion and implement the model on our hexapod robot AMOS with 19 DOFs. Specifically, each joint of AMOS is driven by a pair of the virtual muscle-like components, which is activated by an output of a modular neural network. Virtual means that the joint, physically driven by a standard servo motor, produces variable compliant motions as if it were driven by a pair of agonist and antagonist muscles. The adaptiveness of such joint motions is achieved by sensorimotor learning that actively tunes the stiffness parameters of the virtual muscle-like mechanisms driving the joints. Such active tuning enables AMOS to achieve adaptive compliant joint motions that adapt to various surfaces and insect-like gaits. The insect-like gaits of AMOS (e.g., a tripod gait (Ritzmann and Zill, 2013), see Figs. 1.6 and A.1) result from the modular neural network of the neuromechanical controller.
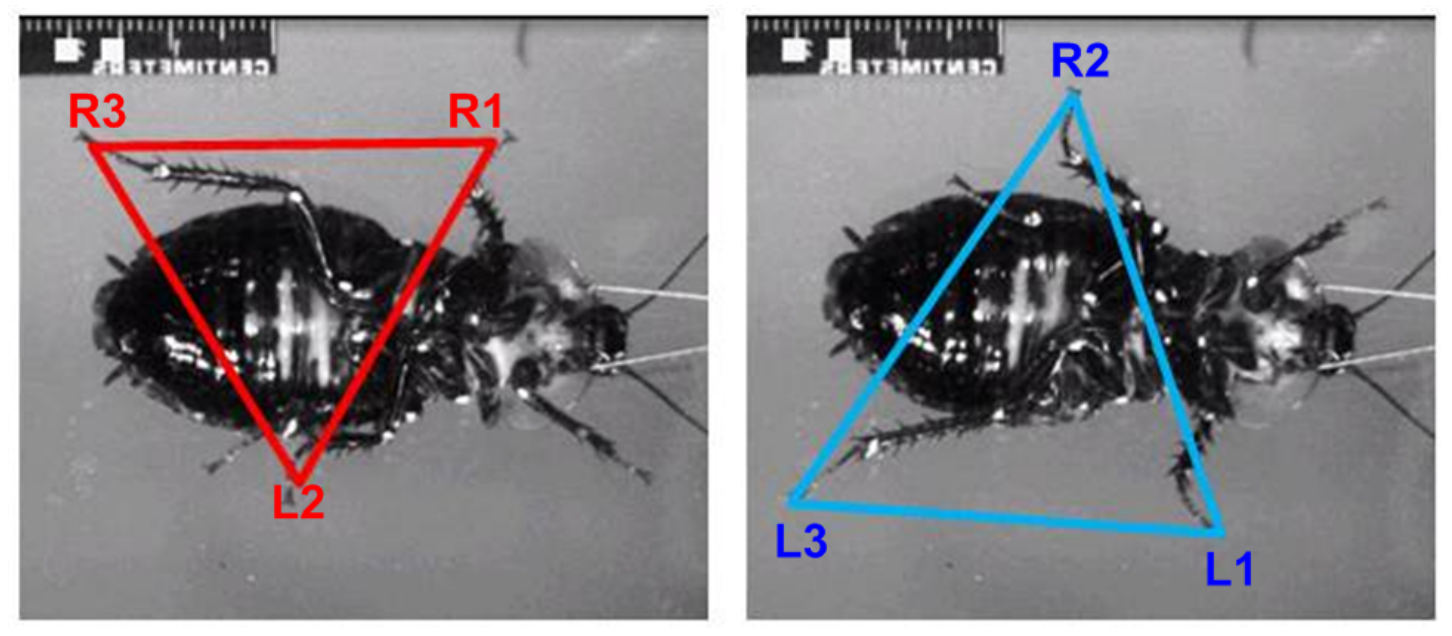

Figure 1.6: A tripod gait pattern for a tethered insect (modified from Ritzmann and Zill (2013)). Two video snapshots show extreme rear position during stance of the two tripods of legs. Abbreviations are: $\mathrm{R}(1,2,3)=$ Right (Front, Middle, Hind) leg, $\mathrm{L}(1,2,3)=$ Left (Front, Middle, Hind) leg.

The adaptive neuromechanical controller can reduce design efforts and structural complexities of bulkier and compliant legged robots, which are generally driven by physical artificial muscles (Kingsley et al., 2006) or variable impedance actuators (Ham et al., 2009). These components or actuators are difficult to apply to control of small legged robots (less than $8 \mathrm{~kg}$ ), such as our hexapod robot AMOS (5.4 kg). In addition, the controller utilizes a proximo-distal gradient found in neural control and muscle functions of legged animal locomotion (Nishikawa et al., 2007; Daley and Biewener, 2011, 2006), to enhance locomotor stability of the legged robot on rough terrain (e.g., gravel). It shows a novel way to deal with locomotor instabilities, which intrinsically result from active compliance control. Moreover, online self-adjusted 
compliance control to a physical legged robot with many DOFs remains an unsolved problem in the context of energy-efficient waking on different surfaces. Specifically, online adjustment of stiffness parameters in joint compliance control is a difficult problem in the context of energy-efficient legged locomotion with many DOFs (Garcia and de Santos, 2006). To solve the problem, sensorimotor learning is utilized for online adjusting the stiffness parameters of the muscle-like components that leads to adaptive compliant joint motions. Such motions enable AMOS to adapt its walking to different gaits and surfaces, thereby leading to energy-efficient walking on rough surfaces (e.g., gravel). In addition, the adaptive compliant motions allow AMOS to classify the surfaces well.

\subsection{Organization of the thesis}

The rest of the thesis is as follows: Chapter 2 will briefly introduce related methodologies (e.g. active compliance control) and discuss their inherent and unresolved problems, which can be solved using our proposed method (see Fig. 1.5). In our method, a modular neural network, several muscle-like mechanisms, a bio-inspired body, and sensorimotor learning act together to achieve stable and adaptive compliant walking of our hexapod robot AMOS.

In Chapter 3 we will show AMOS's biomechanical components including a bioinspired body and the virtual muscle-like components. Emulating a cockroach, its body has six legs, each of which consists of a TC (Thoraco Coxal) joint allowing forward and backward motions, a CTr (Coxa Trochanteral) joint allowing elevation and depression motions, and an FTi (Femur Tibia) joint allowing extension and flexion motions. Each joint is driven by a pair of the muscle-like mechanisms (i.e., the virtual agonist-antagonist mechanisms, the VAAMs). Changing the stiffness and damper parameters of the VAAMs produces variable compliant joint motions. Underlying such motions, the functions (e.g., the brake (Ahn and Full, 2002; Dickinson et al., 2000)) of the VAAMs are comparable to those of biological muscles.

Further on, in Chapter 4, we will present a biologically-inspired modular neural network consisting of a central pattern generator (CPG), a phase switch module (PSM), and two velocity regulating modules (VRMs). The neural network produces the 18 output signals to activate the 18 pairs of the VAAMs, which coordinate AMOS's six three-jointed legs. Subsequently, a proximo-distal gradient (Nishikawa et al., 2007; Daley and Biewener, 2011, 2006) is applied to neuromechanical control of our hexapod robot AMOS. Such a gradient enhances the stability of its variable compliant walking on rough surfaces (e.g., gravel). Here the variable compliant walking is achieved by manually choosing the stiffness parameters of the VAAMs. The well-chosen parameters and an efficient insect-like gait (i.e., a slow wave gait) enable AMOS to achieve more energy-efficient locomotion, compared to mere neural control (Manoonpong et al., 2013b). 
And finally, online self-adjusting stiffness parameters of the VAAMs is achieved by sensorimotor learning that will be presented in Chapter 5 . Such online selfadjustments make AMOS accommodate its variable compliant walking to different surfaces and gaits (e.g., a tripod gait). As a result, AMOS can achieve more energyefficient walking on rough surfaces, compared to other small legged robots (less than $8 \mathrm{~kg}$ ). We will conclude the thesis in Chapter 6 where we will summarize all main findings and provide an outlook for future investigations. 


\section{2 \\ Related Methodologies and Robots}

The related methodologies consist of neural control and compliance control (see Fig. 2.1), which produce coordinated gaits and compliant joint motions for adaptive and energy-efficient legged locomotion. Thereinto, compliance at the end-effectors can be typically implemented in two methods: passive and active compliance. Their advantages and disadvantages in the applications of coordinated and compliant legged robot locomotion are reviewed in the first three sections. In the final section, we describe the motivations of using a bio-inspired legged robot with six three-jointed legs, to study adaptive and energy-efficient locomotion with many DOFs (degrees of freedom). The section is organized as three subsections: why use legged robots, why use six-legged robots, and why use three-jointed legs. Therefore, the six-legged robot AMOS is used to validate adaptive and energy-efficient walking under our proposed method (see Fig. 1.5). This method virtualizes the agonist-antagonist mechanisms to reduce control and structural complexities that intrinsically result from passive compliance control (see the red texts in Fig. 2.1). Moreover, the virtual agonistantagonist mechanisms, neural control, and sensorimotor learning act together to achieve variable compliant and stable locomotion under active compliance control, which inherently gives rise to locomotor instabilities on rough terrain (e.g., gravel). Note that since this chapter only focuses on the classical and our developed controllers on physical robot control, the theoretical neuromechanical (i.e., anchor) and muscle models are discussed in Chapter 1.1 and Chapter 3.

\subsection{Neural control}

Neural control focuses often on modeling the nervous system for coordinated and adaptive legged locomotion over different surfaces (Ijspeert, 2008; Manoonpong et al., 2007, 2013b; Steingrube et al., 2010). In a model of modular neural control on insect locomotion (Büschges, 2005), for example, the basic rhythmic outputs are produced by a central pattern generator $(\mathrm{CPG})$ where there are two neurons that interact with each other. Therefore, a two-neuron CPG (see Fig. 2.2 (a)) is applied to produce the basic rhythmic outputs in neuromechanical control of the insect-like robot AMOS. 


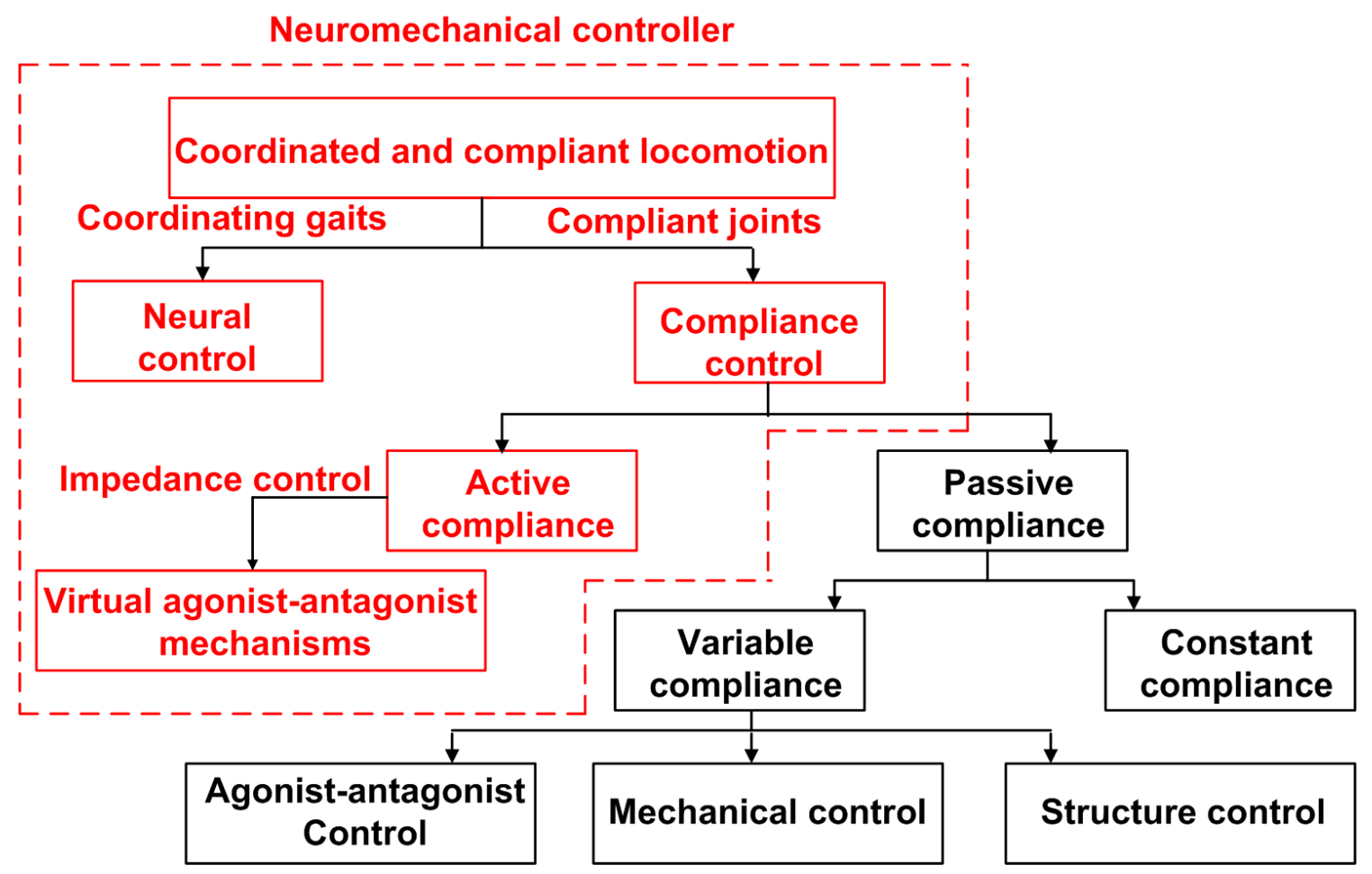

Figure 2.1: Taxonomy of the methodologies for achieving coordinated and compliant locomotion. The major methods are neural control and compliance control, which produce coordinated gaits and compliant joint motions for adaptive and energyefficient legged locomotion. By virtualizing the agonist-antagonist mechanisms, our proposed method (see the red texts) reduces control and structural complexities that intrinsically result from passive compliance control. Moreover, it utilizes a proximodistal gradient and sensorimotor learning to achieve stable and adaptive compliance locomotion under active compliance control, which intrinsically results in unstable locomotion.

One can see that only changing its modulatory input $S$ results in the basic rhythmic outputs with different frequencies (see Fig. 2.2 (b)) that enable AMOS to produce the nine insect-like gaits (see Figs. 5.1). Whereas more parameters of other CPGs need to be tuned to generate less insect-like gaits of the hexapod robots (Yu et al., 2011; Campos et al., 2010) (see Table 2.1).

Mere neural control, however, may lead to energy-inefficient legged locomotion on different surfaces (Xiong et al., 2014a, 2013), since legged robots cannot achieve variable compliant joint motions. For instance, the cost of transport (COT) of an insect-like robot (i.e., Gregor I) during walking over an uneven surface (i.e., obstacle course) is 70 when only a cellular neural network is used as its CPG (Arena et al., 2006). Combining CPGs with forward models can improve the energy efficiency 
Table 2.1: The CPGs applied to the hexapod robots

\begin{tabular}{|l|c|c|c|}
\hline Oscillators & CPGs & Gaits & Amounts of tuned parameter(s) \\
\hline $\mathrm{SO}(2)$ & $\mathbf{1}$ & $\mathbf{9}$ & $\mathbf{1}$ \\
\hline Hopf & 6 & 3 & 6 (Campos et al., 2010) \\
\hline Van der Pol & 6 & 3 & 6 (Yu et al., 2013) \\
\hline
\end{tabular}

(a)

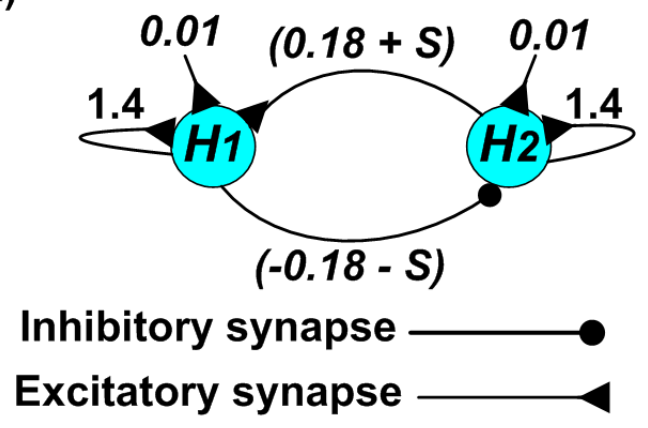

(b) Low frequency $(S=0.04) \quad$ High frequency $(S=0.10)$.

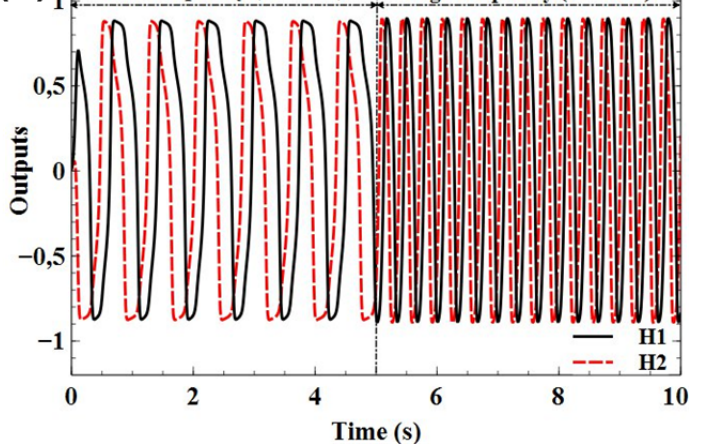

Figure 2.2: A central pattern generator (CPG) with two neurons. The CPG is realized using the discrete-time dynamics of a SO(2) network (Pasemann et al., 2003). It is one part of the modular neural network (Manoonpong et al., 2008) (see Fig. 4.2 (a)) in the neuromechanical controller. (a) The CPG with two full-connected neurons. Changing the modulatory input $S$ produces various basic rhythmic outputs (e.g., low and high frequencies) (Xiong et al., 2012). (b) The examples of basic rhythmic outputs.

of the insect-like (i.e., hexapedal) robots. For example, a CPG combined with an adaptive forward model enables our insect-like robot (i.e., AMOS) to walk over an uneven surface (i.e., coarse gravel) with the COT of 24.5 (Manoonpong et al., 2013b). Adaptive leg motions generated by the neural controllers of the two mentioned robots depend only on the changes of neural outputs of the controllers for walking over uneven surfaces. However, owing to energy-efficiency (Spence, 2011), there are no changes detected in neural activities for controlling key muscles or performing precise limb coordination of insects when walking over an uneven surface (Sponberg and Full, 2008). This finding shows that insects perform adaptive leg motions and compensate perturbations relying more on their biomechanical mechanisms (e.g., muscles), which leads to energy efficiency. Thus our proposed method (see Fig. 1.5) only tunes the biomechanical properties (e.g., stiffness parameters) of the muscle-like mechanisms with a specific set of neural activations when the insect-like robot AMOS walks on a surface. 


\subsection{Passive compliance control}

Passive compliance control is typically regarded as the integration of actuators and viscoelastic mechanics (Ham et al., 2009). In such a case, an actuator may be connected to an elastic element (e.g., a spring), which drives the joint in response to applied loads. Passive compliance control can be divided into constant and variable compliance control (see its taxonomy in Fig. 2.1). In constant compliance control, the compliance of the actuators is fixed and determined by selecting the springs (Pratt and Williamson, 1995). Thus, this character limits their applications to variable compliant joint motions that lead to energy-efficient locomotion over various surfaces. Whereas passive variable compliance theoretically fits to the applications of variable compliant legged robots (Vanderborght et al., 2013), which are generally implemented by structure control (Hollander et al., 2005; Galloway et al., 2011) (see Fig. 2.3 (a)), mechanical control (Vanderborght et al., 2011; Enoch et al., 2012) (see Fig. 2.3 (b)), and agonist-antagonist control (Oku et al., 2012; Vanderborght et al., 2008) (see Fig. 2.3 (c)).

By controlling the length of each leg (see Fig. 2.3 (a)), the hexapod robot EduBot achieves variable compliant leg behaviors for energy-efficient locomotion (Galloway et al., 2011). Its leg compliance, however, must be manually tuned to accommodate its running speeds based on energetic cost. Based on mechanical control (see Fig. 2.3 (b)), the mechanical properties (e.g., dampers) also need to be manually controlled to achieve variable compliant joint motions (Enoch et al., 2012). Moreover, this method requires additional motors to tune the stiffness and damping of such joint motions, thereby leading to bulkier and energy-inefficient legged robots. Compared to the two previous methods, the agonist-antagonist control is the most bio-inspired method, in which two artificial muscles act as like a pair of biological agonist and antagonist muscles to control variable compliant joint motions. This bio-inspired setup leads to faster joint stability (Shadmehr and Wise, 2005), more energy efficiency (Farahat and Herr, 2010) and easier emulations of muscle-like functions (Xiong et al., 2014b). However, it is very difficult to associate such non-linear muscle control with proper joint compliance (Galloway, 2010), which leads to unnatural and unstable locomotion (Rosendo et al., 2013; Tsujita and Miki, 2011; Tsujita et al., 2008). Moreover, the agonist-antagonist designs produce considerable internal forces that require stronger and generally bulkier supporting structures (see Figs. 2.4). Such designs limit their applications to small legged robots with many DOFs (weight less than $8 \mathrm{~kg}$ (Galloway, 2010)). To overcome these drawbacks, our proposed method virtualizes the agonistantagonist mechanisms used to drive the joints (see Fig. 2.3 (d)). The compliance of the joint motions can be adjusted changing the stiffness and damper parameters of the virtual agonist-antagonist mechanisms (Xiong et al., 2014b,a).

In addition, there are intelligent mechanical structures developed for reducing mechanical complexities of legged robots. For example, a four-legged dynamic walking 

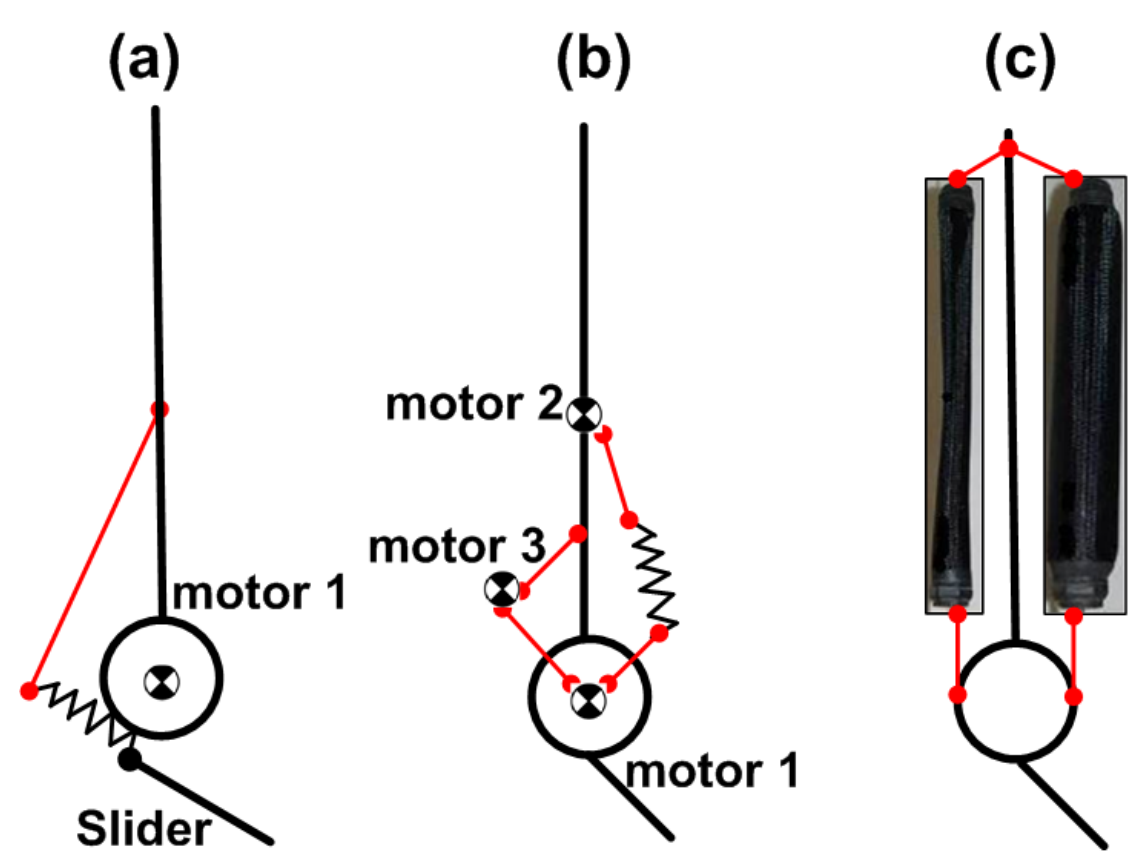

(d)

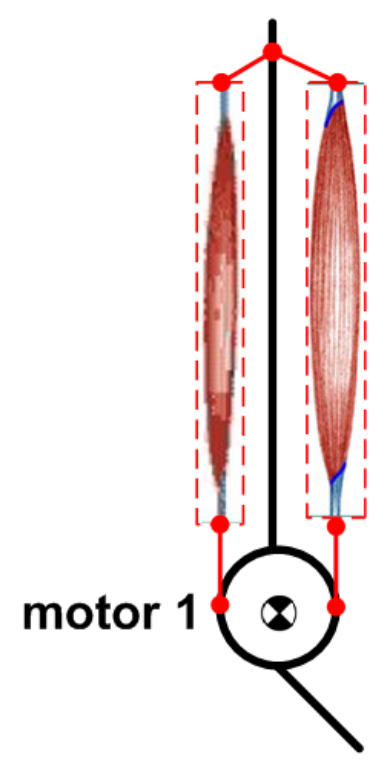

Figure 2.3: Schematic diagrams of passive variable control and our proposed control. They are applied to variable compliance control of a single joint. (a) Structure control. Motor 1 adjusts the length of a compliant element (e.g., a spring or slider) to achieve variable compliant joint motions (Hollander et al., 2005; Galloway et al., 2011). (b) Mechanical control. The control is the mechanically adjustable compliance and controllable equilibrium position actuator (MACCEPA (Vanderborght et al., 2011)) with variable damping (Enoch et al., 2012). Motor 1 and motor 2 are used to control the equilibrium position and tune the joint stiffness, respectively. The joint damping is adjusted by motor 3. (c) Agonist-antagonist control. The variable compliant joint is physically driven by a pair of artificial muscles (e.g., pneumatic artificial muscles (Oku et al., 2012; Vanderborght et al., 2008)). (d) Virtual agonist-antagonist mechanisms (VAAMs). The joint physically driven by a standard servo motor, produces variable compliant motions as if it were driven by a pair of virtual agonist and antagonist muscles (Xiong et al., 2014a). The compliance of the joint motions is determined by the stiffness and damper parameters of the VAAMs (Xiong et al., 2014b).

robot (i.e., Cornell Ranger) achieved more energy-efficient and long-distance walking due to its intelligent mechanical structures (e.g., a light weight, and low-inertia leg design) (Bhounsule et al., 2014). Its mobility, however, is still limited to flat surface walking. Other intelligent mechanical structures are especially developed for small legged robots (Kohut et al., 2011; Baisch et al., 2010; Birkmeyer et al., 2009), but these robots consume much more energy (see Figs. 5.13) due to the limitations of their actuators and structures. For example, the COT of a four-legged robot (Kohut 

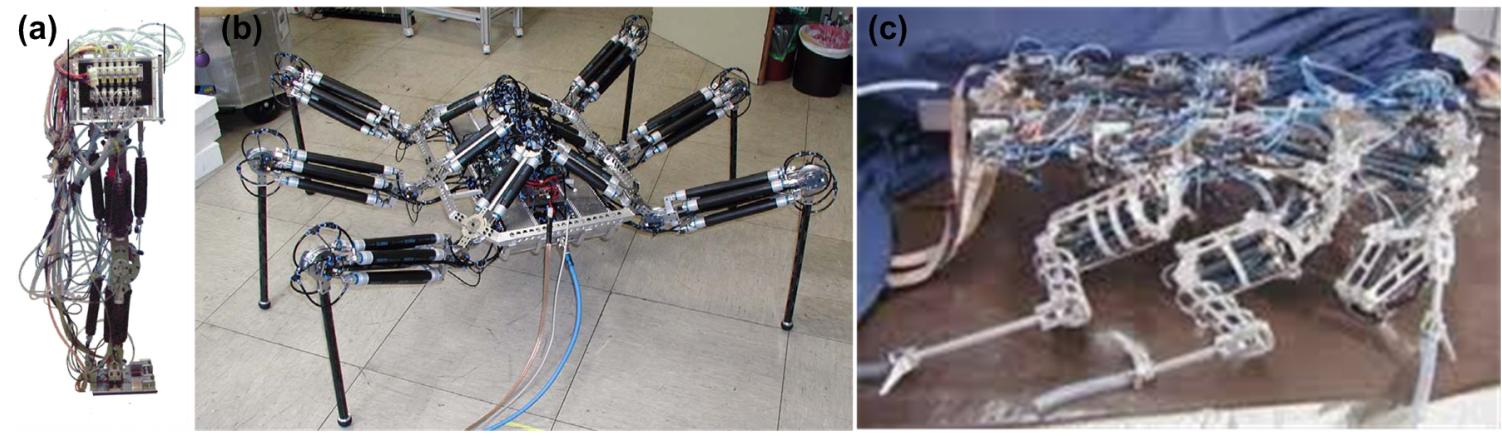

Figure 2.4: Bulky legged robots with antagonistic muscles. (a) A one-legged jumping robot (Iijima et al., 2013) (12.34 kg weight). (b) The six-legged robot AirBug (27.2 kg weight) (Dillmann et al., 2005). (c ) The six-legged robot Ajax (Kingsley et al., 2006) (15.1 kg weight).

et al., 2011) is more than 12,000 when its legs are driven by four shape memory alloy (SMA) actuators. This is because SMA actuators are energy inefficient because of their slow cycle speed.

\subsection{Active compliance control}

Regardless of additional passive components, active compliance control, also known as force control, is mainly achieved by software control of joint torques (Boaventura et al., 2013). Generally, active compliance control can be achieved by impedance control (Hogan and Buerger, 2004; Arevalo and Garcia, 2012; Hwangbo et al., 2014), virtual model control (Pratt et al., 2001; Winkler et al., 2014), and operational space control (Sentis et al., 2010; Hutter et al., 2012). For example, impedance control of a single joint can be modeled as a proportional-derivative (PD) controller (Hwangbo et al., 2014). The output torque $\tau_{o}$ is written as:

$$
\tau_{o}=k_{p}\left(x_{d}-x\right)+k_{d}\left(\dot{x_{d}}-\dot{x}\right),
$$

where $k_{p}$ and $k_{d}$ denote its stiffness and damper (i.e., proportional and differential) parameters, $x_{d}$ and $x$ represent desired joint angle and joint angle feedback, and $\dot{x_{d}}$ and $\dot{x}$ denote their desired velocity and velocity feedback.

Based on angle and velocity feedback, the stiffness (i.e., $k_{p}$ ) and damper (i.e., $k_{d}$ ) parameters of the controller can be tuned in the software programme, which leads to variable compliant joint motions. Thus, active compliance control increases controllability and versatility to legged robots on different environments, compared to passive constant compliance (Pratt and Williamson, 1995). Moreover, one of its advantages over passive variable compliance is reducing control efforts and design complexities of legged robots (Vanderborght et al., 2013; Ham et al., 2009). This advantage leads to 


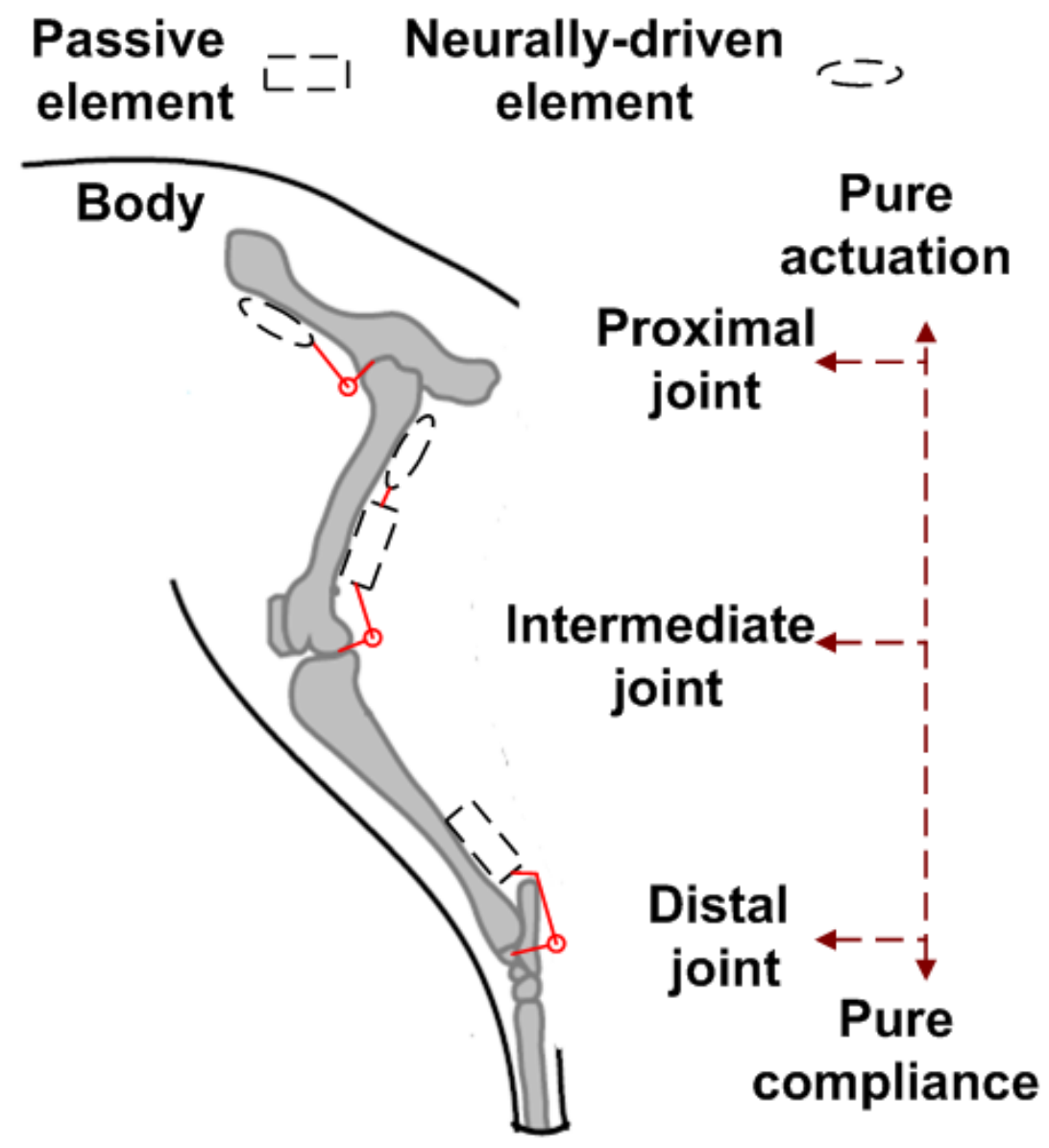

Figure 2.5: A proximo-distal gradient applied to legged locomotion. In such a gradient, neural control and muscle functions vary from the proximal joint to the distal joint in the leg (see the texts for details). Such a gradient had also been found in legged animal locomotion (Lee et al., 2008a; Nishikawa et al., 2007; Daley and Biewener, 2011, 2006). Note that the schematic diagram of the three-jointed leg is modified from Arnold et al. (2013).

its easier applications to small legged robots with many DOFs (weight less than $8 \mathrm{~kg}$ (Galloway, 2010)). Based on active compliance control, the variable joint motions are implemented on our hexapod robot AMOS with 19 DOFs. The proposed neuromechanical control coupled with sensorimotor learning provides three novel ways to solve two inherent problems and one unsolved problem of legged locomotion under active compliance control: 1) use insect-like gaits to improve locomotor energy efficiency on different surfaces, 2) use a proximo-distal gradient of neural control and muscle functions to enhance locomotor stability, 3) use sensorimotor learning to online learn the stiffness parameters of the muscle-like mechanisms coordinating many DOFs. Firstly, a bio-inspired neural network of the proposed neuromechanical controller is 


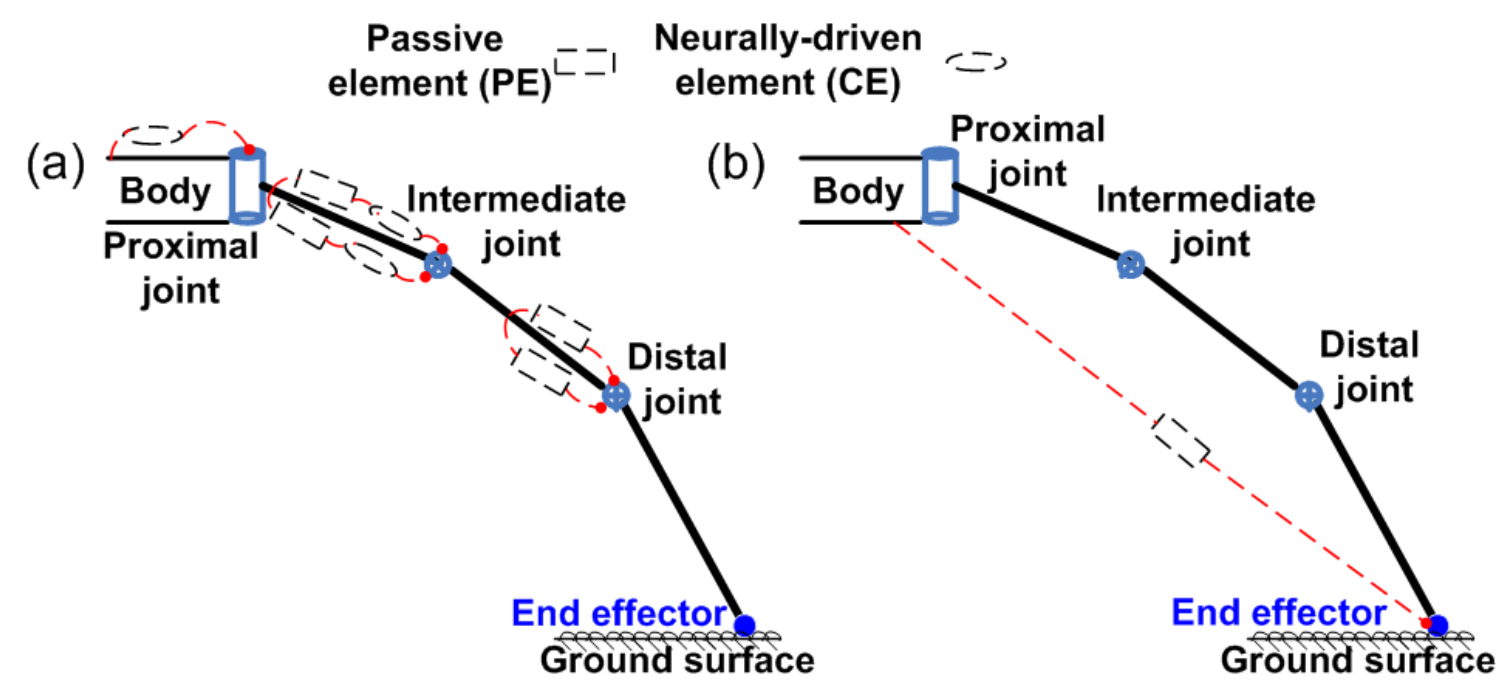

Figure 2.6: Controllers that use the virtual elements. (a) The VAAM controller that uses the virtual contractile and passive elements (i.e., CEs and PEs, see Figs. 3.4). The controller is based on the proximo-distal gradient (see Fig. 2.5). (b) A virtual model controller (Pratt et al., 2001; Hutter et al., 2011) that only exploits a virtual passive element (e.g., spring) attaching the body to the end effector. See the comparisons between the two controllers in Table 2.2.

used to produce coordinated insect-like gaits for achieving energy-efficient locomotion on different surfaces (Nishi, 1998; Nishii, 2000). Secondly, a versatile legged robot should achieve variable compliant joint motions that accommodate different gaits and surfaces, which leads to energy-efficient locomotion. However, under classical active compliance control, transient responses of such compliant motions intrinsically result in unstable locomotion on rough surfaces (Fukuoka et al., 2003; Garcia and de Santos, 2006). To solve this inherent problem, a proximo-distal gradient of neural control and muscle functions (see Fig. 2.5) is applied to the neuromechanical controller to enhance locomotor stability. In such a gradient, the proximal joints are under feed-forward neural control and less sensitive to the loads. Thus, the proximal joints mainly act as the actuation that produces energy. By contrast, the distal joints are more sensitive to the loads, and under feedback (e.g., force) control that allows variable compliant joint motions against the loads. Therefore, the distal joints greatly serve as the compliance that absorbs energy. This proximo-distal gradient enhances locomotor stability of legged animals on rough terrain (Lee et al., 2008a; Nishikawa et al., 2007; Daley and Biewener, 2011, 2006). Based on the gradient, the passive elements (i.e., PEs) of a virtual agonist-antagonist mechanism (VAAM, see Figs. 3.4) act as the compliant mechanisms driving a distal joint of a legged robot while its proximal joint can be actuated by the contractile elements (CEs) of a VAAM. Such passive compliance and active actuation make the VAAM differ from virtual model 
Table 2.2: The comparisons between the VAAM control and VMC

\begin{tabular}{|l|c|c|c|}
\hline Controllers & $\begin{array}{c}\text { Locomotor } \\
\text { stability (see } \\
\text { Fig. 2.7) }\end{array}$ & $\begin{array}{c}\text { Muscle-like } \\
\text { functions }\end{array}$ & Sensory feedback \\
\hline VAAM & enhanced & $\begin{array}{c}\text { springs } \\
\text { and brakes } \\
\text { (see } \\
\text { Figs. } 3.17 \\
\text { and } 3.10 \\
(b))\end{array}$ & $\begin{array}{c}\text { at the end effector } \\
\text { of each leg } \\
\text { only force sensing }\end{array}$ \\
\hline VMC & reduced & not shown & $\begin{array}{c}\text { force/torque feedback } \\
\text { at each joint }\end{array}$ \\
\hline
\end{tabular}

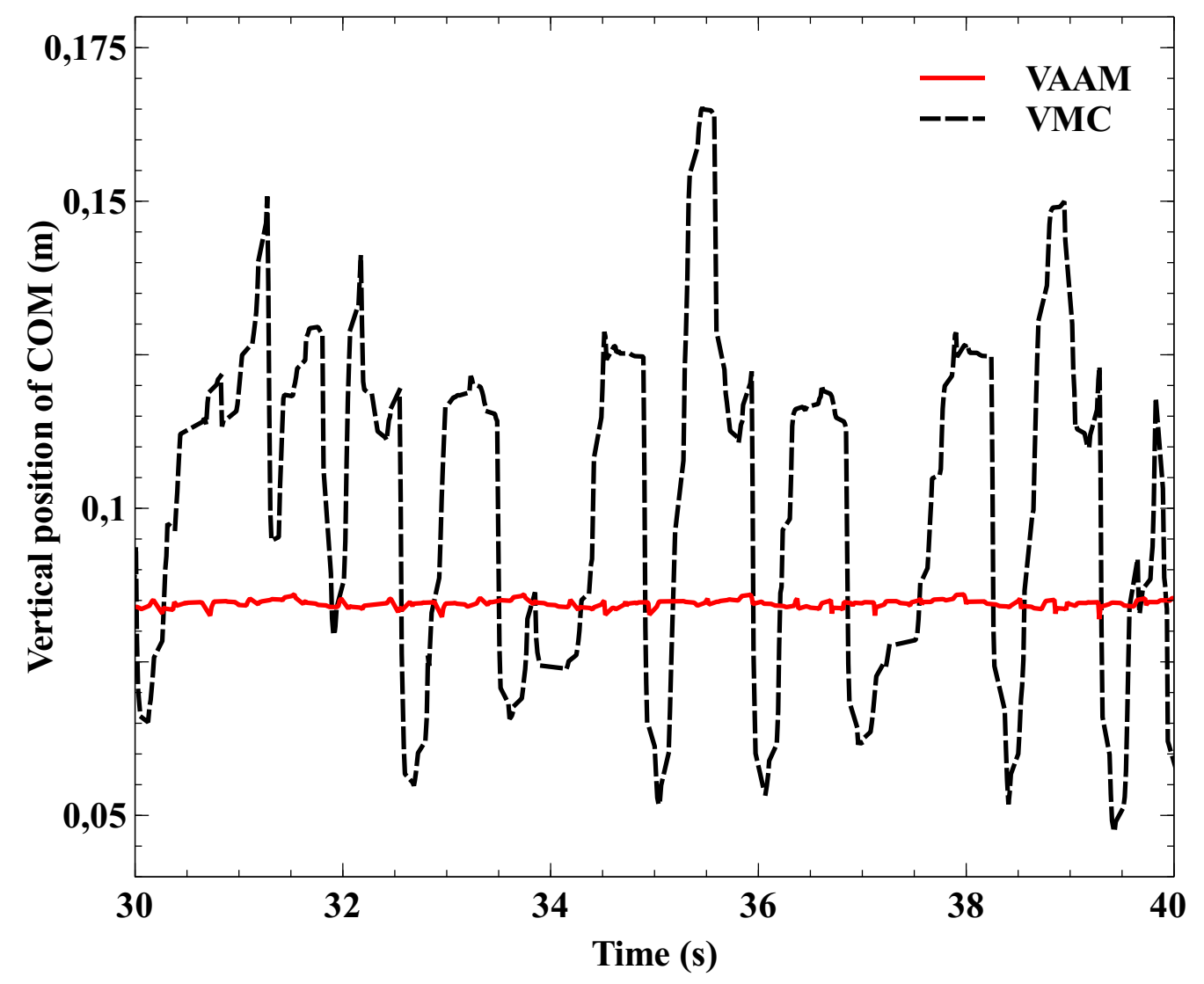

Figure 2.7: Vertical positions of COM (Center Of Mass) of the hexapod robot AMOS. The experiments are conducted in the physical simulator lpzrobots (Der and Martius, 2012). The VAAM control enables AMOS to walk stably (smaller body oscillation), compared to virtual model control (VMC) (see VAAM and VMC control in Figs. 2.6). 
control (VMC), which only contains a virtual passive element (e.g., spring) attached to the robot as if it had exited (Pratt, 2000; Pratt et al., 2001) (see Figs. 2.6). In contrast to VMC controllers (Hutter et al., 2011; Ajallooeian et al., 2013), the VAAM not only includes the virtual passive elements (PEs) to act as muscle-like mechanisms, but also integrates the virtual contractile elements (CEs) to serve as neural control at the joints of legged robots. Furthermore, such elements of the VAAM allow the joints of legged robots to act as different roles (i.e., compliance or actuation, see Fig. 2.5) that enhance locomotor stability under active compliance control(Xiong et al., 2014a) (see Table 2.2). The VAAM control is more bio-inspired control by integrating neural control and muscle-like functions, compared to VMC that only utilizes the virtual passive element to produce compliant leg behaviors. As a result, the VAAM control enables AMOS to not only achieve more stable walking under active compliance control (see Fig. 2.7), but also easily emulate muscle-like functions (e.g., brakes, see Figs. 3.11 (c) and (d)). Thirdly, adaptive compliance control on a physical legged robot with many DOFs remains an important and unsolved problem in a context of energy-efficient walking on different surfaces (Arevalo and Garcia, 2012; Nishikawa et al., 2007). Specifically, self-adjusting its parameters (i.e., $k_{p}$ and $k_{d}$ in Eq.(2.1)) is a difficult problem in a context of controlling energy-efficient legged locomotion with many DOFs.

\subsection{Legged robots}

Adjusting leg compliance is one of the most important hallmarks of energy-efficient legged locomotion over tough surfaces (e.g., soft and elastic sponges) (Spence et al., 2010; Spence, 2011; Farley et al., 1998; Ferris et al., 1998). Inspired by this, adjustable compliance leg behaviors enable legged robots to achieve more efficient and adaptive locomotion on different surfaces (Xiong et al., 2014a; Galloway et al., 2013; Semini et al., 2011). In addition, locomotor efficiency and adaptivity of legged robots are determined by the numbers of their legs and joints that depend on the usages (e.g., stable locomotion).

\subsubsection{Why use legged robots}

Legged vehicles (e.g., robots) are capable of traversing over most landmasses on the Earth, yet less than half of them is accessible to wheeled or tracked vehicles. Thus, the motivation of using and studying legged robots stem from their mobility and adaptivity over rough terrains (see Figs. 2.8), compared to wheeled robots.

There are many advantages of legged robots over wheeled robots:

- legged robots can well negotiate discontinuous terrains (e.g., see Fig. 2.8 (a)) (Manoonpong et al., 2014), which are unattainable for most wheeled robots to get across; 
(a)
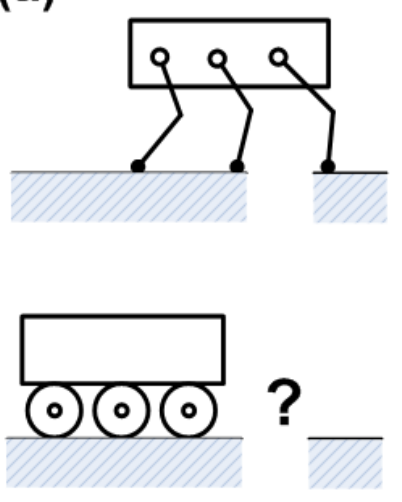

(b)
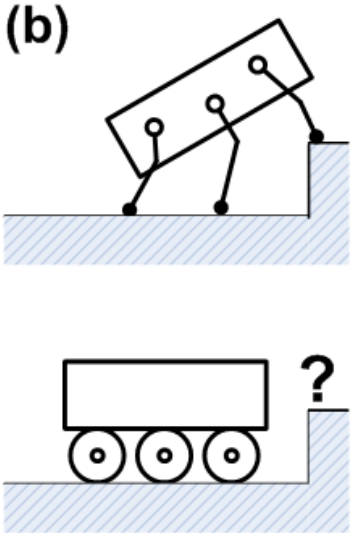

(c)

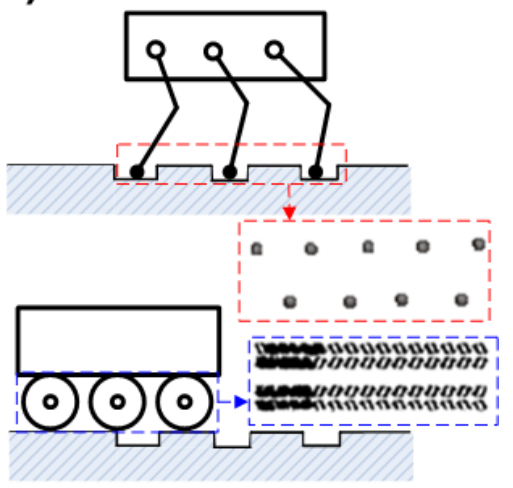

Figure 2.8: Legged robots versus wheeled robots on rough terrains. (a) Discontinuous terrain. (b) Obstacles. (c) Irregular terrain. Legged robots have better mobility over rough terrains, compared to wheeled robots. In addition, legged robots suffer less interferences with the irregular terrain (see the paths of the footholds within the red and blue dashed rectangles), especially soft or gravel terrain. Such less interferences mean lower risks of slippages and being stuck, and lower resistances.

- legged robots can climb over large obstacles (e.g., see Fig. 2.8 (b)) (Goldschmidt et al., 2014), which are inaccessible for most wheeled robots;

- legged robots suffer less interferences with the irregular terrains (e.g., see Fig. 2.8 $\left.(\mathrm{c})^{1}\right)$, compared to the wheeled robots. This leads to lower resistances and risks of environmental damages.

This is because wheels only roll, which decreases the mobility and adaptivity of such wheeled robots. Whereas legged robots can perform various locomotor patterns (i.e., gaits) to adapt different surfaces. Although legged robots have many advantages over wheeled robots on rough terrains, adding legs to robots and controlling them in an efficient and adaptive way is a challenging task. This is because even humans take many efforts and years to learn to walk, not to mention designing legs for the robots and teaching them to walk in such a way. Specifically, legged robots need to learn and adapt to different environments using different walking patterns (i.e., gaits) and variable compliant joint motions, which is a major goal of our presented work in this thesis. 

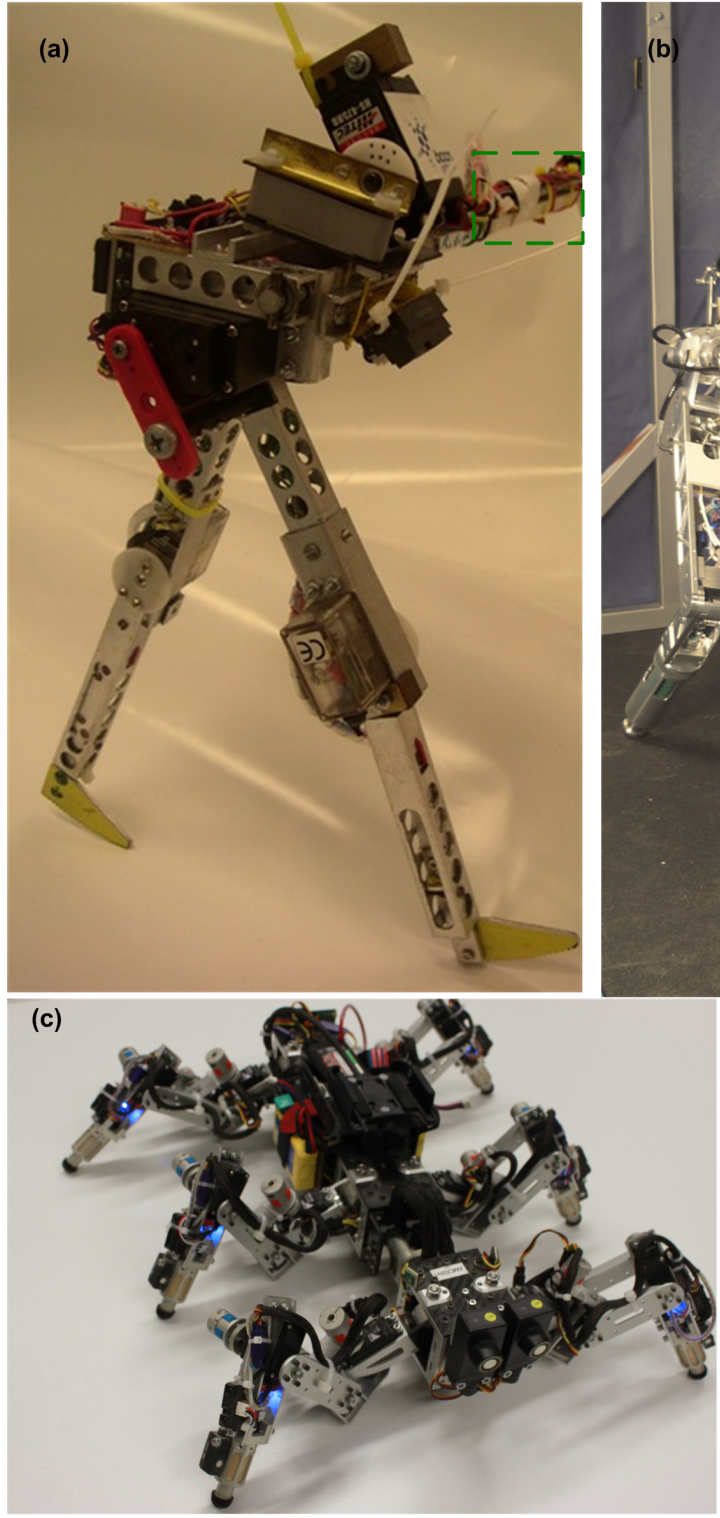

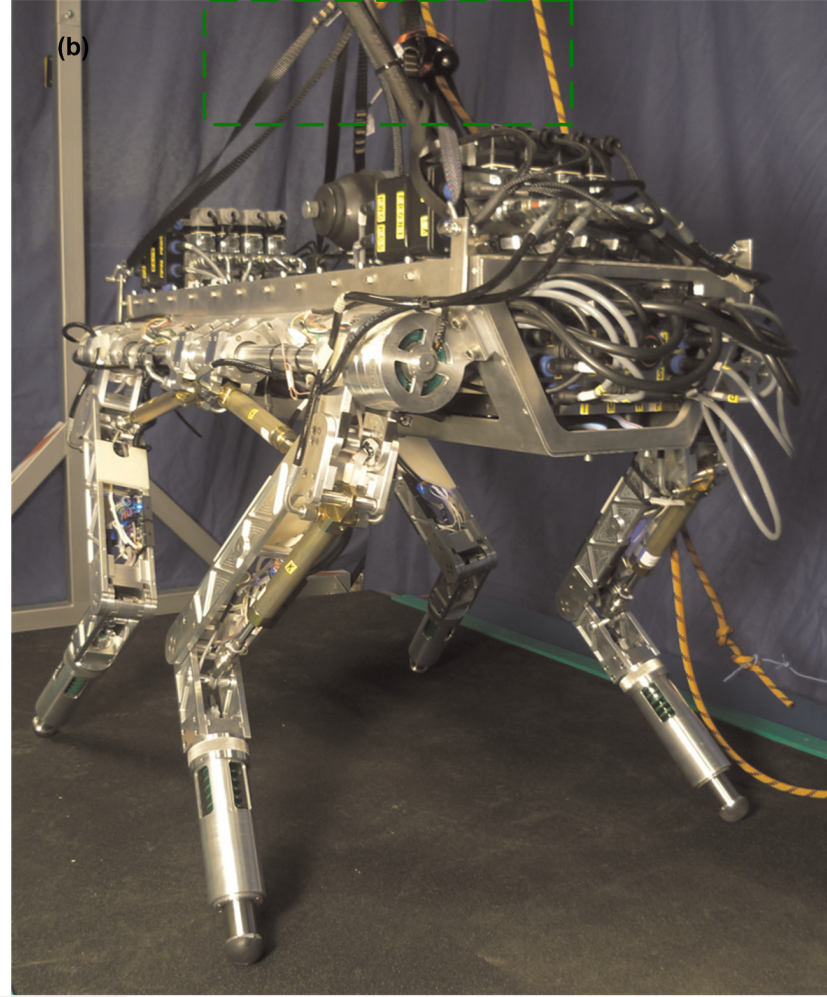

(d)

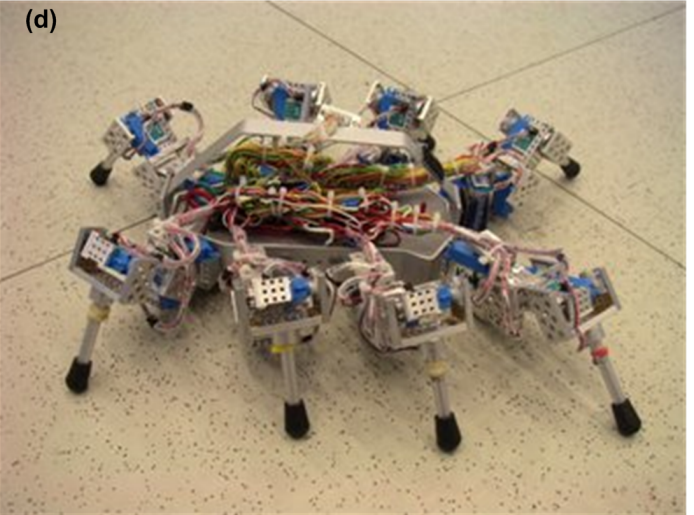

Figure 2.9: Multi-legged robots. (a) The two-legged robot RunBot (0.47 kg weight, four degrees of freedom (DOFs)) (Manoonpong and Wörgötter, 2009). (b) The fourlegged robot HyQ (90 kg weight, 16 DOFs) (Semini et al., 2011). (c) The six-legged robot AMOS (Advanced MObility Sensor driven-walking device, $5.4 \mathrm{~kg}$ weight, 19 DOFs) (Manoonpong et al., 2013b). (d) An eight-legged robot (2.7 kg weight, 24 DOFs) (Manoonpong et al., 2008). With increased dynamics (i.e., less legs and joints), two-legged and four-legged robots usually require additional supporters (see green-dashed rectangles). Whereas the six-legged and eight-legged robots perform more stable locomotion, but require to coordinate inter- and intra-legs. 


\subsubsection{Why use six-legged robots}

In addition to mobility, stability is very essential to legged systems using various gaits, which emerge from compliant legs (Ernst et al., 2012; Seyfarth et al., 2006). Stable legged systems (e.g., robots) can be divided into two different groups: dynamically and statically stable systems (Tomaž, 2004; Full et al., 2002). Belonging to the first group, the two-legged and four-legged robots (see Figs. 2.9 (a) and (b)) simultaneously use less than three legs on the ground. Due to less legs, it is more difficult to stabilize them during their locomotion. Therefore, most current two-legged and four-legged robots usually require additional supporters (e.g., see the green-dashed rectangles in Figs. 2.9 (a) and (b)) due to their inherent instabilities. Furthermore, such instabilities will cause noisy sensing (e.g., force sensing), which is inappropriate for our presented work where force sensing is essential for the robot to classify surfaces and achieve energy-efficient locomotion. From this point of view, the two-legged and fourlegged robots are too unstable and vulnerable to be applied to rough surfaces (e.g., gravel), compared to six-legged and eight-legged robots. The six-legged and eightlegged robots (see Figs. 2.9 (c) and (d)) often walk in more stable ways where at least three legs are planted on the ground. Moreover, the six-legged robots can still use the rest of the legs to move over such surfaces, even when some of them are damaged (Manoonpong et al., 2013b; Koos et al., 2013; Johnson et al., 2010). This is because six-legged robots can produce stable insect-like gaits where their COMs (centers of mass) are well maintained within the sets of alternating polygons of support. Full argued that this character is an important reason why six-legged systems (e.g., robots) rule (McNichol and Full, 2002). Moreover, Hughes stated that the six-legged systems are the 'end-products of evolution' in terms of statical stability (Hughes, 1952). Due to energy efficiency, a six-legged robot is better for our presented work, compared to an eight-legged robot. This is because driving additional motors consumes more energy under active compliance control.

\subsubsection{Why use three-jointed legs}

For the six-legged (i.e., hexapod) robots, their mobility and flexibility are also determined by the numbers of the joints of the legs. Typically, the numbers of the joints range from one to five, which vary with different applications. For example, the single-jointed and two-jointed legs (see Figs. 2.10 (a) and (b)) are easily controlled (Galloway et al., 2013; Soltero et al., 2013), thereby leading to more mobile and energy-efficient locomotion. Whereas four-jointed and five-jointed legs can perform more flexible tasks (e.g., manipulation) (Rönnau et al., 2014) and well imitate the morphology of a cockroach (Kingsley et al., 2006), respectively. Unlike the above

\footnotetext{
${ }^{1}$ The schematic diagram of the footholds (see Fig. 2.8 (c)) is modified from a slide at http: //leggedrobotics.ethz.ch/lib/exe/fetch.php?media=background_slidesforwebpage_1.pdf
} 
mentioned applications, the presented work in this thesis focuses on achieving flexible leg behaviors and energy-efficient locomotion on rough surfaces (e.g., gravel). Thus, three-jointed legs are chosen as the trade-off between locomotor flexibility and efficiency in the presented work.

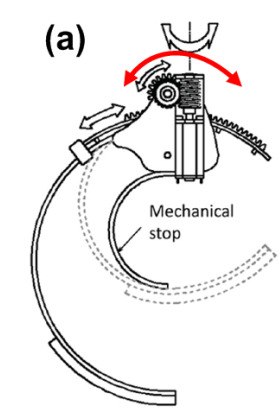

(d)

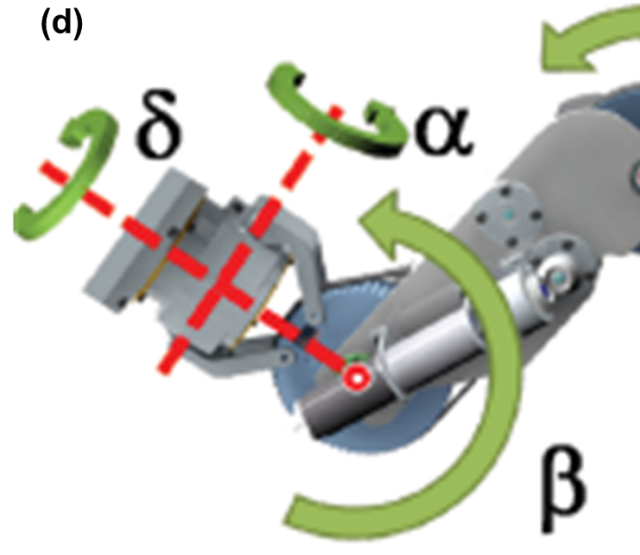

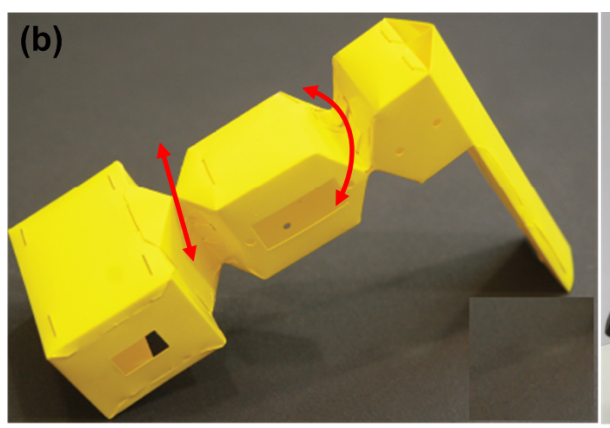

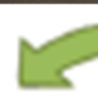

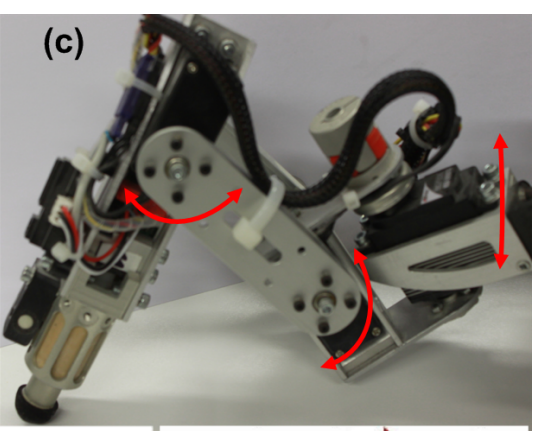

(e)

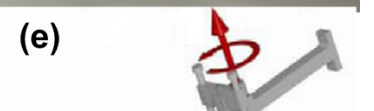

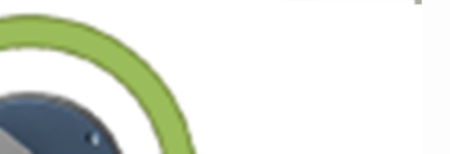
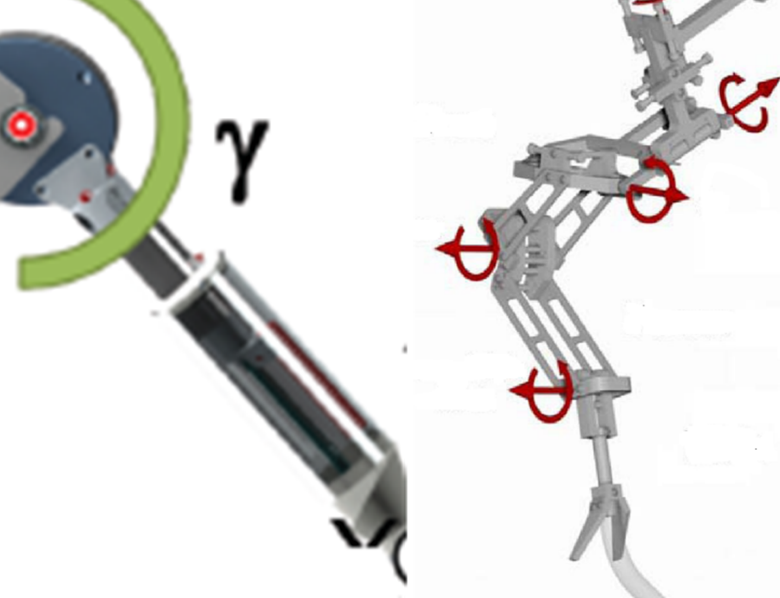

Figure 2.10: Jointed legs of the hexapod robots. Their numbers of the joints range from one to five. (a) A single-jointed leg of EduBot (Galloway et al., 2013). (b) A two-jointed leg of FireAnt (Soltero et al., 2013). (c) A three-jointed leg of AMOS (Manoonpong et al., 2013b). (d) A four-jointed leg of LAURON V (Rönnau et al., 2014). (e) A five-jointed leg of Ajax (Kingsley et al., 2006). The numbers of the joints vary with different applications.

In addition, three-jointed legs are appropriate for legged robots to prevent slippery behaviors on certain surfaces (e.g., muddy grassland or gravel). For example, a threejointed leg produces an additional force to the legged robot in comparison to a twojointed leg. This character is especially essential to prevent slippery conditions where transverse force at ground contact is subject to be decreased. Such preventions had been found by the computer animations of multi-legged locomotion by Raibert and Hodgins (Raibert and Hodgins, 1991). Based on this finding, Nagle used a simulated 
and articulated hopper to explained the advantages of three-jointed legs over other jointed legs (Nagle, 1995b,a). Moreover, three-jointed legs are biologically inspired by the morphology of a cockroach leg (see Figs. 3.3 (b) and (c)), thereby leading to easily emulating insect-like muscle functions and gaits on our insect-like robot AMOS. 


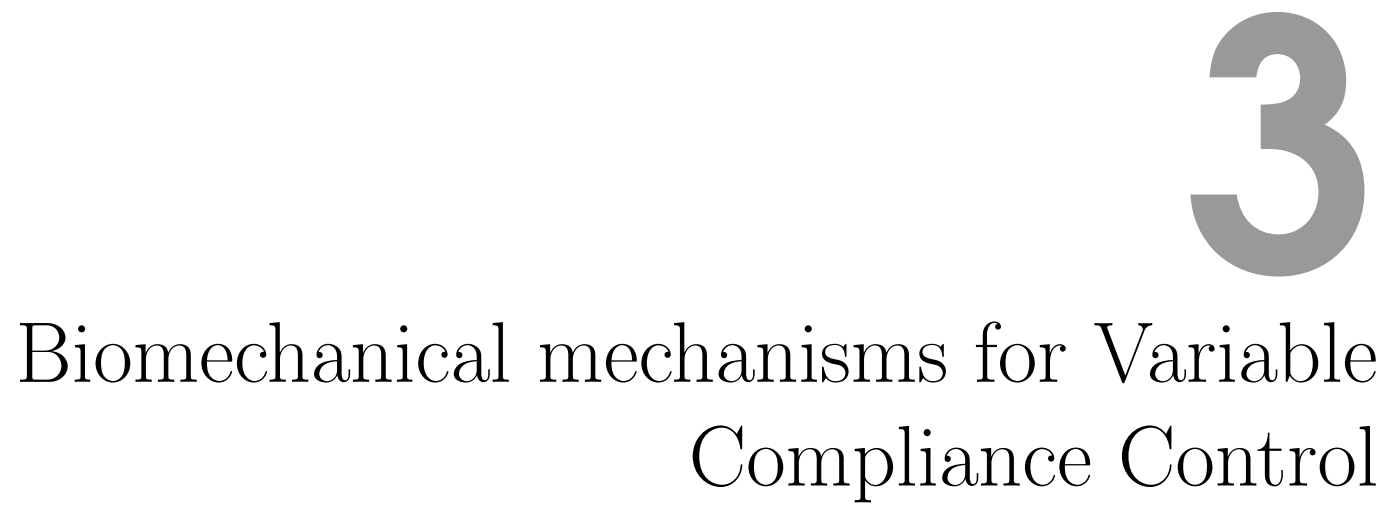

In Chapter 2, one can see that the biomechanical mechanisms such as pneumatic artificial muscles are developed and applied to legged robot locomotion (Oku et al., 2012; Vanderborght et al., 2008). Their aims are to emulate the musculoskeletal systems of legged animals to improve current robotic performances (e.g., energy efficiency). However, such aims and emulations are the paradoxes where closer to those of the animals the musculoskeletal systems of the robots are, poorer the robotic performances (e.g., mobility) are. For example, although the morphologies of the hexapod robots AirBug and Ajax (see Figs. 2.4 (b) and (c)) are closer to those of the insects, their energy efficiency and mobility are degraded (Dillmann et al., 2005; Kingsley et al., 2006). By contrast, the energy efficiency and mobility of another insect-like robot Edubot are greatly upgraded because of a largely simplified musculoskeletal system (Galloway et al., 2013). In Edubot, there are only one DOF (degree of freedom) and a compliant slider in each leg that are different from neurally coordinated many DOFs and several muscles in that of an insect (e.g., Blaberus discoidalis). Therefore, this simplest (i.e., template, see Fig. 1.4 (b)) system failed to model a nervous system, muscles, joints, and legs with many DOFs like in insects (Holmes et al., 2006). Moreover, due to its simplicity, EduBot did not show insect-like muscle functions (e.g., brakes) that are comparable to those of biological insect muscles (Ahn and Full, 2002; Sponberg et al., 2011). EduBot only presented variable compliant legs by using the geared DC motors to manually adjust the lengths of the compliant sliders.

Although biological muscles are usually considered as motors that produce positive work (Nishikawa et al., 2007), they can perform other functions like struts, springs, dampers, and brakes (Dickinson et al., 2000; Gabaldón et al., 2004; Roberts et al., 1997; Fish, 2002). For example, in an insect (i.e., Blaberus discoidalis), its muscle 179 acts as a brake that produces negative work by absorbing the energy from the perturbed loads (Ahn and Full, 2002). This muscle function (i.e., the brake) enables the insects to accomplish joint stability and locomotor mobility over difficult terrains (Dickinson et al., 2000; Sponberg and Full, 2008; Sponberg et al., 2011; Full et al., 2002). The muscle function is quantified using the work loop technique 
(Biewener, 2003; Ahn, 2012) where the muscle mechanical responses (i.e., muscle force and length) are measured. Generally the mechanical responses can be represented by a simplified Hill's muscle model (Ghigliazza and Holmes, 2005; Hill, 1938; Zajac, 1989) where each muscle contains a contractile element $\mathrm{CE}$ and a parallel element PE (see Fig. $3.2(\mathrm{a})$ ). The $\mathrm{CE}$ and $\mathrm{PE}$ produce active force $F^{C}$ and passive force $F^{P}$ that constitute the total muscle force $F^{T}$,

$$
F^{T}=F^{C}+F^{P}
$$

where the active force $F^{C}$ is a product of a neural activation $N^{C}$, a length-dependent function $f(\bar{L})$ (see Fig. $3.1($ a)), and a velocity-dependent function $f(\bar{V})$ (see Fig. 3.1 (b)):

$$
F^{C}=F_{0} N^{C} f(\bar{L}) f(\bar{V}),
$$

where $F_{0}$ denotes the scale factor, $N^{C}$ is the neural activation that follows two linear second ODEs (ordinary differential equations) and a nonlinear algebraic function (see Eqs.(1-3) in (Kukillaya and Holmes, 2009)), the function $f(\bar{L})$ is given by a third polynomial function (see Eq.(4) in (Kukillaya and Holmes, 2009)) of the normalized muscle length $\bar{L}$, and the function $f(\bar{V})$ is represented by a piecewise-smooth function (see Eqs.(6-7) in (Kukillaya and Holmes, 2009)).
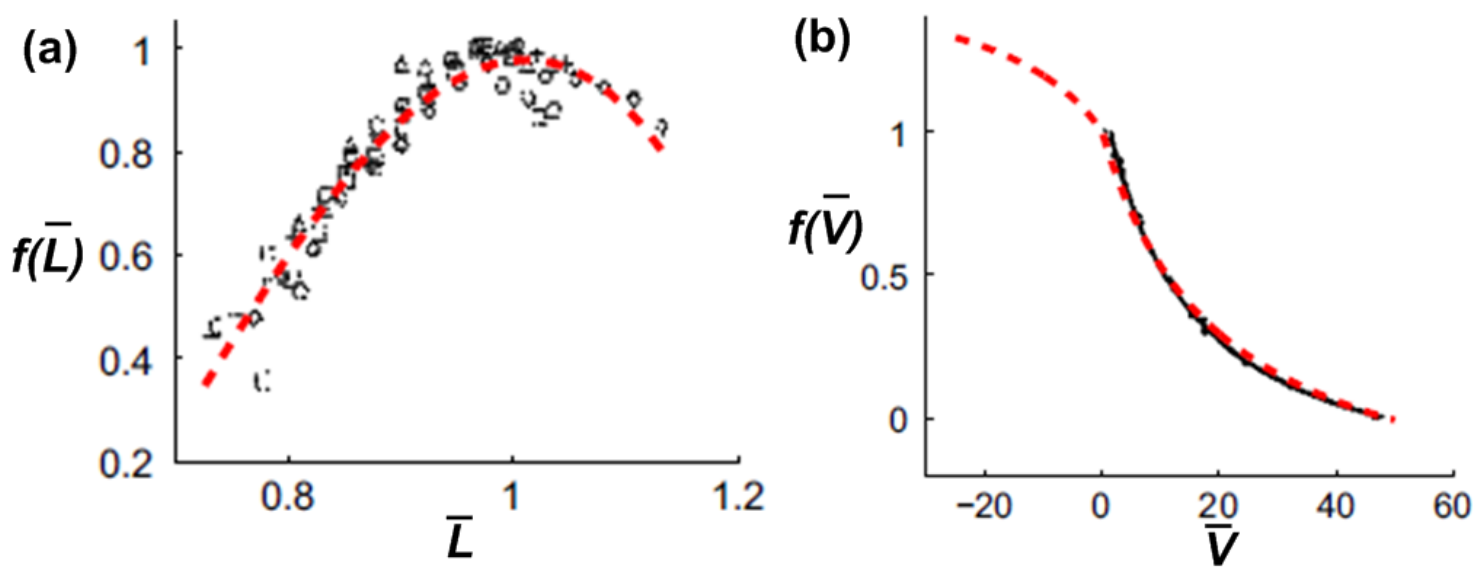

Figure 3.1: The length-dependent and velocity-dependent functions $f(\bar{L})$ and $f(\bar{V})$ of an insect muscle. (a) The length-dependent function $f(\bar{L})$. (b) The velocitydependent function $f(\bar{V})$. The figures are modified from Kukillaya and Holmes (2009).

In addition to the active force $F^{C}$, the passive force $F^{P}$ also contributes the total muscle force $F^{T}$. $F^{P}$ is given by:

$$
F^{P}=K \Delta L+D V
$$


where $K$ and $D$ denote the stiffness and damper parameters of the parallel element $\mathrm{PE}$, and $\Delta L$ and $V$ are its displacement and velocity.

(a)

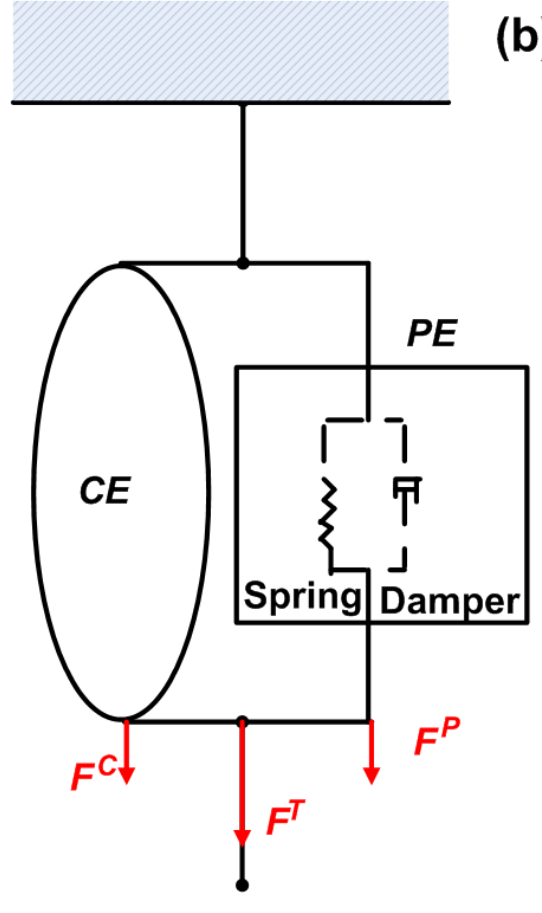

(b)

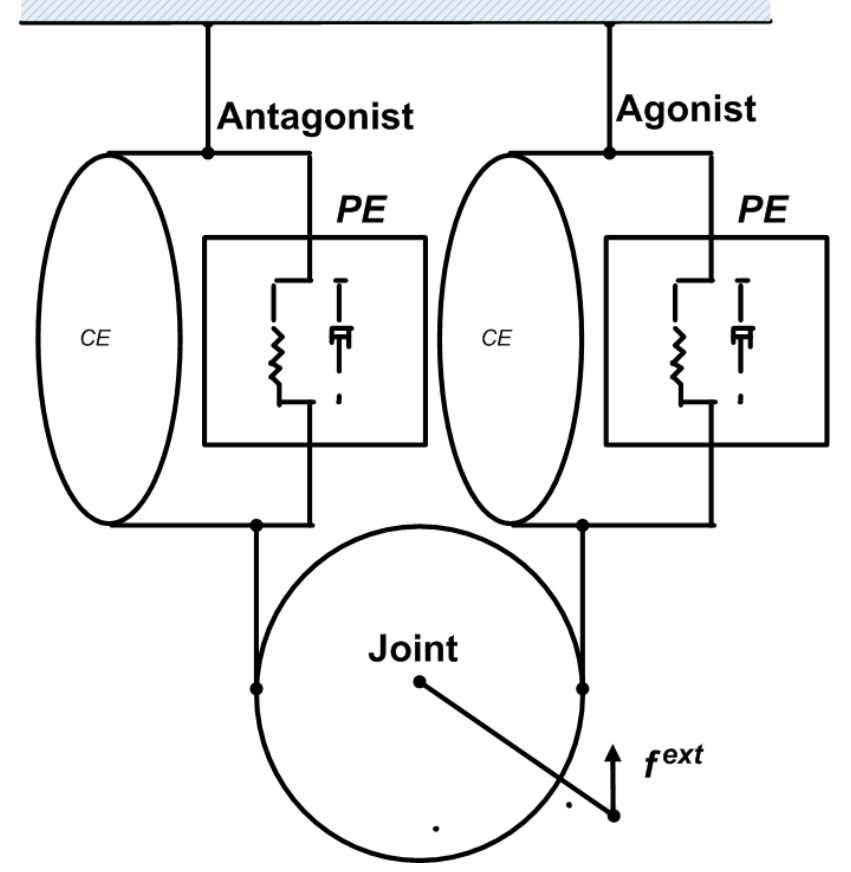

Figure 3.2: Schematic models of the insect muscle. (a) An insect muscle model (Ghigliazza and Holmes, 2005). The model is a simplified Hill muscle model (Hill, 1938; Zajac, 1989). Here each muscle consists of a contractile element and a parallel element (i.e., $C E$ and $P E$ ), which produce contractile force $F^{C}$ and parallel force $F^{P}$, respectively. Its total produced force $F^{T}$ is the sum of $F^{C}$ and $F^{P}$. (b) A pair of the agonist and antagonist mechanisms. Here the insect joint that suffers the external force $f^{e x t}$ is driven a pair of the agonist and antagonist mechanisms. The modeling of each muscle is based on the simplified insect muscle model. More details of the simplified model can be seen at Fig. 3.4.

The insect muscle model had been applied only to the computer simulations of insect locomotion (Proctor and Holmes, 2010; Kukillaya and Holmes, 2009), because its applications require the hundreds of motor neurons and dozens of tuned parameters. For example, 16 parameters (see the table 3 in (Kukillaya and Holmes, 2009)) need to be tuned to fit the neural activation $N^{C}$, the length-dependent function $f(\bar{L})$, and the velocity-dependent function $f(\bar{V})$ (see Eq.(3.2)) to the experimental data obtained from the insects. This leads to the complexities and difficulties of being applied to physical insect-like robots with musculoskeletal systems. In the musculoskeletal systems of the insects, their passive properties play major roles in joint stability and locomotor mobility over tough terrain (Revzen et al., 2013; Dudek and Full, 2006; 
Haeufle et al., 2010; Koditschek et al., 2004), compared the active properties that are governed by the neural activities. For example,there are no changes detected in neural activities for controlling key muscles or performing precise limb coordination of insects when walking over an uneven surface (Sponberg and Full, 2008). This finding shows that insects perform adaptive leg motions and compensate perturbations by relying more on the passive properties of the muscles.

Inspired by the biological principles mentioned above, Chapter 3 will present that the insect-like robot AMOS can generate variable compliant joint motions against various perturbations (e.g., hand pushing). The implementation relies only on the passive elements (i.e., PEs) of the agonist-antagonist mechanisms (see Fig. 3.2 (b)). As a result, such compliant joint motions lead to the reductions of contact forces and soft interactions against the perturbations. Interestingly, the insect-like muscle functions (i.e., brakes) underlying the variable compliant joint motions are comparable to those of biological insect muscles (Dickinson et al., 2000; Full et al., 1998; Ahn and Full, 2002; Sponberg et al., 2011). In principle, the implementation of such joint motions is comparable to proportional-derivative control (PD control) where software control of joint positions can be achieved adjusting its derivative parameter (i.e., damper parameter) (Tomei, 1991; Luca et al., 2005). This makes the implementation differ from bulky passive variable compliant hardware such as variable stiffness actuators (VSAs) (Ham et al., 2009) and artificial muscles (Schmitt et al., 2012). Taken together, the proposed muscle model (see Fig. 3.2 (b)) provides a way forward to emulate muscle-like functions that are comparable to those of biological muscles. The emulation of the muscle-like functions relies only on the PEs of the agonist-antagonist mechanisms (see Fig. 3.2 (b)) that are implemented on the insect-like robot AMOS.

\subsection{The insect-like robot AMOS}

Here we use an insect-like (i.e., hexapedal) robot (i.e., AMOS, $5.4 \mathrm{~kg}$ weight, see Fig. $3.3(\mathrm{a})$ ) as our experimental platform. It has six three-jointed legs, and each leg (see Fig. 3.3 (b)) emulates the morphology of an insect leg (Zill et al., 2004) (see Fig. 3.3(c)). Every leg has a TC (Thoraco Coxal) joint allowing forward and backward motions, a CTr (Coxa Trochanteral) joint allowing elevation and depression motions, and an FTi (Femur Tibia) joint allowing extension and flexion motions (see Fig. 3.3 (b)). Each joint is physically driven by a standard servo motor (i.e., HSR-5990TG). There is a force sensor (i.e., FS Series Force Sensor) used for detecting an analog force signal at each leg (see $f c_{1-6}$ in Fig. 3.3 (a)). A current sensor, installed inside the body of the hexapod robot, is used to measure the electrical current supplied to all motors of the robot. Here, the current sensor signal is used to calculate power consumption during walking. The sensory data are transmitted via an RS232 serial connection to an external PC on which the controller is implemented. Through the same connection, the final motor commands of the controller are sent to the robot 
joints, each of which is driven by a pair of the virtual muscle-like components. More details of AMOS hardware ${ }^{1}$ can be seen at Manoonpong et al. (2013b).

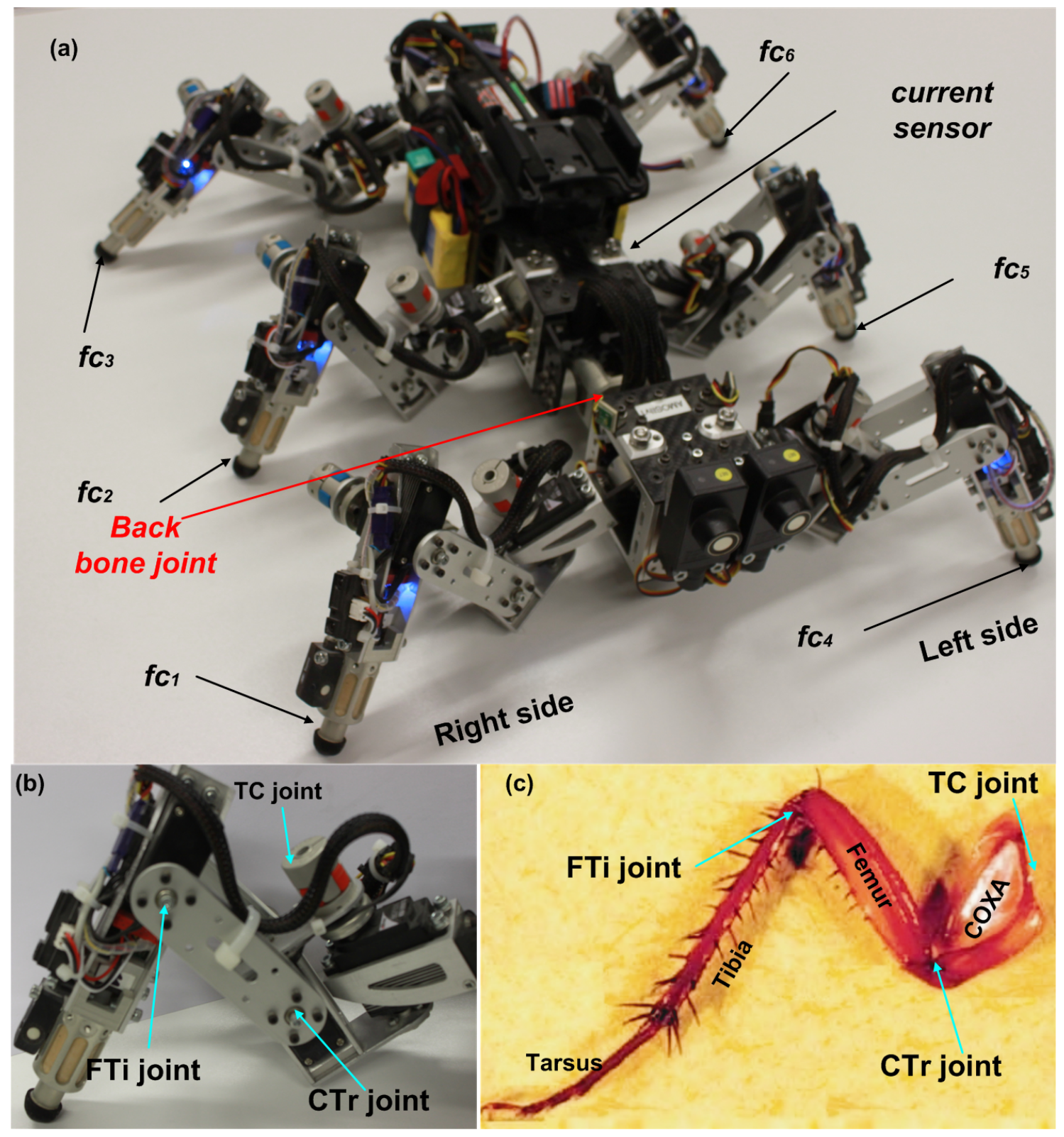

Figure 3.3: An insect-like (i.e., hexapedal) robot AMOS. (a) AMOS and its sensors. $f c_{(1-6)}$ are force sensors. (b) A leg with three degrees of freedom. Note that the tarsus segments are ignored here. (c) An insect leg (modified from Zill et al. (2004)).

${ }^{1}$ http: //manoonpong.com/AMOSII .html 


\subsection{Virtual muscle-like mechanisms for variable com- pliance control}

In our implementations, each virtual muscle-like mechanism is modeled as contractile and parallel elements (see Fig. 3.2 (a)) that produce active and passive forces, respectively. In the case of controlling a physical joint, the joint is driven by two virtual muscle-like components $M 1$ and $M 2$ (see Fig. 3.4(a)) that act together as a virtual agonist-antagonist mechanism (VAAM). Virtual means that the joint, physically driven by a standard servo motor, generates variable compliant motions as if it were driven by a pair of physical agonist and antagonist muscles. The VAAM produces active and passive forces using its contractile and parallel elements (CEs and PEs, see Fig. 3.4(b)). The joint actuation relies on the CEs, while the PEs govern joint compliance.

The parallel elements (i.e., PEs) are modeled as spring-damper systems (see Fig. 3.4(b)) in terms of a Voigt muscle model (Heitmann et al., 2012). The matrix $\left[f_{1}^{P}, f_{2}^{P}\right]^{T}$ of passive forces generated by $P E_{(1,2)}$ is the sum of two Hadamard products:

$$
\left[f_{1}^{P}, f_{2}^{P}\right]^{T}=\Gamma_{2 \times 1} \circ L_{2 \times 1}+\Phi_{2 \times 1} \circ V_{2 \times 1}
$$

where

- $\Gamma_{2 \times 1}$ is the matrix of stiffness parameters of $P E_{(1,2)}$, i.e., $\Gamma_{2 \times 1}=[K, K]^{T}$;

- $L_{2 \times 1}$ is the matrix of displacements of $P E_{(1,2)}$, i.e., $L_{2 \times 1}=\left[l_{1}^{P}-l_{0}, l_{2}^{P}-l_{0}\right]^{T} \cdot l_{0}$ is the initial length of $P E_{(1,2)}$;

- $\Phi_{2 \times 1}$ is the matrix of damper parameters of $P E_{(1,2)}$, i.e., $\Phi_{2 \times 1}=[D, D]^{T}$;

- $V_{2 \times 1}$ is the matrix of velocities of $P E_{(1,2)}$, i.e., $V_{2 \times 1}=\left[v_{1}^{P}, v_{2}^{P}\right]^{T}$ (see $v_{1}^{P}$ and $v_{2}^{P}$ in Fig. 3.4(a)).

The active forces produced by the CEs are approximated by the product of the neural activity $N_{j}$ and the activity strengths $i_{(1,2)}$. The matrix $\left[f_{1}^{C}, f_{2}^{C}\right]^{T}$ of the active forces generated by $C E_{(1,2)}$ (see Fig. $3.4(\mathrm{~b})$ ) is represented by:

$$
\left[f_{1}^{C}, f_{2}^{C}\right]^{T}=N_{j} \times\left[i_{1}, i_{2}\right]^{T}
$$

where

- $N_{j}$ is the neural activity of $C E_{(1,2)}$ (i.e., $N_{j} \in[-1,1]$ ). It is one of the outputs $N_{1-18}$ of the MNN (see Fig. $4.2(\mathrm{~d})$ );

- $\left[i_{1}, i_{2}\right]^{T}$ is the matrix of activity strengths for $C E_{(1,2)}$ (i.e., $\left.i_{(1,2)} \in[-1,1]\right)$. Here they are set to: $i_{1}=-i_{2}=0.5$. 


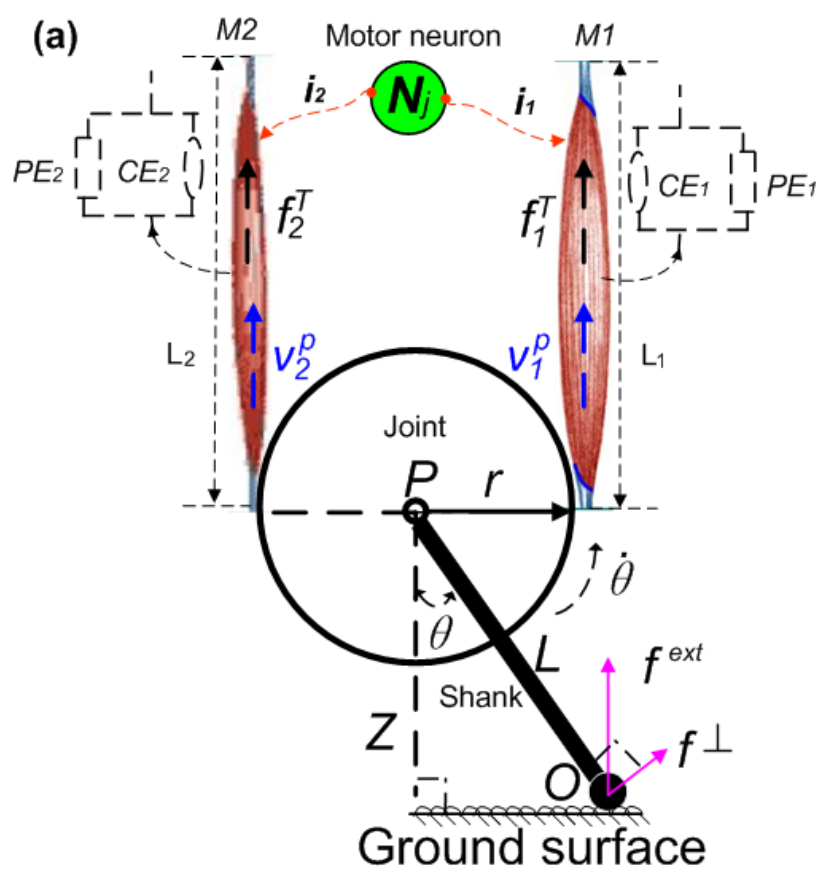

(b)

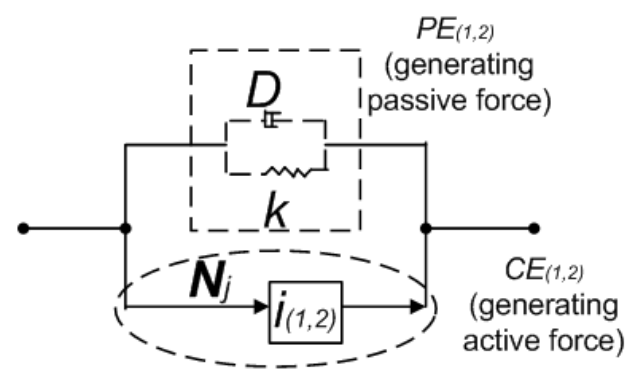

Figure 3.4: The virtual agonist-antagonist mechanism (VAAM) for joint control interacting with the ground surface. (a) The physical joint $P$ is driven by a VAAM (i.e., $M 1$ and $M 2$ ) with the lengths $L_{1}$ and $L_{2}$. The interaction results in an external force $f^{e x t}$, which drives the joint $P$ with radius $r$ via the shank with length $L$. $f^{e x t}$ is sensed by a force sensor (i.e., $O$ ), and $f^{\perp}$ is the amount of $f^{e x t}$ directly perpendicular to the position of the joint $P$. $\theta$ is the rotational angle of the joint $P$ relative to the absolute frame $Z$. (b) The agonist and antagonist mechanisms consist of contractile and parallel elements $\left(C E_{(1,2)}\right.$ and $\left.P E_{(1,2)}\right)$. $P E_{(1,2)}$ are spring-damper systems producing passive forces. $C E_{(1,2)}$ generate active forces depending on the neural activity $N_{j}$ and the activity strengths $i_{(1,2)}$ (i.e., $\left.i_{(1,2)} \in[-1,1]\right)$. The neural activity $N_{j}$ is one of the outputs $N_{1-18}$ of the modular neural network (see Fig. 4.2 (d)).

Note that the active forces generated by the CEs here are simplified to be the products of the neural activity and strengths where the length-dependent and velocitydependent functions (see Eq.(3.2)) are set to 1. This is because the simplified CEs of the VAAM receive the neural activity $N_{j}$ only to drive the joint, rather than reproduce the property-dependent functions of an insect muscle. Moreover, the VAAM can easily yield insect-like muscle functions (i.e., brakes, see Figs. 3.11 (c) and (d)) relying only on their parallel elements $P E_{(1,2)}$.

The total forces $f_{1}^{T}$ and $f_{2}^{T}$ are the sum of the active and passive forces produced 
by the muscle pair $M 1$ and $M 2$. They are given by (derived from Eqs.(3.4) and (3.5)):

$$
\begin{aligned}
& f_{1}^{T}=f_{1}^{P}+f_{1}^{C}=\underbrace{K\left(l_{1}^{P}-l_{0}\right)+D v_{1}^{P}}_{f_{1}^{P}}+\underbrace{N_{j} i_{1}}_{f_{1}^{C}}, \\
& f_{2}^{T}=f_{2}^{P}+f_{2}^{C}=\underbrace{K\left(l_{2}^{P}-l_{0}\right)+D v_{2}^{P}}_{f_{2}^{P}}+\underbrace{N_{j} i_{2}}_{f_{2}^{C}} .
\end{aligned}
$$

The antagonist mechanism M2 (see Fig. 3.4 (a)) resists the extension of the joint angle $\theta$ when receiving the force $f^{e x t}$, which is sensed by a force sensor. Simultaneously, the agonist mechanism M1 (see Fig. 3.4 (a)) produces opposing force against M2. Therefore, the directions of $f_{1}^{T}$ and $f^{e x t}$ are counter-clockwise when the direction of $f_{2}^{T}$ is clockwise. Their torques acting on the joint $P$ (see Fig. 3.4 (a)) are represented by:

$$
\begin{gathered}
\tau\left(f_{1}^{T}\right)=f_{1}^{T} r=\left(K\left(l_{1}^{P}-l_{0}\right)+D v_{1}^{P}+N_{j} i_{1}\right) r \\
\tau\left(f_{2}^{T}\right)=-f_{2}^{T} r=-\left(K\left(l_{2}^{P}-l_{0}\right)+D v_{2}^{P}+N_{j} i_{2}\right) r \\
\tau\left(f^{e x t}\right)=f^{\perp} L=f^{e x t} \sin (\theta) L
\end{gathered}
$$

where $r$ is the radius of the joint $P . f^{\perp}$ is the amount of $f^{e x t}$ directed perpendicularly to the position of the joint $P$. $L$ is the length of the shank of the joint $P$. Note that the direction of torque $\tau\left(f_{2}^{T}\right)$ is opposite to those of $\tau\left(f_{1}^{T}\right)$ and $\tau\left(f^{e x t}\right)$. We consider the torque pointing outward from the page as the positive torque (e.g., $\tau\left(f_{1}^{T}\right)$ and $\left.\tau\left(f^{e x t}\right)\right)$.

We apply Euler's law to the rotation of the joint $P$ (see Fig. 3.4(a)). The net torque $\sum \tau$ acting on the joint $P$ is equal to the product of its moment of inertia $I$ and angular acceleration $\ddot{\theta}$. It is given by:

$$
I \ddot{\theta}=\sum \tau=\tau\left(f^{e x t}\right)+\tau\left(f_{1}^{T}\right)+\tau\left(f_{2}^{T}\right) .
$$

Derived from Eq.(3.11) (see more details in Appendix A.1), the motion equation of the joint $P$ is given by:

$$
I \ddot{\theta}=\underbrace{f^{e x t} \sin (\theta) L}_{\text {torque by } f^{e x t}}+[\underbrace{r N_{j}}_{\text {torque by } f_{(1,2)}^{C}}-\underbrace{2 r^{2}(K \theta+D \dot{\theta})}_{\text {torque by } f_{(1,2)}^{P}}] .
$$

Equation (3.12) governs the angle $\theta$ of a physical joint driven by the VAAM that is activated by the output $N_{j}\left(j \in \mathbb{Z}_{[1,18]}\right)$ of the MNN (see the values of $I$ and $r$ at Appendix A.2). The joint angle $\theta$ and joint velocity $\dot{\theta}$ in Eq.(3.12) are not from sensory feedback but calculated using fourth-order RungeKutta. 


\subsection{Variable compliance control for hexapedal stand- ing and walking}

Here, we first investigate compliant joint control driven only by the passive elements (i.e., $\left.P E_{(1,2)}\right)$ of the VAAM. Thus setting the neural output $N_{j}$ to 0 in Eq.(3.12), the compliant joint control is given by:

$$
I \ddot{\theta}=\underbrace{f^{e x t} \sin (\theta) L}_{\text {torque by } f^{e x t}}-\underbrace{2 r^{2}(K \theta+D \dot{\theta})}_{\text {torque by } f_{(1,2)}^{P}} .
$$

Equation (3.13) shows that the VAAM relies only on its passive elements (i.e., $\left.P E_{(1,2)}\right)$ to achieve variable compliance control (see Fig 3.5). In principle, the VAAM shares a connection to classical impedance control approaches (Hogan and Buerger, 2004; Hwangbo et al., 2014) based on proportional-derivative (PD) control (see Eq.(2.1)) (Tomei, 1991; Luca et al., 2005).

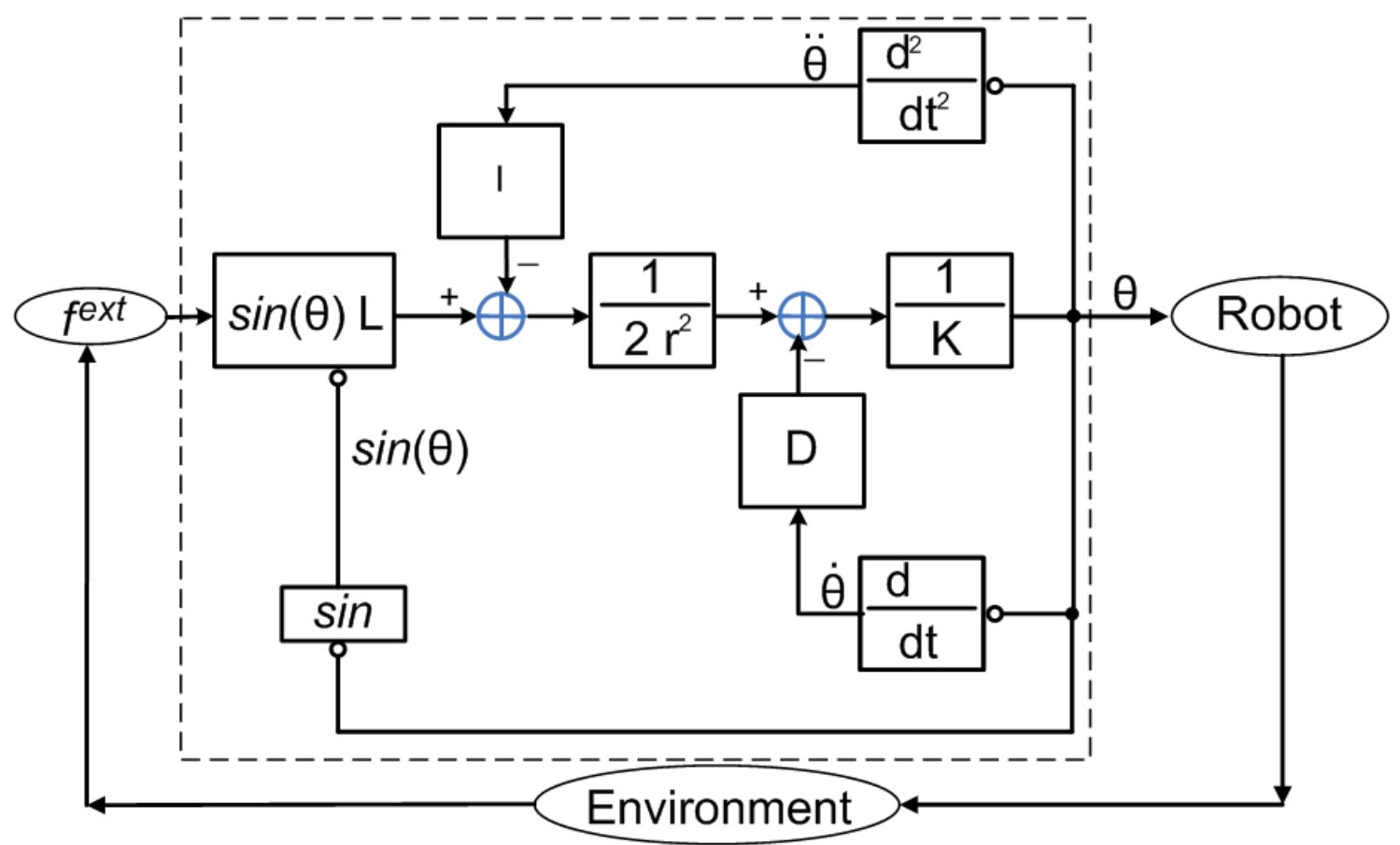

Figure 3.5: Compliant joint control based on the parallel elements $P E_{(1,2)}$ of the VAAM. The controller produces joint angle $\theta$ after receiving exciting force feedback $f^{e x t}$, which arises by the interactions with the environment. Variable compliant joint motions can be produced adjusting the stiffness and damper parameters (see $K$ and $D$ in Eq.(3.13)) of $P E_{(1,2)}$. 
(a)

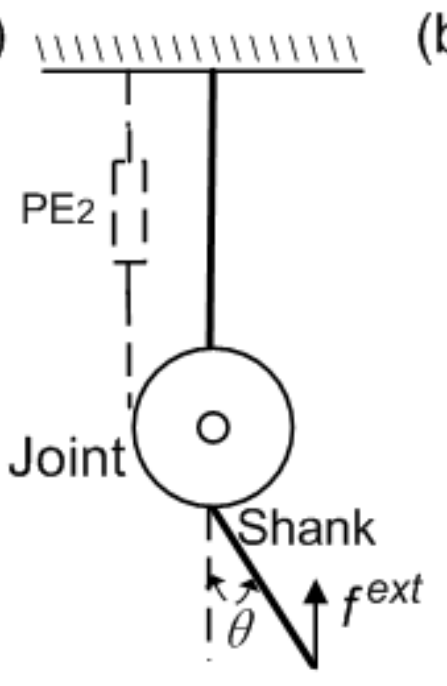

(b)

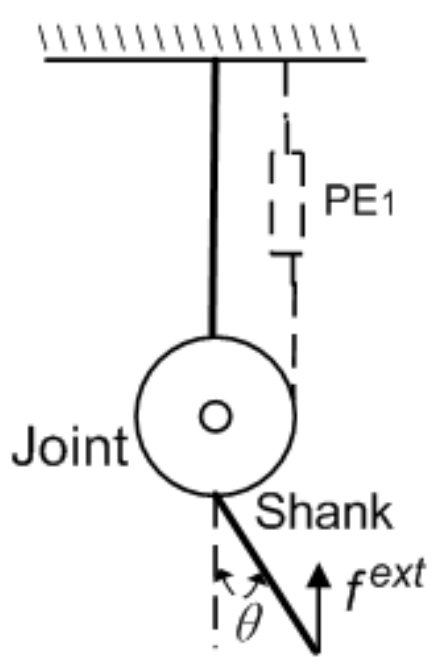

(c)

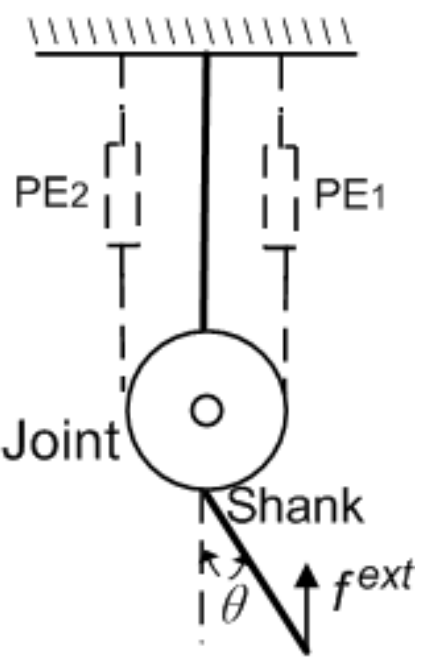

Figure 3.6: A comparison of different configurations of the parallel element (PE). (a) Only $P E_{2}$. (b) Only $P E_{1}$. (c) A pair of $P E_{(1,2)}$. All $P E_{(1,2)}$ mentioned above have the same stiffness and damper parameters, i.e., $K=0.8, D=1.0$.

Generally, the single spring-damper system (see Fig. 3.6 (a) or (b)) is applied to PD control of the joint (see Eq.(2.1)). Unlike the classical PD controllers, the VAAM is the bio-inspired controller where a pair of the spring-damper systems (see Fig. 3.6 (c)) is used as its passive elements that emulate passive properties of the agonist and antagonist muscles. That is because a pair of agonist and antagonist mechanisms (see Fig.3.6 (c)) acting in concert can generate more power than the sum of them acting individually (i.e., a single agonist or antagonist mechanism, see Fig. 3.6 (a) or (b)) when receiving a certain load (Farahat and Herr, 2010). In addition, a pair of the PEs (Fig. 3.6 (c)) allows for faster joint stability, compared to a single agonist or antagonist mechanism (see Figs. 3.6 (a) and (b)). Specifically, the control of the single agonist/antagonist follows as (rewriting Eq.(3.13)):

$$
I \ddot{\theta}=f^{e x t} \sin (\theta) L-r^{2}(K \theta+D \dot{\theta}) .
$$

One can see that $P E_{1}$ or $P E_{2}$ leads to slower joint stability than when the joint is driven by a pair of $P E_{1}$ and $P E_{2}$ (see Figs. 3.7). Thus, a pair of the PEs outperforms the other configurations by producing fast stabilizing joint movement. Moreover, such a pair of the PEs produces smooth and compliant joint motions to well fight against noisy exciting forces (e.g., see Figs. 3.7). Actually similar compliant joint motions can be also achieved using admittance/impedance controllers (Yang et al., 2011) or VSAs (Variable Stiffness Actuators) (Ham et al., 2009). In general, the VSAs are based on the concepts of the SEAs (Serial Elastic Actuators) (Pratt et al., 2002). For instance, a VSA with variable physical dampers can be tuned within a 


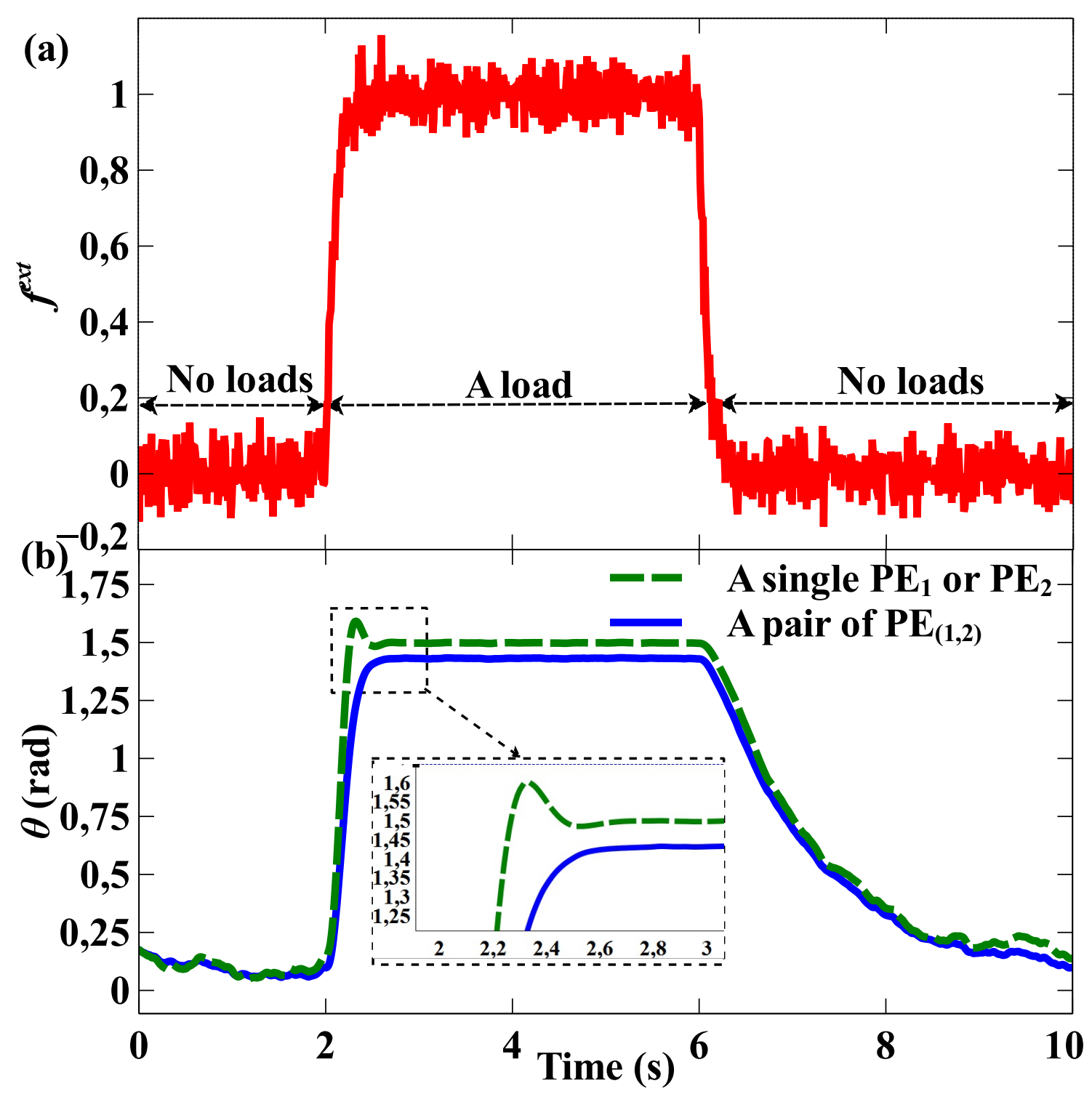

Figure 3.7: Stability comparison. (a) Exciting and noisy force $f^{e x t}$. (b) Joint angles $\theta$. Green line: $P E_{1}$ or $P E_{2}$; blue line: a pair of $P E_{(1,2)}$. Initial value $\left[\theta_{0}, \dot{\theta_{0}}\right]=$ $[0.175(\mathrm{rad}), 0(\mathrm{rad} / \mathrm{s})]$. The joint driven by the pair of $P E_{(1,2)}$ smoothly reaches faster stability than when it is driven by only $P E_{1}$ or $P E_{2}$ (see the three setups in Figs. 3.6). All PEs have same stiffness and damper parameters $[K, D]$, i.e. $[K, D]=[0.8,1.0]$. Interestingly, this parameter setup makes a pair of $P E_{(1,2)}$ generate the muscle-like functions (i.e., smooth brakes, see Fig. 3.10 (b)), which are comparable to those of biological muscles (Ahn and Full, 2002; Full et al., 1998). By contrast, only $P E_{1}$ or $P E_{2}$ cannot produce the smooth brake (see Fig. 3.10 (a)). 
wide range of impedance through admittance control (Laffranchi et al., 2011). However, the hardware of the VSAs is too bulky and complex to be applied to small robotic platforms (less than $8 \mathrm{~kg}$ weight (Galloway, 2010)) (Semini et al., 2013; Hurst et al., 2010). Whereas variable admittance/impedance controllers ${ }^{2}$ can reduce hardware complexities using software control of joint compliance (Albu-Schäffer et al., 2011; Buchli et al., 2011). Generally, variable compliant joint motions are achieved adjusting the stiffness and damper parameters of the virtual spring-damper systems that replace physical springs and dampers (e.g., see Eq.(2.1)) (Hogan and Buerger, 2004; Hwangbo et al., 2014). Such joint motions enable robots to behave variable soft interactions against different perturbations (e.g., hand pushing). In addition, they make the robots return to original postures when the perturbations disappear. For example, Stephens and Atkeson developed a predictive model relying on force/torque feedback on each joint of the Sarcos humanoid robot (80 kg weight) where each joint is driven by a hydraulic actuator (Stephens and Atkeson, 2010a). The model allows the humanoid robot to stabilize its body after perturbations are removed (Stephens and Atkeson, 2010b). Besides, Havoutis et al. used virtual model control for the quadruped robot HyQ (90 kg weight), which is a fully torque-controlled hydraulically actuated robot. The virtual model controller relies on force/torque feedback on each joint of the robot (I. Havoutis and Caldwell, 2012). However, such controllers rely heavily on complex force/torque sensing at each joint of the system ( $\mathrm{Yu}$ et al., 2011), which are still hard to be applied to small robotic platforms (less than $8 \mathrm{~kg}$ weight (Galloway, 2010)). Their performances also depend heavily on the accuracy of additional sensory (i.e., position) feedback (Sensinger, 2010; Ott et al., 2010). By contrast, our proposed virtual agonist-antagonist mechanisms (VAAMs) enable the driven joints to produce variable compliant motions relying only on force sensing at the end effector of each leg, rather than force/torque feedback on each joint. This is because the required angle feedback and velocity feedback of each joint are not directly from sensory feedback but approximated using fourth-order RungeKutta to solve Eq.(3.12). Moreover, compliant joint control based on the VAAMs is more bio-inspired control that leads to easily emulating muscle-like functions, compared to classical admittance/impedance controllers (I. Havoutis and Caldwell, 2012; Ott et al., 2010). Specifically, the VAAMs facilitate easily adjusting the stiffness and damper parameters of their passive elements (i.e., $P E_{(1,2)}$ ) to emulate muscle-like functions, by novelly using the work loop technique. The technique, prevailing in muscle physiology, can be used to assess the mechanical work and power output of musculoskeletal contractions via in-vitro ${ }^{3}$ muscle tests (Biewener, 2003; Ahn, 2012).

\footnotetext{
${ }^{2}$ Here admittance control is defined as a control method that receives force inputs and generates motion outputs while impedance control is defined as a control method that receives motion inputs and generates force outputs (Ott et al., 2010).

${ }^{3}$ In-vitro muscle testing is an approach used to characterize properties of living muscle tissues that are removed from an organism.
} 
Using the technique, the muscle force and length are measured when applying an external load. In our implementation, the external load $f^{\text {ext }}$ (see Fig. 3.8) is applied to the joint driven only by the passive elements (i.e., $P E_{(1,2)}$ ) of the VAAMs (see Eq.(3.13)).

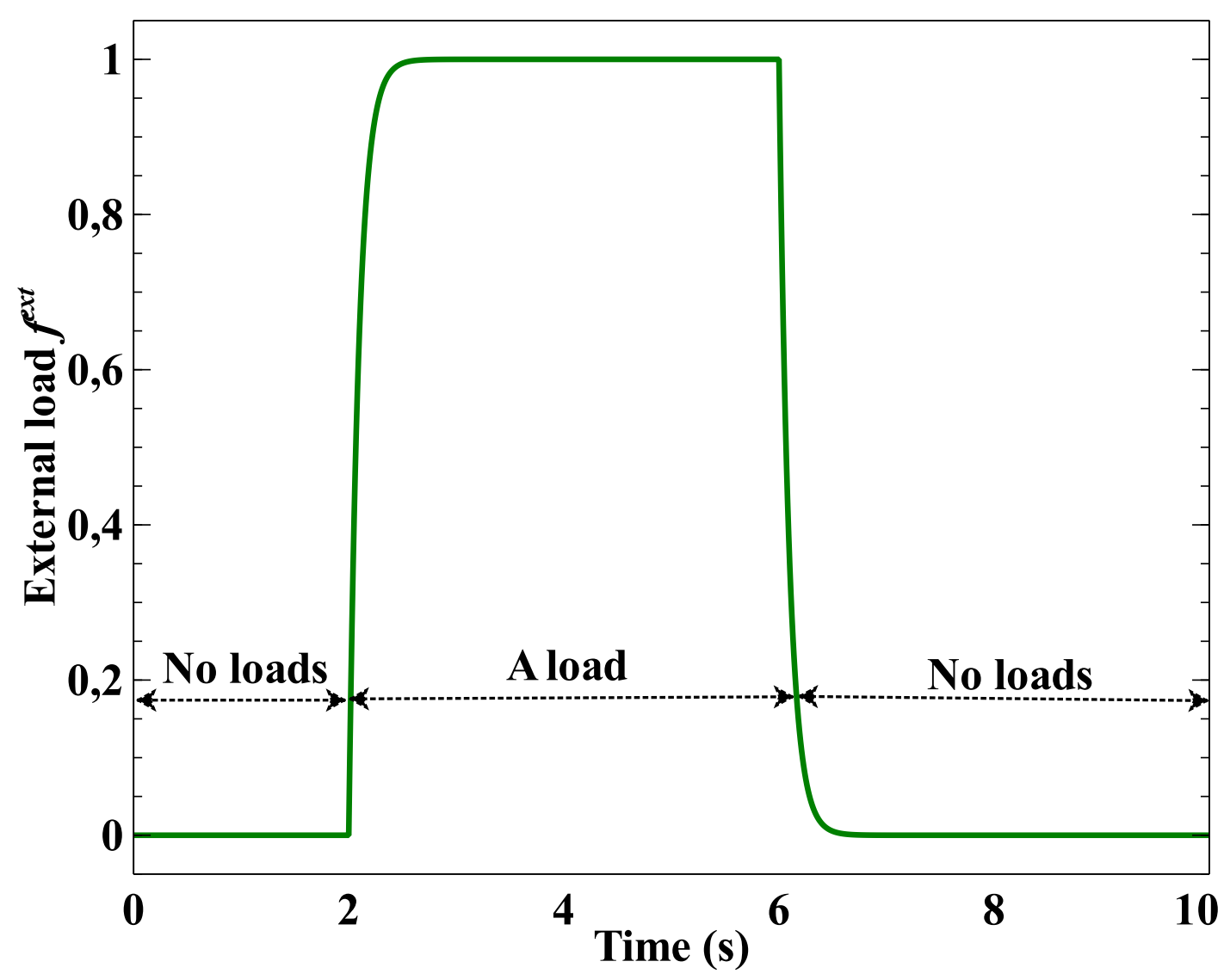

Figure 3.8: The external load $f^{e x t}$.

Excited by the external load $f^{e x t}, P E_{(1,2)}$ of the VAAMs yield the passive forces $f_{(1,2)}^{P}$ that are given by (derived from Eq.(3.4)):

$$
f_{(1,2)}^{P}=\mp(K \theta r+D \dot{\theta} r)
$$

where the angle $\theta$ and velocity $\dot{\theta}$ are calculated from Eq.(3.13) using fourth-order RungeKutta. In addition, the lengths $l_{(1,2)}^{P}$ of $P E_{(1,2)}$ satisfy (derived from Eq.(A.4)):

$$
l_{(1,2)}^{P}=l_{0} \mp(\theta r),
$$

where $l_{0}$ denotes the initial length of $P E_{(1,2)}$, and $r$ is the radius of the joint that is driven only by $P E_{(1,2)}$ of the VAAM (see their values at Appendix A.2). The work loop technique makes the VAAMs easily quantify their resulting muscle-like functions 
that accommodate different contexts (e.g., standing or walking). For instance, when our insect-like robot AMOS stands against the perturbations, the damper parameter $D=0.01$ or $D=0.05$ is better for AMOS to reduce leg contact force (see Fig. 3.15) or achieve faster joint stabilizations (see Fig. 3.17 (c)) showing the muscle-like functions (i.e., springs or dampers). As the springs, the muscle-like mechanisms $P E_{(1,2)}$ are able to produce negative work by fast swinging between states of compliant motions (e.g., see Figs. 3.17 (a) and (b)). In addition, the muscle-like mechanisms $P E_{(1,2)}$ act as the dampers to absorb energy from the perturbed load (e.g., see Fig. 3.17 (c)), which leads to faster joint stabilization. As the dampers, the muscle-like mechanisms $P E_{(1,2)}$ absorb energy in a more oscillatory way (see $D=0.05$ in Figs. 3.9) during perturbed legged standing. By contrast, stable legged walking requires the muscle-like mechanisms $P E_{(1,2)}$ to serve as stronger absorbers (i.e., brakes) that stably shorten and lengthen due to more dynamic imposed loads. In insect locomotion, for example, their muscles often serve as the brakes (i.e., stronger load absorbers) such that they can stably absorb energy from more dynamic imposed loads (Dickinson et al., 2000; Ahn and Full, 2002). To emulate such brakes, $P E_{(1,2)}$ need to stably absorb energy from the perturbed loads. In the absorption, $P E_{(1,2)}$ shorten with the decreasing force outputs (e.g., see $P E_{1}$ in Fig. 3.10 (b)). After the absorption, $P E_{(1,2)}$ stably lengthen and return back to the initial lengths due to being the brakes. Thus the force-length loops of the brakes are characterized as the clockwise and closed forcelength loops. Since the damper parameter $D=0.05$ is too lower to make $P E_{(1,2)}$ act as the stronger absorbers (i.e., brakes), the damper parameter of $P E_{(1,2)}$ needs to be increased to emulate the brakes for insect-like walking. For example, increasing the damper parameter $D$ to 1.0 enables $P E_{(1,2)}$ to act as the brakes that stably lengthen and shorten when the load is applied and removed (see $D=1.0$ in Figs. 3.9). The stable lengthening and shortening behaviors are characterized by the clockwise and closed force-length loops (see Fig. 3.10 (b)). Interestingly, such force-length loops of the muscle-like mechanisms (i.e., $\left.P E_{(1,2)}\right)$ are comparable to those of insect muscles (Dickinson et al., 2000; Full et al., 1998; Ahn and Full, 2002). Therefore, fixing the damper parameter $D$ of $P E_{(1,2)}$ to 1.0 is better for AMOS to achieve insectlike walking due to being stronger absorbers (i.e., brakes). Note that the stiffness parameter $K$ of $P E_{(1,2)}$ is set to 0.8 in these brakes (see their force-length loops in Fig. 3.10 (b)). In addition, adjusting the stiffness parameter $K$ of $P E_{(1,2)}$ of the VAAMs will present different loops when their damper parameter is fixed to 1.0. For example, lower (see Figs. 3.11 (a) and (b)) or higher (see Figs. 3.11 (e) and (f)) stiffness parameters cannot make $P E_{(1,2)}$ produce clockwise and closed force-length loops.

Whereas $P E_{(1,2)}$ can present clockwise and closed force-length loops when their stiffness parameter $K$ is set to 3.0 or 4.0 (see Figs. 3.11 (c) and (d)). This is because the stiffness parameter $K=3.0$ or $K=4.0$ enables $P E_{(1,2)}$ to appropriately produce soft lengthening and shortening behaviors (e.g., returning the original length), which leads to closing the force-length loops (see $K=3.0$ and $K=4.0$ in Fig. 3.12). There- 


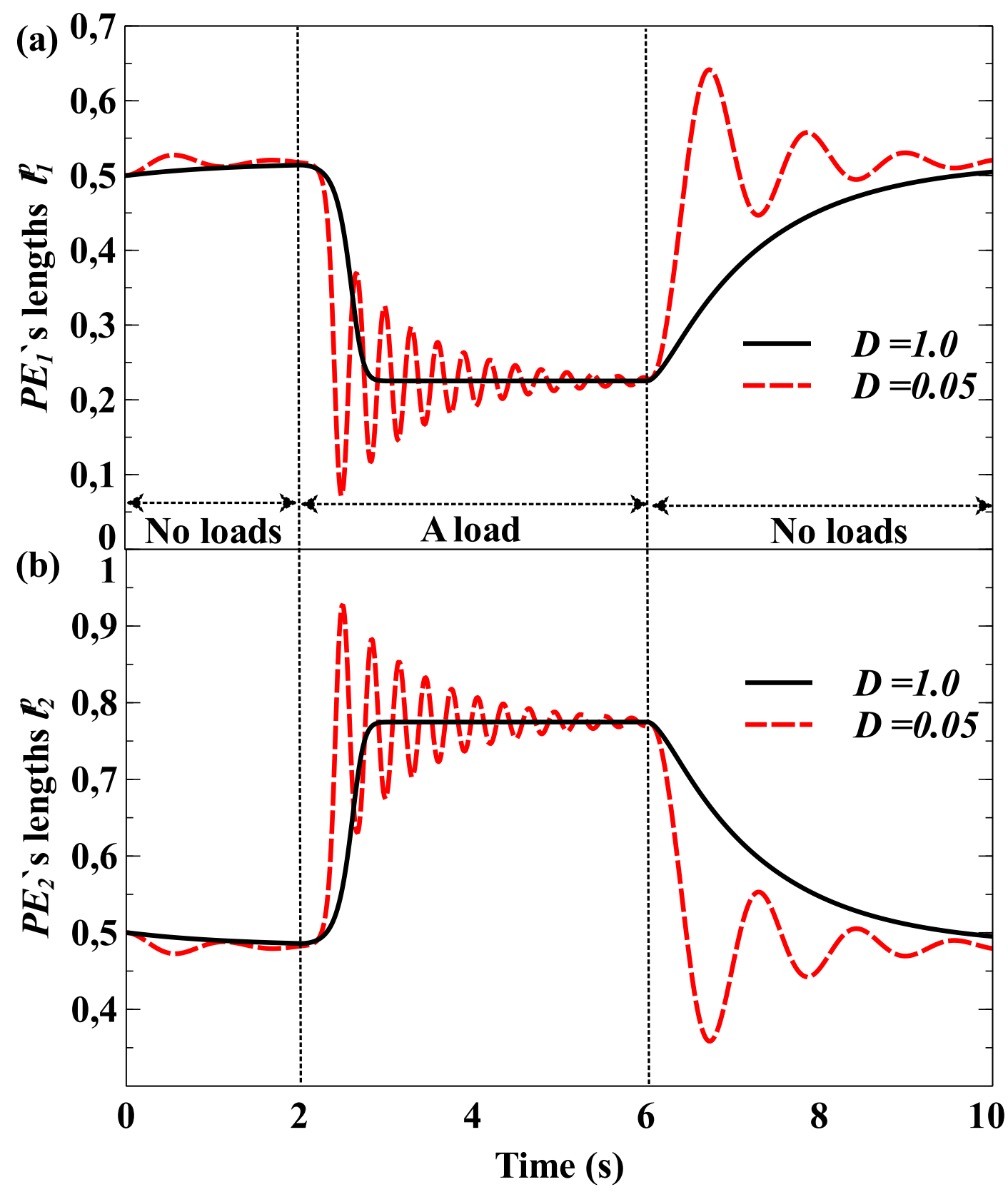

Figure 3.9: The lengths of $P E_{(1,2)}$ of the VAAMs with different damper parameters. Here the stiffness parameter $K$ is set to 0.8. (a) $P E_{1}$ 's lengths $l_{1}^{P}$. (b) $P E_{2}$ 's lengths $l_{2}^{P}$. Once can see that the damper parameter $D=0.05$ makes $P E_{(1,2)}$ act as the dampers that absorb energy from the load in a more oscillatory way. Whereas increasing the damper parameter $D$ to 1.0 enables $P E_{(1,2)}$ to serve as the brakes, which stably absorb loads. 


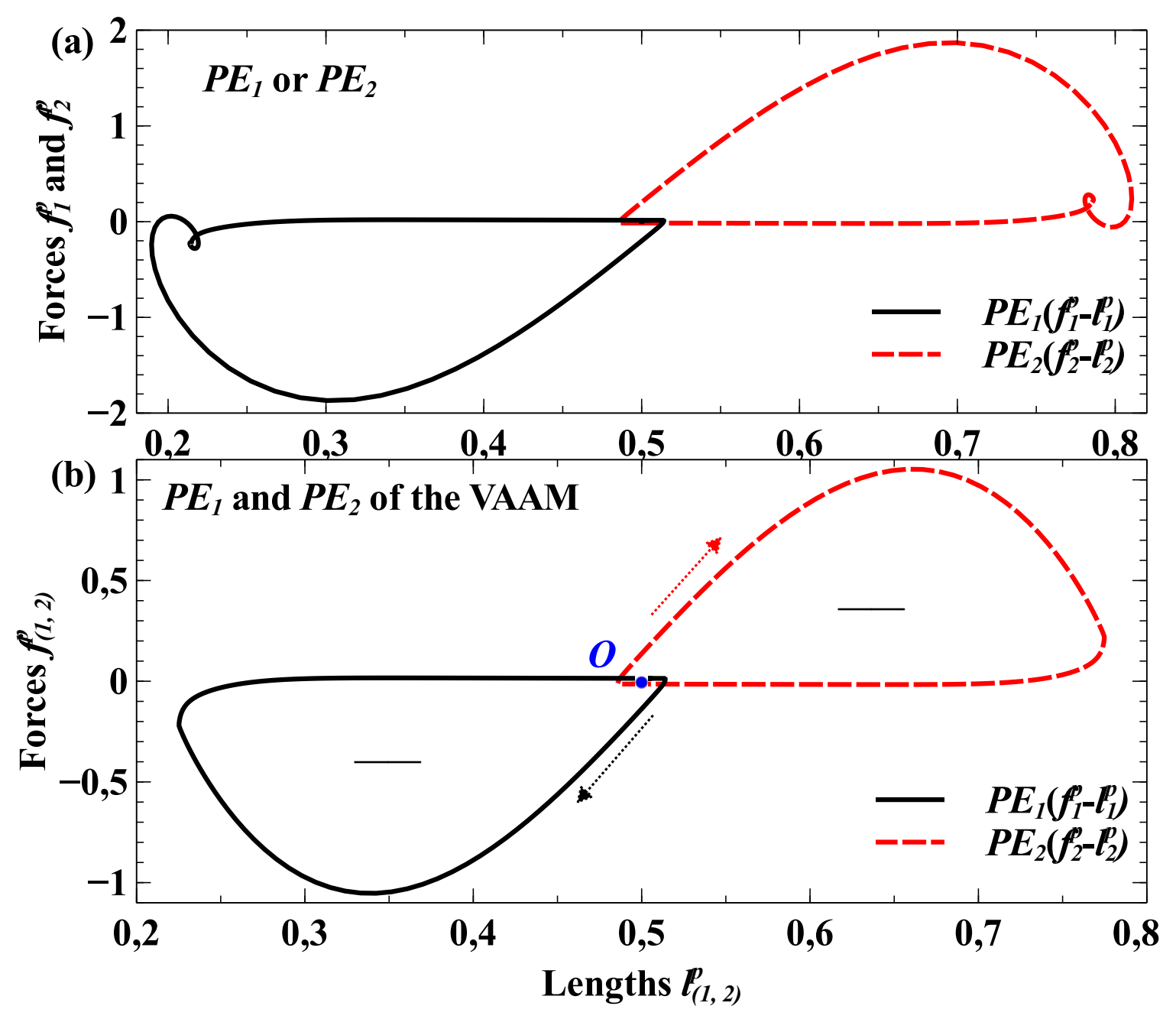

Figure 3.10: The force-length loops of $P E_{(1,2)}$ and the VAAM with the stiffness and damper parameters $K=0.8, D=1.0$. The forces $f_{(1,2)}^{P}$ and lengths $l_{(1,2)}^{P}$ satisfy Eqs.(3.15) and (3.16). (a) Only $P E_{2}$ or $P E_{1}$ (see Figs. 3.6 (a) and (b)). Only $P E_{2}$ or $P E_{1}$ doesn't stably absorb energy from the load (see Fig. 3.7 (b)). (b) the VAAM (see Fig. $3.6(\mathrm{c})$ ). Its $P E_{(1,2)}$ act as the brakes that stably absorb energy from the load (see $D=1.0$ in Figs. 3.9). $O$ is the starting point of the force-length loops. Note the symbols '-' inside the clockwise loops mean $P E_{(1,2)}$ act as the brakes that result in negative work. Here the applied load is $f^{e x t}$ shown in Fig. 3.8.

fore, such intermediate stiffness parameters enable $P E_{(1,2)}$ to act as the brakes that properly absorb energy from the load during perturbed hexapedal walking. Whereas lower (e.g., $K=0.1$ ) or higher (e.g., $K=7.0$ ) stiffness parameters cannot make $P E_{(1,2)}$ return their original lengths (see the dotted and darked lines in Fig. 3.12), 


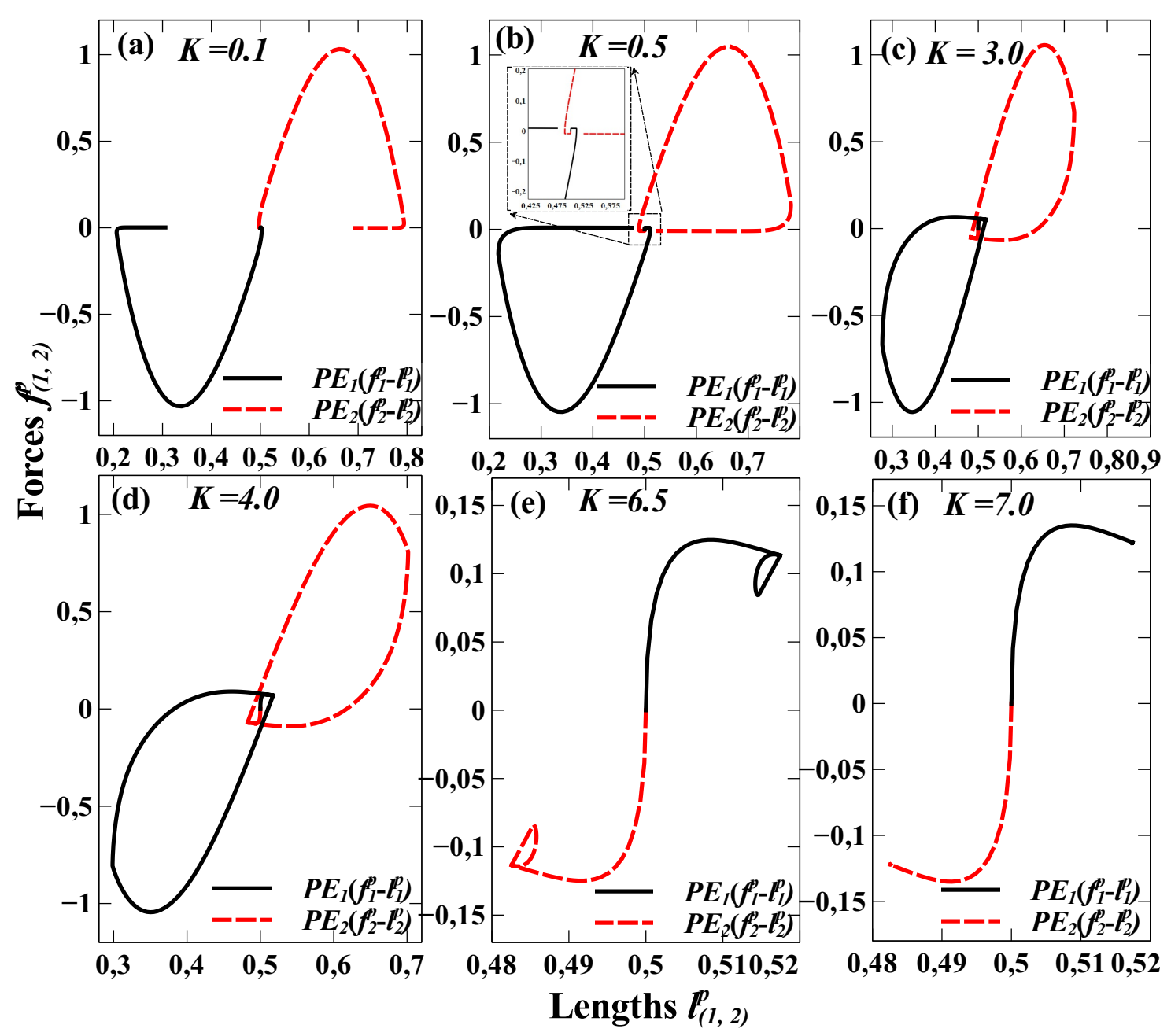

Figure 3.11: The force-length loops of $P E_{(1,2)}$ of the VAAMs varies with different stiffness parameters $K$. (a) $K=0.1$. (b) $K=0.5$. (c) $K=3.0$. (d) $K=4.0$. (e) $K=6.5$. (f) $K=7.0$. Figures (c) and (d) show the clockwise and closed forcelength loops that are referred as the brakes. These muscle-like functions of $P E_{(1,2)}$ are comparable to those of insect muscles during their walking (Dickinson et al., 2000; Full et al., 1998; Ahn and Full, 2002). Here the damper parameter $D$ is set to 1, and the applied load is $f^{e x t}$ shown in Fig. 3.8.

which results in low or high stiff joint motions. Note that "high stiff" (i.e., stiffer) here means that a joint greatly resists the influence of external forces (e.g., see $K=7.0$ in Fig. 3.12), thereby leading to low joint compliance (Pratt, 2000, 2002). By contrast, "low stiff" (i.e., softer) here means that a joint allows external forces to influence its movement easily (e.g., see $K=0.1$ in Fig. 3.12), thereby resulting in high joint compliance (Pratt, 2002; Pratt et al., 2002). One can see that the joint driven by 


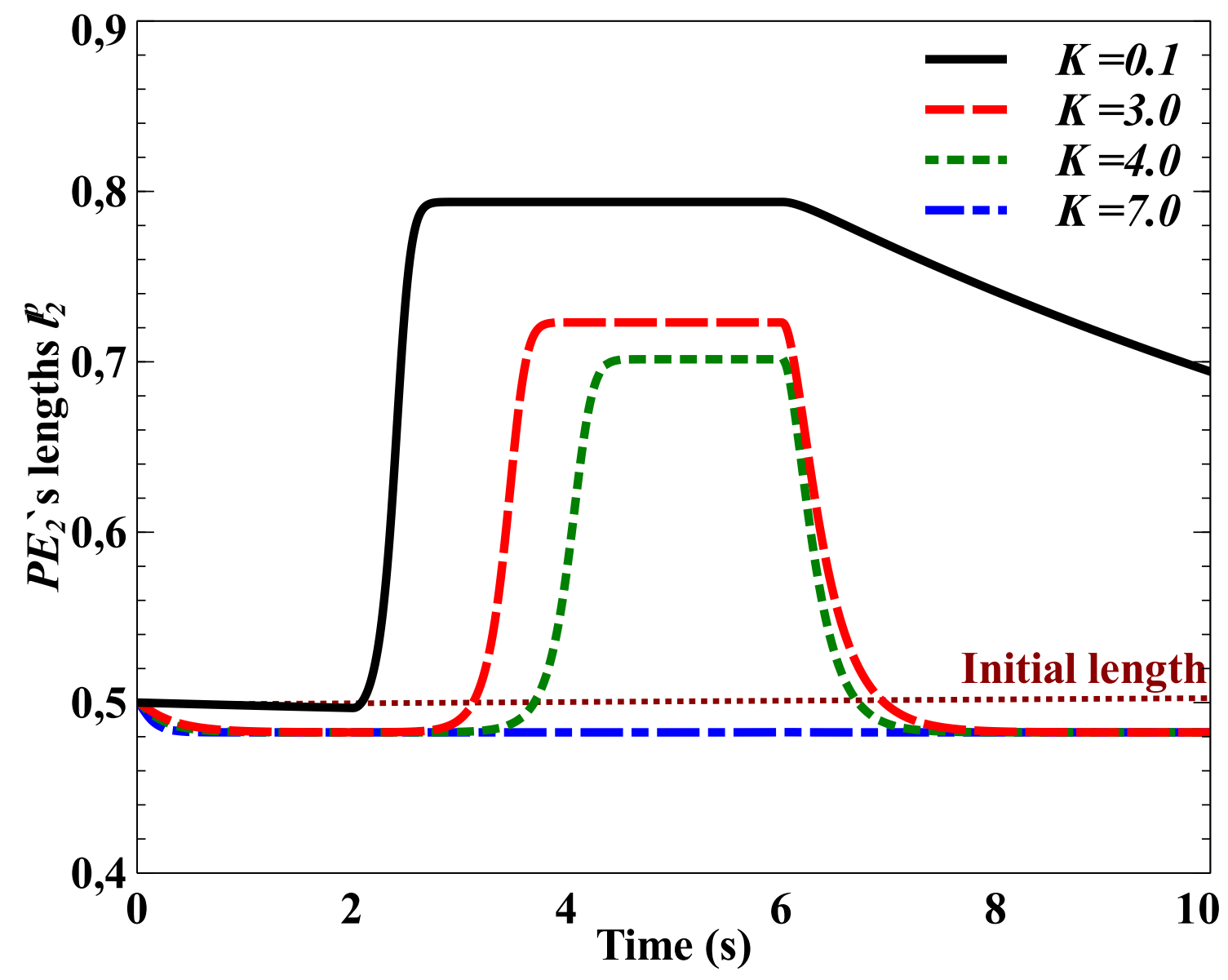

Figure 3.12: $P E_{2}$ 's lengths $l_{2}^{P}$ that vary with stiffness parameters $K . K=3.0$ or $K=4.0$ makes $P E_{2}$ appropriately generate soft lengthening and shortening behaviors (e.g., return to initial lengths), compared to lower (e.g., $K=0.1$ ) or higher (e.g., $K=7.0)$ stiffness parameters. Here their damper parameters $D$ are set to 1 , and the applied load is $f^{\text {ext }}$ shown in Fig. 3.8.

$P E_{(1,2)}$ produces intermediate compliant motions when the stiffness and damper parameters are set as: $K=3$ or $K=4$, and $D=1.0$ (see $K=3$ and $K=4$ in Fig. 3.12). These two setups enable $P E_{(1,2)}$ to serve as the brakes, which are comparable to those of biological insect muscles (Ahn and Full, 2002). Such insect-like muscle functions (i.e., brakes) are better for our insect-like robot AMOS to stably and compliantly fight against the perturbations during its walking on rough surfaces (e.g., gravel). Thus the two setups are chosen to achieve stable and variable compliant hexapedal walking on rough surfaces (see Table 3.1). By contrast, for variable compliant standing, AMOS needs to produce more compliant joint motions (e.g., see $D=0.05$ in Figs. 3.9) to handle static and dynamic loads (e.g., hand pushing). Therefore, the three values of the damper parameter $D$ will be chosen for AMOS's standing to fight against the 
Table 3.1: The setups of stiffness and damper parameters $[K, D]$ in hexapedal standing and walking

\begin{tabular}{|c|c|c|}
\hline $\begin{array}{l}\text { Setups and functions } \\
\text { Applications }\end{array}$ & {$[K, D]$} & Muscle-like functions \\
\hline Standing & $\begin{array}{c}{[0.8,0.001]} \\
{[0.8,0.01], \text { and }} \\
{[0.8,0.05]}\end{array}$ & $\begin{array}{l}\text { springs and dampers } \\
\text { (see Figs. 3.17) }\end{array}$ \\
\hline Walking & $\begin{array}{l}{[3.0,1.0] \text { and }} \\
{[4.0,1.0]}\end{array}$ & $\begin{array}{l}\text { brakes (see Figs. } 3.11 \\
\text { (c) and }(\mathrm{d}) \text { ) }\end{array}$ \\
\hline
\end{tabular}

loads when the stiffness parameter $K$ is set to 0.8 (see Table 3.1). To our knowledge, the model of the VAAMs is the first bio-inspired muscle model that novelly utilizes the work loop technique to quantify muscle-like functions underlying variable compliant joint motions. As a result, such a quantification facilitates easier emulations of muscle-like functions and their applications (e.g., standing) on compliant joint control (see Table 3.1).

\subsection{Variable compliant hexapedal standing against loads}

The variable compliance control is applied to the FTi and CTr joints of AMOS based on the PEs of the VAAMs. Specifically, each of them is driven by a pair of $P E_{(1,2)}$ of the VAAM (see Fig. 3.13). Note that the TC joint of the leg allowing only horizontal motion is not affected by the PEs of the VAAM, since there is only detection of vertical foot force at the end effector of the leg. Thus the joint control of a two-jointed leg follows as (rewrite Eq.(3.13)):

$$
\ddot{\theta}_{(2 \times 2)} I_{(2 \times 1)}=f^{e x t} \vec{V}_{(2 \times 1)}-2 r^{2}\left(\theta_{(2 \times 2)} K_{(2 \times 1)}+\dot{\theta}_{(2 \times 2)} D_{(2 \times 1)}\right),
$$

where its matrices are:

$$
\begin{gathered}
\ddot{\theta}_{(2 \times 2)}=\left[\begin{array}{cc}
\ddot{\theta}_{1} & 0 \\
0 & \ddot{\theta}_{2}
\end{array}\right], I_{(2 \times 1)}=\left[\begin{array}{l}
I \\
I
\end{array}\right], \\
\vec{V}_{(2 \times 1)}=\left[\begin{array}{c}
\vec{V}_{1} \\
\vec{V}_{2}
\end{array}\right], \quad \vec{V}_{1}=L_{1} \sin \left(\theta_{1}\right) \\
\vec{V}_{2}=L_{2} \cos \left(\theta_{2}\right)+\vec{V}_{1}, \\
\theta_{(2 \times 2)}=\left[\begin{array}{cc}
\theta_{1} & 0 \\
0 & \theta_{2}
\end{array}\right], K_{(2 \times 1)}=\left[\begin{array}{c}
K \\
K
\end{array}\right], \\
\dot{\theta}_{(2 \times 2)}=\left[\begin{array}{cc}
\dot{\theta}_{1} & 0 \\
0 & \dot{\theta}_{2}
\end{array}\right], D_{(2 \times 1)}=\left[\begin{array}{c}
D \\
D
\end{array}\right] .
\end{gathered}
$$


The values of $I$ and $L_{(1,2)}$ are fixed and can be seen at Appendix A.2. Changing the stiffness and damper parameters in $K_{(2 \times 1)}$ and $D_{(2 \times 1)}$ enables AMOS to generate variable compliant joint motions that fight against different loads. As a result, such compliant joint motions lead to friendly robotic interactions (e.g., the preventions of the damages) with different environments.

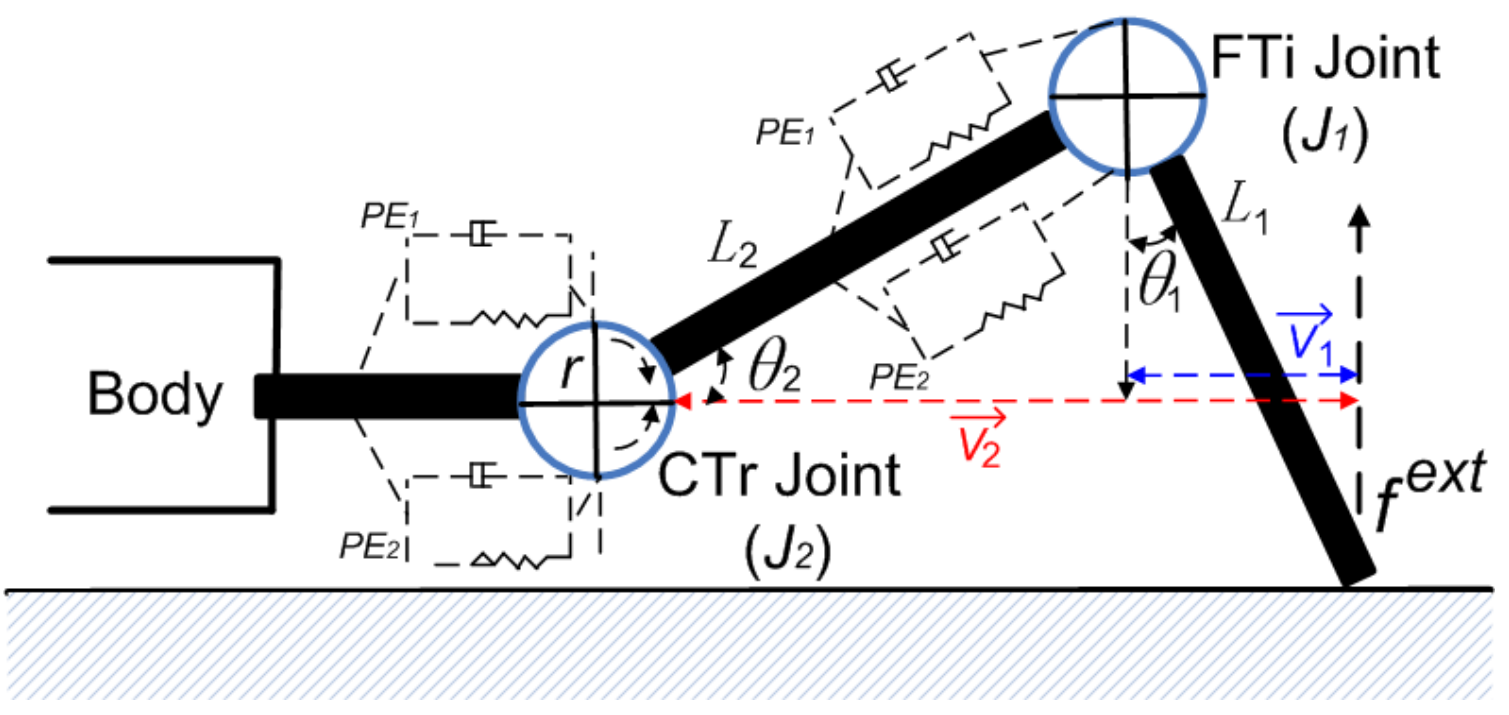

Figure 3.13: Variable compliant control of a two-joint leg based on the PEs of the VAAMs. Each joint (i.e., $\left.J_{(1,2)}\right)$ is driven by a pair of $P E_{(1,2)}$ of the VAAM. After the exciting force $f^{e x t}$ is imposed, the two joint angles $\theta_{1}$ and $\theta_{2}$ vary with the different stiffness and damper parameters of $P E_{(1,2)}$ (see Eqs.(3.20) and (3.21)). The lengths of the links are $L_{1}$ and $L_{2} . \vec{V}_{1}$ and $\vec{V}_{2}$ are the displacement vectors of $J_{(1,2)}$, which $f^{e x t}$ directly or indirectly acts on.

\subsubsection{Reducing impact force against a static load}

In the experimental setup (see Fig. 3.14), AMOS was placed between supporters having a total height of $18 \mathrm{~cm}$ (i.e., Ls $=18(\mathrm{~cm})$ ). Due to its height $22 \mathrm{~cm}$, AMOS carried the load when we placed a board (i.e., a static load) on top of it. Thus, AMOS's legs have to resist the load when the passive springs fail (i.e., they cannot be compressed any more). Here the suffered impact forces $F C_{1-4}$ at legs are used to measure the performances of four setups, three of which are empirically adjusted stiffness and damper parameters of $P E_{1,2}$ (see Eq.(3.17) and Table 3.2). $F C_{1-4}$ are given by:

$$
F C_{1-4}=\sum_{m=1}^{6} f c_{m, 1-4}
$$




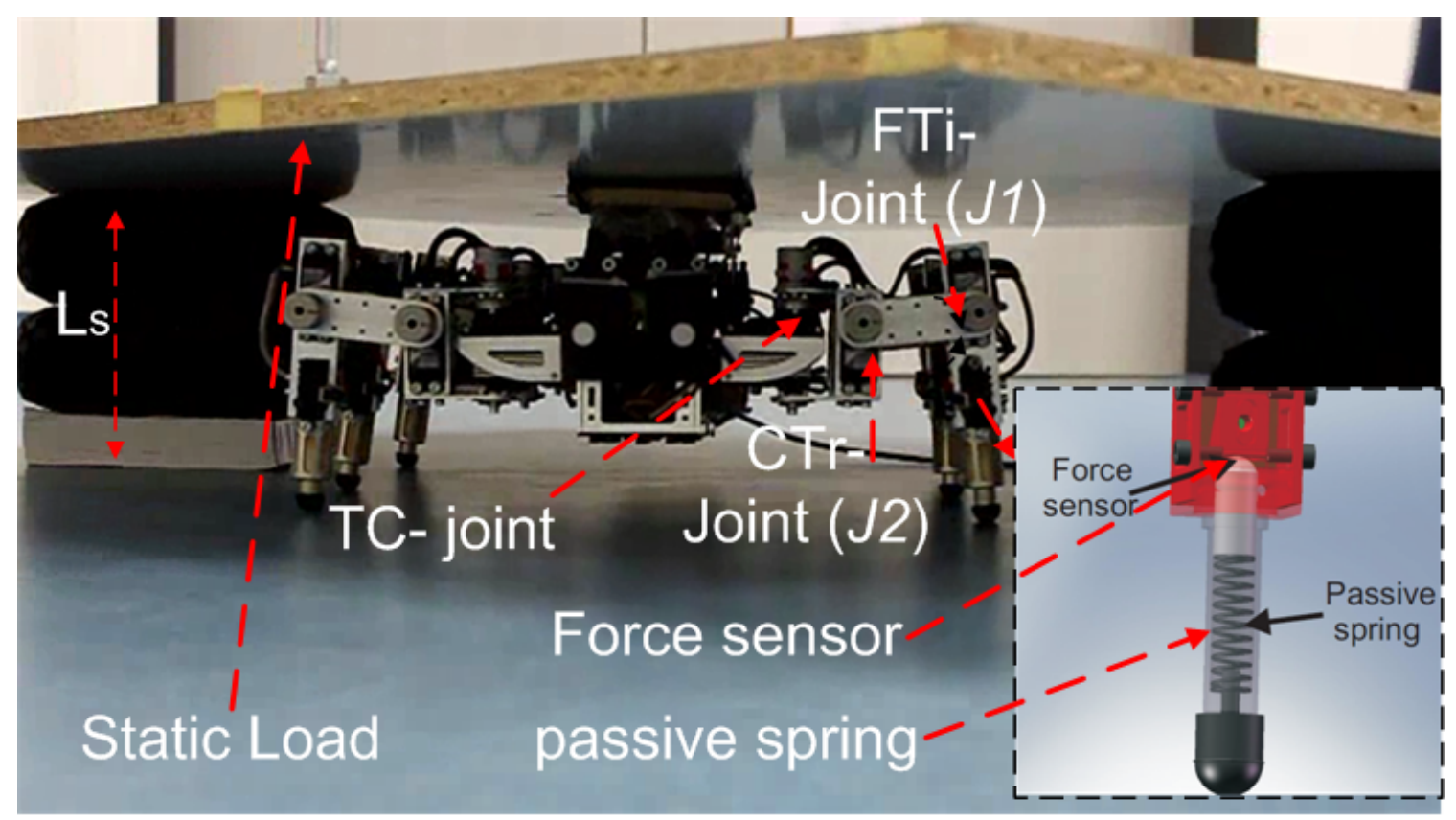

Figure 3.14: The experimental setup of AMOS against a static load. Here AMOS's $\mathrm{CTr}$ and FTi joints are driven by the pairs of $P E_{1,2}$ (see Fig. 3.13). More details of the setup can be seen at the texts.

Table 3.2: Three stiffness and damper parameter setups of $P E_{1,2}$

\begin{tabular}{|c|c|c|c|}
\hline $\begin{array}{lr}\text { Stiffness and damper parameters } & \text { Setups } \\
\end{array}$ & $\mathrm{S} 1$ & $\mathrm{~S} 2$ & $\mathrm{~S} 3$ \\
\hline Damper parameters $D$ & 0.001 & 0.01 & 0.05 \\
\hline Stiffness parameters $K$ & 0.8 & 0.8 & 0.8 \\
\hline
\end{tabular}

Here 1 - 4 denote the three parameter setups (see Table 3.2) of $P E_{1,2}$ and the one only with physical springs (without $\left.P E_{1,2}\right) . f c_{m, i}(m=1,2, \ldots, 5,6$, see Eq.(3.23)) is a normalized impact force at a leg with the setup $i(i=1,2,3,4)$.

One can see that AMOS's legs suffer less impact forces (see $F C_{1-3}$ in Fig. 3.15) when its joints are driven by the pairs of $P E_{1,2}$, compared with legs only having passive springs (see $F C_{4}$ in Fig. 3.15). This is because AMOS can automatically adapt its height (see Figs. 3.16 (a), (b), and (c)) when its CTr and FTi joints are driven by the pairs of $P E_{1,2}$ (see Fig. 3.13). Among the three setups of $P E_{1,2}$ (see Table 3.2), the setup $\mathrm{S} 2$ (i.e., $K=0.8, D=0.01$ ) results in less impact force (see $F C_{2}$ in Fig. 3.15), compared to other two setups (see $F C_{(1,3)}$ in Fig. 3.15). This is because the setup S2 allows AMOS to produce compliant and stable joint motions, which adapt its body height equally to the height of the supporters (see Fig. 3.16 


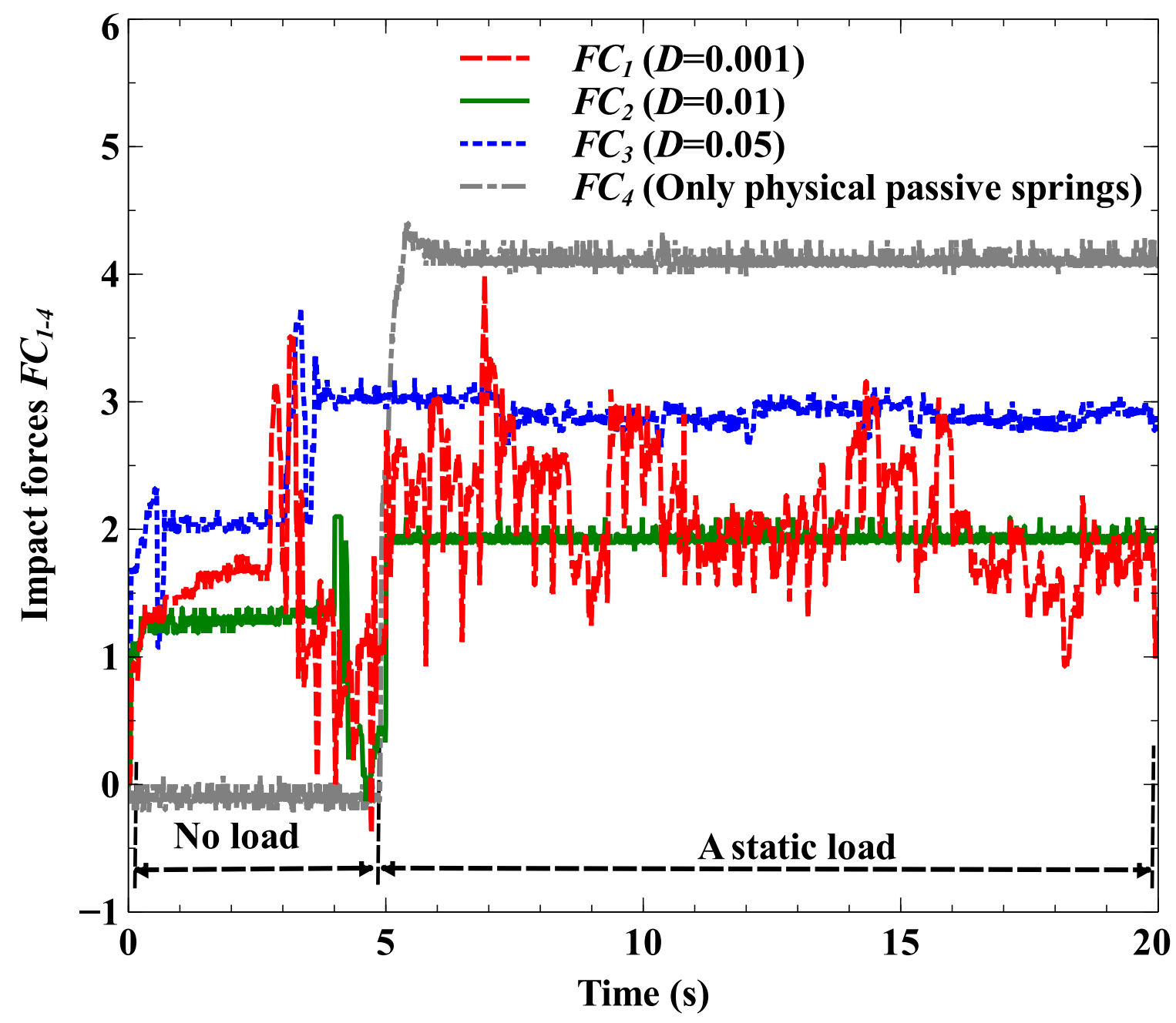

Figure 3.15: Impact forces $\mathrm{FC}_{1-4}$ arise from four experimental setups (see Figs. 3.16). We set the stiffness parameter $K$ in Eq.(3.17) to 0.8. After AMOS is imposed with the static load, its legs driven by $P E_{(1,2)}$ suffer less impact forces than without $P E_{(1,2)}$. Moreover, its legs suffer less impact forces when stiffness and damper parameters of $P E_{(1,2)}$ are set as: $[K, D]=[0.8,0.01]$.

(b)). This allows AMOS and the supporters to share the load of the board, which leads to less suffered impact force (see $F C_{2}$ in Fig. 3.15). By contrast, the setup S3 (see Table 3.2) results in stiffer (i.e., less compliant) joint motions pushing the body against the load (see Fig. 3.16 (c)), compared to the setup S2 (see Fig. 3.16 (b)). With the two setups, the suffered impact forces $f c_{m,(2,3)}$ at AMOS's legs are given by 
(see Figs. $3.16(\mathrm{~b})$ and (c)):

$$
f c_{m,(2,3)}=f c_{m} \cos \left(\theta_{(2,3)}\right), \theta 2>\theta 3, f c_{m, 2}<f c_{m, 3}, m=1,2, \ldots, 5,6
$$

Therefore, the setup $\mathrm{S} 2$ enables AMOS to suffer less impact force (i.e., $F C_{2}$, see Eq.(3.22)), compared to the setup S3 (see Table 3.2). On the other hand, the setup S1 (see Table 3.2) makes AMOS produce more compliant joint motions, which lead to unstable interactions against the static load.

(a)

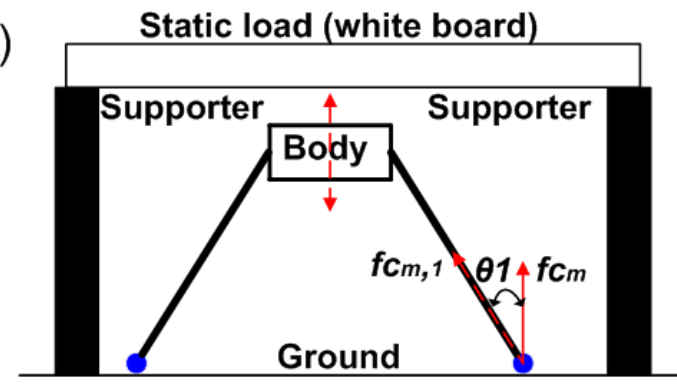

(c)

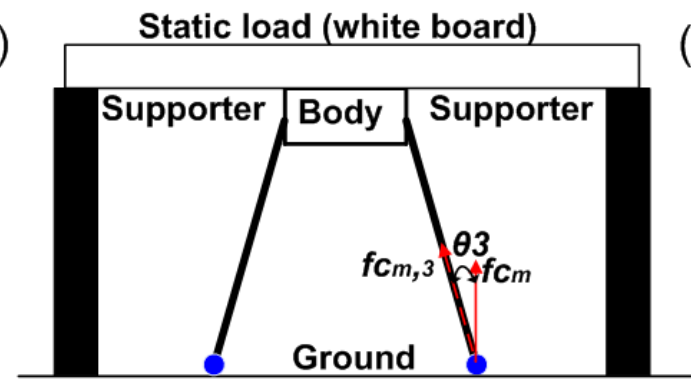

(b)

Static load (white board)

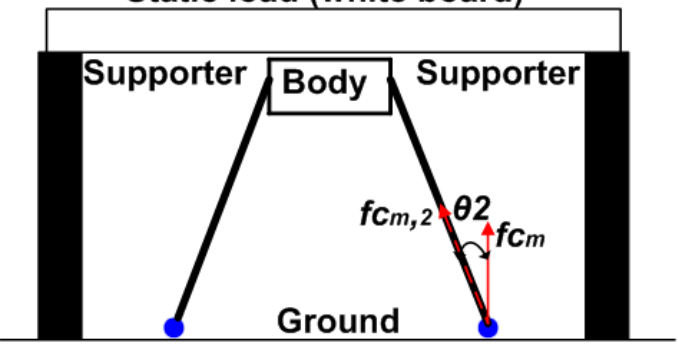

Static load (white board)

(d)

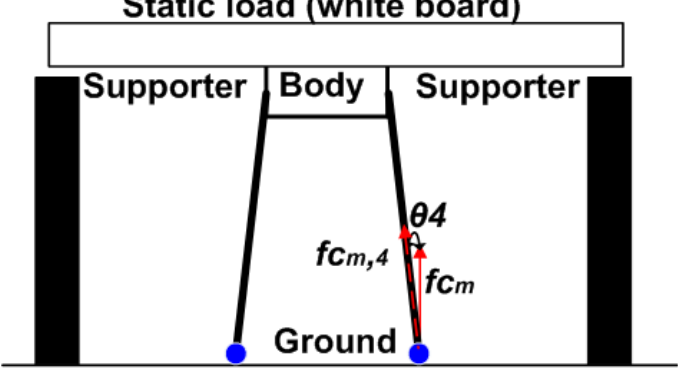

Figure 3.16: Schematic diagrams of the experiments perturbed with a static load. The muscle-like mechanisms (i.e., PEs of the VAAMs) enable the hexapod robot AMOS to produce variable compliant joint motions against a static load (i.e., white board), which leads to reducing the impact forces. This is because such compliant joint motions lower AMOS's body down such that AMOS and supporters share the weight of the static load. Whereas AMOS only with physical springs has to resist against the static load by its own. (a) $D=0.001$. (b) $D=0.01$. (c) $D=0.05$. (d) only with physical springs. The angles are: $\theta 1>\theta 2>\theta 3>\theta 4$.

In addition to reducing impact force, the three parameter setups (see Table 3.2) enable $P E_{(1,2)}$ to act muscle-like functions (e.g., the springs, see Figs. 3.17) that are comparable to those of biological muscles (Dickinson et al., 2000). This leads to soft robotic interactions against dynamic loads, which prevent AMOS from the damages (e.g., damaged legs). 


\subsubsection{Variable soft interactions against dynamic loads}

The experimental setup (see Fig. 3.13) of $P E_{(1,2)}$ of AMOS here is similar to that shown in Fig. 3.14. However, instead of a static load, dynamic loads were used here, e.g., hand pushing and releasing. Variably fighting against such loads results from variable compliant joint motions by empirically adjusting the damper parameter $D$ (see Eq.(3.21) and Table 3.2) of $P E_{(1,2)}$. The compliance of joint motions increases with the decreasing damper parameters. Here the stiffness parameter $K$ (see Eq.(3.20)) is fixed to 0.8. Interestingly, these three setups (see Table 3.2) enable $P E_{(1,2)}$ to generate muscle-like functions (see Figs. 3.17) that are comparable to those of biological muscles (Dickinson et al., 2000). Here the muscle-like functions are characterized by force-length loops of $P E_{(1,2)}$ of the VAAMs, in which the forces $f_{(1,2)}^{P}$ and lengths $l_{(1,2)}^{P}$ are given by Eqs.(3.15) and (3.16). The functions (e.g., springs) can be inferred by the shapes of their force-length loops. For example, one can see that the damper parameters $D=0.001$ and $D=0.01$ enable $P E_{(1,2)}$ to yield different and slanted straight force-length loops (see Figs. 3.17 (a) and (b)). Thus these two parameter setups make $P E_{(1,2)}$ act as the compliant springs when confronted with dynamic loads (i.e., hand pushing and releasing). However, the parameter setups enable $P E_{(1,2)}$ to produce more oscillations of shortening and lengthening, thereby leading to difficultly stabilizing AMOS's joints. By contrast, $P E_{(1,2)}$ become stiffer and easily stabilize the joints (see the initial point $O$ and the stabilization points $S_{(1,2)}$ in Fig. 3.17 (c)) when their $D$ values are set to 0.05 . This is because $P E_{(1,2)}$ act as the dampers (i.e., load absorbers) that only absorb energy from the load. All force-length loops presented in Figs. 3.17 are comparable to those of animal muscles (see Fig. 3 of Dickinson et al. (2000)). The springs and dampers mentioned in this thesis refer to the descriptions in Dickinson et al. (2000). The experimental video can be seen at https://www.youtube.com/watch?v=0c_HcitlXr8. We would like to also emphasize that the VAAMs are comprised of the contractile and parallel elements (i.e., $C E_{(1,2)}$ and $\left.P E_{(1,2)}\right)$. In Chapter 4, we will integrate the VAAMs with a modular neural network (MNN) where its neural outputs will serve as neural activities of $C E_{(1,2)}$ of the VAAMs. The integration (i.e., neuromechanical control) with proper stiffness parameters enables AMOS to produce insect-like and variable compliant walking, thereby leading to more energy-efficient walking on rough surfaces (e.g., gravel). Allowing the insect-like muscle functions (i.e., brakes, see Figs. 3.11 (c) and (d)), the proper stiffness parameters (e.g., $K=3.0$ or $K=4.0$ ) will be applied to $P E_{(1,2)}$ that control the distal joints (i.e., FTi joints) of AMOS under a proximo-distal gradient of neural control and muscle functions. 


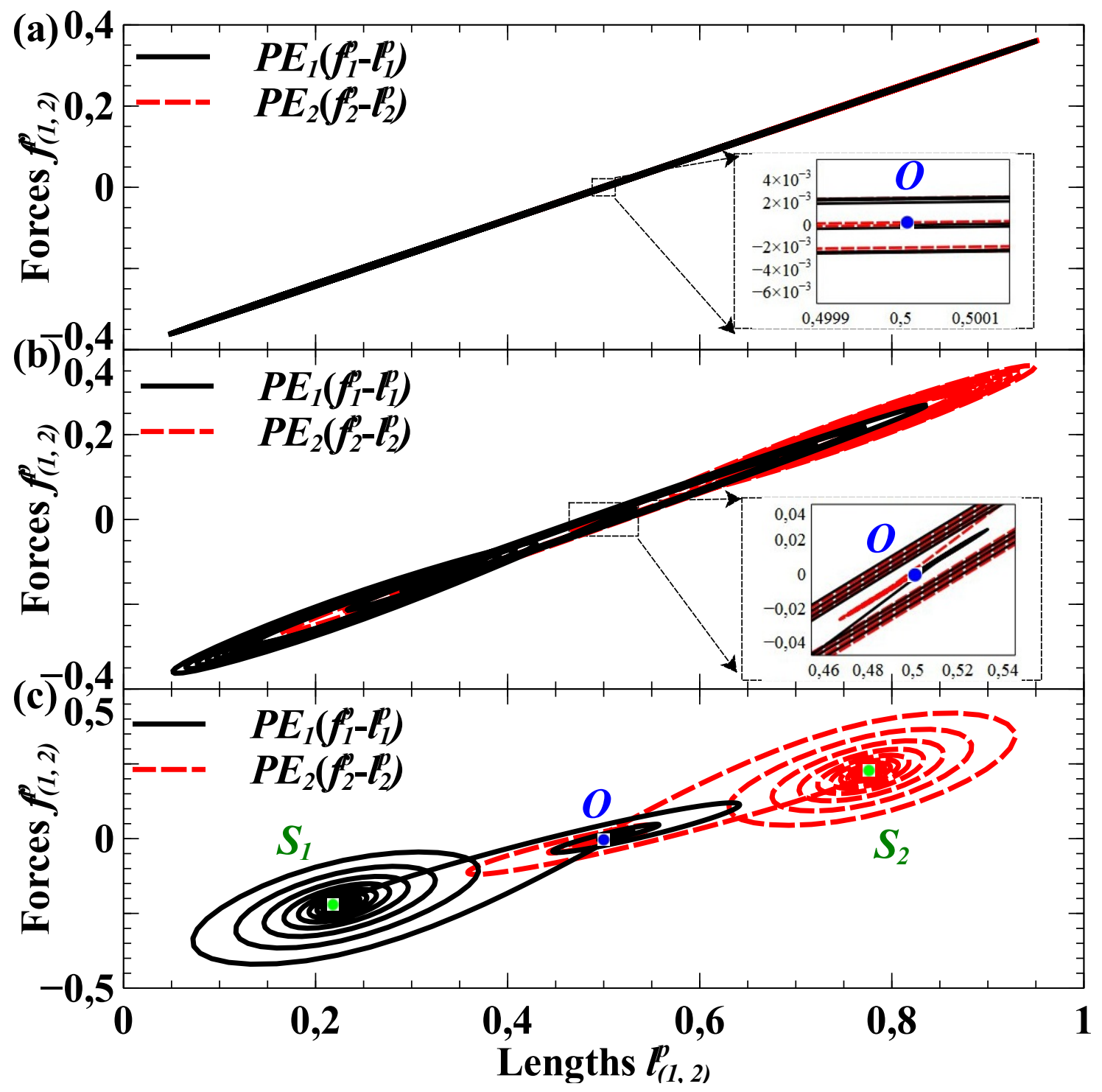

Figure 3.17: Muscle-like functions of $P E_{(1,2)}$ driving the FTi joint. The muscle-like function of $P E_{(1,2)}$ are comparable of those (i.e., springs and dampers) of biological muscles (Dickinson et al., 2000). The implementations rely only on changing the damper parameters $D$ (see $D$ values in Table 3.2) of $P E_{(1,2)}$ driving the FTi joint. (a) Springs $(D=0.001)$. (b) Springs $(D=0.01)$. (c) Dampers $(D=0.05)$. $O$ is the starting point of the force-length loops. Here we fix the stiffness parameter $K$ in Eq.(3.15) to 0.8. More details of the springs and dampers can be seen at the texts. 
54 Chapter 3: Biomechanical mechanisms for Variable Compliance Control 


\section{4 Neuromechanical Control for Variable Compliant Hexapedal Walking}

In Chapter 3, one can see that the virtual agonist-antagonist mechanisms (VAAMs) rely only on their passive elements (i.e., PEs) to generate insect-like muscle functions (i.e., the brakes, see Figs. 3.11 (c) and (d)). Such functions enable the driven joints to stably and compliantly fight against the perturbations using force feedback (i.e., see $f^{e x t}$ in Eq.(3.13)). The insect-like muscle functions are characterized by produced passive forces and lengths (see Eqs.(3.15) and (3.16)) of the PEs excited by force feedback $f^{e x t}$. In addition to feedback control, the VAAMs generate active forces (see Eq.(3.5)) deriving from feed-forward neural control (i.e., the modular neural network, see Figs. 4.2). Integrating the two control ways (see Eq.(3.12)), the VAAMs can emulate the muscles driving the proximal and distal joints in a proximo-distal gradient (see Fig. 2.5) that enhances locomotor stability (Daley et al., 2007; Nishikawa et al., 2007; Lee and Biewener, 2011; Lee et al., 2008a). In such a gradient, the proximal joints are less sensitive to the loads and under feed-forward neural control. By contrast, the distal joints are more sensitive to the loads, and under feedback (e.g., force) control that allows variable compliant joint motions against the loads. Thus based on the gradient, the neuromechanical control of AMOS's three-jointed legs is as follows (see Fig. 4.1): its proximal joints (i.e., TC joints) are driven only by the CEs of the VAAMs (see (1) in Fig. 4.1), its intermediate joints (i.e., CTr joints) are driven by the CEs and PEs of the VAAMs, and its distal joints (i.e., FTi joints) are driven only by the PEs of the VAAMs (see (2) in Fig. 4.1). The experimental results show that such a setup enables AMOS to achieve more stable walking on rough surfaces (see Fig. 4.5). Therefore the setup enhances stability of legged robot locomotion under classical active compliance control that generally leads to locomotor instabilities (Garcia and de Santos, 2006; Fukuoka et al., 2003). With the setup AMOS achieves variable compliant joint motions that result from different stiffness parameters $K_{j}(j=7,8, \ldots, 17,18)$ of the PEs of the VAAMs, while their CEs are used to coordinate its joints by receiving the neural outputs $N_{i}(i=1,2, \ldots, 17,18)$.

As a result, its coordinated and variable compliant joint motions lead to more energy-efficient walking on rough surfaces (e.g., gravel). Moreover, the compliant 


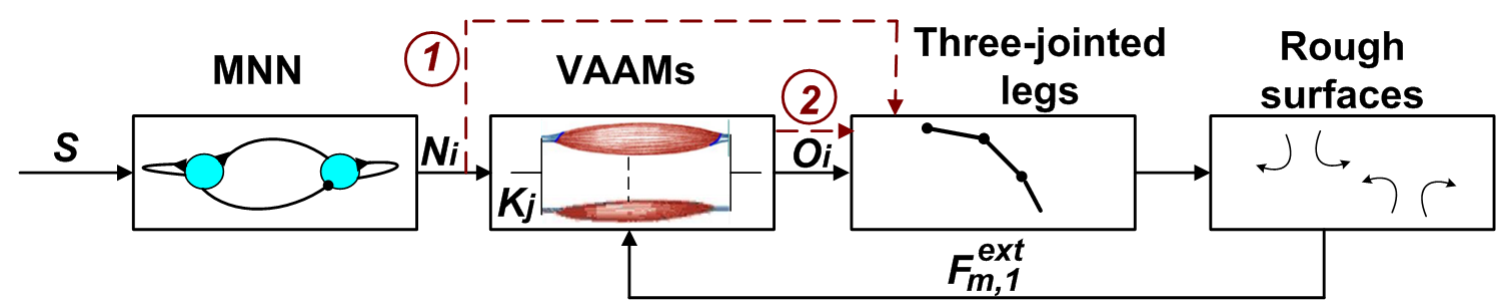

Figure 4.1: A neuromechanical controller coupled with the proximo-distal gradient. Based on the gradient, the proximal joints are driven only by the CEs of the virtual agonist-antagonist mechanisms (VAAMs) using the neural outputs $N_{i}$ $(i=1,2, \ldots, 17,18)$ of the modular neural network (MNN) (see (1). By contrast, the distal joints are driven only by the PEs of the VAAMs under force feedback (i.e., $\left.F_{m, 1}^{e x t}, m=1,2, \ldots, 5,6\right)$ control (see (2)). Based on the same feedback, the intermediate joints are controlled by the CEs and PEs of the VAAMs via descending commands (i.e., $S, N_{i}$, and $O_{i}$ ). Such a setup enables AMOS to perform stable and variable compliant walking on rough surfaces (e.g., gravel). The variable compliant walking results from changing the stiffness parameters $K_{j}(j=7,8, \ldots, 17,18)$ of the PEs of the VAAMs.

joint motions are utilized to well classify the surfaces. Such compliant motions are generated by manually choosing the proper stiffness and damper parameters (i.e., $K_{j}$ and $\left.D_{j}, j=7,8, \ldots, 17,18\right)$ of the PEs driving the CTr and FTi joints. At the FTi joints, for example, the stiffness parameters $K_{(13-18)}=3.0$ or 4.0 enable the PEs to act like insect-like muscle functions (i.e., the brakes (Ahn and Full, 2002), see Figs. 3.11 (c) and (d)). Moreover, their damper parameters $D_{(13-18)}$ are properly fixed to 1.0 such that the FTi joints can stably and compliantly fight against the perturbed loads (see Figs. 3.9). In addition, a proper insect-like gait of AMOS will be generated by fixing the neural input $S$ of the modular neural network (MNN).

\subsection{The modular neural network}

The modular neural network (MNN) is a biologically-inspired hierarchical neural controller (McCrea and Rybak, 2008), which coordinates the biomechanical components consisting of the three-jointed legs (see Fig. 3.3 (b)) and the virtual agonist-antagonist mechanisms (i.e., VAAMs, see Figs. 3.4). In such coordination, the outputs of the MNN serve as the neural activities of the CEs of the VAAMs coordinating the 18 joints of the hexapod robot AMOS. The MNN consists of a central pattern generator (CPG, see Fig. 4.2 (a)), a phase switch module (PSM, see Fig. 4.2 (b)), and two velocity regulating modules (VRMs, see Fig. 4.2 (c)). All neurons of the MNN are modeled as discrete-time, non-spiking neurons. The activation $H_{i}$ of each neuron 


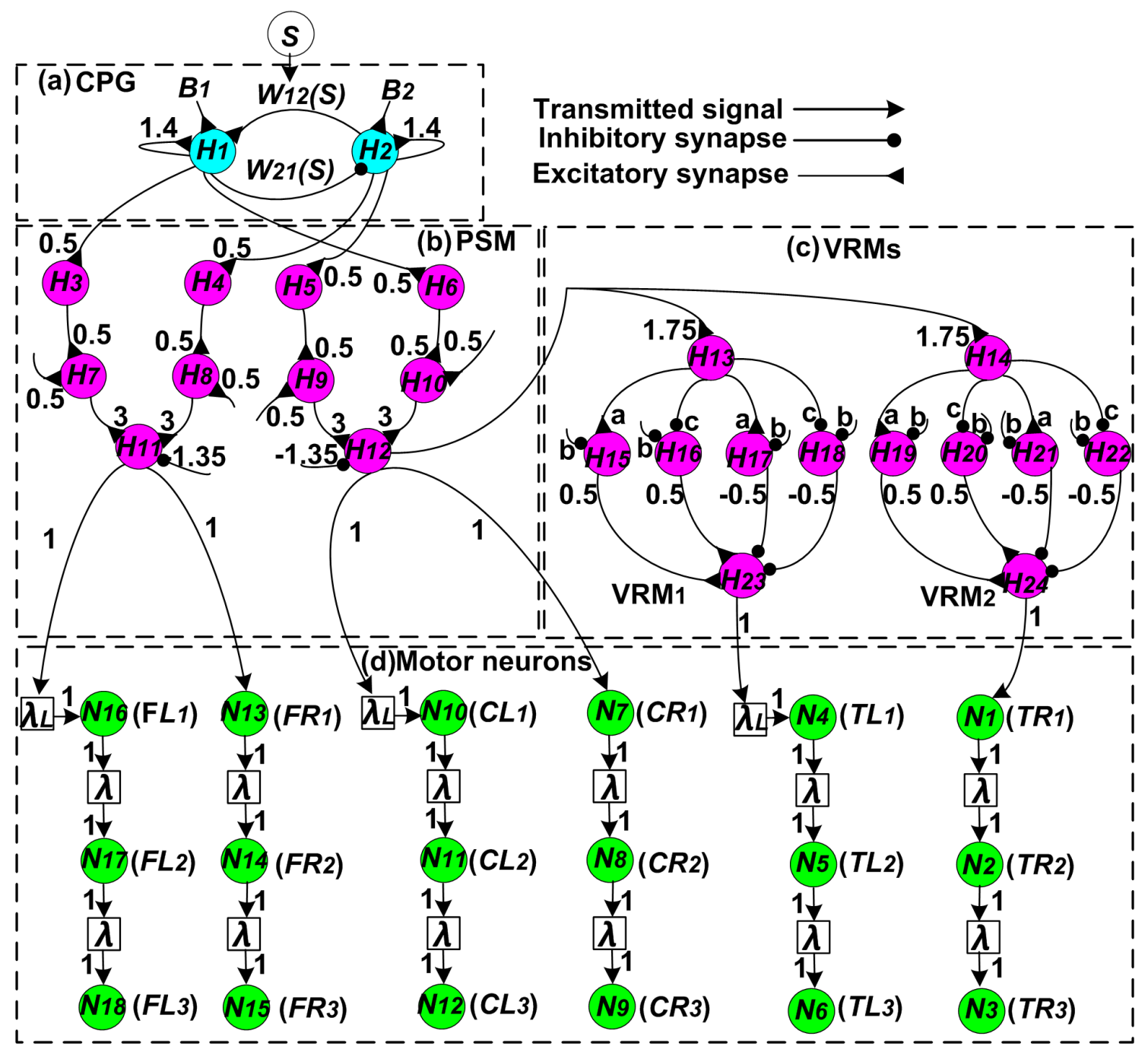

Figure 4.2: Modular neural network. There are three different neuron groups: input neuron $(S)$, hidden neurons $\left(H_{1-24}\right)$ and output neurons $\left(N_{1-18}\right)$. The input neuron is used to control the gaits of the hexapod robot AMOS. The hidden neurons are divided into three modules: CPG, PSM and VRMs, which have different functionalities (see text for details). The output neurons represent the neural activities of the CEs of the VAAMs (see Figs. 3.4) driving the TC, CTr, and FTi joints. All connection strengths together with bias terms are indicated by the small numbers except some parameters of the VRMs $(a=1.7246, b=-2.48285, c=-1.7246)$. Delays $\lambda_{L}$ and $\lambda$ between output neurons are set to 48 time steps and 16 time steps, respectively. The abbreviations are: $T R(L)_{1,2,3}=$ TC joints of the Right(Left) Front, Middle, Hind legs, $C R(L)_{1,2,3}=\mathrm{CTr}$ joints of the Right(Left) Front, Middle, Hind legs, $F R(L)_{1,2,3}$ $=$ FTi joints of the Right(Left) Front, Middle, Hind legs. 
develops according to:

$$
H_{i}(t)=\sum_{j=1}^{m} W_{i j} o_{j}(t-1)+B_{i}, \quad i=1, \ldots, m,
$$

where $m$ denotes the number of units, $B_{i}$ is an internal bias term (i.e., stationary input) to neuron $i$, and $W_{i j}$ is the synaptic strength of the connection from neuron $j$ to neuron $i$. The output $o_{i}$ of all neurons of the MNN is calculated using a hyperbolic

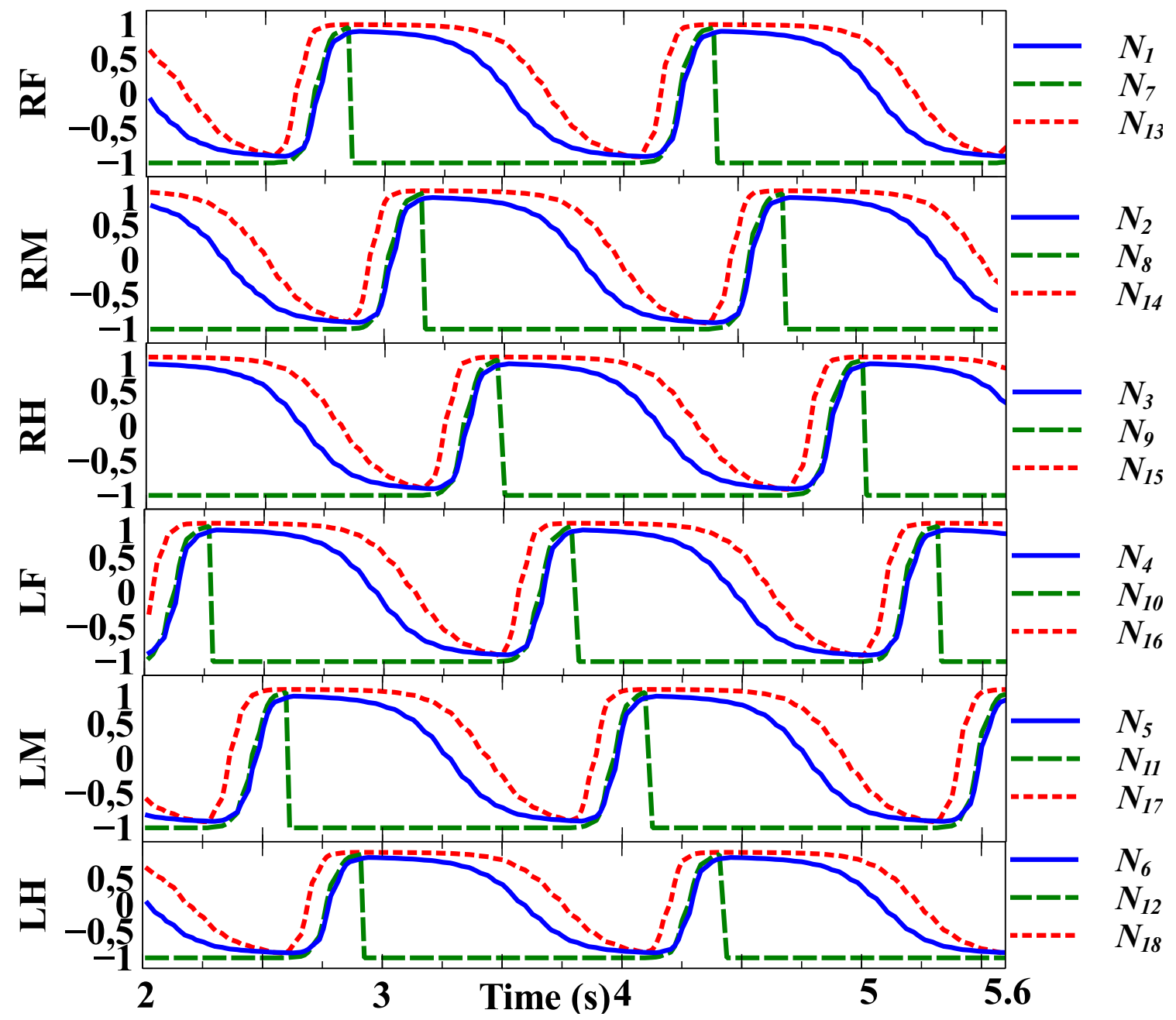

Figure 4.3: Outputs of the motor neurons $N_{1-18}$. Here the modulatory input $S$ of the modular neural network is set to 0.04. This results in a slow walking behavior (i.e., a slow wave gait, see Fig. 5.1 (b)). Abbreviations are: R(F, M, H) = Right (Front, Middle, Hind) leg, L(F, M, H) = Left (Front, Middle, Hind) leg.

tangent (tanh) transfer function, i.e., $o_{i}=\tanh \left(H_{i}\right) \in[-1,1]$. The weights $W_{i j}$ are 
manually designed, except weights $a, b$, and $c$ that are obtained by back-propagation learning (see Fig.4.2 (c)). For more details of determining the weights $W_{i j}$, we refer to the previous work (Manoonpong et al., 2008) of the MNN. The CPG consists of only two neurons with full connectivity (see Fig. 4.2(a)), where $B_{1}$ and $B_{2}$ are set to 0.01 . The weights $W_{12}$ and $W_{21}$ are given by:

$$
W_{12}(S)=0.18+S, W_{21}(S)=-0.18-S,
$$

where $S \in[0.01,0.18]$ is the modulatory input of the MNN, which determines the speed of AMOS's legs, which increases with increasing $S$. Here, we set $S$ to 0.04 resulting in a slow wave gait (see Figs.4.3 and 5.1 (b)) of AMOS, which leads to its stable and energy-efficient locomotion on uneven surfaces (Manoonpong et al., 2013b). The PSM is a generic feed-forward network consisting of three hierarchical layers with ten hidden neurons (i.e., $H_{3}-H_{12}$ ) (see Fig. $4.2(\mathrm{~b})$ ). The outputs of the PSM are projected to the FTi (i.e., $\left.F(R, L)_{(1,2,3)}\right)$ and $\mathrm{CTr}$ (i.e., $\left.C(R, L)_{(1,2,3)}\right)$ motor neurons (see Fig. $4.2(\mathrm{~d})$ ), as well as to the neurons $H_{13}$ and $H_{14}$ of the two VRMs (see Fig. $4.2(\mathrm{c})$ ). The VRMs are feed-forward networks projecting their outputs to the TC motor neurons $T(R, L)_{(1,2,3)}$ (see Fig. $\left.4.2(\mathrm{~d})\right)$. Delays $\lambda_{L}$ and $\lambda$ between the motor neurons are fixed (see Fig. $4.2(\mathrm{~d})$ ). For more details of the MNN, we refer to the previous work (Manoonpong et al., 2013b).

\subsection{The neuromechanical principle}

The neuromechanical principle (i.e., the proximo-distal gradient, see Fig. 4.1) is applied to neuromechanical control of the hexapod robot AMOS, thereby leading to its stable and variable compliant walking on the rough surfaces (e.g., gravel). The neuromechanical controller consists of the neural network (i.e., MNN, see Figs. 4.2) and several muscle-like mechanisms (i.e., VAAMs, see Figs. 3.4). The outputs $O_{1-18} \in$ $[-1,1]$ of the controller are linearly scaled and transmitted to control the positions of the standard servo motors driving the 18 joints of AMOS (see Figs. 4.4). Note that the command $O_{0}$ here is set to a constant value (i.e., $O_{0}=0$ ) for controlling the backbone joint to the middle position. For joint control (i.e., $O_{1-18}$ ), different control strategies are applied to stance and swing phases, like virtual model controllers (Pratt et al., 2001; Hutter et al., 2011).

\subsubsection{Stance phase}

According to the three-jointed leg of AMOS, the TC joint of the leg allowing only horizontal motion is not affected by the PEs of the VAAM since there is only detection of vertical foot force at the end effector of the leg. As a consequence, the TC joint is driven only by the CEs of the VAAM. By contrast, the CTr and FTi joints of the leg allowing vertical motions can be influenced by vertical foot force. The force 
(a)

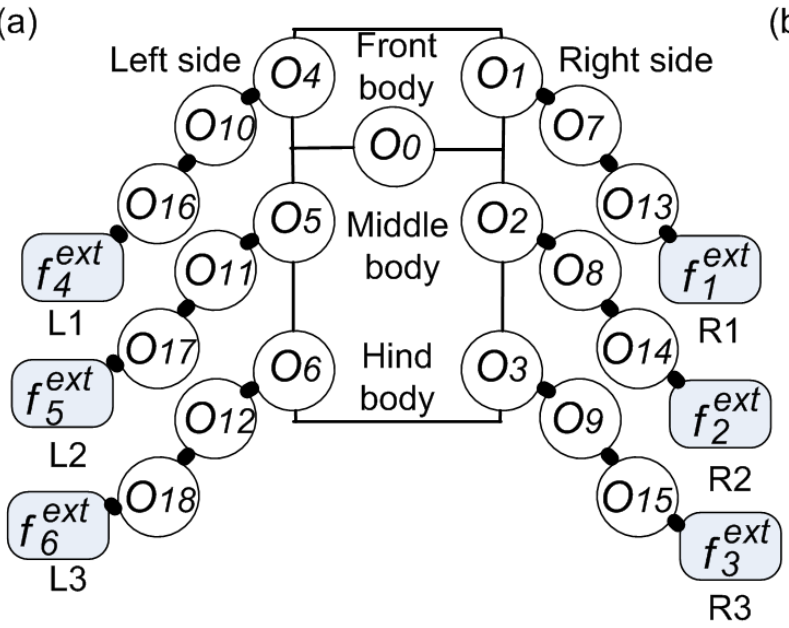

(b)

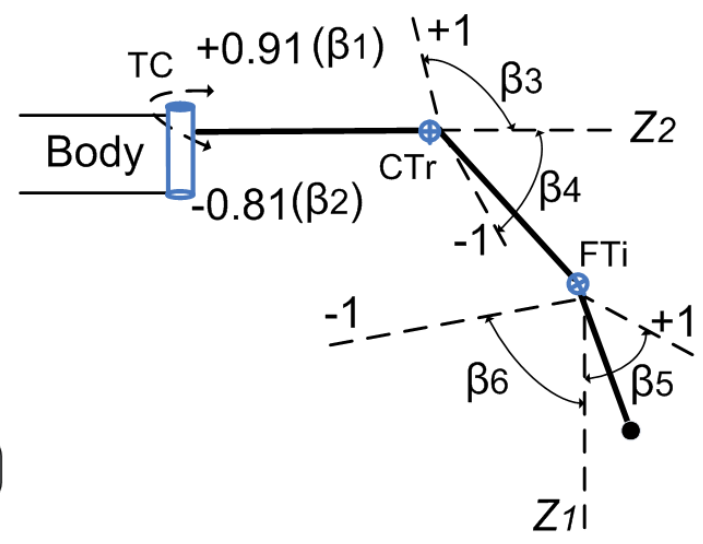

Figure 4.4: The outputs $O_{1-18}$ of the neuromechanical controller. (a) $O_{1-18}$ control the 18 joints of AMOS when receiving analog signals $f_{1-6}^{e x t}$, which are detected by the force sensors at the legs. (b) Relationship between the joint outputs $O_{1-18}$ and the joint angles $\theta_{1-18}$. The angular range of the TC, $\mathrm{CTr}$ and FTi joints is as follows: $\left[\beta_{1}, \beta_{2}\right]=[0.32,-0.37](\mathrm{rad}),\left[\beta_{3}, \beta_{4}\right]=[-1.745,0.785](\mathrm{rad})$, $\left[\beta_{5}, \beta_{6}\right]=[0.96,-1.222](\mathrm{rad})$.

Table 4.1: Nine tested setups of the PEs and CEs driving the CTr and FTi joints

\begin{tabular}{|l|c|c|c|}
\hline CTr & PEs and CEs & PEs & CEs \\
\hline PEs and CEs & S1 & S2 & S3 \\
\hline PEs & S4 & S5 & S6 \\
\hline CEs & S7 & S8 & S9 \\
\hline
\end{tabular}

therefore activates the PEs to generate variable compliant joint motions. We tested nine possible setups of the PEs and CEs (see Table 4.1) driving the CTr and FTi joints in a physical simulator (i.e., lpzrobots simulator (Der and Martius, 2012)). The results show that the setup S2 that is the combination of feed-forward neural and force feedback control at the CTr joint and only force feedback control at the FTi joint, is the best compared to other combinations (see S2 in Table 4.1). This combination allows the hexapod robot AMOS to achieve better coordinated movement and stable locomotion with smaller body oscillations (see Fig.4.5).

Therefore, the control strategy of each three-jointed leg of AMOS is as follows: Each TC joint (i.e., proximal joint) is controlled only by the CEs of the VAAM leading to feed-forward neural control (i.e., pure actuation), each $\mathrm{CTr}$ joint (i.e., intermediate joint) is governed by the CEs and PEs of the VAAM combining feed-forward neural and force feedback control (i.e., actuation and compliance), and each FTi joint (i.e., 
distal joint) is driven only by the PEs of the VAAM resulting in force feedback control (i.e., pure compliance) (see Figs. 4.6). This control strategy also complies with the findings revealed by biological studies on three-jointed leg locomotion (Lee et al., 2008a; Raibert et al., 2008). The biological studies show that the proximal joints of animal legs mainly act as actuation while their distal joints serve as compliance and their intermediate joints show the combination (see Fig. 2.5). The outputs $O_{1-18}$ of the proposed neuromechanical controller are calculated as follows:

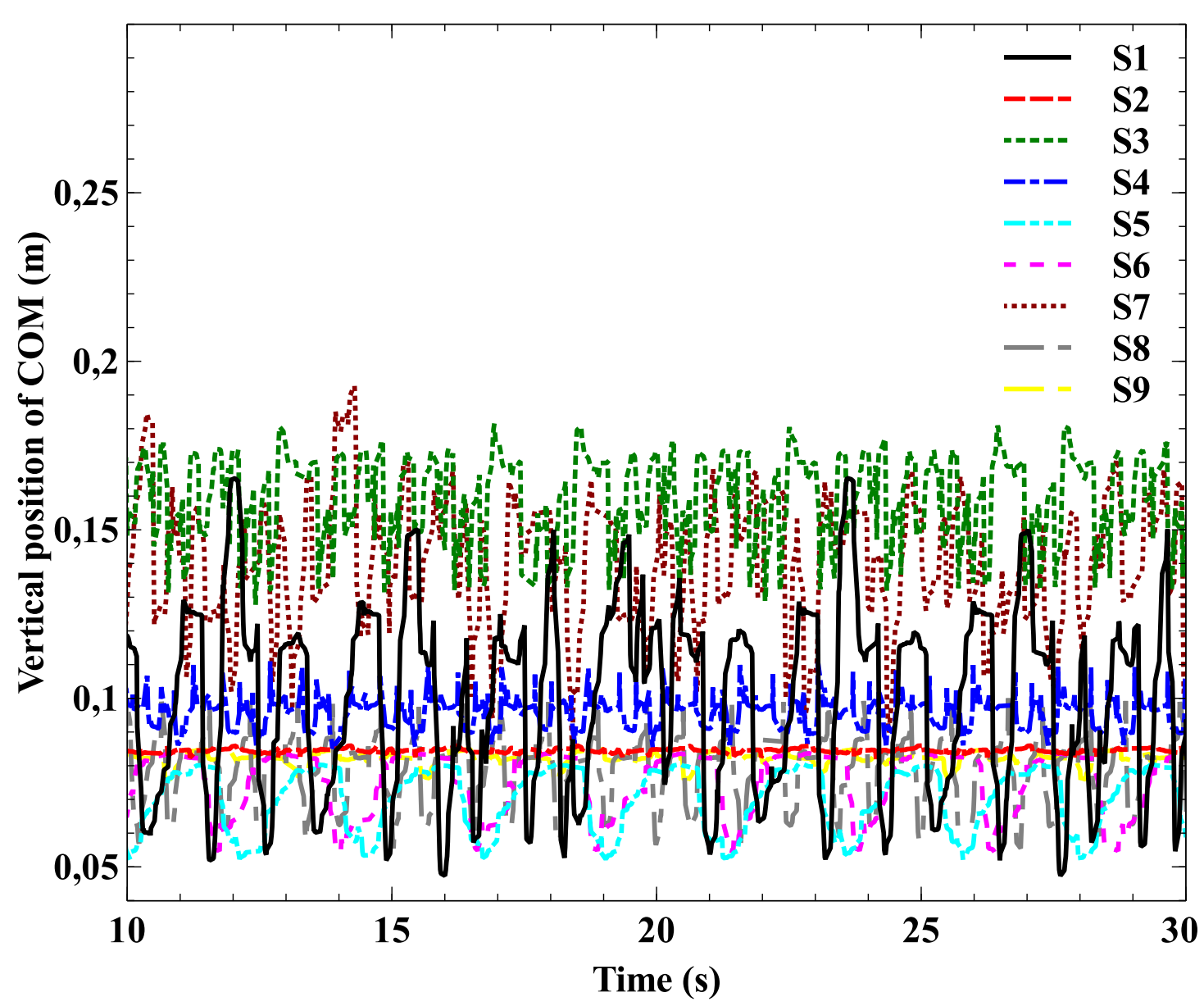

Figure 4.5: The vertical positions of the hexapod robot AMOS under nine setups of the PEs and CEs (see Table 4.1). The vertical positions show that AMOS walks stably (i.e., smaller body oscillations) when the FTi joints are controlled by only the PEs of the VAAMs, and the CTr joints are controlled by the CEs and PEs of the VAAMs (i.e., the setup S2). 
(a)

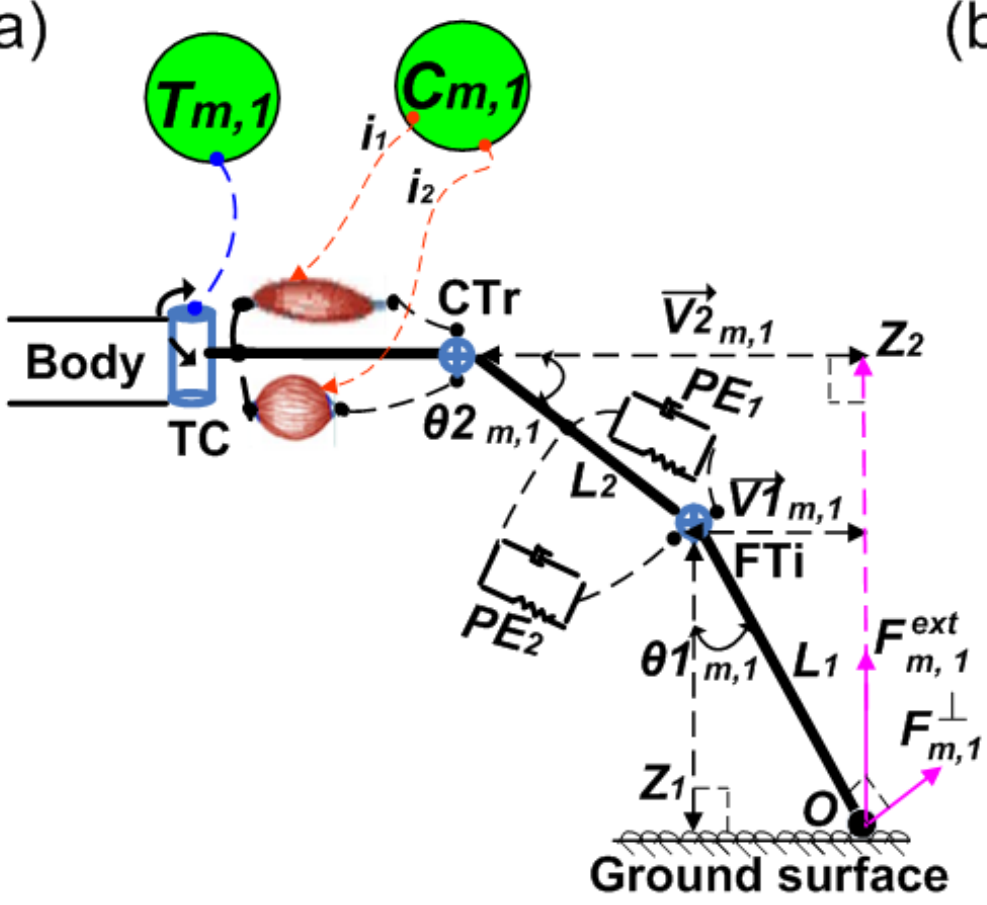

(b)

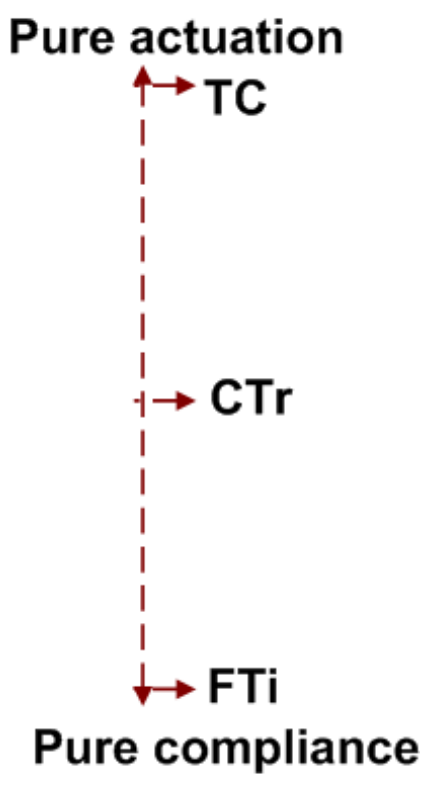

Figure 4.6: Neuromechanical control for each three-jointed leg. (a) The setup of the PEs and CEs driving the joints. The TC joint is controlled only by the output of the TC motor neuron $T_{m, 1}$ while the CTr joint is driven by the VAAM activated by the output of the CTr motor neuron $C_{m, 1}(m=1,2, \ldots, 5,6)$. All outputs of the motor neurons come from the MNN $\left(T_{1-6,1}=N_{1-6}\right.$ and $C_{1-6,1}=N_{7-12}$, see Fig. $\left.4.2(\mathrm{~d})\right)$. Besides, the FTi joint is driven only by a pair of parallel elements (i.e., $P E_{1}$ and $P E_{2}$ ) of the VAAM. (b) The roles of joint actuation and compliance. The roles refer to the findings revealed by biological studies on three-jointed leg locomotion (Lee et al., 2008a; Raibert et al., 2008) (see Fig. 2.5).

\section{FTi joints}

Each FTi joint is driven only by a pair of $P E_{(1,2)}$ of the VAAM (see Fig. 4.6 (a)). Therefore, the neural activations $N_{6 \times 1}$ to the VAAMs driving the FTi joints are set to zero,

$$
N_{6 \times 1}=[0,0, \ldots, 0]^{T}
$$

In addition, the forces $f_{1-6}^{e x t}$ directly result in the extensions and flexions of the FTi joints. Therefore, the matrix $\tau_{6 \times 1}^{F T i}$ of torques acting on the FTi joints is given by (derived from Eq.(3.10)):

$$
\tau_{6 \times 1}^{F T i}=\overrightarrow{V 1_{6 \times 1}}=F_{6 \times 1}^{\perp} L_{1}
$$


where

$$
F_{6 \times 1}^{\perp}=F_{6 \times 1}^{e x t} \circ \sin \left(\theta 1_{6 \times 1}\right)=\left[f_{1}^{\text {ext }} \sin \left(\theta_{13}\right), f_{2}^{\text {ext }} \sin \left(\theta_{14}\right), \ldots, f_{5}^{\text {ext }} \sin \left(\theta_{17}\right), f_{6}^{\text {ext }} \sin \left(\theta_{18}\right)\right]^{T}
$$

Derived from Eqs.(4.3), (4.4), and (3.12), the matrix $\theta 1_{6 \times 1}$ of the FTi joint angles is the sum of the Hadamard products:

$$
I \ddot{\theta} 1_{6 \times 1}=F_{6 \times 1}^{e x t} \circ \sin \left(\theta 1_{6 \times 1}\right) L_{1}-r\left(2 r K 1_{6 \times 1} \circ \theta 1_{6 \times 1}+2 r D 1_{6 \times 1} \circ \dot{\theta} 1_{6 \times 1}\right),
$$

where

$$
\begin{gathered}
\ddot{\theta} 1_{6 \times 1}=\left[\ddot{\theta}_{13}, \ddot{\theta}_{14}, \ldots, \ddot{\theta}_{17}, \ddot{\theta}_{18}\right]^{T}, \dot{\theta} 1_{6 \times 1}=\left[\dot{\theta}_{13}, \dot{\theta}_{14}, \ldots, \dot{\theta}_{17}, \dot{\theta}_{18}\right]^{T}, \\
K 1_{6 \times 1}=\left[K_{13}, K_{14}, \ldots, K_{17}, K_{18}\right]^{T}, D 1_{6 \times 1}=\left[D_{13}, D_{14}, \ldots, D_{17}, D_{18}\right]^{T} .
\end{gathered}
$$

The angles $\theta 1_{m, 1}(m=1,2, \ldots, 5,6)$ of the FTi joints can be linearly transformed into their outputs $O_{j}$ (see Fig. 4.4). $O_{j}$ are given by $(j=13,14, \ldots, 17,18)$ :

$$
O_{j}=0.92 \theta 1_{m, 1}+0.12, m=j-12 .
$$

The details of Eq.(4.6) can be seen at the appendix A.3.2 (i.e., see Eq.(A.12)).

\section{CTr joints}

Each $\mathrm{CTr}$ joint is driven by $P E_{(1,2)}$ and $C E_{(1,2)}$ of the VAAM. $C E_{(1,2)}$ are activated by one of the outputs $N_{7-12}$ (i.e., $C_{6 \times 1}=N_{7-12}$ ) of the MNN (see Fig. 4.6 (a)). For example, the pair of the VAAM driving the right front CTr joint is activated by $N_{7}$ of the MNN (see Fig. $4.2(\mathrm{~d})$ ). The forces $f_{1-6}^{\text {ext }}$ indirectly result in the elevations and depressions of the $\mathrm{CTr}$ joints. The matrix of the $\mathrm{CTr}$ joint angles is $\theta 2_{6 \times 1}=$ $\left[\theta_{7}, \theta_{8}, \ldots, \theta_{11}, \theta_{12}\right]^{T}$. The computation of the torques generated by $f_{1-6}^{e x t}$ needs to be approximated, since there are no torque sensors at the $\mathrm{CTr}$ joints. Therefore, the matrix $\tau_{6 \times 1}^{C T r}$ of the torques acting on the CTr joints is given by (derived from Eqs.(4.4) and (3.19)):

$$
\tau_{6 \times 1}^{C T r}=F_{6 \times 1}^{e x t} \circ \overrightarrow{V 2_{6 \times 1}}=F_{6 \times 1}^{e x t} \circ\left(L_{2} \cos \left(\theta 2_{6 \times 1}\right)+\overrightarrow{V 1_{6 \times 1}}\right),
$$

where

$\theta 2_{6 \times 1}=\left[\theta_{7}, \theta_{8}, \ldots, \theta_{11}, \theta_{12}\right]^{T}, \cos \left(\theta 2_{6 \times 1}\right)=\left[\cos \left(\theta_{7}\right), \cos \left(\theta_{8}\right), \ldots, \cos \left(\theta_{11}\right), \cos \left(\theta_{12}\right)\right]^{T}$

Derived from Eqs.(4.7) and (3.12), the matrix $\theta 2_{6 \times 1}$ of the CTr joint angles is the sum of the Hadamard products:

$$
I \ddot{\theta} 2_{6 \times 1}=\tau_{6 \times 1}^{C T r}+\left[r C_{6 \times 1}-2 r^{2}\left(K 2_{6 \times 1} \circ \theta 2_{6 \times 1}+D 2_{6 \times 1} \circ \dot{\theta} 2_{6 \times 1}\right)\right],
$$


where

$$
\begin{array}{r}
\ddot{\theta} 2_{6 \times 1}=\left[\ddot{\theta}_{7}, \ddot{\theta}_{8}, \ldots, \ddot{\theta}_{11}, \ddot{\theta}_{12}\right]^{T}, \dot{\theta} 2_{6 \times 1}=\left[\dot{\theta}_{7}, \dot{\theta}_{8}, \ldots, \dot{\theta}_{11}, \dot{\theta}_{12}\right]^{T}, \\
K 2_{6 \times 1}=\left[K_{7}, K_{8}, \ldots, K_{11}, K_{12}\right]^{T}, D 2_{6 \times 1}=\left[D_{7}, D_{8}, \ldots, D_{11}, D_{12}\right]^{T} .
\end{array}
$$

The angles $\theta 2_{m, 1}(m=1,2, \ldots, 5,6)$ of the $\mathrm{CTr}$ joints are linearly transformed into their outputs $O_{j}$ (see Fig. 4.4). $O_{j}$ are given by $(j=7,8, \ldots, 11,12)$ :

$$
O_{j}=-0.8 \theta 2_{m, 1}-0.38, m=j-6 .
$$

The details of Eq.(4.9) can be seen at the appendix A.3.2 (i.e., see Eq.(A.11)).

\section{TC joints}

Each TC joint is controlled only by a pair of $C E_{(1,2)}$ of the VAAM (see Fig. 4.6 (a)). The neural outputs (i.e., $N_{1-6}$ ) of the MNN serve as the neural activities of $C E_{(1,2)}$ driving the TC joints (i.e., $\left.T_{6 \times 1}=N_{1-6}\right) . T_{6 \times 1}$ are linearly transformed into the outputs $O_{j}$ controlling the TC joints $(j=1,2, \ldots, 5,6)$ :

$$
O_{j}=0.4 T_{j, 1}-0.05
$$

The details of Eq.(4.10) can be seen at the appendix A.3.2 (i.e., see Eq.(A.10)).

\subsubsection{Swing phases}

When the legs are in swing phases (i.e., $\left.f_{i}^{e x t}=0, i=1,2, \ldots, 5,6\right)$ ), their joints are controlled only by the CEs of the VAAMs receiving the neural outputs (i.e., $N_{1-18}$ ) of the MNN. $N_{1-18}$ are linearly transformed into the outputs $O_{1-18}$ controlling the joints $(i=1,2, \ldots, 5,6)$ :

$$
\left[O_{i}, O_{i+6}, O_{i+12}\right]^{T}=\left[0.4 N_{i}, 0.15 N_{i+6},-0.02 N_{i+12}\right]^{T}-[0.05,-0.86,0.43]^{T} .
$$

The details of Eq.(4.11) can be seen at the appendix A.3.1 (i.e., see Eqs.(A.7), (A.8), and (A.9)). Note that the last values of the outputs $O_{(i, i+6, i+12)}$ of the swing phase are kept and transferred to the initial joint angles of the following stance phase. This leads to smooth switches from swing to stance phases regardless of initial values of joint angles (see Fig. 4.7). On the other hand, the switches from stance to swing phases are determined by self-adjusting the proper stiffness parameters $K 1_{6 \times 1}$ and $K 2_{6 \times 1}$ (e.g., see Fig. 5.7). 


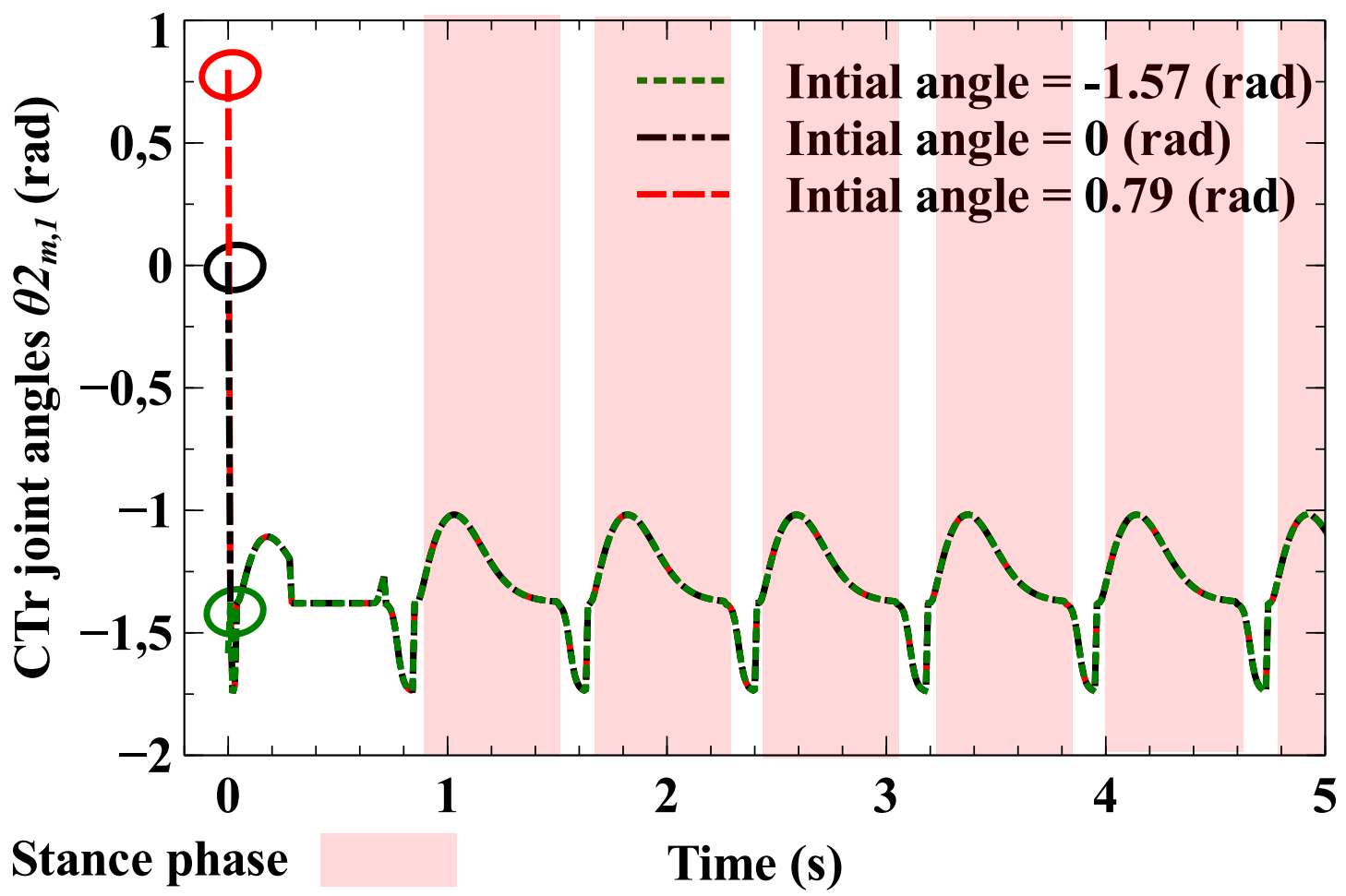

Figure 4.7: CTr joint angles $\theta 2_{m, 1}$ with respect to the initial joint angles. One can see that the same and smooth $\mathrm{CTr}$ joint angles $\theta 2_{m, 1}$ are generated even changing the initial joint angle. Here the stiffness and damper parameters are set to: $K 2_{m, 1}=9.0$ and $D 2_{m, 1}=1.0$.

\subsection{Stable and variable compliant walking on five surfaces}

The neuromechanical controller and principle (see Figs. 4.6) enable the hexapod robot AMOS to produce stable and variable compliant joint motions. The variable compliant motions are generated by changing the stiffness parameters (i.e., $K 1_{6 \times 1}$ in Eq.(4.5) and $K 2_{6 \times 1}$ in Eq.(4.8)) of the PEs driving the FTi and CTr joints. We tested 2601 setups of $K 1_{6 \times 1}$ and $K 2_{6 \times 1}$ for AMOS in the lpzrobots simulator (Der and Martius, 2012). By using these setups of $K 1_{6 \times 1}$ and $K 2_{6 \times 1}$, the forward displacements $X$ of the robot are used to measure the performance of its walking (see $X$ in Fig. 4.8). With each setup, the running time of its walking is $30 \mathrm{~s}$. One can see that AMOS performs proper walking behavior on a rough surface when $K(1,2)_{6 \times 1}$ are set within the range surrounded by black dash lines shown in Fig. 4.8. The result also certifies the Bigdog-inspired control strategy (Lee et al., 2008a; Raibert et al., 2008) (see Fig. 2.5). That is, the distal joints (e.g., FTi joints) should act more compliantly 


\section{Forward displacement $X$}

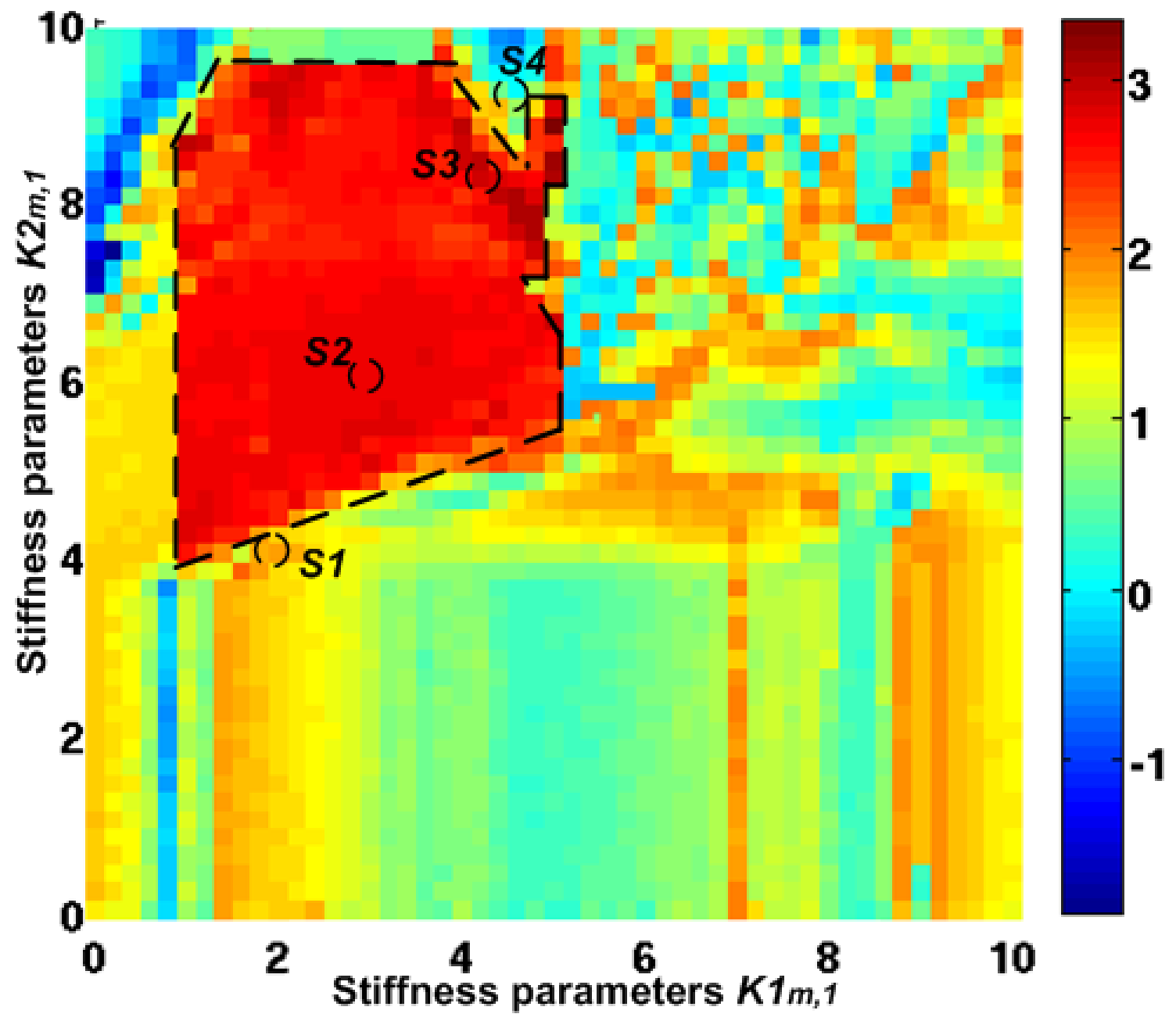

Figure 4.8: Forward displacements $X$ (unit: $m$ ) according to various setups of the stiffness parameters $K 1_{m, 1}$ and $K 2_{m, 1}$. Four setups (i.e., $S(1-4)$ ) of the stiffness parameters $K(1,2)_{6 \times 1}$ are chosen to test on our physical robot for walking over rough surfaces (e.g., gravel).

than the intermediate joints (e.g., CTr joints). Therefore, the stiffness parameters $K(1,2)_{m, 1}$ should be set as: $K 1_{m, 1}<K 2_{m, 1}$ (e.g., $2 \times K 1_{m, 1}=K 2_{m, 1}$ ). Four setups of $K(1,2)_{m, 1}$ are chosen for testing on AMOS to walk on physical rough surfaces (see $S(1-4)$ in Fig. 4.8):

$S 1: K 1_{m, 1}=2, K 2_{m, 1}=4$;

$S 2: K 1_{m, 1}=3, K 2_{m, 1}=6$; 
S3: $K 1_{m, 1}=4, K 2_{m, 1}=8$;

$S 4: K 1_{m, 1}=4.5, K 2_{m, 1}=9$.

Note that all damper parameters $D(1,2)_{m, 1}(m=1,2, \ldots, 5,6)$ of the PEs of the VAAMs driving the CTr and FTi joints are set to 1.0. This is because $D(1,2)_{m, 1}=1.0$ enable the PEs of the VAAMs to stably and compliantly fight against the perturbations (see Figs. 3.9). As a result, $S(1-4)$ make the PEs of the VAAMs driving the FTi joints generate the insect-like muscle functions (i.e., brakes, see Figs. 3.11 (c) and (d)) (Dickinson et al., 2000; Ahn and Full, 2002).

We tested the four setups (i.e., $S(1-4)$ ) of $K(1,2)_{6 \times 1}$ on AMOS to walk on coarse gravel $(\varphi=16-25 \mathrm{~mm})$, very coarse gravel $(\varphi=40-60 \mathrm{~mm})$, floor, snow (thickness $8 \mathrm{~cm}$ ), and sponge (stiffness $0.523 \mathrm{kN} / \mathrm{m}$ ) surfaces. AMOS, however, had a difficulty to walk on these surfaces when the lower stiffness setup (i.e., $S 1$ ) was used. This is because with this setup AMOS's legs shallowly penetrate into the surfaces (e.g., see $S 1$ in Fig. 4.9 (a)), thereby gaining less foot contact force (e.g., see $S 1$ in Fig. 4.9 (b)). By contrast, when the higher stiffness setup (i.e., S4) was used, its legs penetrate into the surfaces deeper (e.g., see $S 4$ in Fig. 4.9 (a)). However, this always causes unstable locomotion that can be observed from dropping of the foot contact force (e.g., see A1 of $S 4$ in Fig. 4.9 (b)). Therefore, with the high stiffness steup $S 4$ AMOS also had a difficulty to overcome all these surfaces. Note that "high stiffness" (i.e., high impedance) here means that a joint greatly resists the influence of external forces, thereby leading to low joint compliance (Pratt, 2000, 2002). By contrast, "low stiffness" (i.e., low impedance) here means that a joint allows external forces to influence its movement easily, thereby resulting in high joint compliance (Pratt, 2002; Pratt et al., 2002). Therefore, the stiffness of AMOS's legs increases with the increasing values of $K(1,2)_{6 \times 1}$. In contrast to the setups $S 1$ and $S 4$, the setup $S 2$ or $S 3$ allows the hexapod robot AMOS to stably walk on the surfaces. However, they lead to different energy efficiencies of its walking on the different surfaces. Energy efficiency is measured by cost of transport COT (i.e., specific resistance (Gregorio et al., 1997; Saranli et al., 2001)) as:

$$
C O T=\frac{P}{m g v},
$$

where $P$ is power consumption. $m g$ is the weight of the hexapod robot, i.e., $m g=$ 52.974 N. $v$ is its forward speed. For each stiffness setup (i.e., $S 2$ or $S 3$ ), we ran the hexapod robot at each surface ten times. For each run, the power consumption $P$ and average speed $v_{\text {avg }}$ were obtained. $C O T_{\text {avg }}$ with their standard deviations are shown in Table 4.2. Low $C O T_{\text {avg }}$ corresponds to more energy-efficient walking.

One can see that the high stiffness setup $S 3$ allows more energy-efficient locomotion (see Table 4.2) on loose surfaces (i.e., coarse gravel or snow). This is because the setup $S 3$ enables AMOS to stiffen its joints and legs, thereby obtaining more foot contact force (e.g., see Fig. 4.10 (c)) on these surfaces. By contrast, the low stiffness 


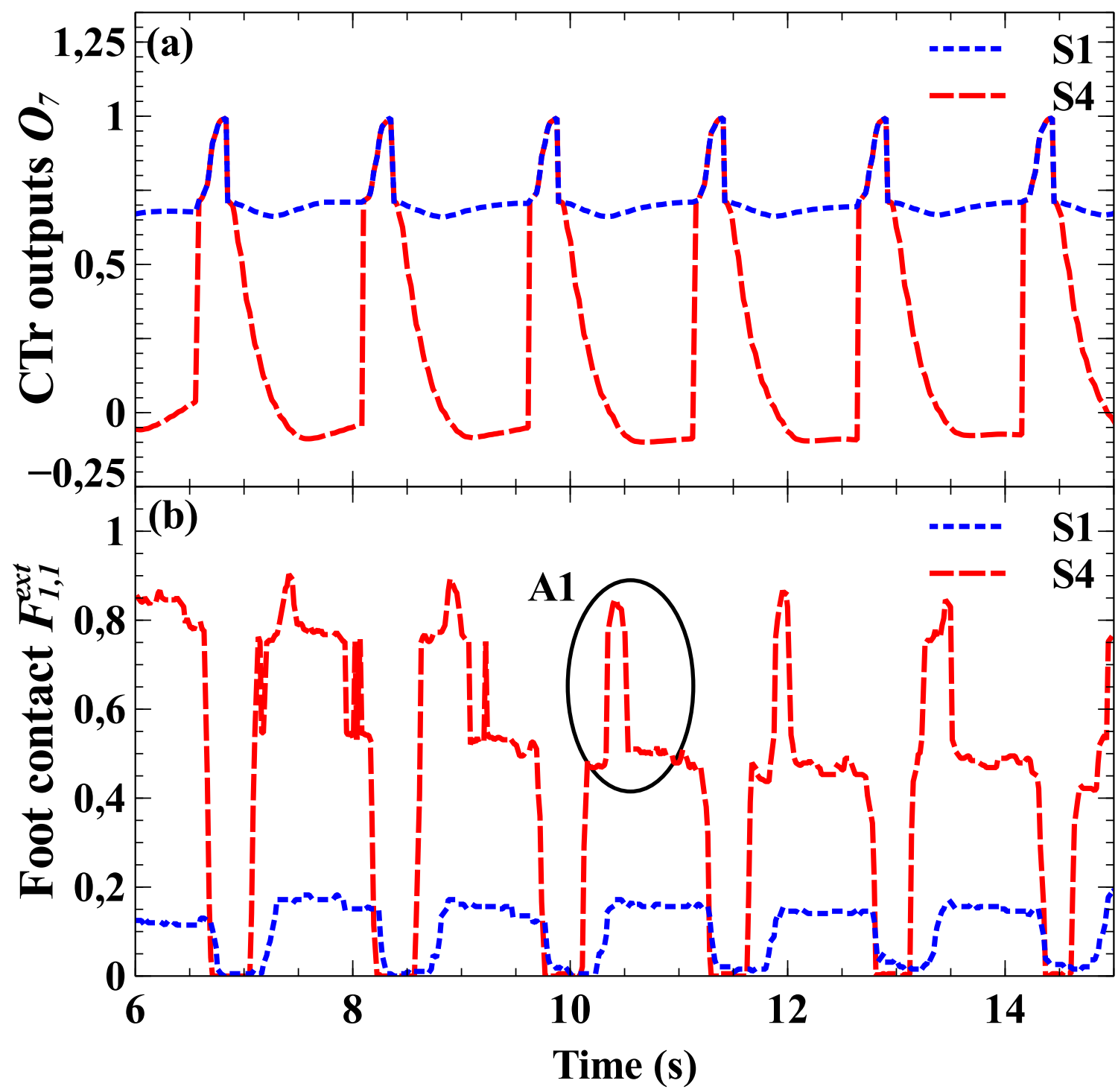

Figure 4.9: The CTr joint outputs and analog signals of foot force with stiffness setups $S 1$ and $S 4$. The signals were sensed when the hexapod robot walked on sponge surfaces. (a) CTr joint outputs $O_{7}$. (b) Analog signals of forces $F_{1,1}^{e x t}$.

setup $S 2$ results in more foot contact force (e.g., see Fig. 4.11 (c)) on flat (e.g., floor), very coarse gravel, and elastic (e.g., sponge) surfaces. Therefore, the setup $S 2$ leads to more energy-efficient locomotion on these surfaces. Interestingly, this experimental result shows that the softer legs allow an insect-like robot AMOS to achieve more energy-efficient locomotion on a soft elastic surface (e.g., sponge). The finding com- 
Table 4.2: Experimental statistics of $C O T_{a v g}$ with standard deviations

\begin{tabular}{|l|c|c|c|c|c|}
\hline \multirow{2}{*}{ Setups Surfaces } & $\begin{array}{c}\text { Coarse } \\
\text { gravel }\end{array}$ & $\begin{array}{c}\text { Very } \\
\text { coarse } \\
\text { gravel }\end{array}$ & Floor & Snow & Sponge \\
\hline \multirow{2}{*}{$S 3$} & $\mathbf{1 8 . 1} \pm$ & $25.0 \pm$ & $16.9 \pm$ & $\mathbf{1 8 . 8} \pm$ & $21.8 \pm$ \\
& $\mathbf{0 . 7}$ & 0.6 & 0.7 & $\mathbf{0 . 5}$ & 0.9 \\
\hline \multirow{2}{*}{$S 2$} & $20.7 \pm$ & $\mathbf{2 3 . 6} \pm$ & $\mathbf{1 4 . 0} \pm$ & $22.3 \pm$ & $\mathbf{1 9 . 7} \pm$ \\
& 1.1 & $\mathbf{0 . 5}$ & $\mathbf{0 . 4}$ & 0.8 & $\mathbf{0 . 8}$ \\
\hline
\end{tabular}

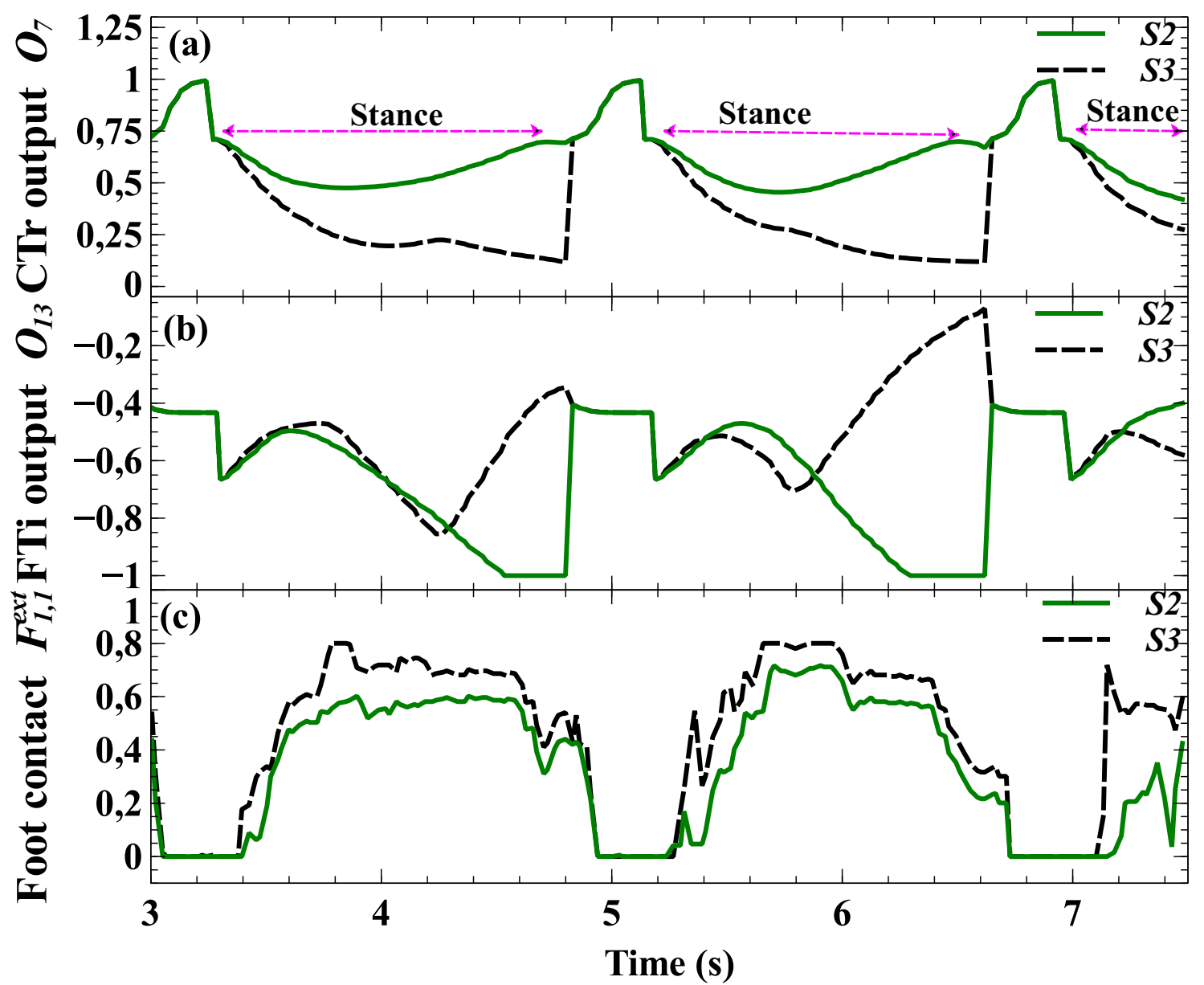

Figure 4.10: Joint outputs and foot force signals that resulted from two stiffness setups $S(2,3)$ during walking on coarse gravel. (a) CTr joint outputs $O_{7}$. (b) FTi joint outputs $O_{13}$. (c) Analog signals of forces $F_{1,1}^{e x t}$. The setup $S 3$ enables the hexapod robot to obtain more foot contact force during stance phases than the setup $S 2$. 


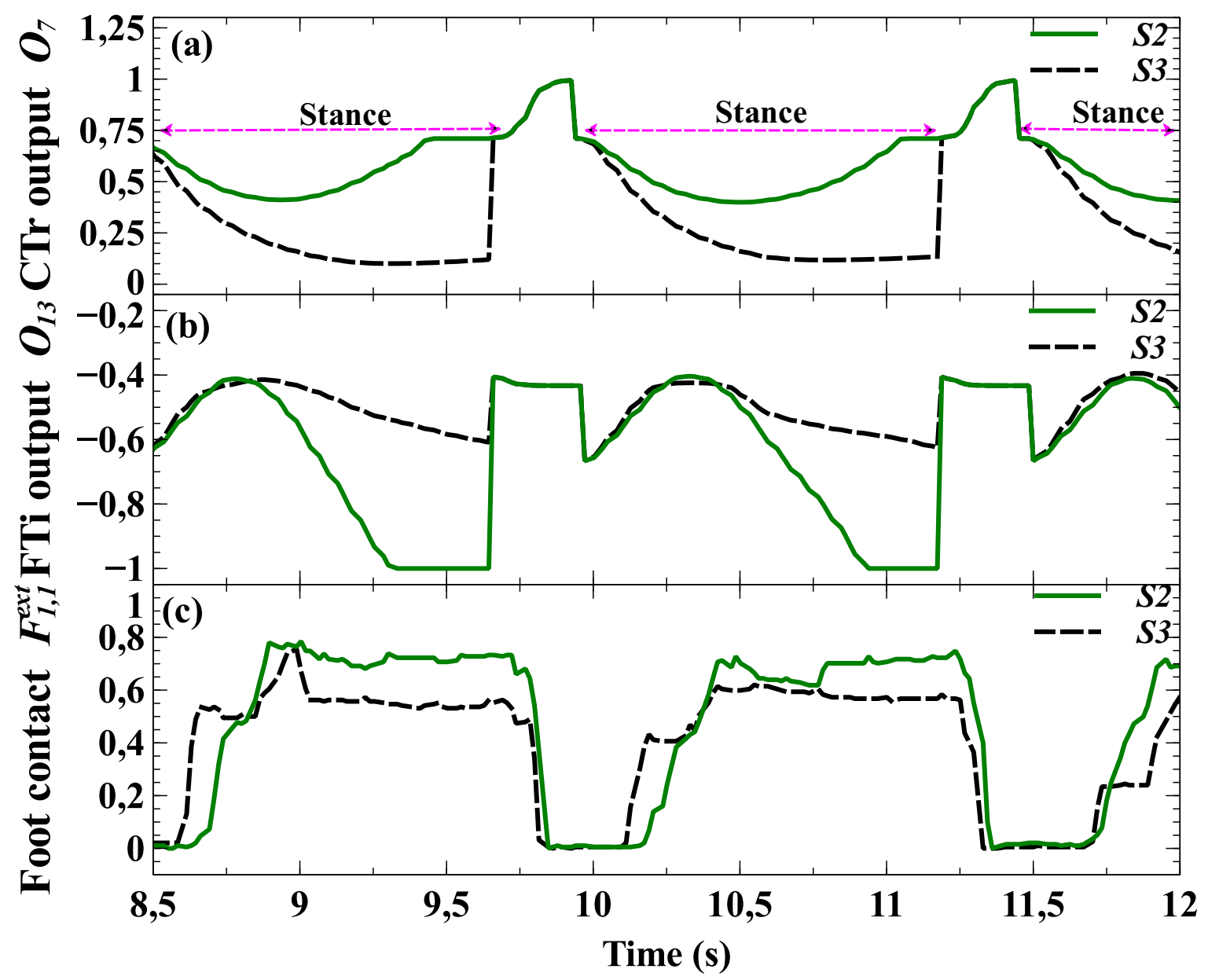

Figure 4.11: Joint outputs and foot force signals that resulted from two stiffness setups $S(2,3)$ during walking on sponge. (a) CTr joint outputs $O_{7}$. (b) FTi joint outputs $O_{13}$. (c) Analog signals of forces $F_{1,1}^{\text {ext }}$. The setup $S 2$ enables the hexapod robot to obtain more foot contact force during stance phases than the setup $S 3$.

plies with a finding of physiological experiments on insect locomotion (Spence et al., 2010; Spence, 2011). Owing to energy efficiency, insects (i.e., Blaberus discoidalis) always use the softer legs on soft elastic surfaces.

\subsection{Surface classification}

With the stiffness setup $S 2$, we ran the hexapod robot AMOS from coarse gravel (diameter $\varphi=16-25 \mathrm{~mm})$ to very coarse gravel $(\varphi=40-60 \mathrm{~mm})$. Its $\mathrm{CTr}$ and 


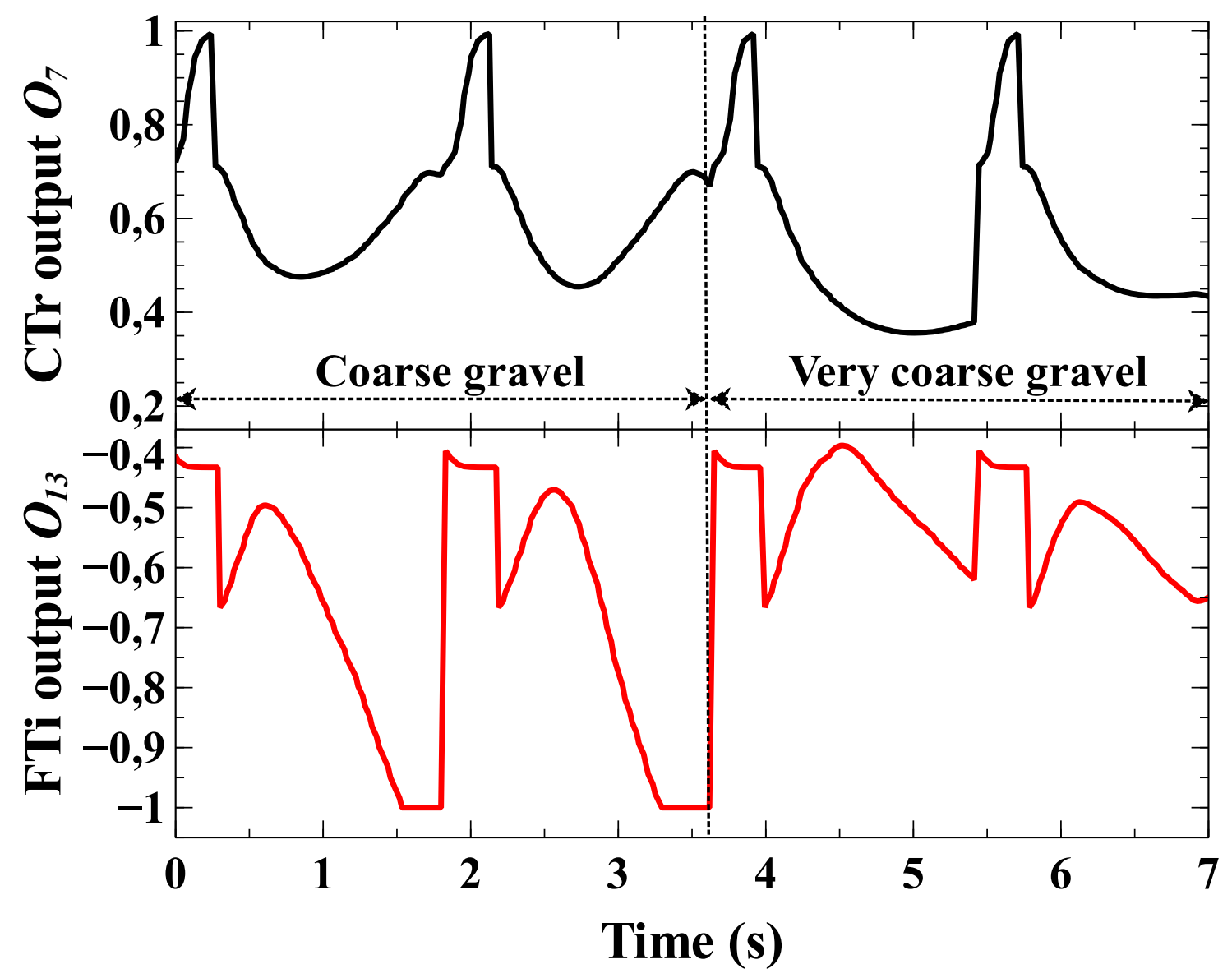

Figure 4.12: CTr and FTi joint signals (i.e., $O_{7}$ and $O_{13}$ ) of the right front leg. $O_{7}$ and $O_{13}$ show adaptive walking behaviors of the hexapod robot AMOS when it walked from coarse gravel (diameter $\varphi=16-25 \mathrm{~mm})$ to very coarse gravel $(\varphi=40-60 \mathrm{~mm})$.

FTi joint signals (i.e., $O_{7}$ and $O_{13}$ ) driving the right front leg show that AMOS can autonomously adapt its $\mathrm{CTr}$ and FTi joint motions during walking on different surfaces (see Fig. 4.12). In contrast to the CTr joint signal (e.g., see $O_{7}$ in Fig. 4.12), the FTi joint signal greatly varies from the coarse gravel to the very coarse gravel (e.g., see $O_{13}$ in Fig. 4.12). Other different FTi joint signals can be seen in Figs. 4.13 when AMOS walked on sponge and snow. Therefore, the FTi joint signals can be used to well classify the surfaces (see the classified surfaces in Fig. 4.14).

Five FTi joint signals (i.e., $O_{13}$ ) of the right front leg were obtained as order datum streams $F T_{j}(t)$ and used as the inputs of the cumulative moving averages (CMAs), when the hexapod robot AMOS was ran on five surfaces (see them in Fig. 4.14). The 


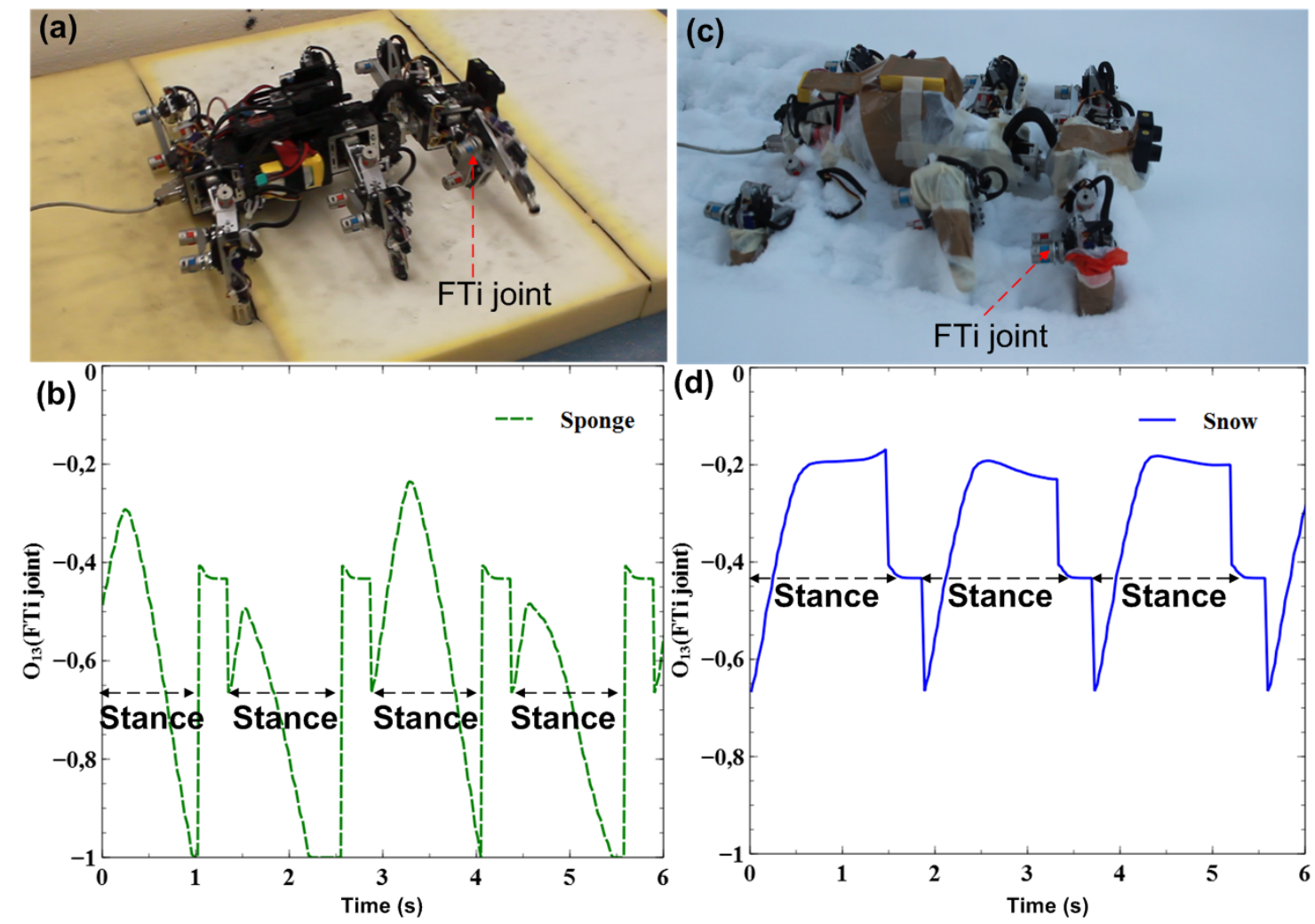

Figure 4.13: The hexapod robot AMOS walked on sponge and snow surfaces. (a) Snapshot of walking on sponge. (b) The FTi joint signal $O_{13}$ of walking on sponge. (c) Snapshot of walking on snow (d) The FTi joint signal $O_{13}$ of walking on snow.

outputs $C A_{j}(t)$ of the CMAs are given by:

$$
C A_{j}(t)=C A_{j}(t-\Delta t)+\frac{F T_{j}(t)-C A_{j}(t-\Delta t)}{\frac{t}{\Delta t}+1}, \Delta t \leq t \leq 14 s,
$$

where $j$ denotes the type of the surface, i.e., $j=1,2, \ldots, 4,5$ denote coarse gravel, very coarse gravel, floor, snow, and sponge surfaces. $t$ is a discrete time domain (i.e., $0 \mathrm{~s}-14 \mathrm{~s})$ at the interval $\Delta t \approx 0.019 \mathrm{~s}$. The initial value $C A_{j}(0)$ is set to $F T_{j}(0)$. The outputs $C A_{j}(t)$ shown in Fig.(4.15) are used as the sample signals for classifying the five surfaces.

Then we ran the hexapod robot AMOS on nine sets of the five surfaces. The total number of the runs is 45 . For each run, the output $C A(t)$ of each CMA is calculated by Eq.(4.13). The deviations $D E V_{j}$ between $C A(t)$ and $C A_{j}(t)$ are given by:

$$
\begin{array}{r}
D E V_{j}=\sum_{t=0}^{14}\left(\left|C A(t)-C A_{j}(t)\right|\right), j=1,2, \ldots, 5,6, \\
S C=\min \left(D E V_{j}\right), \text { when } \mathrm{h}=\mathrm{j},
\end{array}
$$



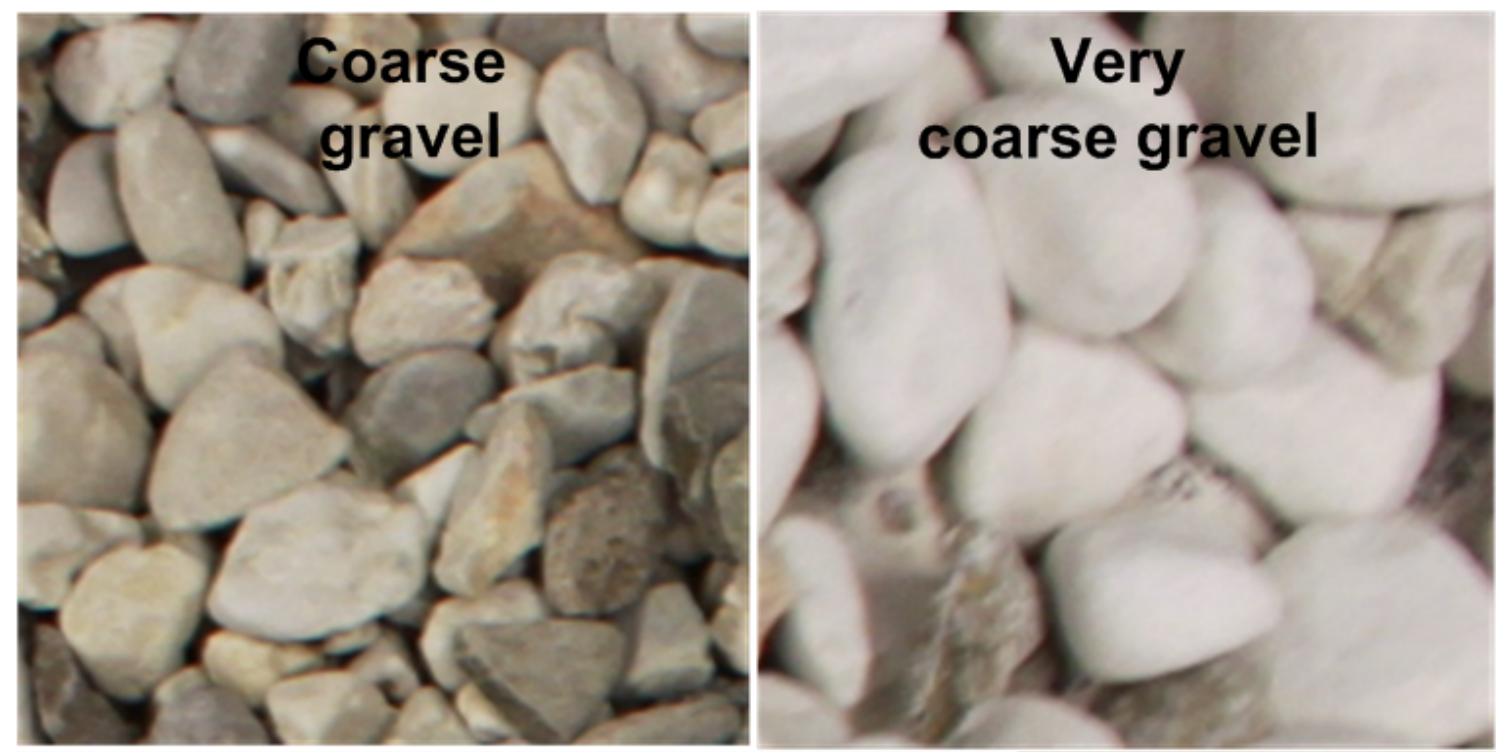

Floor

\section{Snow}

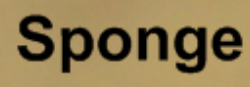

Figure 4.14: Five different surfaces used in the experimental classification. Since the floor, snow, and sponge surfaces do not have distinguishable features like gravel surfaces, it is very difficult for the legged robots to use the visual systems (e.g., cameras) (Filitchkin and Byl, 2012; Zenker et al., 2013) to classify them.

where $S C$ denotes the minimal value of the deviations $D E V_{j}$, and $h$ is the result of the classification for the run. $h=1,2, \ldots, 4,5$ denote coarse gravel, very coarse gravel, floor, snow, and sponge surfaces, respectively. For example, if $S C$ is $D E V_{1}$ (i.e., $h=1$ ), the result of the surface classification is coarse gravel.

The experimental statistics (i.e., the numbers of correct and wrong classification) of the surface classification is shown in Table 4.3. The success rate (SR) is used to evaluate the performance of surface classification, which is the ratio of the number of correctly classifying a given surface and the total number of classification on the surface. One can see that SR $=100 \%$ is found for the classification of all five experimental surfaces (see Table 4.3). We emphasize that the presented ability of well classifying the surfaces is due to the exploitation of adaptive joint signals (e.g., 


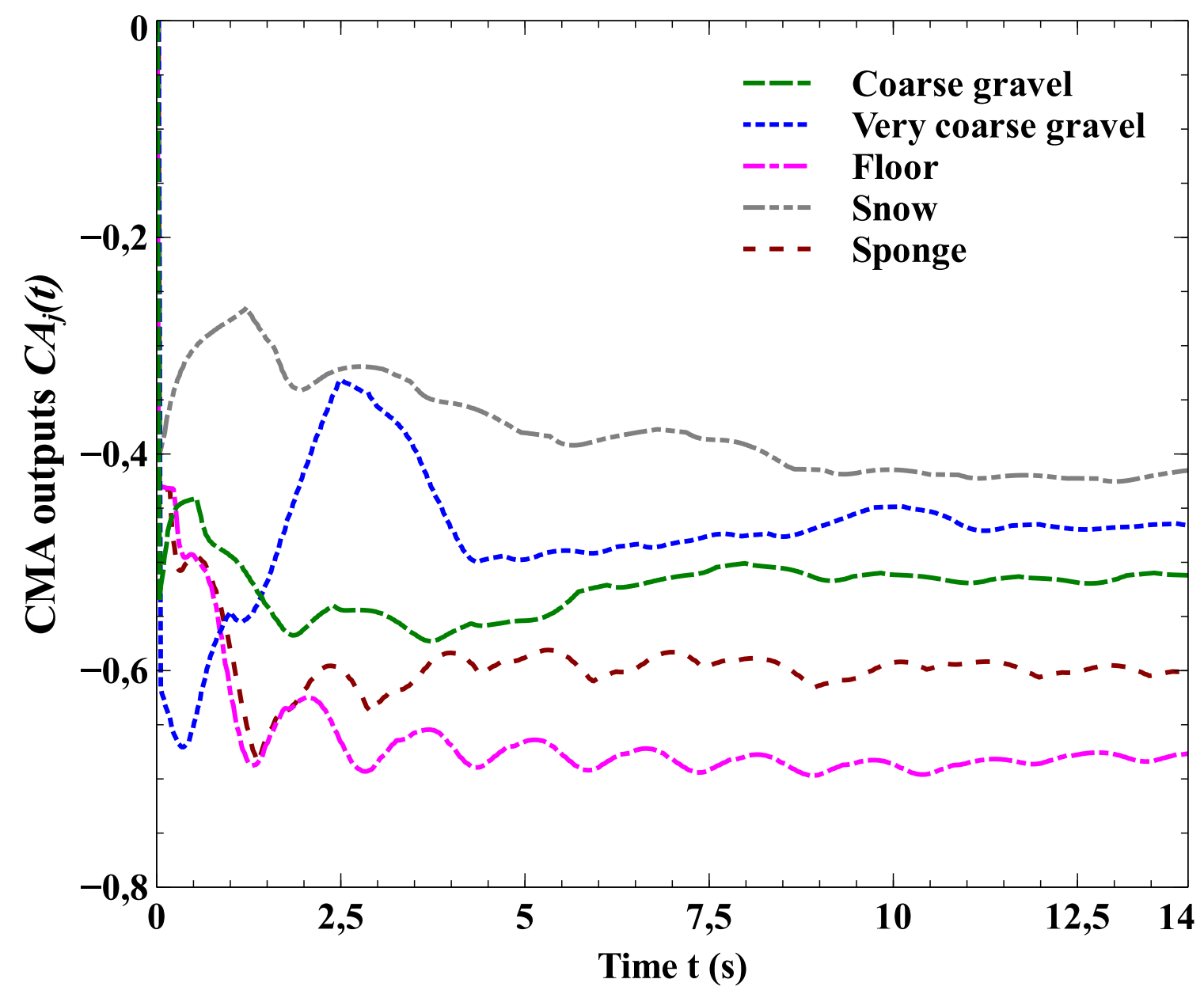

Figure 4.15: Cumulative moving average (CMA) outputs $C A_{j}(t)$.

see them in Fig. 4.12 ) generated by the virtual agonist-antagonist mechanisms (i.e., VAAMs, see Figs. 3.4). From this point of view, the VAAMs are one of the key components enabling the hexapod robot AMOS to well classify some types of surfaces where it walks on. Note that the key components include the modular neural network (MNN), the VAAMs, force sensing only at the end effectors of the legs, and the cumulative moving average algorithm.

\subsection{The benefits of the neuromechanical controller}

In this chapter, we present the neuromechanical controller (see Fig. 4.1) that enables the hexapod robot AMOS to achieve coordinated and variable compliant joint motions on rough surfaces (e.g., gravel). In the controller, coordinated joint motions 
Table 4.3: Experimental statistics of surface classification

\begin{tabular}{|l|c|c|c|c|c||c|}
\hline Classified as Samples & $\begin{array}{c}\text { Coarse } \\
\text { gravel }\end{array}$ & $\begin{array}{c}\text { Very } \\
\text { coarse } \\
\text { gravel }\end{array}$ & Floor & Snow & Sponge & SR \\
\hline Coarse gravel & $\mathbf{9}$ & 0 & 0 & 0 & 0 & $100 \%$ \\
\hline Very coarse gravel & 0 & $\mathbf{9}$ & 0 & 0 & 0 & $100 \%$ \\
\hline Floor & 0 & 0 & $\mathbf{9}$ & 0 & 0 & $100 \%$ \\
\hline Snow & 0 & 0 & 0 & $\mathbf{9}$ & 0 & $100 \%$ \\
\hline Sponge & 0 & 0 & 0 & 0 & $\mathbf{9}$ & $100 \%$ \\
\hline
\end{tabular}

are produced by the modular neural network (MNN, see Figs. 4.2) while the virtual agonist-antagonist mechanisms (VAAMs) generate the variable compliant joint motions by changing the stiffness parameters $K_{7-18}$ of the VAAMs (see Fig. 4.1). The generated variable compliant joint motions rely only on the force sensing at the end effectors of the legs. This makes the neuromechanical controller differ from classical compliance control mechanisms (e.g., variable stiffness actuators (VSAs) (Ham et al., 2009)) or methods (e.g., variable compliance control (Yang et al., 2011)) where bulky mechanisms (e.g., artificial muscles (Schmitt et al., 2012)) or complex force/torque sensing systems are required. In addition, the neuromechanical controller facilitates the integration of the proximo-distal gradient (see Fig. 2.5) to enhance stability (see Fig. 4.5) of legged robots under variable compliance control.

As a result, the compliant and adaptive joint motions (see Fig. 4.12) produced by the neuromechanical controller can help the six-legged robot AMOS to well classify the surfaces (see Table 4.3). Generally, surface classification has been implemented on legged robots relying on multiple sensing (e.g., current and angular positions) (Birnschein et al., 2009; Bartsch et al., 2012), force/torque sensing (Schmidt and Walas, 2013; Hoepflinger et al., 2010), vibration sensing (Bermudez et al., 2012; Giguere et al., 2006), and visual perception (Filitchkin and Byl, 2012; Zenker et al., 2013). Visual perception using the conventional techniques (Bay et al., 2008) cannot work well in classifying indistinguishable surfaces (e.g, snow, see Fig. 4.14). Owing to natural vibrations of legged robots, vibration sensing may not perform well for classifying surfaces either (Jacob Shill, 2012). Compared to multiple sensing, force sensing can still provide precise surface classification by using a simple algorithm. Force sensing embedded in the VAAMs of the neuromechanical controller allows AMOS to well classify five different surfaces. In addition to well classifying the surfaces, the compliant motions generated by the neuromechanical controller facilitate energy-efficient legged walking on different surfaces (see the COTs in Table 4.2). Moreover, the neuromechanical controller enables the hexapod robot (i.e., AMOS) to achieve more energyefficient walking (i.e., lower COTs) in contrast to neural controllers. For instance, the COT of a hexapod robot (i.e., Gregor I) is 70 when a cellular neural network is used to control its walking over an uneven surface (Arena et al., 2006). Combining the 
Table 4.4: The COTs under the controllers on two surfaces

\begin{tabular}{|l|c|c|}
\hline Controllers & Coarfaces gravel & Floor \\
\hline Neuromechanical controller & $\mathbf{1 8 . 1} \pm \mathbf{0 . 7}$ & $\mathbf{1 4 . 0} \pm \mathbf{0 . 4}$ \\
\hline Adaptive neural controller & $24.5 \pm 1.1$ & $21.9 \pm 1.2$ \\
\hline
\end{tabular}

neural networks with forward models can improve the energy efficiency of the hexapod robot (e.g., AMOS). For example, the combination of the modular neural network (i.e., MNN, see Figs. 4.2) and adaptive forward models enables AMOS to reduce the COT to 24.5 (see Table 4.4) when it walks over an uneven surface (i.e., coarse gravel) (Manoonpong et al., 2013b). Adaptive leg motions generated by the neural controllers of Gregor I and AMOS depend only on the changes of neural activities of the controllers for walking over uneven surfaces. By contrast, the neuromechanical controller relies on adjusting biomehcanical properties (e.g., the stiffness parameters $K_{(7-18)}$ ) of the muscle-like mechanisms (i.e., VAAMs), which enables AMOS to achieve more energy-efficient walking (i.e., lower COTs, see Table 4.4) (Xiong et al., 2014a). This is because the VAAMs of the neuromechanical controller facilitate proper leg penetrations into different surfaces that lead to gaining more foot contact force for propelling AMOS forward (see Figs. 4.10 and 4.11). The proper leg penetrations result from properly choosing the stiffness and damper parameters of the VAAMs that leads to insect-like muscle functions (i.e., brakes (Dickinson et al., 2000; Ahn and Full, 2002), see Figs. 3.11 (c) and (d)). Whereas other neural controllers like the adaptive neural controller (Manoonpong et al., 2013b) cannot achieve such leg penetrations due to the lack of the muscle-like mechanisms (e.g., VAAMs). 


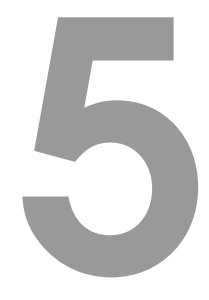

\section{Adaptive Neuromechanical Control for Online Compliance Adaptation}

In Chapter 4, we show that the neuromechanical controller enables the insect-like robot AMOS to achieve variable compliant joint motions that lead to energy-efficient walking on different surfaces. Such energy-efficient walking relies only on manually choosing the proper setups of the stiffness parameters of the virtual agonist-antagonist mechanisms (VAAMs) with respect to only one gait. However, generally legged animals select different optimal gaits for their speeds to minimize the energetic costs (Kar et al., 2003; Wampler and Popović, 2009). For example, legged insects select the wave and tripod gaits for their lower and high speeds to achieve energy-efficient walking (Nishi, 1998; Nishii, 2000), respectively. Interestingly, changing the modulatory input $S$ (see Eq.(4.2)) of the modular neural network (MNN) enables the insect-like robot AMOS to achieve different insect-like gaits for different speeds (see Figs. 5.1). At lower speeds, AMOS's legs follow the wave gaits where it moves only one leg at a time (see Figs. 5.1 (a) and (b)). In such slower gaits, AMOS starts from the hind legs (e.g., R3) to the middle legs (e.g., R2) and then to the front legs (e.g., R1) on either side. Such slower gaits are more stable, since there are more legs in contact with the ground that supports and stabilizes AMOS's walking. Whereas at higher speeds, the walking patterns (i.e., gaits) of AMOS are shifted as the duration of the stance phase is shortened. During the tripod gaits, for example, the front and hind legs on the right side move as a unit with the middle leg on the left side (see R1, R3, and L2 in Figs. 5.1 (h) and (i)). These three legs push the body forward while the rest of other three legs (see L1, L3, and R2 in Figs. 5.1 (h) and (i)) swing forward. The tripod gaits alternate between swing and stance with using two units (see the red and blue tripods in Figs. A.1) of the legs. They are faster and still stable, because the COM (center of mass) of the body can be maintained within the tripods of the leg supports (Ritzmann and Zill, 2013; Bender et al., 2011; Knight, 2011) (see Fig. A.1 (a)).

In addition to gait selections, actively adjusting muscle functions (e.g., brakes (Ahn and Full, 2002; Dickinson et al., 2000)) is another important characteristic of energy-efficient and adaptive legged locomotion (Tytell et al., 2011; Spence et al., 2010; Farley et al., 1998; Ferris et al., 1998). For example, insects rely mainly on the 

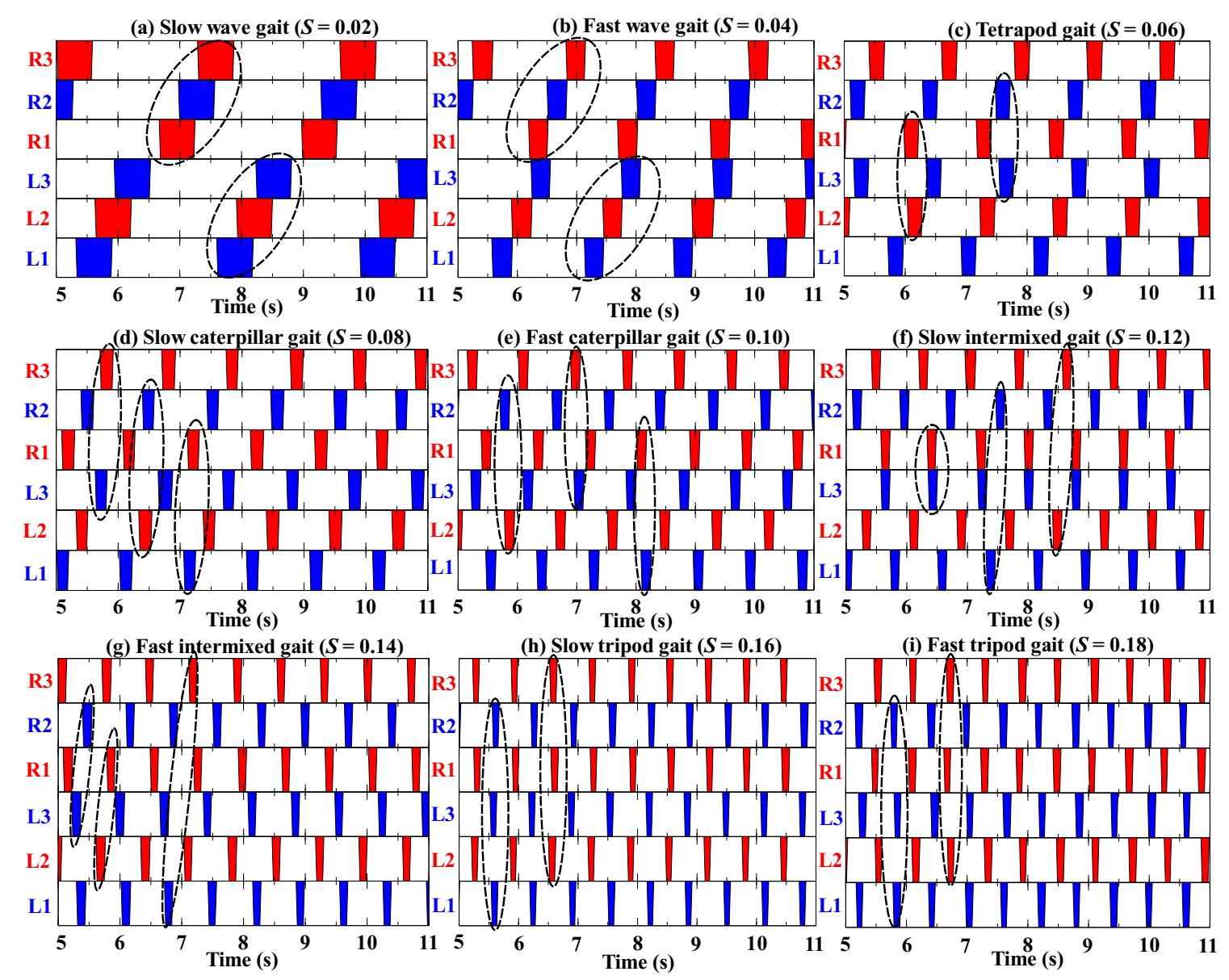

Figure 5.1: Nine gaits generated by the modular neural network (MNN). They are observed from the outputs (e.g., see Fig. 4.3) of the TC motor neurons of the MNN (see Fig. $4.2(\mathrm{~d})$ ). Red or blue areas indicate no ground contacts during swing phases while white areas refer to ground contacts during stance phases (e.g., see Fig. 4.3). As frequency increases, some legs step in pair (see dashed enclosures). Nine insect-like gaits are achieved by changing the modulatory input $S$ (see Eq.(4.2)) of the MNN. The speeds of AMOS's leg motions increase with the increasing modulatory input $S$. Abbreviations are: $\mathrm{R}(1,2,3)=$ Right (Front, Middle, Hind) leg, L(1, 2, 3) = Left (Front, Middle, Hind) leg.

tunable muscle functions to overcome and mover over rough terrain (Sponberg and Full, 2008; Kukillaya and Holmes, 2009; Sponberg et al., 2011). Therefore the adaptive neuromechanical control on the insect-like robot AMOS involves the modular neural network (MNN, see Figs. 4.2) for generating insect-like gaits (see Figs. 5.1), several 


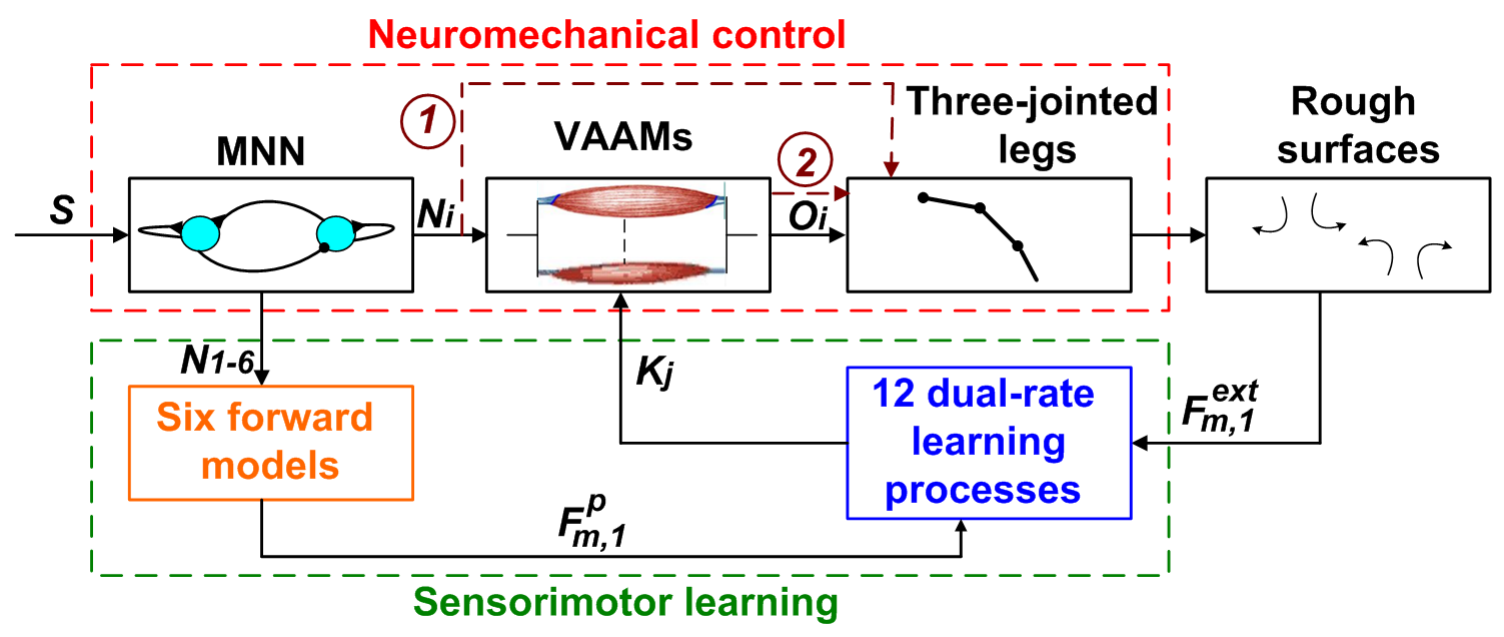

Figure 5.2: Neuromechanical control coupled with sensorimotor learning applied to the hexapod robot AMOS. Via neural outputs $N_{i}(i=1,2, \ldots, 17,18)$, the modular neural network (MNN) activates the virtual agonist-antagonist mechanisms (VAAMs) that generate position commands (i.e., $O_{i}$ ) to move the three-jointed legs of AMOS. The legs then interact with rough surfaces (e.g., gravel), which produce force feedback (i.e., $\left.F_{m, 1}^{e x t}\right)(m=1,2, \ldots, 5,6)$. Besides, the six forward models predict expected force feedback (i.e., $F_{m, 1}^{p}$ ) of the legs based on the outputs of the MNN. Using $F_{m, 1}^{e x t}$ and $F_{m, 1}^{p}$ as the inputs, 12 dual-rate learning processes actively tune 12 stiffness parameters $K_{j}$ (i.e., $j=7,8, \ldots, 17,18$ ) of the VAAMs driving the CTr and FTi joints. In addition to descending commands (i.e., $S, N_{i}$, and $O_{i}$ ), there are the other two ways (see (1) and (2)) to generate the joint outputs $O_{i}$. These three ways refer to the proximo-distal gradient (see more details in Fig. 4.1).

virtual agonist-antagonist mechanisms (VAAMs, see Figs. 3.4) for tunable insectlike muscle functions (e.g., the brakes, see Figs. 3.11 (c) and (d)), and sensorimotor learning for self-adjusting the stiffness parameters $K_{(7-18)}$ of the VAAMs (see Fig. 5.2). Such adaptive control can be modeled as a set of distributed and closed loops with feed-forward and feedback pathways (Roth et al., 2014; Revzen et al., 2009; Ting et al., 2009). For the feed-forward pathways, the controller not only consists of feedforward control via descending commands (i.e., $S, N_{i}$, and $O_{i}$ ) from the MNN to the VAAMs and the six legs, but also includes six forward models (Webb, 2004) for predicting force sensing (i.e., $F_{m, 1}^{p}$ ) of the six legs. The feed-forward ways start at the modulatory input $S$ (see Eq.(4.2)) of the MNN that is set to nine values for AMOS to achieve nine insect-like gaits (see Figs. 5.1), respectively. In the feedback pathway, there is force sensing (i.e., $\left.F_{m, 1}^{e x t}\right)$ at the end effectors of the legs. Using $F_{m, 1}^{p}$ and $F_{m, 1}^{e x t}$ as the inputs, 12 dual-rate learning processes can actively tune the stiffness parameters $K_{j}$ (i.e., $K 1_{6 \times 1}$ and $K 2_{6 \times 1}$ in Eqs.(4.5) and (4.8)) of the muscle-like components (i.e., VAAMs) driving the 12 joints of the legs. This leads to adaptive compliant joint 
motions that accommodate different surfaces and gaits.

\subsection{Sensorimotor learning for adaptive compliant joint motions}

The adaptive compliant joint motions of AMOS are achieved by actively adjusting the stiffness parameters $K 1_{6 \times 1}$ and $K 2_{6 \times 1}$ (see Eqs.(4.5) and (4.8)) of the passive elements (i.e., PEs) of the VAAMs driving the FTi and CTr joints. Here, we apply sensorimotor learning for online adjusting $K 1_{6 \times 1}$ and $K 2_{6 \times 1}$ at every time step $\Delta t$ (i.e., $\Delta t=0.019(s)$ ). For each leg, there are two dual-rate learning processes and a forward model (see Figs. 5.3 (a) and (b)) for the CTr and FTi joints. The forward model uses the outputs (i.e., $\left.O_{m}(t)\right)$ driving the TC joints to predict foot force signals (i.e., $\left.F_{m, 1}^{p}(t), m=1,2, \ldots, 5,6\right)$. Specifically, $F_{m, 1}^{p}(t)$ will gradually increase to 1 when $O_{m}(t)$ is decreasing (e.g., see $F_{4,1}^{p}(t)$ and $O_{4}(t)$ in Fig. $\left.5.4(\mathrm{a})\right) . F_{m, 1}^{p}(t)$ are given by:

$$
\begin{gathered}
F_{m, 1}^{p}(t+\Delta t)=0.2 G_{m, 1}(t)+0.8 F_{m, 1}^{p}(t), \\
G_{m, 1}(t)= \begin{cases}1, & O_{m}(t+\Delta t)<O_{m}(t) . \\
0, & O_{m}(t+\Delta t)>O_{m}(t) .\end{cases}
\end{gathered}
$$

The matrix $e_{6 \times 1}(t)$ of errors between expected and real foot force signals is:

$$
\begin{array}{r}
e_{6 \times 1}(t)=F_{6 \times 1}^{e x t}(t)-F_{6 \times 1}^{p}(t), \\
e_{6 \times 1}(t)=\left[e_{1}(t), e_{2}(t), \ldots, e_{5}(t), e_{6}(t)\right]^{T},
\end{array}
$$

where $F_{6 \times 1}^{e x t}(t)$ is the matrix of the real foot force signals, i.e., $F_{6 \times 1}^{e x t}(t)=f_{1-6}^{e x t}(t)$ (see Fig.4.4 (a)). $F_{6 \times 1}^{p}(t)$ is the matrix of the predicted foot force signals, i.e., $F_{6 \times 1}^{p}(t)=$ $f_{1-6}^{p}(t)$.

An error (e.g., $\left.e_{4,1}(t)\right)$ is used as the input to a dual-rate learning process. For reducing the error (e.g., see $e_{4,1}(t)$ at Fig.5.4 (b)), the process adjusts the stiffness parameter (e.g., $K 1_{4,1}(t)$ ) of the PEs driving the FTi joint in each leg (see Fig. 5.3(a)). Each learning process consists of a fast learner and of a slow learner. Both learners are modeled as linear systems acting in parallel. The fast one learns compensating the error more quickly, is indicated by a higher learning rate, i.e., $B 1_{f}>B 1_{s}$. Whereas, the slow one retains previous states much better, is indicated by a high retention factor, i.e., $A 1_{f}<A 1_{s}$. Therefore, the matrix $K 1_{6 \times 1}(t)$ of stiffness parameters for the FTi joints is given by:

$$
\begin{array}{r}
K 1_{6 \times 1}^{f}(t+\Delta t)=A 1_{f} K 1_{6 \times 1}^{f}(t)+B 1_{f} e_{6 \times 1}(t), \\
K 1_{6 \times 1}^{s}(t+\Delta t)=A 1_{s} K 1_{6 \times 1}^{s}(t)+B 1_{s} e_{6 \times 1}(t), \\
K 1_{6 \times 1}(t+\Delta t)=K 1_{6 \times 1}^{f}(t+\Delta t)+K 1_{6 \times 1}^{s}(t+\Delta t),
\end{array}
$$




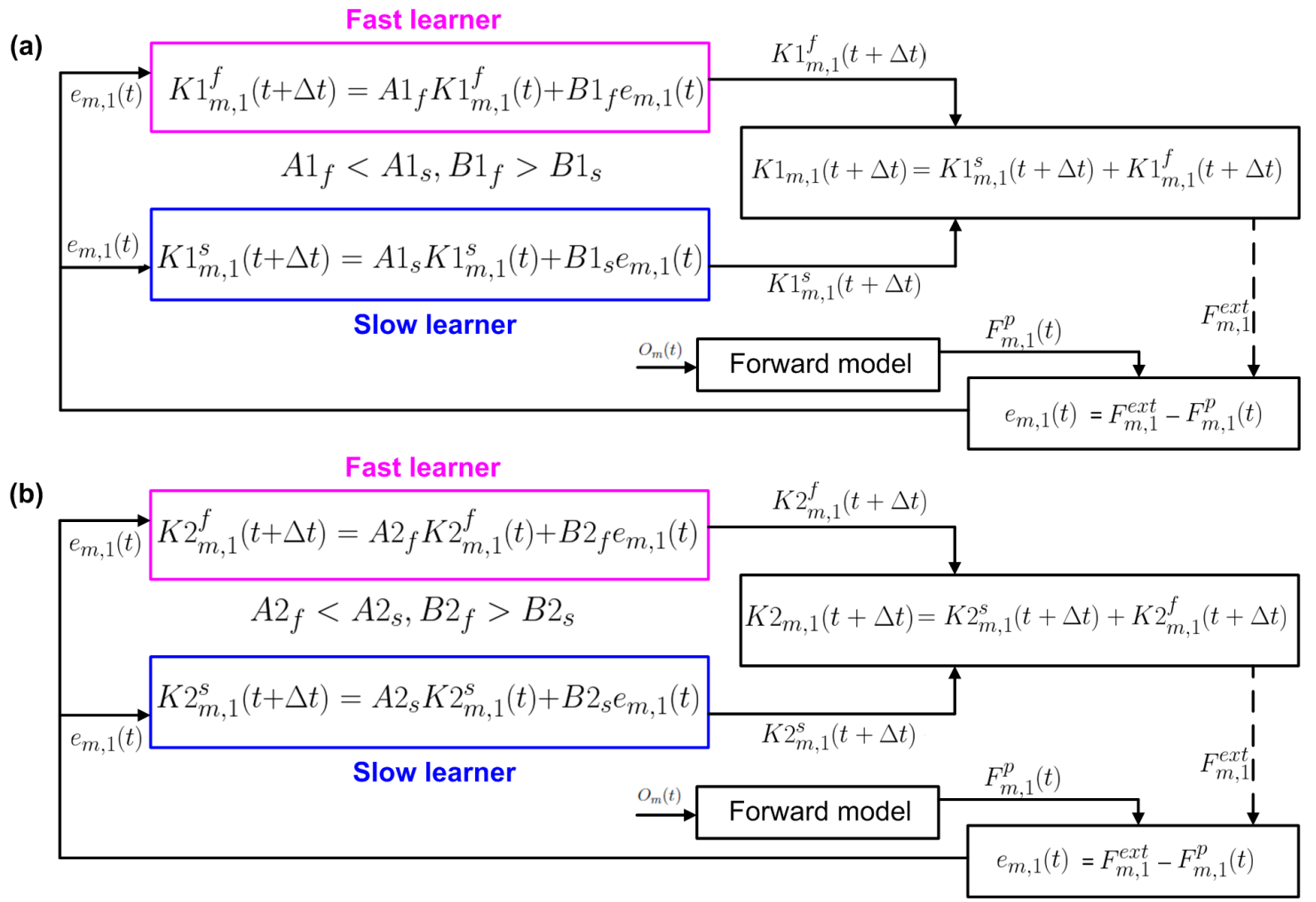

Figure 5.3: Sensorimotor learning for stiffness parameters $K 1_{m, 1}$ and $K 2_{m, 1} \cdot K 1_{m, 1}$ and $K 2_{m, 1}(m=1,2, \ldots, 5,6)$ are stiffness parameters of the passive elements (i.e., PEs) driving the FTi and CTr joints of the AMOS's legs. For each leg, there are two dual-rate learning processes for adjusting stiffness parameters (e.g., $K 1_{4,1}$ and $K 2_{4,1}$ ) by using expected and real foot force signals (e.g., $F_{4,1}^{p}$ and $F_{4,1}^{e x t}$ ). The expected foot force signal (e.g., $F_{4,1}^{p}$ ) is predicted by a forward model based on an output (e.g., $O_{4}$ ) driving the TC joint. Each dual-rate learning process consists of a fast learner and of a slower learner acting in parallel. (a) A dual-rate learning process for stiffness parameters $K 1_{m, 1}$. The parameters of the two learners are set as: $A 1_{f}=0.59$, $A 1_{s}=0.992, B 1_{f}=0.378$, and $B 1_{s}=0.036$. (b) A dual-rate learning for stiffness parameters $K 2_{m, 1}$. The parameters of the two learners are set as: $A 2_{f}=0.59$, $A 2_{s}=0.992, B 2_{f}=0.882$, and $B 2_{s}=0.084$.

where $K 1_{m, 1}^{f}(t+\Delta t)$ are the outputs of fast learners, and $K 1_{m, 1}^{s}(t+\Delta t)$ are the outputs of slow learners. Note that the value of $A 1_{f}$ and $A 1_{s}$ are from (Smith et al., 2006), and $B 1_{f}$ and $B 1_{s}$ are empirically chosen (see all values in Fig. 5.3(a)).

Similarly, the matrix $K 2_{6 \times 1}(t)$ of stiffness parameters (see Fig. 5.3(b)) for the CTr 
joints is given by:

$$
\begin{array}{r}
K 2_{6 \times 1}^{f}(t+\Delta t)=A 2_{f} K 2_{6 \times 1}^{f}(t)+B 2_{f} e_{6 \times 1}(t), \\
K 2_{6 \times 1}^{s}(t+\Delta t)=A 2_{s} K 2_{6 \times 1}^{s}(t)+B 2_{s} e_{6 \times 1}(t), \\
K 2_{6 \times 1}(t+\Delta t)=K 2_{6 \times 1}^{f}(t+\Delta t)+K 2_{6 \times 1}^{s}(t+\Delta t),
\end{array}
$$

where $K 2_{m, 1}^{f}(t+\Delta t)$ are the outputs of fast learners, and $K 2_{m, 1}^{s}(t+\Delta t)$ are the outputs of slow learners. Note that the value of $A 2_{f}$ and $A 2_{s}$ are from (Smith et al., 2006), and $B 2_{f}$ and $B 2_{s}$ are empirically chosen (see all values in Fig. 5.3(b)). The Eqs.(5.3) and (5.4) are written in terms of time $t$ different from the equations in (Smith et al., 2006) and (Wolpert et al., 2011) formulated according to trial number $n$.

\subsection{Adaptive compliant joint motions for a gait and a surface}

For each leg, there are two learning processes coupled with a forward model (see Fig. 5.3) for adjusting the stiffness parameters (e.g., $K 1_{4,1}$ and $K 2_{4,1}$ ). At the left front leg, for example, there are two outputs (i.e., $K 2_{4,1}^{f}$ and $K 2_{4,1}^{s}$ ) of fast and slow learners acting in parallel, which contribute to stiffness parameter $K 2_{4,1}$ (see Fig.5.4 (c)). One can see that the fast one learns $K 2_{4,1}^{f}$ more rapidly, which leads to smaller oscillations (see green dashed line in Fig. 5.4 (c)). By contrast, the slow one retains $K 2_{4,1}^{s}$ better, thereby leading to the convergence (see red dashed line in Fig. 5.4 (c)). This is because the retention factor $A 2_{f}=0.59$ of the fast learner is lower than $A 2_{s}=0.992$ of the slow learner (see Eq.(5.4)). Moreover, the fast learner is more sensitive to the perturbations (i.e., stance phases) after learning (see Fig. 5.5), compared to the slow learner. This is because the learning rate $B 2_{f}=0.882$ of the fast learner is higher than $B 2_{s}=0.084$ of the slow learner (see Eq.(5.4)). The combination of the slow and fast learners enables the stiffness parameters (e.g., $K 2_{4,1}$ ) to achieve global convergences and local oscillatory stiffness responds (see Figs. 5.4 (c) and 5.5), which lead to stable and adaptive compliant hexapedal walking on rough surfaces. Furthermore, the stiffness parameters (e.g., $K 2_{4,1}$ ) during swing phases are higher than them during stance phases (see Fig. 5.5), since they (during the swing phases) are kept as the stiffness parameters from the previous stance phases. Note that sensorimotor learning (see Eqs. 5.1, 5.3, and 5.4) is not applied to adaptively control the joints during swing phases, since there is only feed-forward neural control (see Eq.(4.11)) on the joints during swing phases. Whereas during stance phases, the stiffness parameters (e.g., $K 2_{4,1}$ ) initially decrease and increase afterwards (e.g., see Fig. 5.5). This is because the muscle-like mechanisms (i.e., VAAMs) need to initially soften the joints to absorb impacts of external loads, and afterwards stiffen them to 


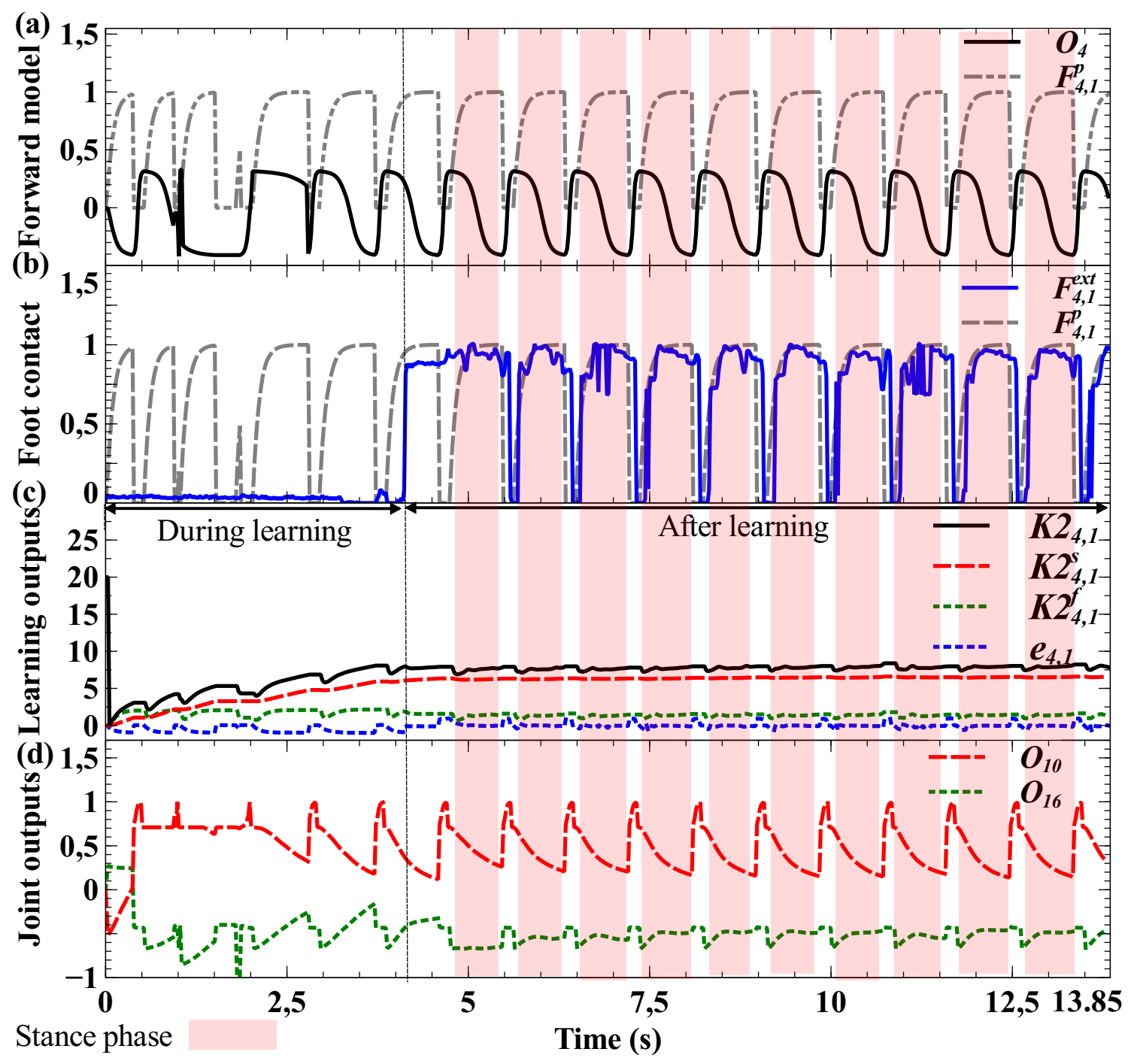

Figure 5.4: Sensorimotor learning for adjusting stiffness parameter $K 2_{4,1}$. Here the gait is fast caterpillar (i.e., the modulatory input $S=0.10$ ). (a) Forward model. The output $O_{4}(t)$ driving the TC joint is applied to predict the foot force signal $F_{4,1}^{p}(t)$ (see Eq.(5.1)). (b) Contact forces. $F_{4,1}^{e x t}(t)$ and $F_{4,1}^{p}$ are the real and predicted contact forces. (c) Learning the stiffness parameter $K 2_{4,1} . K 2_{4,1}$ is the sum of the outputs (i.e., $K 2_{4,1}^{f}$ and $K 2_{4,1}^{s}$ ) of a fast learner and a slow learner using the error $e_{4,1}$ between $F_{4,1}^{e x t}$ and $F_{4,1}^{p}$ (see Eq.(5.4)). The adjustment of stiffness parameter $K 1_{4,1}$ driving the FTi joint in the left front leg is shown in Fig.5.6 (a) (i.e., see LF). (d) $O_{10}$, and $O_{16}$ are the outputs controlling the positions of the CTr and FTi joints in the left front leg. 


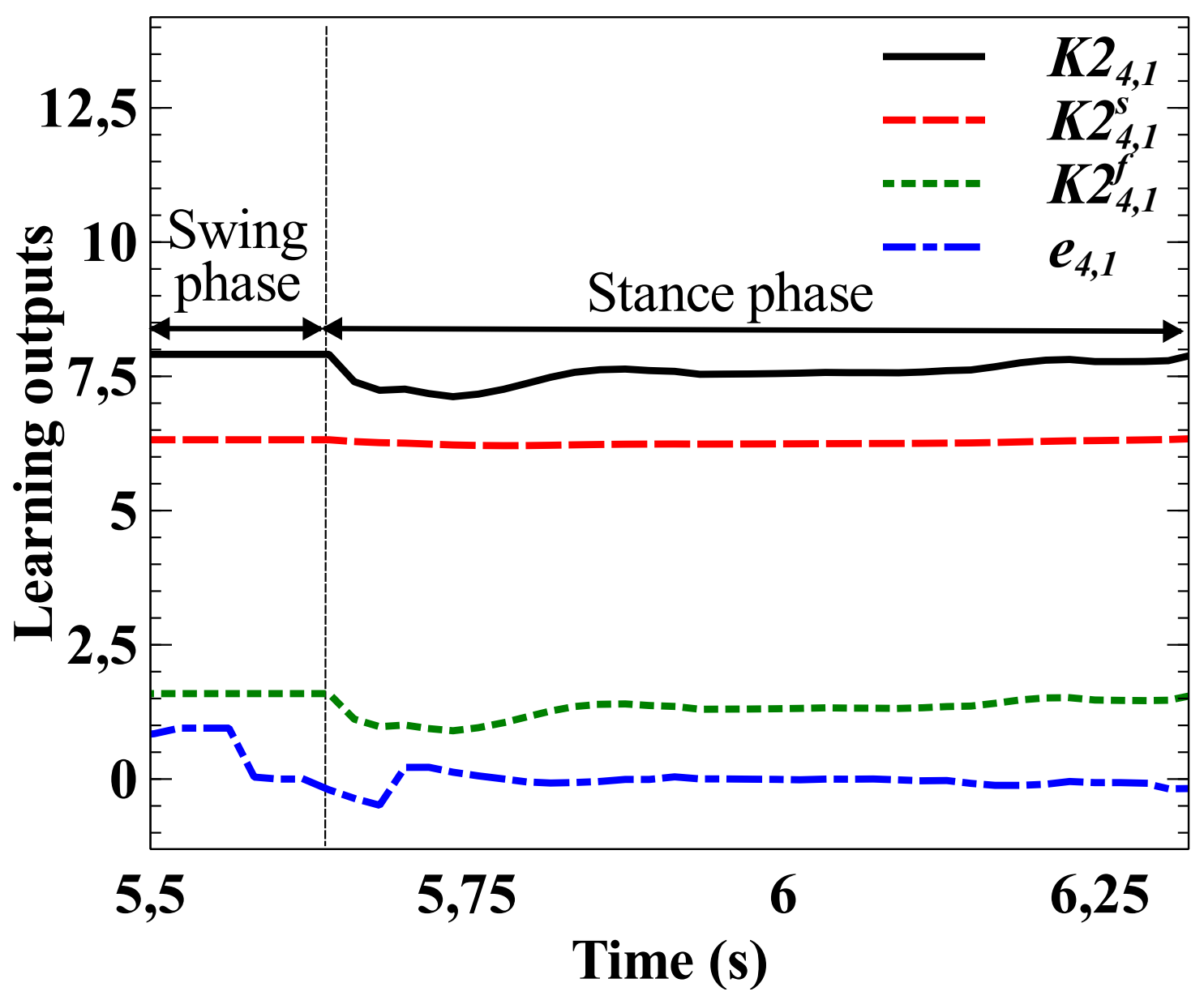

Figure 5.5: Sensorimotor learning for adjusting stiffness parameter $K 2_{4,1}$ during a swing and stance phases (see more details at Figs. 5.4). The figure is clipped from Fig. 5.4 (c).

obtain more forces to move forward. Similarly, the PEs of the VAAMs also soften and stiffen the FTi joints during stances phases (see Fig. 5.6 (a)). In other words, the VAAMs stiffen when the external load increases (i.e., stance phases). This property of the VAAMs is comparable to that of biological muscles, which become stiff when the external load increases (Lappin et al., 2006; Nishikawa et al., 2007). The video of the experiment can be seen at http://www. youtube.com/watch?v=B0v5D9yiRH4. Note that AMOS had difficulties to walk on all experimental surfaces when only fast or slow learners were used to tune stiffness parameters $K 1_{6 \times 1}$ and $K 2_{6 \times 1}$. The video of the experiment can be seen at http://www . youtube.com/watch?v=Lq22FibYLE4. This is because the slow or fast learners allow only for global convergences or local oscillatory 


\subsection{ADAPTIVE COMPLIANT JOINT MOTIONS FOR A GAIT AND A}

SURFACE

stiffness responds (see Fig. 5.5). Whereas combining the slow and fast learners, the dual-rate learners enable $K 1_{m, 1}$ and $K 2_{m, 1}$ to achieve global convergences (see Fig. 5.4 (c)) and local oscillatory stiffness responds (see Figs. 5.6), thereby leading to stable and adaptive walking on different surfaces. Moreover, the ranges of the stiffness parameters $K 1_{m, 1}$ and $K 2_{m, 1}$ vary between hind and non-hind legs. Lower $K 1_{(3,6), 1}$ and higher $K 2_{(3,6), 1}$ (see LH and RH in Figs. 5.6) press the hind legs more down, which enhance locomotion stability, compared to the front and middle legs. This is because the mass of AMOS mainly concentrates on its hind part. Furthermore, the values of $B(1,2)_{f}$ and $B(1,2)_{s}$ are empirically chosen to produce proper stiffness parameters $K 1_{m, 1}$ and $K 2_{m, 1}$ (see Figs. 5.3), which leads to appropriate (e.g., smooth) compliant joint motions of AMOS. For example, the compliant CTr joint motions are smoother (see $K 2_{m, 1}=9.0$ in Fig. 5.7) when the parameters $K 2_{m, 1}$ of their driving VAAMs are self-adjusted between 6.5 and 13.5 (see Fig. $5.6(\mathrm{~b})$ ).
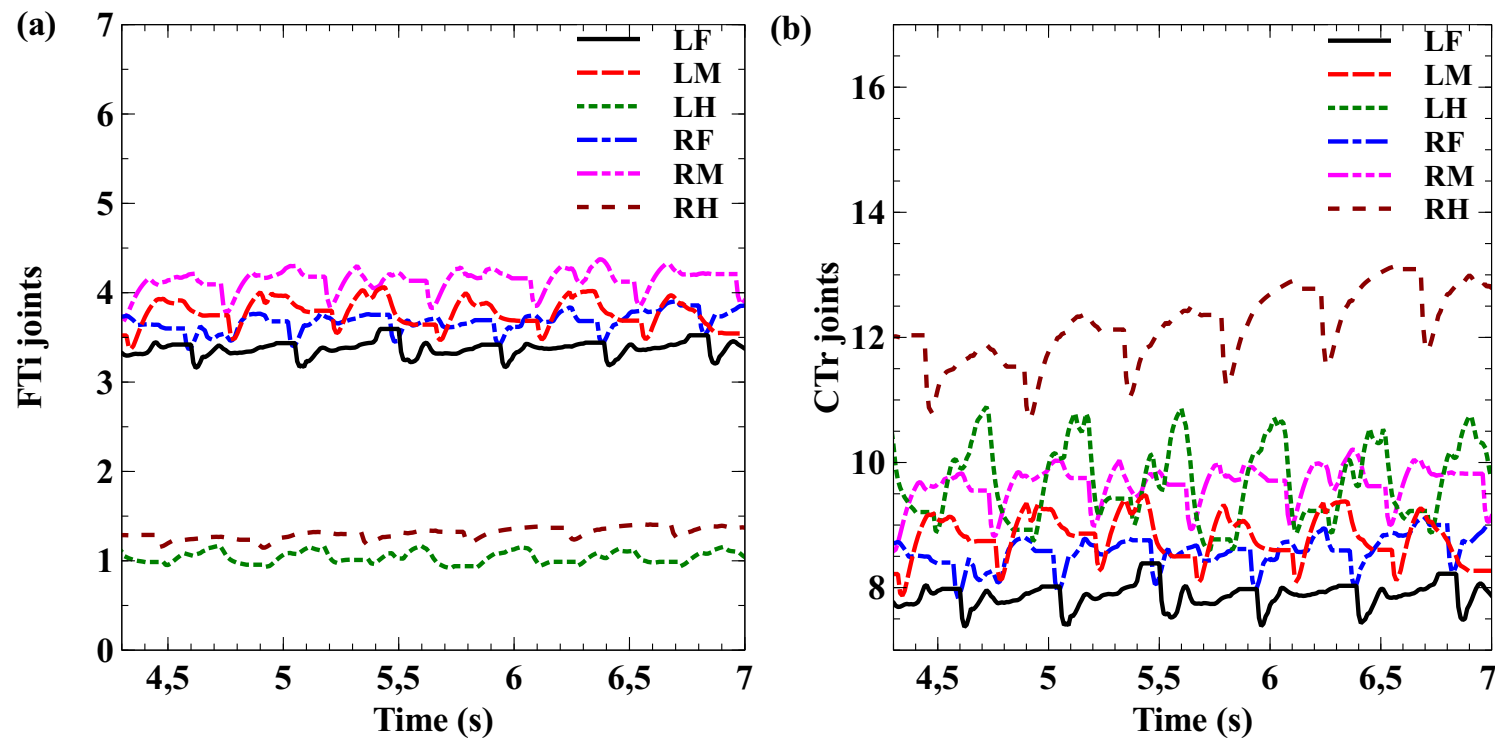

Figure 5.6: Stiffness parameters $K 1_{m, 1}$ and $K 2_{m, 1}$ after sensorimotor learning $(m=$ $1,2, \ldots, 5,6)$. Here the gait is fast caterpillar (i.e., the modulatory input $S=0.10$ ). (a) Stiffness parameters $K 1_{m, 1}$ of the VAAMs that drive the FTi joints. Abbreviations are: $\mathrm{R}(\mathrm{F}, \mathrm{M}, \mathrm{H})=K 1_{(1,2,3), 1}, \mathrm{~L}(\mathrm{~F}, \mathrm{M}, \mathrm{H})=K 1_{(4,5,6), 1}$. (b) Stiffness parameters $K 2_{m, 1}$ of the VAAMs that drive the CTr joints. Abbreviations are: $\mathrm{R}(\mathrm{F}, \mathrm{M}, \mathrm{H})=K 2_{(1,2,3), 1}$, $\mathrm{L}(\mathrm{F}, \mathrm{M}, \mathrm{H})=K 2_{(4,5,6), 1}$. 


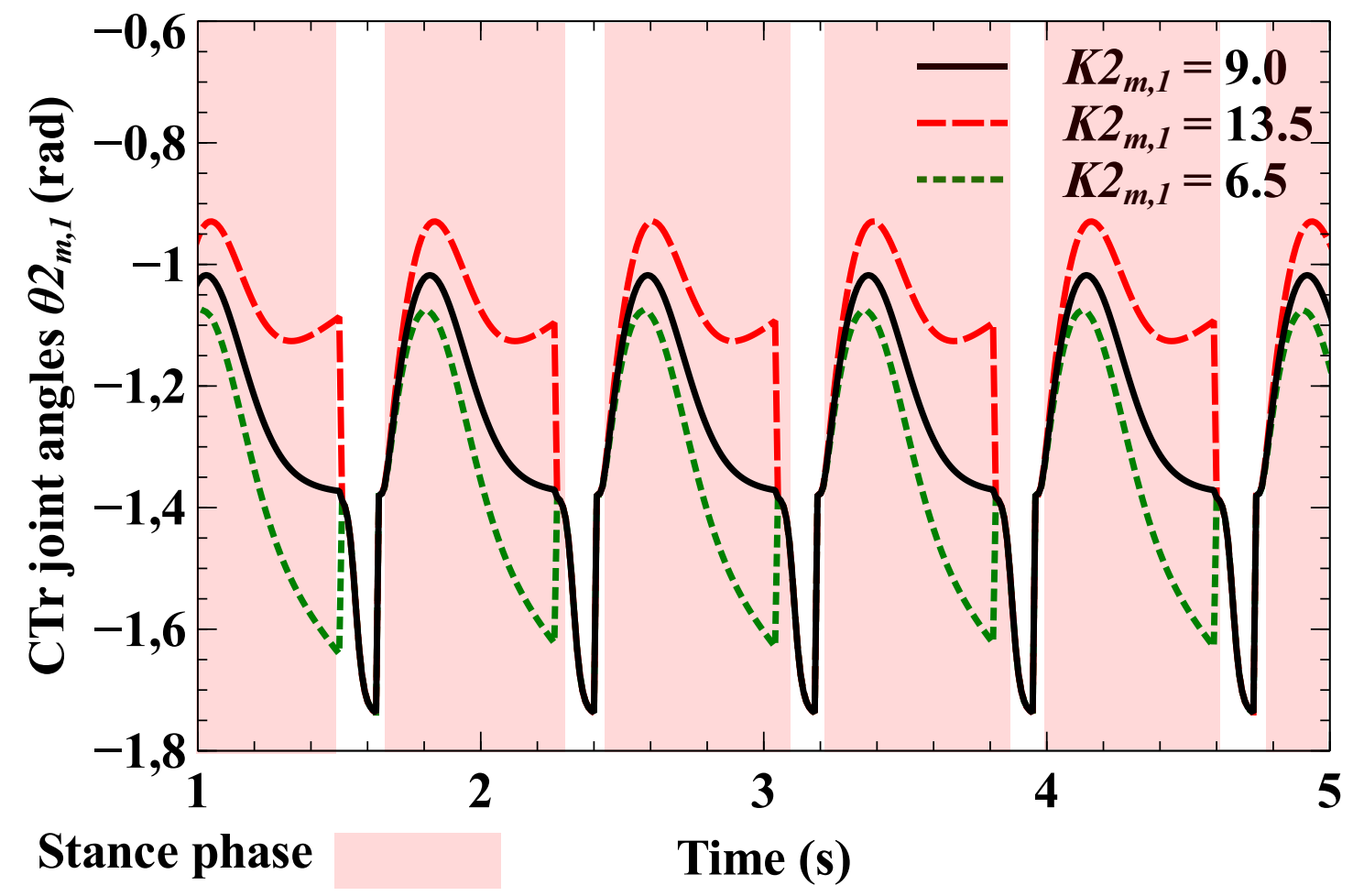

Figure 5.7: The smoothness of the compliant CTr joint motions that varies with the stiffness parameters $K 2_{m, 1}$.

\subsection{Adaptive compliant joint motions for different gaits}

Actively adjusting stiffness parameters $K 1_{6 \times 1}$ and $K 2_{6 \times 1}$ allows AMOS to achieve adaptive compliant joint motions that accommodate different gaits. AMOS, for instance, walked on fine gravel when the slow wave (i.e., $S=0.02$ ) and fast caterpillar (i.e., $S=0.10$ ) gaits were chosen (see Figs. 5.1 (a) and (e)), respectively. One can see that AMOS stiffens its CTr and FTi joints during stance phases, no matter which gait is chosen (e.g., see the red and gray areas in Figs. 5.8 (c) and (e)). The video of the experiments can be seen at http://www . youtube. com/watch?v=tmqr65qIOTY. Moreover, the slow wave gait enables CTr and FTi joints to achieve stiffer motions that result from larger $K 1_{m, 1}$ and $K 2_{m, 1}$ (e.g., see green dashed circles in Figs. 5.8 (c) and (e)), compared to the fast caterpillar gait. That is, AMOS stiffens the legs during stance phases when the speed of its leg motion is reduced from the fast gait to the slow gait. This result is comparable to the finding of physiological experiments, which had shown that at low speed animals walk by vaulting stiffer legs (Alexander, 2003; Holmes et al., 2006). By contrast, AMOS softens its legs when the speed of its 


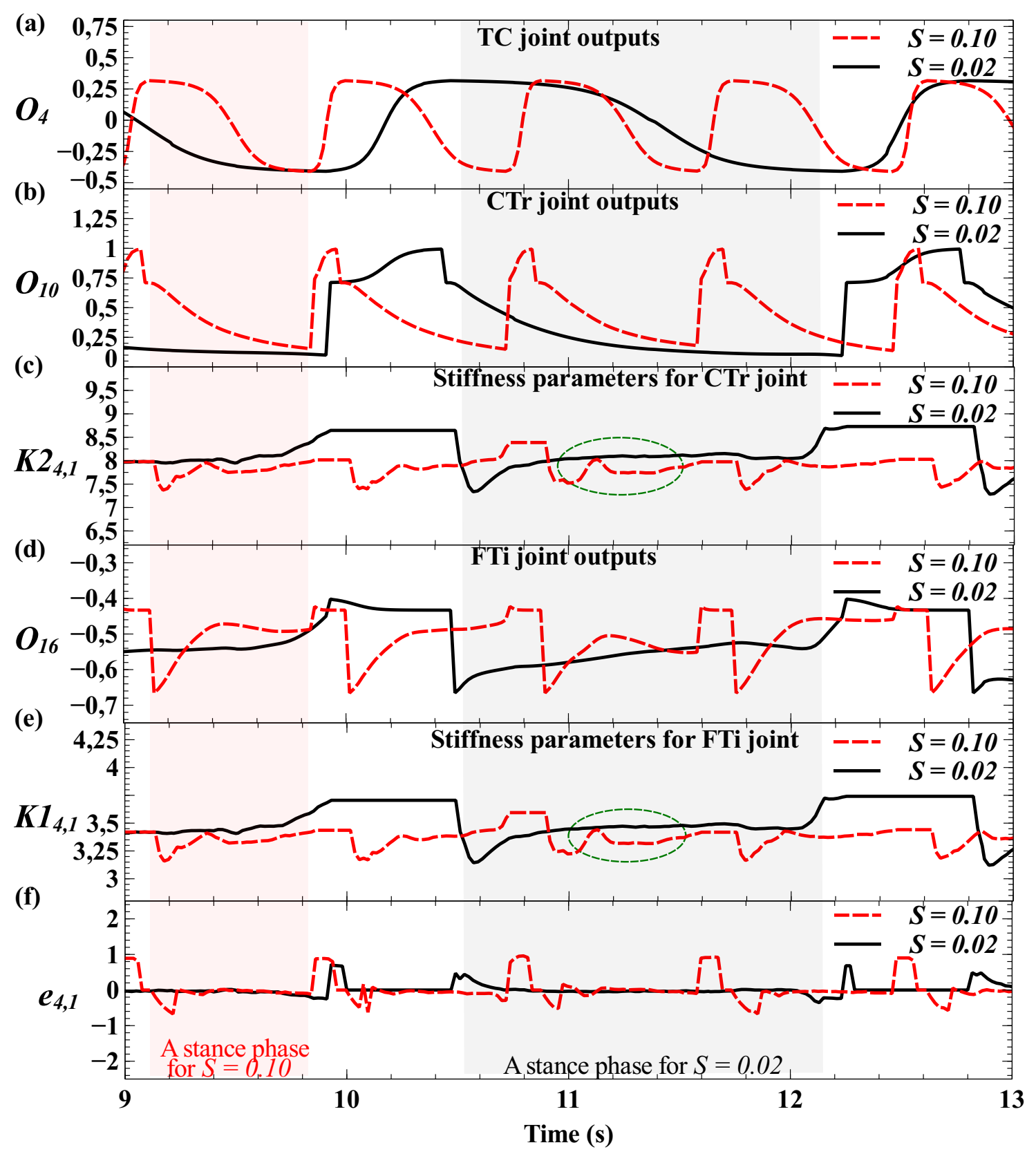

Figure 5.8: Adjustments of $K 1_{4,1}$ and $K 2_{4,1}$ for different gaits. AMOS walked on fine gravel where its gait was chosen as slow wave (i.e., $S=0.02$ ) and fast caterpillar (i.e., $S=0.10$ ) gait, respectively. (a) TC joint outputs $O_{4}$. (b) CTr joint outputs $O_{10}$. (c) Stiffness parameters $K 2_{4,1}$. (d) FTi joint outputs $O_{16}$. (e) Stiffness parameters $K 1_{4,1}$. (f) Foot contact force errors $e_{4,1}$. 
leg motion is increased from the slow gait to the fast one. This finding may reflect a control strategy of polyped (i.e., $>$ two legs) locomotion where legged animals soften the legs owing to the minimization of energetic costs (Spence, 2011). Our experimental results also show that the fast caterpillar gait (i.e., $S=0.10$ ) allows AMOS to achieve softer leg motions, which lead to more energy-efficient locomotion on all experimental surfaces (see costs of transport in Figs. 5.11), compared to the slow wave gait (i.e., $S=0.02$ ). Note that errors during swing phases result from delayed feedback, which makes the phase differences between the real and predicted forces (see Figs. 5.9).

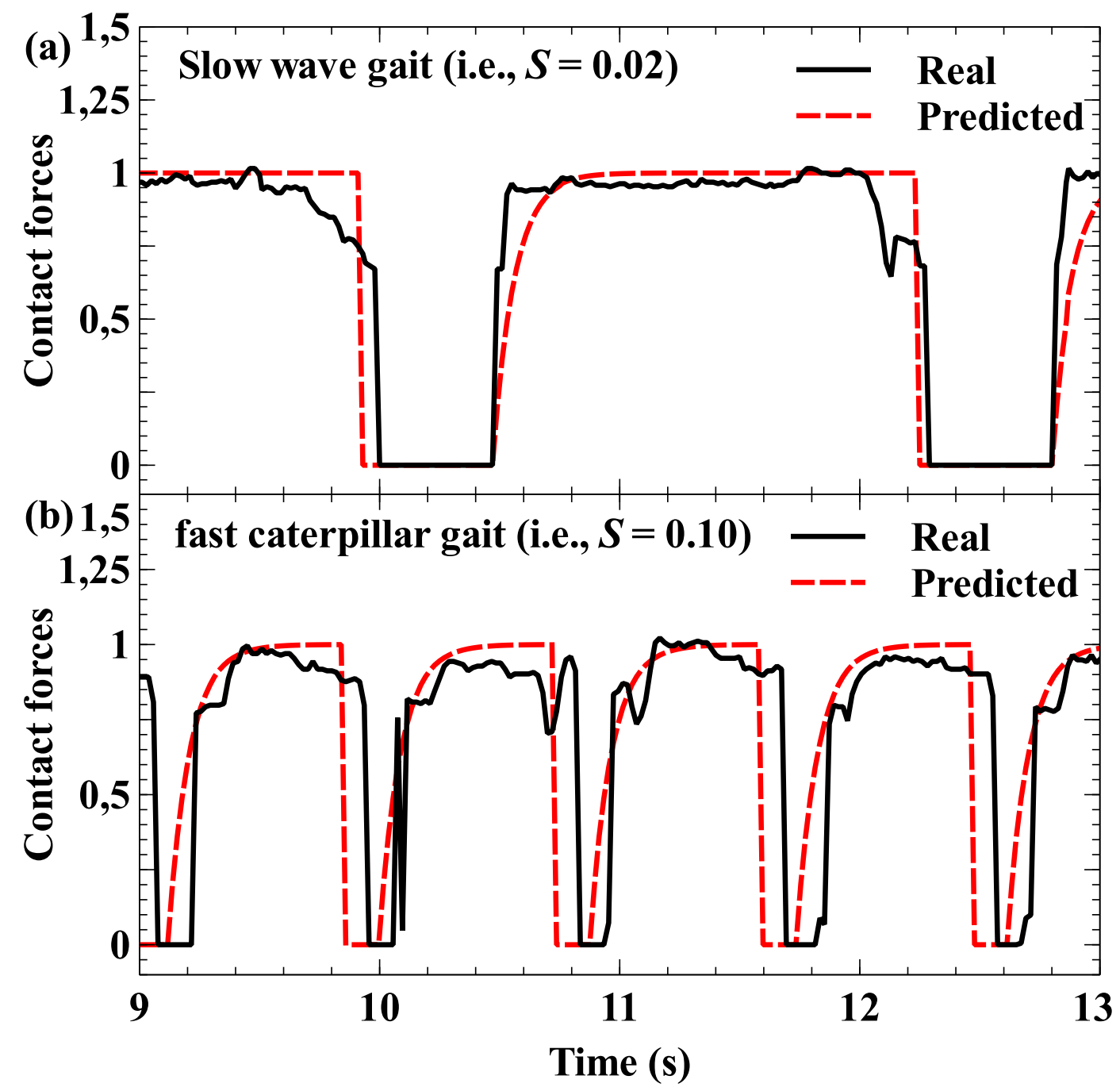

Figure 5.9: Real and predicted contact forces. Here the slow wave (i.e., modulatory input $S=0.02)$ and fast caterpillar $(S=0.10)$ gaits are chosen on fine gravel, respectively. See Figs. 5.8 for more details. 


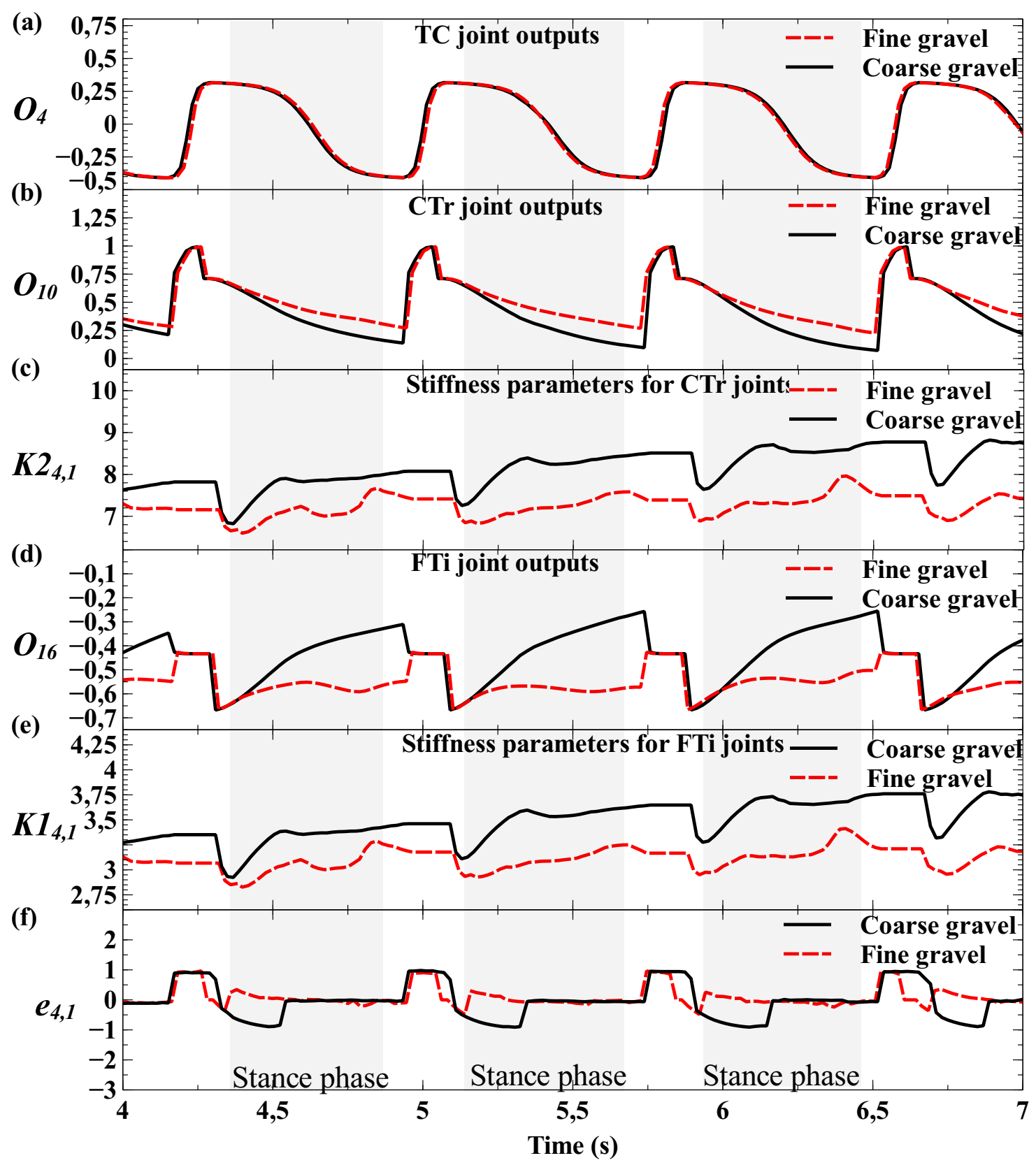

Figure 5.10: Adjustments of $K 1_{4,1}$ and $K 2_{4,1}$ for different surfaces. An intermixed gait (i.e., modulatory input $S=0.12$ ) was chosen for AMOS to walk on fine and coarse gravel, respectively. (a) TC joint outputs $O_{4}$. (b) CTr joint outputs $O_{10}$. (c) Stiffness parameters $K 2_{4,1}$. (d) FTi joint outputs $O_{16}$. (e) Stiffness parameters $K 1_{4,1}$. (f) Foot contact force errors $e_{4,1}$. 


\subsection{Adaptive compliant joint motions for different surfaces}

Actively changing stiffness parameters $K 1_{6 \times 1}$ and $K 2_{6 \times 1}$ also leads to adaptive compliant joint motions on different surfaces. For example, the slow intermixed gait (i.e., modulatory input $S=0.12$ ) was fixed for AMOS to walk on fine and coarse gravel, respectively. On these two surfaces, AMOS joints receive the same motor neuron outputs $^{1}$ of the modular neural network (MNN). One can see that the TC joint motions of the left front leg are the same (see Fig. 5.10 (a)) because they are controlled by only feed-forward neural control (i.e., without passive elements). By contrast, the CTr and FTi joint motions are different (see Figs. 5.10 (b) and (d)) during stance phases when AMOS walks on fine and coarse gravel, respectively. This is because the TC, CTr, and FTi joints act as different roles (i.e., compliance or actuation, see Fig. 4.6 (a)) for controlling leg motions in stance phases. Moreover, we can see that the $\mathrm{CTr}$ and FTi joints are stiffer ${ }^{2}$ (i.e., higher $K 1_{4,1}$ and $K 2_{4,1}$ values, see Figs. 5.10 (c) and (e)) when AMOS walked on coarse gravel, compared to fine gravel. This makes the legs penetrate more deeply, but also extend more widely into the coarse gravel (see CTr and FTi joint motions in Figs. 5.10 (b) and (d)). The video of the experiments can be seen at http://www. youtube.com/watch?v=-Du62APFUt0.

\subsection{The compliant and efficient gaits for walking on the surfaces}

In the previous sections, we show that the adaptive neuromechanical controller enables AMOS to produce adaptive compliant joint motions that accommodate different gaits and surfaces. For each surface, nine gaits (see Figs.5.1) are chosen by changing the modulatory input $S$ (see Eq.(4.2)) of the modular neural network (MNN, see Figs.4.2). The adaptive compliant joint motions lead to different energy efficiencies of AMOS walking on fine gravel, coarse gravel, elastic sponge (stiffness $0.523 \mathrm{kN} / \mathrm{m}$ ), and grass land. Typically, energy efficiency is measured by cost of transport COT (see Eq.(4.12)).

For each gait, we repeatedly ran the hexapod robot on each surface until ten successful runs were obtained. For each successful run, the average power consumption $P_{\text {avg }}$ was calculated based on the electrical current supplied to all motors of AMOS, which is measured by a current sensor. Low COT corresponds to more energy-efficient

\footnotetext{
${ }^{1}$ In our work, the same modulatory input $S$ of the modular neural network (i.e., MNN) corresponds to same motor neuron outputs (i.e., $N_{1-18}$ in Fig. 4.2 (d)).

${ }^{2} \mathrm{~A}$ joint greatly resists the influence of external forces, and is thus "stiff". Whereas, a joint allows external forces to influence its movement easily, and is thus "soft" (Pratt, 2002).
} 

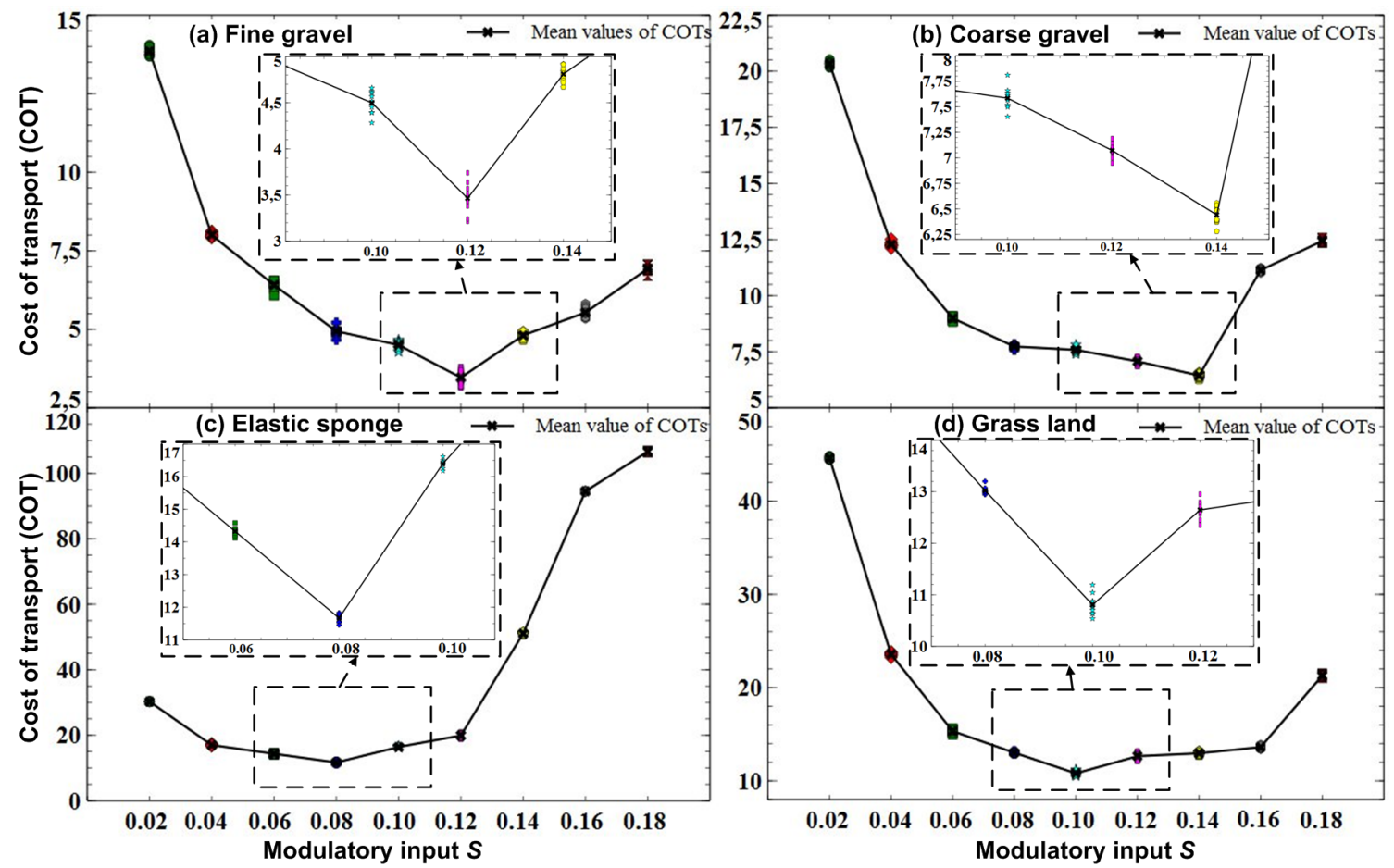

Figure 5.11: Energy efficiencies of AMOS walking on different surfaces using different gaits. (a) COTs on fine gravel. The slow intermixed gait (i.e., $S=0.12$ ) is more efficient. (b) COTs on coarse gravel. The Fast intermixed gait (i.e., $S=0.14$ ) is more efficient. (c) COTs on elastic sponge. The slow caterpillar gait (i.e., $S=0.08$ ) is more efficient. (d) COTs on grass land. The fast caterpillar gait (i.e., $S=0.10$ ) is more efficient.

walking. Figures 5.11 show costs of transport (i.e., COTs) when AMOS walked on the four surfaces using the nine gaits, respectively. One can see that AMOS achieves more energy-efficient walking by using gaits with intermediate leg speeds, compared to a slower leg speed (i.e., modulatory input $S=0.02$, the slow wave gait) or a faster leg speed (i.e., $S=0.18$, the fast tripod gait). Moreover, different gaits make AMOS consume different energetic costs. For instance, the slow intermixed gait (i.e., $S=0.12$ ) enables AMOS to achieve more energy-efficient walking on fine gravel (see Fig. 5.11 (a)) while the fast intermixed gait (i.e., $S=0.14$ ) is an efficient gait for AMOS walking on coarse gravel (see Fig. 5.11 (b)). The slow (i.e., $S=0.08$ ) and fast (i.e., $S=0.10$ ) caterpillar gaits make AMOS achieve more energy-efficient walking on elastic sponge and grass land, respectively (see Figs. 5.11 (c) and (d)). The video of the experiments can be seen at http://www. youtube.com/watch?v=SrasTYQG8Xk. Integrating neuromechanical control and sensorimotor learning, the adaptive neuromechanical controller (see Fig. 5.2) enables AMOS to achieve adaptive compliant 

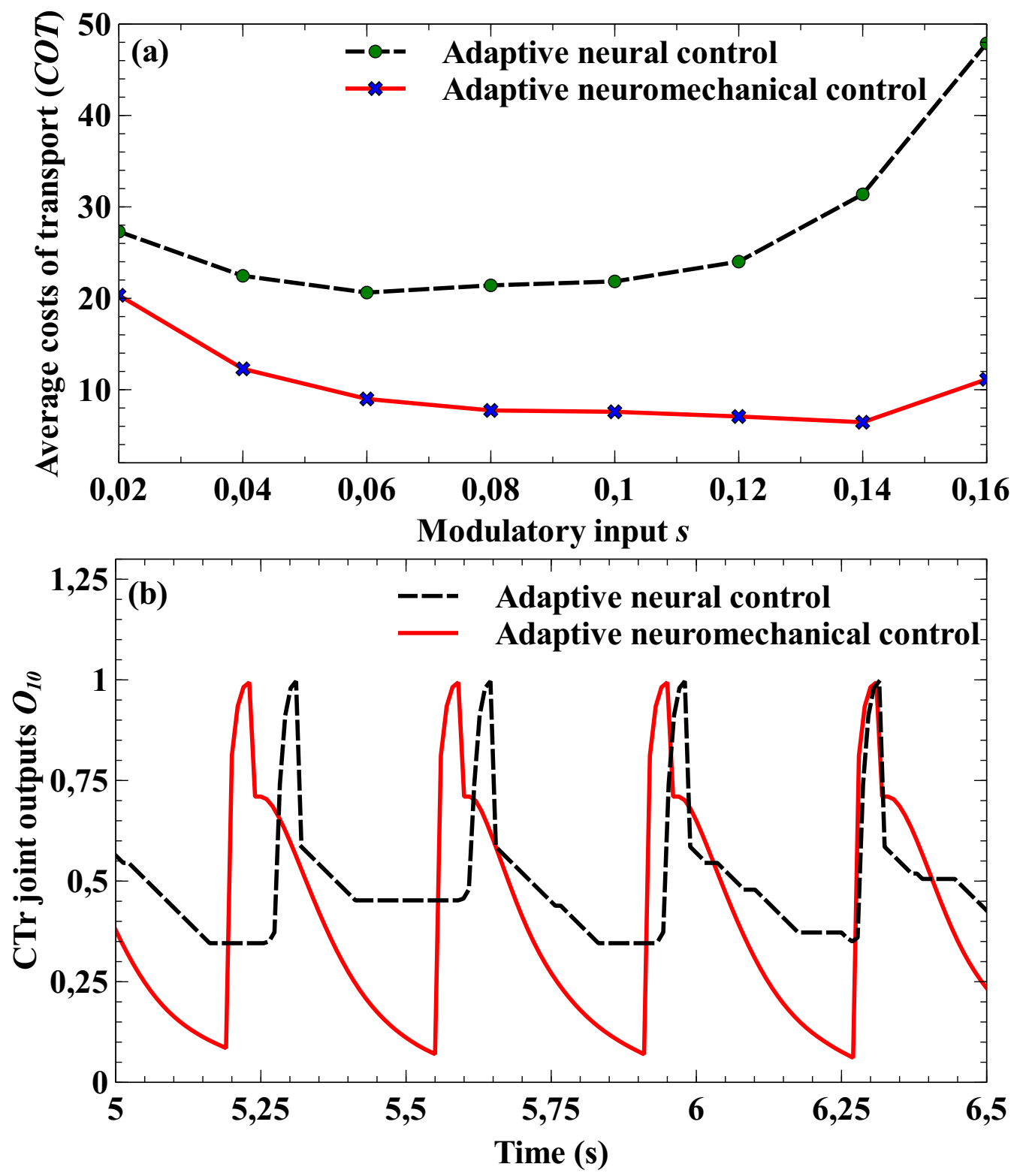

Figure 5.12: Costs of transport COT and CTr joint outputs under the adaptive neuromechanical (see Fig. 5.2) and neural (Manoonpong et al., 2013b) controllers. The experimental surface is coarse gravel. (a) Costs of transports COT. (b) $\mathrm{CTr}$ joint outputs $O_{10}$ (with fast intermixed gaits, modulatory input $S=0.14$ ).

walking, which effectively accommodates different gaits and surfaces. Such walking is achieved by online adjusting stiffness parameters $K 1_{6 \times 1}$ and $K 2_{6 \times 1}$ (see Eqs.(5.3) and (5.4)) of the passive elements (i.e., PEs) driving the FTi and CTr joints. Note that all damper parameters $D(1,2)_{m, 1}$ (see Eqs.(4.5) and (4.8)) are set to 1.0 in 
all experiments chosen by trial and error. As a result, the adaptive neuromechanical controller (see Fig. 5.2) reduces COT of AMOS's walking to between 3.4 and 11.7, compared to mere neuromechanical control (Xiong et al., 2014a). Similarly, the adaptive neuromechanical controller allows for lower $C O T$ that corresponds to more energy-efficient walking (see Fig. 5.12 (a)), compared to the adaptive neural controller (Manoonpong et al., 2013b). This is because the adjustable VAAMs of the adaptive neuromechanical controller produce high amplitude and smooth joint outputs during stance phases (e.g., see Fig. 5.12 (b)), which basically stiffen the legs and allow them to penetrate deeply into challenging surfaces (e.g., coarse gravel). On the other hand, other neural controllers (Manoonpong et al., 2008) like the adaptive neural controller (Manoonpong et al., 2013b) cannot achieve this due to the lack of muscle-like mechanisms (e.g., VAAMs). Furthermore, the adaptive neuromechanical controller enables the insect-like robot AMOS to achieve more energy-efficient walking (see Figs. 5.13), compared to other small legged robots (less than $8 \mathrm{~kg}$ (Galloway, 2010)). However, energy efficiency of AMOS still lags behind that of legged insects (see Figs. 5.13 (f) and $(\mathrm{g}))$, since insect muscles can store and reuse energy.

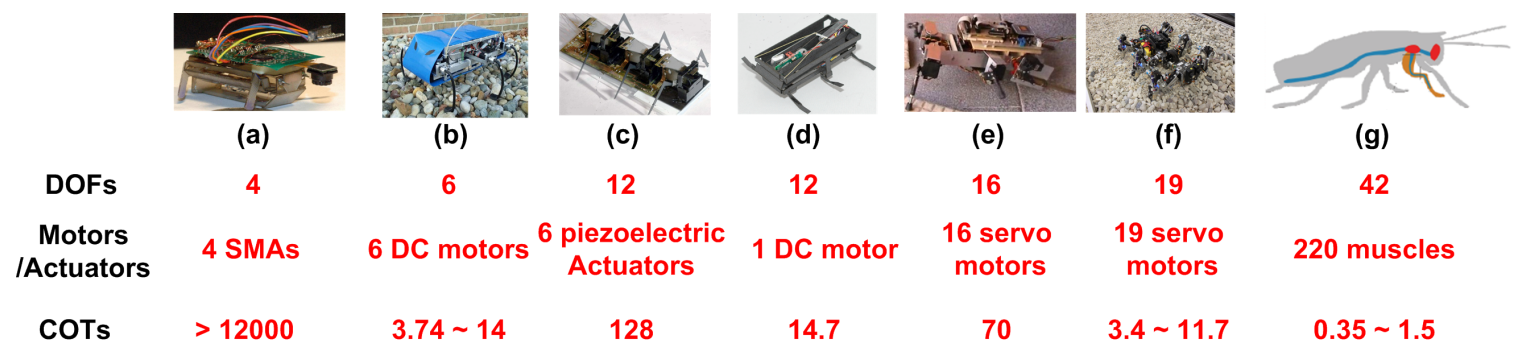

Figure 5.13: Costs of transport (COT) of small legged robots. (a) MEDIC (Kohut et al., 2011). (b) RHex (Saranli et al., 2001). (c) HAMR ${ }^{2}$ (Baisch et al., 2010). (d) DASH (Birkmeyer et al., 2009). (e) Gregor I (Arena et al., 2006). (f) AMOS. (g) Legged insects (Nishii, 2000). 
94 Chapter 5: Adaptive Neuromechanical Control for Online Compliance Adaptation 


\section{6 \\ Conclusions}

\subsection{Summary of contributions}

The motivations and benefits of neuromechanical control on legged robots are in detailed described in (Holmes et al., 2006; Nishikawa et al., 2007; Tytell et al., 2011; Miller et al., 2012) compared to pure neural control or mechanical control (e.g., compliance control). Here, we briefly discuss major methodologies for adaptive and energy-efficient legged robots, since most of the relevant detailed discussions have been provided in the above sections (e.g., see Section 2). In general, the major methodologies have emphasized on neural control (Ijspeert, 2008; Manoonpong et al., 2007), variable compliance control (Semini et al., 2013; Ham et al., 2009; Hurst, 2008) or intelligent mechanical structures (Collins et al., 2005; Ananthanarayanan et al., 2012). Neural control focuses often on emulating behaviors of the nervous systems for coordinated and adaptive legged robots over different surfaces (Manoonpong et al., 2013b; Steingrube et al., 2010). Mere neural control may lead to energy inefficient legged locomotion on different surfaces (Xiong et al., 2014a, 2013), since legged robots cannot achieve variable compliant joint motions. Variable compliance control of legged robots can be achieved by two major methods: active and passive compliance. Typically, active compliance is achieved by software control of joint torques (Boaventura et al., 2013), regardless of additional passive components (e.g., springs). An advantage of active compliance is that the controller enables joint compliance to online adapt for walking on different surfaces. However, the transient responses of the compliant motions intrinsically result in locomotor instabilities, when walking on uneven or rough surfaces (Fukuoka et al., 2003; Garcia and de Santos, 2006). Moreover, adaptive compliance control to a physical legged robot with many DOFs remains an important and unsolved problem in a context of energy-efficient walking on different surfaces (Arevalo and Garcia, 2012; Nishikawa et al., 2007). Specifically, self-adjusting its parameters (i.e., $k_{p}$ and $k_{d}$ in Eq.(2.1)) is a difficult problem in a context of controlling energy-efficient legged locomotion with many DOFs. On the other hand, passive compliance is implemented via hardware that is characterized by physical components such as series elastic actuators (SEAs) (Pratt and Williamson, 1995), artificial muscles (Kingsley et al., 2006), and variable impedance actuators 
(VIAs) (Vanderborght et al., 2013). However, these physical and passive components are still mechanically too bulky and complex for small legged robots (less than $8 \mathrm{~kg}$ (Galloway, 2010)).

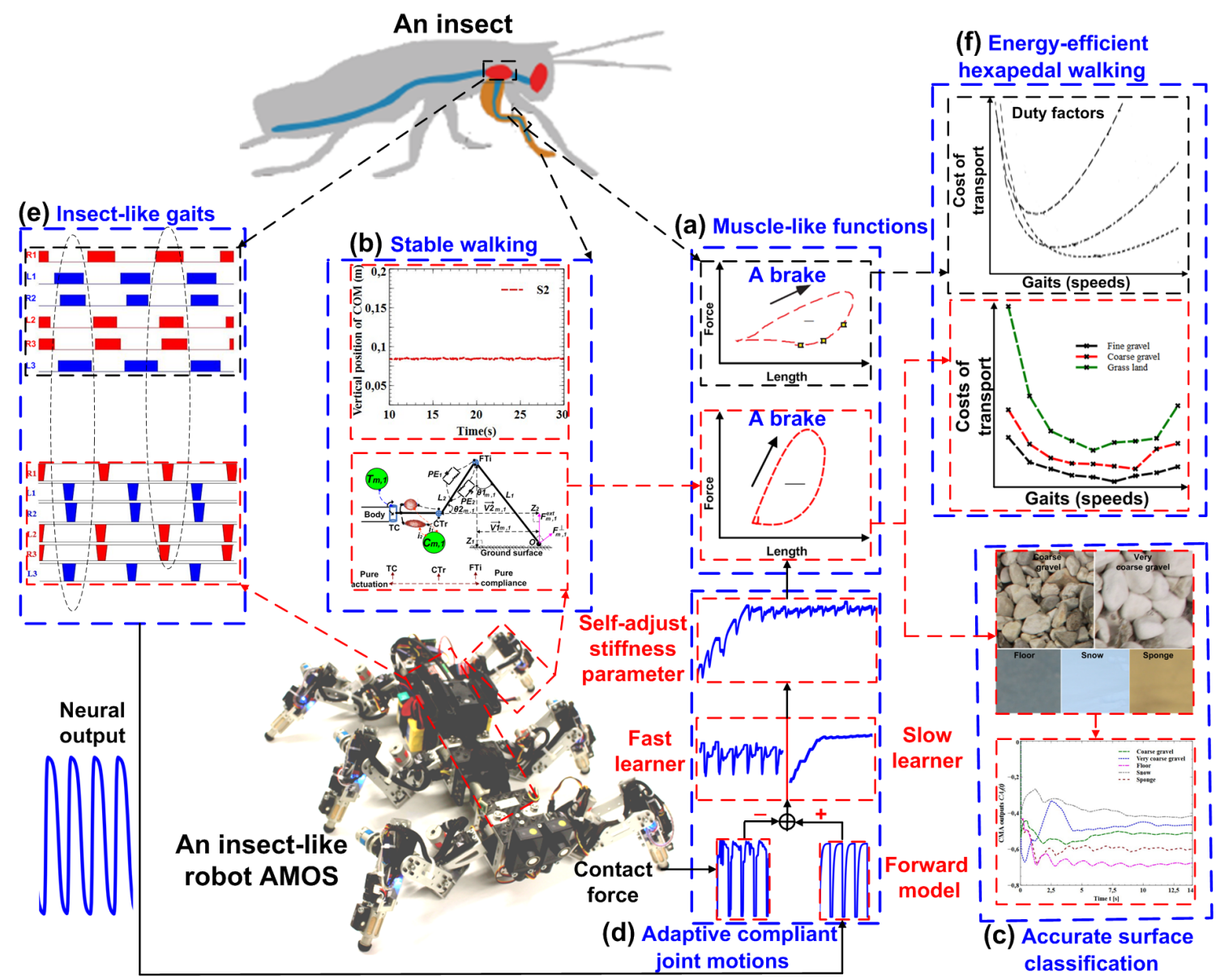

Figure 6.1: The main results of the adaptive neuromechanical controller. (a) Musclelike functions (Dickinson et al., 2000) (e.g., brakes (Ahn and Full, 2002), see Figs. 3.11 (c) and (d)). (b) Stable walking (see Fig. 4.5). (c) Accurate surface (e.g., snow) classification (see Table 4.3). (d) Adaptive compliant joint motions (see Figs. 5.8 and 5.10). (e) Insect-like gaits (see Figs. 5.1, e.g., a tripod gait (Ritzmann and Zill, 2013)). (f) Energy-efficient hexapedal walking (see Figs. 5.11) (Nishii, 2000).

Taken together, compared to other existing methodologies, the main contributions of our method are as follows:

- it develops a simplified muscle model (i.e., the VAAMs, see Fig. 1.2 (b)) for the muscle-like functions that underlie variable compliant joint motions (see Section 3.2); 
- it presents the underlying muscle-like functions (e.g., brakes (Ahn and Full, 2002; Dickinson et al., 2000), see Fig. 6.1 (a)) of the VAAMs that are comparable to those of biological muscles (see Section 3.3);

- it utilizes a proximo-distal gradient of neural control and muscle functions to enhance the stability of legged robot locomotion under adaptive compliance control (see Fig. 6.1 (b) and Chapter 4);

- it achieves variable compliant joint motions relying only on force sensing at the end effectors of the legs. Thus, the implementation reduces sensing and design efforts of legged robots (see Section 4.3);

- it exploits the compliant joint signals generated by the VAAMs to well classify surfaces without using multiple sensing (see Fig. 6.1 (c) and Section 4.4);

- it utilizes sensorimotor learning (see Fig. 6.1 (d)) to self-adjust the stiffness parameters of the VAAMs that adapts to insect-like gaits (e.g., a tripod gait, see Fig. 6.1 (e)) and surfaces (e.g., gravel), thereby leading to more energyefficient hexapedal walking (see Fig. 6.1 (f) and Chapter 5);

- it provides a way forward to model stable and adaptive compliant insect-like walking, by implementing a bio-inspired neural network and several virtual agonist-antagonist mechanisms (VAAMs) on the hexapod robot AMOS with 19 degrees of freedom (i.e., probing Bernstein's 'degrees of freedom' problem (Bernstein, 1967; Nishikawa et al., 2007)) (see Figs. 1.2).

Although the contributions had been made, there are still some unsolved works on neuromechanical control for physical insect-like (i.e., hexapedal) walking. For example, the contractile elements (CEs) of the VAAMs are mathematically approximated setting the length-dependent and velocity-dependent functions to 1 in this thesis. Whereas the two functions should be modeled (see Figs. 3.1) to fit the experimental data obtained from the insects (Ghigliazza and Holmes, 2005; Kukillaya and Holmes, 2009; Tóth et al., 2012). The lengths and velocities of the virtual agonist-antagonist mechanisms (VAAMs) can be derived from additional proprioceptive sensors (e.g., joint position and velocity sensors), which will be added in a new AMOS. As a result, this work will enable the new AMOS to achieve more smooth and insect-like joint motions. In addition, energy efficiency of AMOS still lags behind that of legged insects (see Figs. 5.13 (f) and (g)), since AMOS's morphology (e.g., skin and muscles) isn't optimized yet. Its muscle-like mechanisms (i.e., VAAMs), for instance, fail to store and reuse energy due to their virtual elements, although their applications can reduce structural and sensory complexities of legged robots with many degrees of freedom. Therefore, the VAAMs and physical materials (e.g., shark skin (Manoonpong et al., 2013a)) can be integrated to improve energy-efficient hexapedal walking in future work. 


\subsection{Future work}

The work presented in this thesis aimed to be a fundamental step to model and control physical legged robots in a simplified neuromechanics way. The proposed adaptive neuromechanical controller (see Fig. 5.2) provides a way forward to simplistically model an insect neuromusculoskeletal system by using the modular neural network $(\mathrm{MNN})$, the 18 pairs of the muscle-like mechanisms (VAAMs), and the insect-like robot AMOS with 19 DOFs (degrees of freedom). Whereas the amount of the DOFs is greatly less than that of the muscles driving them in legged animals such as insects. In an insect (e.g., Blaberus discoidalis), for instance, there are 220 muscles controlling its legs with at least 19 DOFs that contribute to its locomotion (Full and Ahn, 1995). In addition, the muscle model adopted in the controller is a simplified insect muscle model (Ghigliazza and Holmes, 2005; Kukillaya and Holmes, 2009; Tóth et al., 2012) where there are only neural activities and lengths that contribute to the active forces generated by the contractile elements (CEs) of the muscle-like mechanisms (i.e., VAAMs). This is because there is no the experimental data obtained from the insects such that the model of the CEs cannot incorporate the length-dependent and velocity-dependent functions. Moreover, such functions are very difficult to be applied to the CEs of the VAAMs driving AMOS due to its lack of the position and velocity sensors at its joints. Similarly, its lack of force/torque sensors at its joints fails to allow adaptive compliant joint motions in the horizontal directions and swing phases. Therefore, the future work based on the presented work can be extended to :

- implement the insect muscle model (Ghigliazza and Holmes, 2005; Kukillaya and Holmes, 2009; Tóth et al., 2012) on AMOS by obtaining experimental data (e.g., muscle force-length data) of insects and adding additional proprioceptive sensors (i.e., joint position and velocity sensors);

- add force/torque sensors at each joint, which allows AMOS to achieve more smooth and adaptive compliant joint motions in the horizontal direction and swing phases;

- increase the amount of the muscle-like mechanisms (i.e., VAAMs) and apply them to AMOS by integrating the insect muscle model and multiple sensory feedback (e.g., force);

- replace the modular neural network (MNN) with the multiple decoupled CPGs (Barikhan et al., 2014) to enable AMOS to online adapt its gaits to energyefficiently walking on different surfaces under the adaptive neuromechanical controller;

- replace the forward models (see Fig. 5.2) with reservoir-based online adaptive forward models (Manoonpong et al., 2014) to accurately predict AMOS's foot contact forces. 
In addition to studying hexapedal locomotion, the adaptive neuromechanical controller can be used to study human-like (i.e., biped) locomotion. With the adaptive neuromechanical controller, biped robots can refer to the adopted strategies of human locomotion to minimize the energetic costs and enhance locomotor stability. For example, the stiffness parameters of the muscle-like mechanisms (i.e., VAAMs) of the controller need to be increased to stiffen the legs of the biped robots that adapts to their walking on the increasingly soft surfaces. This is because humans usually stiffen their legs to improve energy efficiency and enhance locomotor stability on the increasingly soft surfaces (e.g., sponge) (Spence et al., 2010; Farley et al., 1998; Ferris et al., 1998). In return, the biped robots can be good physical platforms to validate and explore the hypotheses of synergistic neuromechanical control of human locomotion by using the adaptive neuromechanical controller. Based on the validated hypotheses, the adaptive neuromechanical controller can be adjusted and applied to the robotic assistive devices (e.g., exoskeletons) to restore the missing leg functions of amputees. 


\section{Bibliography}

Abbas, J. and Full, R. (2000). Neuromechanical interaction in cyclic movements. In Winters, J. and Crago, P., editors, Biomechanics and Neural Control of Posture and Movement, pages 177-191. Springer New York.

Ahn, A. N. (2012). How muscles function the work loop technique. The Journal of Experimental Biology, 215(7):1051-1052.

Ahn, A. N. and Full, R. J. (2002). A motor and a brake: two leg extensor muscles acting at the same joint manage energy differently in a running insect. Journal of Experimental Biology, 205(3):379-389.

Ajallooeian, M., Pouya, S., Sproewitz, A., and Ijspeert, A. (2013). Central pattern generators augmented with virtual model control for quadruped rough terrain locomotion. In Robotics and Automation (ICRA), 2013 IEEE International Conference on, pages 3321-3328.

Albu-Schäffer, A., Eiberger, O., Fuchs, M., Grebenstein, M., Haddadin, S., Ott, C., Stemmer, A., Wimböck, T., Wolf, S., Borst, C., and Hirzinger, G. (2011). Anthropomorphic soft robotics - from torque control to variable intrinsic compliance. In Pradalier, C., Siegwart, R., and Hirzinger, G., editors, The 14th International Symposium ISRR, volume 70 of Springer Tracts in Advanced Robotics, pages 185-207. Springer.

Alexander, R. M. (2003). Principles of Animal Locomotion. Princeton University Press.

Altendorfer, R., Koditschek, D. E., and Holmes, P. (2004). Stability analysis of a clock-driven rigid-body slip model for rhex. The International Journal of Robotics Research, 23(10-11):1001-1012.

Altendorfer, R., Saranli, U., Komsuoglu, H., Koditschek, D., Brown, H.Benjamin, J., Buehler, M., Moore, N., McMordie, D., and Full, R. (2001). Evidence for spring loaded inverted pendulum running in a hexapod robot. In Rus, D. and Singh, S., editors, Experimental Robotics VII, volume 271 of Lecture Notes in Control and Information Sciences, pages 291-302. Springer Berlin Heidelberg.

Ananthanarayanan, A., Azadi, M., and Kim, S. (2012). Towards a bio-inspired leg design for high-speed running. Bioinspiration \& Biomimetics, 7(4):046005.

Arena, P., Fortuna, L., Frasca, M., Patane, L., and Pavone, M. (2006). Realization of a cnn-driven cockroach-inspired robot. In Circuits and Systems, 2006. ISCAS 2006. Proceedings. 2006 IEEE International Symposium on, pages 2649-2652. 
Arevalo, J. and Garcia, E. (2012). Impedance control for legged robots: An insight into the concepts involved. Systems, Man, and Cybernetics, Part C: Applications and Reviews, IEEE Transactions on, 42(6):1400-1411.

Aristotle, Ross, W. D., and Smith, J. A. (1912). The works of Aristotle translated into English. Oxford Clarendon Press.

Arnold, A. S., Lee, D. V., and Biewener, A. A. (2013). Modulation of joint moments and work in the goat hindlimb with locomotor speed and surface grade. The Journal of Experimental Biology.

Baisch, A. T., Sreetharan, P., and Wood, R. (2010). Biologically-inspired locomotion of a $2 \mathrm{~g}$ hexapod robot. In Intelligent Robots and Systems (IROS), 2010 IEEE/RSJ International Conference on, pages 5360-5365.

Barikhan, S., Wörgötter, F., and Manoonpong, P. (2014). Multiple decoupled cpgs with local sensory feedback for adaptive locomotion behaviors of bio-inspired walking robots. In del Pobil, A., Chinellato, E., Martinez-Martin, E., Hallam, J., Cervera, E., and Morales, A., editors, From Animals to Animats 13, volume 8575 of Lecture Notes in Computer Science, pages 65-75. Springer International Publishing.

Bartsch, S., Birnschein, T., Rämmermann, M., Hilljegerdes, J., Kühn, D., and Kirchner, F. (2012). Development of the six-legged walking and climbing robot spaceclimber. Journal of Field Robotics, 29(3):506-532.

Bay, H., Ess, A., Tuytelaars, T., and Gool, L. V. (2008). Speeded-up robust features (surf). Computer Vision and Image Understanding, 110(3):346 - 359. Similarity Matching in Computer Vision and Multimedia.

Bender, J. A., Simpson, E. M., Tietz, B. R., Daltorio, K. A., Quinn, R. D., and Ritzmann, R. E. (2011). Kinematic and behavioral evidence for a distinction between trotting and ambling gaits in the cockroach blaberus discoidalis. The Journal of Experimental Biology, 214(12):2057-2064.

Bermudez, F., Julian, R., Haldane, D., Abbeel, P., and Fearing, R. (2012). Performance analysis and terrain classification for a legged robot over rough terrain. In Intelligent Robots and Systems (IROS), 2012 IEEE/RSJ International Conference on, pages $513-519$.

Bernstein, N. (1967). The coordination and regulation of movements. Oxford : Pergamon Press.

Bhounsule, P. A., Cortell, J., Grewal, A., Hendriksen, B., Karssen, J. G. D., Paul, C., and Ruina, A. (2014). Low-bandwidth reflex-based control for lower power walking: 
$65 \mathrm{~km}$ on a single battery charge. The International Journal of Robotics Research, 33(10):1305-1321.

Biewener, A. A. (2003). Animal Locomotion. Oxford Animol Biology Series. Oxford University Press.

Birkmeyer, P., Peterson, K., and Fearing, R. (2009). Dash: A dynamic 16g hexapedal robot. In Intelligent Robots and Systems, 2009. IROS 2009. IEEE/RSJ International Conference on, pages 2683-2689.

Birnschein, T., Natarajan, G., Bartsch, S., Cordes, F., Kühn, D., and Kirchner, F. (2009). Terrain recognition and environment modeling in legged robots. In Proceedings of the 11th European Regional Conference of the International Society for Terrain-Vehicle Systems. o.A.

Blickhan, R. and Full, R. (1993). Similarity in multilegged locomotion: Bouncing like a monopode. Journal of Comparative Physiology A, 173(5):509-517.

Boaventura, T., Medrano-Cerda, G., Semini, C., Buchli, J., and Caldwell, D. (2013). Stability and performance of the compliance controller of the quadruped robot hyq. In Intelligent Robots and Systems (IROS), 2013 IEEE/RSJ International Conference on, pages 1458-1464.

Borelli, G. A. and Maquet, P. (2014). Borelli's On the Movement of Animals - On the Force of Percussion, volume 37 of Studies in History and Philosophy of Science. Springer.

Bosworth, W., Kim, S., and Hogan, N. (2014). The effect of leg impedance on stability and efficiency in quadrupedal trotting. In Intelligent Robots and Systems (IROS 2014), 2014 IEEE/RSJ International Conference on, pages 4895-4900.

Buchli, J., Stulp, F., Theodorou, E., and Schaal, S. (2011). Learning variable impedance control. The International Journal of Robotics Research.

Büschges, A. (2005). Sensory control and organization of neural networks mediating coordination of multisegmental organs for locomotion. Journal of Neurophysiology, 93(3):1127-1135.

Campos, R., Matos, V., and Santos, C. (2010). Hexapod locomotion: A nonlinear dynamical systems approach. In IECON 2010 - 36th Annual Conference on IEEE Industrial Electronics Society, pages 1546-1551.

Chiel, H. J., Ting, L. H., Ekeberg, Ö., and Hartmann, M. J. Z. (2009). The brain in its body : Motor control and sensing in a biomechanical context. Journal of Neuroscience, 29(41):12807-12814. QC 20100525. 
Collins, S., Ruina, A., Tedrake, R., and Wisse, M. (2005). Efficient bipedal robots based on passive-dynamic walkers. Science, 307(5712):1082-1085.

Daley, M. A. and Biewener, A. A. (2006). Running over rough terrain reveals limb control for intrinsic stability. Proceedings of the National Academy of Sciences, 103(42):15681-15686.

Daley, M. A. and Biewener, A. A. (2011). Leg muscles that mediate stability: mechanics and control of two distal extensor muscles during obstacle negotiation in the guinea fowl. Philosophical Transactions of the Royal Society B: Biological Sciences, 366(1570):1580-1591.

Daley, M. A., Felix, G., and Biewener, A. A. (2007). Running stability is enhanced by a proximo-distal gradient in joint neuromechanical control. Journal of Experimental Biology, 210(3):383-394.

Der, R. and Martius, G. (2012). The lpzrobots simulator. In The Playful Machine, volume 15 of Cognitive Systems Monographs, pages 293-308. Springer Berlin Heidelberg.

Dickinson, M. H., Farley, C. T., Full, R. J., Koehl, M. A. R., Kram, R., and Lehman, S. (2000). How animals move: An integrative view. Science, 288(5463):100-106.

Dillmann, R., Albiez, J., Gaßmann, B., and Kerscher, T. (2005). Biologically motivated control of walking machines. In Climbing and Walking Robots, pages 55-69. Springer Berlin Heidelberg.

Dudek, D. M. and Full, R. J. (2006). Passive mechanical properties of legs from running insects. The Journal of Experimental Biology, 209(8):1502-1515.

Enoch, A., Sutas, A., Nakaoka, S., and Vijayakumar, S. (2012). Blue: A bipedal robot with variable stiffness and damping. In Humanoid Robots (Humanoids), 2012 12th IEEE-RAS International Conference on, pages 487-494.

Ernst, M., Geyer, H., and Blickhan, R. (2012). Extension and customization of self-stability control in compliant legged systems. Bioinspiration 8 Biomimetics, 7(4):046002.

Farahat, W. A. and Herr, H. M. (2010). Optimal workloop energetics of muscle-actuated systems: An impedance matching view. PLoS Comput Biol, 6(6):e1000795.

Farley, C. T., Houdijk, H. H. P., Van Strien, C., and Louie, M. (1998). Mechanism of leg stiffness adjustment for hopping on surfaces of different stiffnesses. Journal of Applied Physiology, 85(3):1044-1055. 
Ferris, D. P., Louie, M., and Farley, C. T. (1998). Running in the real world: adjusting leg stiffness for different surfaces. Proceedings of the Royal Society of London. Series B: Biological Sciences, 265(1400):989-994.

Filitchkin, P. and Byl, K. (2012). Feature-based terrain classification for littledog. In Intelligent Robots and Systems (IROS), 2012 IEEE/RSJ International Conference on, pages 1387-1392.

Fish, F. E. (2002). Balancing requirements for stability and maneuverability in cetaceans. Integrative and Comparative Biology, 42(1):85-93.

Fukuoka, Y., Kimura, H., and Cohen, A. H. (2003). Adaptive dynamic walking of a quadruped robot on irregular terrain based on biological concepts. The International Journal of Robotics Research, 22(3-4):187-202.

Full, R. and Ahn, A. (1995). Static forces and moments generated in the insect leg: comparison of a three-dimensional musculo-skeletal computer model with experimental measurements. The Journal of Experimental Biology, 198(6):1285-1298.

Full, R., Farley, C., and Winters, J. (2000). Musculoskeletal dynamics in rhythmic systems: A comparative approach to legged locomotion. In Winters, J. and Crago, P., editors, Biomechanics and Neural Control of Posture and Movement, pages 192-205. Springer New York.

Full, R. and Koditschek, D. (1999). Templates and anchors: neuromechanical hypotheses of legged locomotion on land. Journal of Experimental Biology, 202(23):3325-3332.

Full, R. J., Kubow, T., Schmitt, J., Holmes, P., and Koditschek, D. (2002). Quantifying dynamic stability and maneuverability in legged locomotion. Integrative and Comparative Biology, 42(1):149-157.

Full, R. J., Stokes, D. R., Ahn, A. N., and Josephson, R. K. (1998). Energy absorption during running by leg muscles in a cockroach. Journal of Experimental Biology, 201(7):997-1012.

Gabaldón, A. M., Nelson, F. E., and Roberts, T. J. (2004). Mechanical function of two ankle extensors in wild turkeys: shifts from energy production to energy absorption during incline versus decline running. Journal of Experimental Biology, 207(13):2277-2288.

Galloway, K., Clark, J., Yim, M., and Koditschek, D. (2011). Experimental investigations into the role of passive variable compliant legs for dynamic robotic locomotion. In Robotics and Automation (ICRA), 2011 IEEE International Conference on, pages $1243-1249$. 
Galloway, K. C. (2010). Passive Variable Compliance for Dynamic Legged Robots. Phd thesis, University of Pennsylvania.

Galloway, K. C., Clark, J. E., and Koditschek, D. E. (2013). Variable stiffness legs for robust, efficient, and stable dynamic running. Journal of Mechanisms and Robotics, 5:011009(11).

Garcia, E. and de Santos, P. (2006). On the improvement of walking performance in natural environments by a compliant adaptive gait. Robotics, IEEE Transactions on, 22(6):1240-1253.

Ghigliazza, R. M. and Holmes, P. (2005). Towards a neuromechanical model for insect locomotion: hybrid dynamical systems. REGUL. CHAOTIC DYN., 10:193-225.

Giguere, P., Dudek, G., Saunderson, S., and Prahacs, C. (2006). Environment identification for a running robot using inertial and actuator cues. In Proceedings of Robotics: Science and Systems, Philadelphia, USA.

Goldschmidt, D., Wörgötter, F., and Manoonpong, P. (2014). Biologically-inspired adaptive obstacle negotiation behavior of hexapod robots. Frontiers in Neurorobotics, 8(3).

Gregorio, P., Ahmadi, M., and Buehler, M. (1997). Design, control, and energetics of an electrically actuated legged robot. Systems, Man, and Cybernetics, Part B: Cybernetics, IEEE Transactions on, 27(4):626 -634.

Haeufle, D. F. B., Grimmer, S., and Seyfarth, A. (2010). The role of intrinsic muscle properties for stable hoppingstability is achieved by the forcevelocity relation. Bioinspiration $\& 3$ Biomimetics, 5(1):016004.

Ham, R., Sugar, T., Vanderborght, B., Hollander, K., and Lefeber, D. (2009). Compliant actuator designs. Robotics Automation Magazine, IEEE, 16(3):81 -94.

Heitmann, S., Breakspear, M., and Ferns, N. (2012). Muscle co-contraction modulates damping and joint stability in a three-link bio mechanical limb. Frontiers in Neurorobotics, 5(5).

Hill, A. V. (1938). The heat of shortening and the dynamic constants of muscle. Proceedings of the Royal Society of London. Series B - Biological Sciences, 126(843):136-195.

Hoepflinger, M., Remy, C., Hutter, M., Spinello, L., and Siegwart, R. (2010). Haptic terrain classification for legged robots. In Robotics and Automation (ICRA), 2010 IEEE International Conference on, pages 2828-2833. 
Hogan, N. and Buerger, S. P. (2004). Impedance and Interaction Control, volume Chapter 19 of Robotics and Automation Handbook. CRC Press.

Hollander, K., Sugar, T., and Herring, D. (2005). Adjustable robotic tendon using a 'jack spring';. In Rehabilitation Robotics, 2005. ICORR 2005. 9th International Conference on, pages 113-118.

Holmes, P., Full, R., Koditschek, D., and Guckenheimer, J. (2006). The dynamics of legged locomotion: Models, analyses, and challenges. SIAM Review, 48(2):207-304.

Hughes, G. M. (1952). The co-ordination of insect movements i the walking movements of insects. J. Exp. Biol., 29:267-285.

Hurst, J., Chestnutt, J., and Rizzi, A. (2010). The actuator with mechanically adjustable series compliance. Robotics, IEEE Transactions on, 26(4):597 -606.

Hurst, J. W. (2008). The Role and Implementation of Compliance in Legged Locomotion. PhD thesis, Robotics Institute, Carnegie Mellon University, Pittsburgh, PA.

Hutter, M., Hoepflinger, M., Gehring, C., Bloesch, M., Remy, C. D., and Siegwart, R. (2012). Hybrid operational space control for compliant legged systems. In Proceedings of Robotics: Science and Systems, Sydney, Australia.

Hutter, M., Remy, C., Hoepflinger, M., and Siegwart, R. (2011). Scarleth: Design and control of a planar running robot. In Intelligent Robots and Systems (IROS), 2011 IEEE/RSJ International Conference on, pages 562-567.

Hwangbo, J., Gehring, C., Siegwart, R., and Buchli, J. (2014). Variable impedance control for legged robots. In Dynamic Walking.

Hyun, D. J., Seok, S., Lee, J., and Kim, S. (2014). High speed trot-running: Implementation of a hierarchical controller using proprioceptive impedance control on the mit cheetah. The International Journal of Robotics Research.

I. Havoutis, C. Semini, J. B. and Caldwell, D. (2012). Progress in quadrupedal trotting with active compliance. In Dynamic Walking 2012, Pensacola, Florida, USA.

Iijima, H., Sayama, K., Masuta, H., Takanishi, A., and ok Lim, H. (2013). Mechanism of one-legged jumping robot with artificial musculoskeletal system. In Control, Automation and Systems (ICCAS), 2013 13th International Conference on, pages 869-874.

Ijspeert, A. J. (2008). Central pattern generators for locomotion control in animals and robots: A review. Neural Networks, 21(4):642 - 653. Robotics and Neuroscience. 
Jacob Shill, Emmanuel Collins, J. C. (2012). Tactile terrain classification technique for legged robots. In Dynamic Walking. Pensacola, Florida., Dynamic Walking Conference.

Johnson, A. M., Haynes, G. C., and Koditschek, D. E. (2010). Disturbance detection, identification, and recovery by gait transition in legged robots. In Proceedings of the IEEE/RSJ Intl. Conference on Intelligent Robots and Systems, pages 5347-5353, Taipei, Taiwan.

Kaliyamoorthy, S., Quinn, R. D., and Zill, S. N. (2005). Force sensors in hexapod locomotion. The International Journal of Robotics Research, 24(7):563-574.

Kar, D., Issac, K. K., and Jayarajan, K. (2003). Gaits and energetics in terrestrial legged locomotion. Mechanism and Machine Theory, 38(4):355 - 366. NaCOMM99, the National Conference on Machines and Mechanisms.

Kerdok, A. E., Biewener, A. A., McMahon, T. A., Weyand, P. G., and Herr, H. M. (2002). Energetics and mechanics of human running on surfaces of different stiffnesses. Journal of Applied Physiology, 92(2):469-478.

Kingsley, D., Quinn, R., and Ritzmann, R. E. (2006). A cockroach inspired robot with artificial muscles. In Intelligent Robots and Systems, 2006 IEEE/RSJ International Conference on, pages 1837-1842.

Knight, K. (2011). Cockroach trot and amble with tripod gaits. The Journal of Experimental Biology, 214(12):i-ii.

Koditschek, D. E., Full, R. J., and Buehler, M. (2004). Mechanical aspects of legged locomotion control. Arthropod Structure and Development, 33(3):251 - 272. Arthropod Locomotion Systems: from Biological Materials and Systems to Robotics.

Kohut, N., Hoover, A., Ma, K., Baek, S., and Fearing, R. (2011). Medic: A legged millirobot utilizing novel obstacle traversal. In Robotics and Automation (ICRA), 2011 IEEE International Conference on, pages 802-808.

Koos, S., Cully, A., and Mouret, J.-B. (2013). Fast damage recovery in robotics with the t-resilience algorithm. The International Journal of Robotics Research, 32(14):1700-1723.

Kukillaya, R., Proctor, J., and Holmes, P. (2009). Neuromechanical models for insect locomotion: Stability, maneuverability, and proprioceptive feedback. Chaos: An Interdisciplinary Journal of Nonlinear Science, 19(2).

Kukillaya, R. P. and Holmes, P. (2009). A model for insect locomotion in the horizontal plane: Feedforward activation of fast muscles, stability, and robustness. Journal of Theoretical Biology, 261(2):210 - 226. 
Laffranchi, M., Tsagarakis, N., and Caldwell, D. (2011). A compact compliant actuator (compact) with variable physical damping. In Robotics and Automation (ICRA), 2011 IEEE International Conference on, pages $4644-4650$.

Lappin, A. K., Monroy, J. A., Pilarski, J. Q., Zepnewski, E. D., Pierotti, D. J., and Nishikawa, K. C. (2006). Storage and recovery of elastic potential energy powers ballistic prey capture in toads. Journal of Experimental Biology, 209(13):2535-2553.

Lee, D. V. and Biewener, A. A. (2011). Bigdog-inspired studies in the locomotion of goats and dogs. Integrative and Comparative Biology, 51(1):190-202.

Lee, D. V., McGuigan, M. P., Yoo, E. H., and Biewener, A. A. (January 2008a). Compliance, actuation, and work characteristics of the goat foreleg and hindleg during level, uphill, and downhill running. Journal of Applied Physiology, 104(1):130-141.

Lee, J., Sponberg, S., Loh, O., Lamperski, A., Full, R., and Cowan, N. (2008b). Templates and anchors for antenna-based wall following in cockroaches and robots. Robotics, IEEE Transactions on, 24(1):130-143.

Luca, A. D., Siciliano, B., and Zollo, L. (2005). \{PD $\}$ control with on-line gravity compensation for robots with elastic joints: Theory and experiments. Automatica, 41(10):1809 - 1819 .

Manoonpong, P., Dasgupta, S., Goldschmidt, D., and Wörgötter, F. (2014). Reservoir-based online adaptive forward models with neural control for complex locomotion in a hexapod robot. In Neural Networks (IJCNN), 2014 International Joint Conference on, pages 3295-3302.

Manoonpong, P., Goldschmidt, D., Wörgötter, F., Kovalev, A., Heepe, L., and Gorb, S. (2013a). Using a biological material to improve locomotion of hexapod robots. In Lepora, N., Mura, A., Krapp, H., Verschure, P., and Prescott, T., editors, Biomimetic and Biohybrid Systems, volume 8064 of Lecture Notes in Computer Science, pages 402-404. Springer Berlin Heidelberg.

Manoonpong, P., Parlitz, U., and Wörgötter, F. (2013b). Neural control and adaptive neural forward models for insect-like, energy-efficient, and adaptable locomotion of walking machines. Frontiers in Neural Circuits, 7(12).

Manoonpong, P., Pasemann, F., and Roth, H. (2007). Modular reactive neurocontrol for biologically inspired walking machines. The International Journal of Robotics Research, 26(3):301-331.

Manoonpong, P., Pasemann, F., and Wörgötter, F. (2008). Sensor-driven neural control for omnidirectional locomotion and versatile reactive behaviors of walking machines. Robotics and Autonomous Systems, 56(3):265 - 288. 
Manoonpong, P. and Wörgötter, F. (2009). Efference copies in neural control of dynamic biped walking. Robotics and Autonomous Systems, 57(11):1140 - 1153.

Mario, T. (2008). Da Vinci 's Robots. New mechanics and new automata found in codices. Ed. Leonardo3.

McCrea, D. A. and Rybak, I. A. (2008). Organization of mammalian locomotor rhythm and pattern generation. Brain Research Reviews, 57(1):134 - 146.

McMahon, T. A. and Cheng, G. C. (1990). The mechanics of running: How does stiffness couple with speed? Journal of Biomechanics, 23, Supplement 1(0):65 78. International Society of Biomechanics.

McNichol, T. and Full, R. J. (2002). Why 6-Legged Bots Rule. Wired.com.

Miller, L. A., Goldman, D. I., Hedrick, T. L., Tytell, E. D., Wang, Z. J., Yen, J., and Alben, S. (2012). Using computational and mechanical models to study animal locomotion. Integrative and Comparative Biology, 52(5):553-575.

Nagle, J. (1995a). Realistic animation of legged running on rough terrain. In Computer Animation '95., Proceedings., pages 154-162, 216.

Nagle, J. (1995b). Why legs have three joints. Unpublished work.

Nishi, J. (1998). Gait pattern and energetic cost in hexapods. In Engineering in Medicine and Biology Society, 1998. Proceedings of the 20th Annual International Conference of the IEEE, volume 5, pages 2430-2433 vol.5.

Nishii, J. (2000). Legged insects select the optimal locomotor pattern based on the energetic cost. Biological Cybernetics, 83(5):435-442.

Nishikawa, K., Biewener, A. A., Aerts, P., Ahn, A. N., Chiel, H. J., Daley, M. A., Daniel, T. L., Full, R. J., Hale, M. E., Hedrick, T. L., Lappin, A. K., Nichols, T. R., Quinn, R. D., Satterlie, R. A., and Szymik, B. (2007). Neuromechanics: an integrative approach for understanding motor control. Integrative and Comparative Biology, 47(1):16-54.

Oku, T., Inoue, K., Pham, T., Tominaga, K., Maeda, D., Uemura, M., Hirai, H., and Miyazaki, F. (2012). Analysis of muscle coordination in human pedaling and implementation with a musculoskeletal robot. In Humanoid Robots (Humanoids), 2012 12th IEEE-RAS International Conference on, pages 606-611.

Ott, C., Mukherjee, R., and Nakamura, Y. (2010). Unified impedance and admittance control. In Robotics and Automation (ICRA), 2010 IEEE International Conference on, pages $554-561$. 
Pasemann, F., Hild, M., and Zahedi, K. (2003). So(2)-networks as neural oscillators. In Mira, J. and Álvarez, J., editors, Computational Methods in Neural Modeling, volume 2686 of Lecture Notes in Computer Science, pages 144-151. Springer Berlin Heidelberg.

Pratt, G. (2000). Legged robots at mit: what's new since raibert? Robotics Automation Magazine, IEEE, 7(3):15-19.

Pratt, G. and Williamson, M. (1995). Series elastic actuators. In Intelligent Robots and Systems 95. 'Human Robot Interaction and Cooperative Robots', Proceedings. 1995 IEEE/RSJ International Conference on, volume 1, pages 399-406 vol.1.

Pratt, G. A. (2002). Low impedance walking robots. Integrative and Comparative Biology, 42(1):174-181.

Pratt, J., Chew, C.-M., Torres, A., Dilworth, P., and Pratt, G. (2001). Virtual model control: An intuitive approach for bipedal locomotion. The International Journal of Robotics Research, 20(2):129-143.

Pratt, J., Krupp, B., and Morse, C. (2002). Series elastic actuators for high fidelity force control. Industrial Robot: An International Journal, 29(3):234 - 241.

Proctor, J. and Holmes, P. (2010). Reflexes and preflexes: on the role of sensory feedback on rhythmic patterns in insect locomotion. Biological Cybernetics, 102(6):513531.

Raibert, M., Blankespoor, K., Nelson, G., Playter, R., and the BigDog Team (2008). Bigdog, the rough-terrain quadruped robot. In Chung, M. J., editor, Proceedings of the 17th IFAC World Congress, 2008, volume 17, pages 10822-10825.

Raibert, M. H. and Hodgins, J. K. (1991). Animation of dynamic legged locomotion. In Proceedings of the 18th Annual Conference on Computer Graphics and Interactive Techniques, SIGGRAPH '91, pages 349-358, New York, NY, USA. ACM.

Revzen, S., Burden, S., Moore, T., Mongeau, J.-M., and Full, R. (2013). Instantaneous kinematic phase reflects neuromechanical response to lateral perturbations of running cockroaches. Biological Cybernetics, 107(2):179-200.

Revzen, S., Koditschek, D., and Full, R. (2009). Towards testable neuromechanical control architectures for running. In Sternad, D., editor, Progress in Motor Control, volume 629 of Advances in Experimental Medicine and Biology, pages 25-55. Springer US.

Ritzmann, R. and Zill, S. N. (2013). Neuroethology of insect walking. In Scholarpedia. Encyclopedia. 
Roberts, T. J., Marsh, R. L., Weyand, P. G., and Taylor, C. R. (1997). Muscular force in running turkeys: The economy of minimizing work. Science, 275(5303):11131115 .

Rönnau, A., Heppner, G., Nowicki, M., and Dillmann, R. (2014). Lauron v: A versatile six-legged walking robot with advanced maneuverability. In Advanced Intelligent Mechatronics (AIM), 2014 IEEE/ASME International Conference on, pages $82-87$.

Rosendo, A., Nakatsu, S., Narioka, K., and Hosoda, K. (2013). Pneupard: A biomimetic musculoskeletal approach for a feline-inspired quadruped robot. In Intelligent Robots and Systems (IROS), 2013 IEEE/RSJ International Conference on, pages $1452-1457$.

Roth, E., Sponberg, S., and Cowan, N. (2014). A comparative approach to closedloop computation. Current Opinion in Neurobiology, 25(0):54 - 62. Theoretical and computational neuroscience.

Saranli, U., Buehler, M., and Koditschek, D. E. (2001). Rhex: A simple and highly mobile hexapod robot. The International Journal of Robotics Research, 20(7):616631.

Schmidt, A. and Walas, K. (2013). The classification of the terrain by a hexapod robot. In Burduk, R., Jackowski, K., Kurzynski, M., Wozniak, M., and Zolnierek, A., editors, Proceedings of the 8th International Conference on Computer Recognition Systems CORES 2013, volume 226 of Advances in Intelligent Systems and Computing, pages 825-833. Springer International Publishing.

Schmitt, S., Haeufle, D. F. B., Blickhan, R., and Günther, M. (2012). Nature as an engineer: one simple concept of a bio-inspired functional artificial muscle. Bioinspiration $\&$ Biomimetics, 7(3):036022.

Seipel, J. (2005). The stability of point-mass hoppers with varying morphology and minimal feedback. In Proceedings of Robotics: Science and Systems, Cambridge, USA.

Semini, C., Barasuol, V., Boaventura, T., Frigerio, M., and Buchli, J. (2013). Is active impedance the key to a breakthrough for legged robots? In International Symposium of Robotics Research (ISRR).

Semini, C., Tsagarakis, N. G., Guglielmino, E., Focchi, M., Cannella, F., and Caldwell, D. G. (2011). Design of hyq a hydraulically and electrically actuated quadruped robot. Proceedings of the Institution of Mechanical Engineers, Part I: Journal of Systems and Control Engineering, 225(6):831-849. 
Sensinger, J. (2010). Variable admittance actuators using series elasticity. In Robotics and Automation (ICRA), 2010 IEEE International Conference on, VIA Workshop.

Sentis, L., Park, J., and Khatib, O. (2010). Compliant control of multicontact and center-of-mass behaviors in humanoid robots. Robotics, IEEE Transactions on, 26(3):483-501.

Seyfarth, A., Geyer, H., Blickhan, R., Lipfert, S., Rummel, J., Minekawa, Y., and Iida, F. (2006). Running and walking with compliant legs. In Diehl, M. and Mombaur, K., editors, Fast Motions in Biomechanics and Robotics, volume 340 of Lecture Notes in Control and Information Sciences, pages 383-401. Springer Berlin Heidelberg.

Shadmehr, R. and Wise, S. P. (2005). The Computational Neurobiology of Reaching and Pointing. MIT Press.

Smith, M. A., Ghazizadeh, A., and Shadmehr, R. (2006). Interacting adaptive processes with different timescales underlie short-term motor learning. PLoS Biol, 4(6):e179.

Soltero, D., Julian, B., Onal, C., and Rus, D. (2013). A lightweight modular 12-dof print-and-fold hexapod. In Intelligent Robots and Systems (IROS), 2013 IEEE/RSJ International Conference on, pages 1465-1471.

Spence, A. J. (2009). Scaling in biology. Current Biology, 19(2):R57 - R61.

Spence, A. J. (2011). Control strategies for legged locomotion: a comparative approach. In 7th European Nonlinear Dynamics Conference (ENOC), Rome, Italy.

Spence, A. J., Revzen, S., Seipel, J., Mullens, C., and Full, R. J. (2010). Insects running on elastic surfaces. The Journal of Experimental Biology, 213(11):19071920.

Sponberg, S. and Full, R. J. (2008). Neuromechanical response of musculo-skeletal structures in cockroaches during rapid running on rough terrain. Journal of Experimental Biology, 211(3):433-446.

Sponberg, S., Libby, T., Mullens, C. H., and Full, R. J. (2011). Shifts in a single muscle's control potential of body dynamics are determined by mechanical feedback. Philosophical Transactions of the Royal Society B: Biological Sciences, 366(1570):1606-1620.

Sreenath, K., Park, H.-W., Poulakakis, I., and Grizzle, J. (2010). Design and experimental implementation of a compliant hybrid zero dynamics controller for walking on mabel. In Decision and Control (CDC), 2010 49th IEEE Conference on, pages $280-287$. 
Steingrube, S., Timme, M., Worgotter, F., and Manoonpong, P. (2010). Self-organized adaptation of a simple neural circuit enables complex robot behaviour. Nat Phys, 6(3):224-230.

Stephens, B. and Atkeson, C. (2010a). Dynamic balance force control for compliant humanoid robots. In Intelligent Robots and Systems (IROS), 2010 IEEE/RSJ International Conference on, pages 1248-1255.

Stephens, B. and Atkeson, C. (2010b). Push recovery by stepping for humanoid robots with force controlled joints. In Humanoid Robots (Humanoids), 2010 10th IEEE-RAS International Conference on, pages $52-59$.

Ting, L. H., van Antwerp, K. W., Scrivens, J. E., McKay, J. L., Welch, T. D. J., Bingham, J. T., and DeWeerth, S. P. (2009). Neuromechanical tuning of nonlinear postural control dynamics. Chaos: An Interdisciplinary Journal of Nonlinear Science, 19(2).

Tomaž, K. (2004). Stability in legged locomotion. Biological Cybernetics, 90(1):51-58.

Tomei, P. (1991). Adaptive pd controller for robot manipulators. Robotics and Automation, IEEE Transactions on, 7(4):565-570.

Tóth, T. I., Knops, S., and Daun-Gruhn, S. (2012). A neuromechanical model explaining forward and backward stepping in the stick insect. Journal of Neurophysiology, 107(12):3267-3280.

Tsujita, K., Kobayashi, T., Inoura, T., and Masuda, T. (2008). Gait transition by tuning muscle tones using pneumatic actuators in quadruped locomotion. In Intelligent Robots and Systems, 2008. IROS 2008. IEEE/RSJ International Conference on, pages 2453-2458.

Tsujita, K. and Miki, K. (2011). A study on trunk stiffness and gait stability in quadrupedal locomotion using musculoskeletal robot. In Advanced Robotics (ICAR), 2011 15th International Conference on, pages 316-321.

Tytell, E., Holmes, P., and Cohen, A. (2011). Spikes alone do not behavior make: why neuroscience needs biomechanics. Current Opinion in Neurobiology, 21(5):816 - 822. Networks, circuits and computation.

Vanderborght, B., Albu-Schaeffer, A., Bicchi, A., Burdet, E., Caldwell, D., Carloni, R., Catalano, M., Eiberger, O., Friedl, W., Ganesh, G., Garabini, M., Grebenstein, M., Grioli, G., Haddadin, S., Hoppner, H., Jafari, A., Laffranchi, M., Lefeber, D., Petit, F., Stramigioli, S., Tsagarakis, N., Damme, M. V., Ham, R. V., Visser, L., and Wolf, S. (2013). Variable impedance actuators: A review. Robotics and Autonomous Systems, 61(12):1601 - 1614. 
Vanderborght, B., Tsagarakis, N., Van Ham, R., Thorson, I., and Caldwell, D. (2011). Maccepa 2.0: compliant actuator used for energy efficient hopping robot chobino1d. Autonomous Robots, 31(1):55-65.

Vanderborght, B., Van Ham, R., Verrelst, B., Van Damme, M., and Lefeber, D. (2008). Overview of the lucy project: Dynamic stabilization of a biped powered by pneumatic artificial muscles. Advanced Robotics, 22(10):1027-1051.

Wampler, K. and Popović, Z. (2009). Optimal gait and form for animal locomotion. ACM Trans. Graph., 28(3):60:1-60:8.

Webb, B. (2004). Neural mechanisms for prediction: do insects have forward models? Trends in Neurosciences, 27(5):278 - 282.

Winkler, A., Havoutis, I., Bazeille, S., Ortiz, J., Focchi, M., Dillmann, R., Caldwell, D., and Semini, C. (2014). Path planning with force-based foothold adaptation and virtual model control for torque controlled quadruped robots. In Robotics and Automation (ICRA), 2014 IEEE International Conference on, pages 6476-6482.

Wolpert, D. M., Diedrichsen, J., and Flanagan, J. R. (2011). Principles of sensorimotor learning. Nat Rev Neurosci, 12(12):739-751.

Xiong, X., Wörgötter, F., and Manoonpong, P. (2012). An adaptive neuromechanical model for muscle impedance modulations of legged robots. In Dynamic Walking Conference.

Xiong, X., Wörgötter, F., and Manoonpong, P. (2013). A neuromechanical controller of a hexapod robot for walking on sponge, gravel and snow surfaces. In Advances in Artificial Life. Proceedings of the 11th European Conference on Artificial Life ECAL 2013, pages 989-996, Taormina, Italy.

Xiong, X., Wörgötter, F., and Manoonpong, P. (2014a). Neuromechanical control for hexapedal robot walking on challenging surfaces and surface classification. Robotics and Autonomous Systems, 62(12):1777 - 1789.

Xiong, X., Wörgötter, F., and Manoonpong, P. (2014b). Virtual agonist-antagonist mechanisms produce biological muscle-like functions: An application for robot joint control. Industrial Robot: An International Journal, 41(4):340 - 346.

Yang, C., Ganesh, G., Haddadin, S., Parusel, S., Albu-Schäffer, A., and Burdet, E. (2011). Human-like adaptation of force and impedance in stable and unstable interactions. Robotics, IEEE Transactions on, 27(5):918 -930.

Yu, H., Guo, W., Deng, J., Li, M., and Cai, H. (2013). A cpg-based locomotion control architecture for hexapod robot. In Intelligent Robots and Systems (IROS), 2013 IEEE/RSJ International Conference on, pages 5615-5621. 
Yu, W., Rosen, J., and Li, X. (2011). Pid admittance control for an upper limb exoskeleton. In American Control Conference (ACC), 2011, pages 1124-1129.

Zajac, F. (1989). Muscle and tendon: properties, models, scaling, and application to biomechanics and motor control. Crit Rev Biomed Eng, 17(4):359-411.

Zenker, S., Aksoy, E., Goldschmidt, D., Wörgötter, F., and Manoonpong, P. (2013). Visual terrain classification for selecting energy efficient gaits of a hexapod robot. In Advanced Intelligent Mechatronics (AIM), 2013 IEEE/ASME International Conference on, pages 577-584.

Zill, S., Schmitz, J., and Bschges, A. (2004). Load sensing and control of posture and locomotion. Arthropod Structure \& Development, 33(3):273 - 286. Arthropod Locomotion Systems: from Biological Materials and Systems to Robotics. 


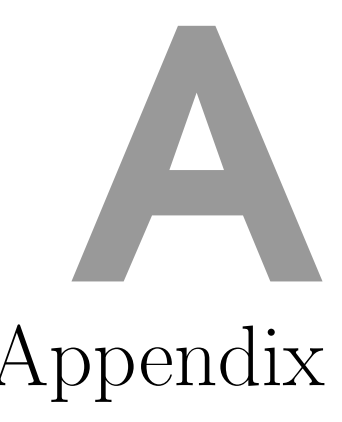

\section{A.1 Joint motion equation}

Substituting Eqs.(3.8), (3.9) and (3.10) into Eq.(3.11), the motion equation of the joint $P$ is given by:

$$
\begin{aligned}
I \ddot{\theta}= & f^{e x t} \sin (\theta) L+r\left[\left(K\left(l_{1}^{P}-l_{0}\right)+D v_{1}^{P}+N_{j} i_{1}\right)\right. \\
& \left.-\left(K\left(l_{2}^{P}-l_{0}\right)+D v_{2}^{P}+N_{j} i_{2}\right)\right] .
\end{aligned}
$$

The lengths of $P E_{(1,2)}$ (i.e., $\left.l_{(1,2)}^{P}\right)$ are equal to the lengths of $M 1$ (i.e., $\left.L_{1}\right)$ and $M 2$ (i.e., $\left.L_{2}\right)$,

$$
l_{1}^{P}=L_{1}, L_{2}=l_{2}^{P} .
$$

In Fig. 3.4, $M 1$ is shortening when $M 2$ is lengthening. Therefore, the relationship between displacements of $M 1$ (i.e., $\left.\Delta L_{1}\right), M 2$ (i.e., $\left.\Delta L_{2}\right)$ and $P E_{(1,2)}$ (i.e., $\Delta l_{(1,2)}^{P}$ ) is given by:

$$
-\Delta l_{1}^{P}=-\Delta L_{1}=\Delta L_{2}=\Delta l_{2}^{P} .
$$

Here we postulate the relationship between displacements $\Delta l_{1}^{P}$ of $P E_{1}, \Delta l_{2}^{P}$ of $P E_{2}$ and the joint angle $\theta$ as (derived by Eqs.(A.2) and (A.3)):

$$
-\left(l_{1}^{P}-l_{0}\right)=-\Delta l_{1}^{P}=\theta r=\Delta l_{2}^{P}=l_{2}^{P}-l_{0},
$$

where $r$ is the radius of the joint $P$. The relationship between velocities $\Delta \dot{l}_{1}^{P}$ of $P E_{1}$, $\Delta \dot{l}_{2}^{P}$ of $P E_{2}$ and the joint velocity $\dot{\theta}$ is given by:

$$
-v_{1}^{P}=-\Delta \dot{l}_{1}^{P}=\dot{\theta} r=\Delta \dot{l}_{2}^{P}=v_{2}^{P} .
$$

Besides, since the motions of $M 1$ and $M 2$ are against each other, their activation intensities $i_{(1,2)}$ are set to:

$$
i_{1}=-i_{2}=0.5
$$

\section{A.2 Notations}

- $F_{6 \times 1}^{e x t}$ is the matrix of the forces, i.e., $F_{6 \times 1}^{e x t}=\left[f_{1}^{e x t}, f_{2}^{e x t}, \ldots, f_{6}^{e x t}\right]^{T}$;

- $F_{6 \times 1}^{\perp}$ is the Hadamard product of $F_{6 \times 1}^{e x t}$ and $\sin \left(\theta 1_{6 \times 1}\right)$; 
- $L_{1}$ is the length of the link between the FTi joint and the end effector of the leg, e.g., $L_{1}=0.115 \mathrm{~m}$;

- $I$ is the inertia of the FTi and CTr joints, i.e., $I=0.5 \times 10^{-3}$;

- $r$ is the radius of the joints, i.e., $r=0.1$;

- $\ddot{\theta} 1_{6 \times 1}$ and $\dot{\theta 1} 1_{6 \times 1}$ are the acceleration and velocity matrices of $\theta 1_{6 \times 1}$;

- $K(1,2)_{6 \times 1}$ and $D(1,2)_{6 \times 1}$ are matrices of the stiffness and damper parameters of $P E_{(1,2)}$, which control the compliance of the FTi and CTr joints.

- $L_{2}$ is the length of links between the CTr and FTi joints, i.e., $L_{2}=0.075 \mathrm{~m}$;

- $\overrightarrow{V 1_{6 \times 1}}$ and $\overrightarrow{V 2_{6 \times 1}}$ are matrices of the displacement vectors of the CTr and FTi joints relating to the forces $f_{1-6}^{e x t}$.

- $\ddot{\theta} 2_{6 \times 1}$ and $\dot{\theta} 2_{6 \times 1}$ are the acceleration and velocity matrices of $\theta 2_{6 \times 1}$.

- $C_{6 \times 1}$ is the matrix of the CTr neuron outputs of the MNN, i.e., $C_{6 \times 1}=\left[N_{7}, N_{8}, \ldots, N_{12}\right]^{T}$.

\section{A.3 Neuromechanical control of the legs}

\section{A.3.1 Swing phase}

When a leg is in a swing phase (i.e., $f_{i}^{e x t}=0, i \in \mathbb{Z}_{[1,6]}$, see Fig. 4.4 (a)), outputs $O_{(i, i+6, i+12)}$ for its TC, CTr and FTi joints receive the outputs of the motor neurons $N_{(i, i+6, i+12)}$ as their inputs.

\section{TC joints}

The TC neuron outputs $N_{1-6} \in[-0.81,0.91]$ are scaled into the TC outputs $O_{1-6} \in$ $[-0.37,0.32]$ :

$$
\begin{aligned}
O_{1-6} & =\frac{N_{1-6}-(-0.81)}{0.91-(-0.81)}(0.32-(-0.37))-0.37 \\
& \approx 0.4 N_{1-6}-0.05
\end{aligned}
$$

\section{CTr joints}

The CTr neuron outputs $N_{7-12} \in[-1,0,1.0]$ are scaled into the CTr outputs $O_{7-12} \in$ $[0.71,1.0]$ :

$$
\begin{aligned}
O_{7-12} & =\frac{N_{7-12}-(-1.0)}{1.0-(-1.0)}(1.0-0.71)+0.71 \\
& \approx 0.15 N_{7-12}+0.86 .
\end{aligned}
$$




\section{FTi joints}

The FTi neuron outputs $N_{13-18} \in[-0.9,1.0]$ are scaled into the FTi outputs $O_{13-18} \in$ $[-0.41,-0.45]$ :

$$
\begin{aligned}
O_{13-18} & =\frac{N_{13-18}-(-0.9)}{1.0-(-0.9)}((-0.45)-(-0.41))-0.41 \\
& \approx-0.02 N_{13-18}-0.43 .
\end{aligned}
$$

\section{A.3.2 Stance phase}

\section{TC joints}

All TC joints are purely controlled by $C E_{(1,2)}$ of the VAAM. $C E_{(1,2)}$ are activated by the outputs $N_{1-6}$ of the modular neural network. The TC neuron outputs $T_{j, 1} \in$ $[-0.81,0.91]$ are scaled into the TC outputs $O_{j} \in[-0.37,0.32]$ (see Fig. 4.6 (a), $\left.j \in \mathbb{Z}_{[1,6]}\right)$ :

$$
\begin{aligned}
O_{j} & =\frac{T_{j, 1}-(-0.81)}{0.91-(-0.81)}(0.32-(-0.37))-0.37 \\
& \approx 0.4 T_{j, 1}-0.05
\end{aligned}
$$

\section{CTr joints}

Each CTr joint is driven by $P E_{(1,2)}$ and $C E_{(1,2)}$ of the VAAM. $C E_{(1,2)}$ are activated by one of the outputs $N_{7-12}$ of the modular neural network (see Fig. 4.6 (a)). The angles $\theta 2_{m, 1}\left(m \in \mathbb{Z}_{[1,6]}\right)$ of the CTr joints are linearly transformed into their outputs $O_{j}$ (see Fig. 4.4). $O_{j}$ are given by $\left(j \in \mathbb{Z}_{[7,12]}\right)$ :

$$
\begin{aligned}
O_{j} & =\frac{\theta 2_{j-6,1}-0.785}{-1.745-0.785}(1-(-1))-1 \\
& \approx-0.8 \theta 2_{j-6,1}-0.38
\end{aligned}
$$

\section{FTi joints}

Each FTi joint is only driven by $P E_{(1,2)}$ of the VAAM (see Fig. 4.6 (a)). The angles $\theta 1_{m, 1}\left(m \in \mathbb{Z}_{[1,6]}\right)$ of the FTi joints can be linearly transformed into their outputs $O_{j}$ (see Fig. 4.4). $O_{j}$ are given by $\left(j \in \mathbb{Z}_{[13,18]}\right)$ :

$$
\begin{aligned}
O_{j} & =\frac{\theta 1_{j-12,1}-(-1.22)}{0.96-(-1.22)}(1-(-1))-1 \\
& \approx 0.92 \theta 1_{j-12,1}+0.12 .
\end{aligned}
$$




\section{A.4 The tripod gaits of AMOS and an insect}

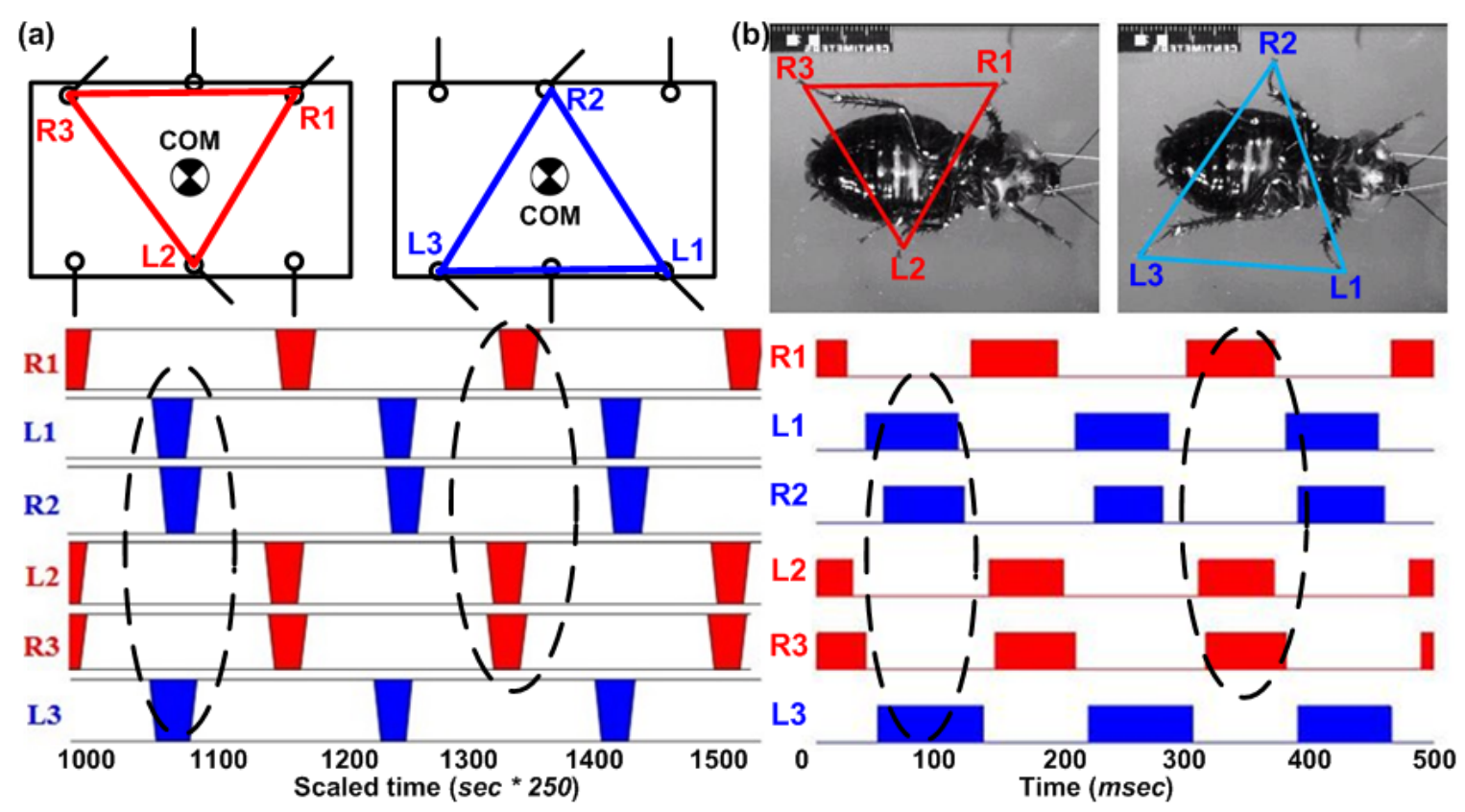

Figure A.1: The tripod gaits of AMOS and an insect. (a) A tripod gait of AMOS. The gait is the slow tripod gait (see Fig. $5.1(\mathrm{~h})$ ) when the modulatory input $S$ of the MNN is set to 0.16 . Note that time here is the scaled time $t$ (i.e., $t=250 *$ second). (b) A tripod gait of an insect on a tether (modified from Ritzmann and Zill (2013)). Red or blue areas indicate no ground contacts during swing phases while white areas refer to ground contacts during stance phases. The tripod gaits mean that the right front (i.e., R1) and hind (i.e., R3) legs move as a unit with the left (i.e., L2) middle leg. This tripod alternates between swing and stance phases with another tripod that consists of the remaining three legs (i.e., L1, R2, and L3). 


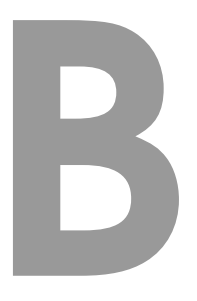

\section{Curriculum Vitae}

\section{Xiaofeng Xiong}

Research Assistant at the Bernstein Center for Computational Neuroscience

Georg-August-Universität Göttingen

III Physikalisches Institut - Biophysik

Friedrich-Hund Platz 1

37077 Gttingen

Date and place of birth: 12 March 1983

Hainan, China

Citizenship: China

E-mail: Xiaofeng.Xiong@phys.uni-goettingen.de

Tel.: + +49 (0) $55139-12703$

\section{EDUCATION}

2011 May - 2015 June

PhD Student at the Department of Computer Science Georg-August-Universität Göttingen

Germany

2005 September - 2008 July M.Sc. in Mechatronic Engineering

Beijing University of Technology

Beijing, China

2001 September - 2005 July B.Sc. in Electronic and Information Engineering

Jilin University

Jilin, China

\section{AWARDS and SCHOLARSHIP}

- 2013 The Industrial Robot Innovation Award for practical innovation in the field of robotics (Highly Commended) at 16th International Conference on Climbing and Walking Robots and the Support Technologies for Mobile Machines (CLAWAR). 
- 2011 Emmy Noether Ph.D. scholarship, German Research Foundation (DFG).

\section{RESEARCH INTERESTS}

Neuromechanical control

Sensorimotor learning

Biomechanics

Biorobotics

Variable compliance control

Legged robots

\section{LIST OF PUBLICATIONS}

\section{Journal Papers}

- Xiong, X., Wörgötter, F., and Manoonpong, P. (2014). Neuromechanical control for hexapedal robot walking on challenging surfaces and surface classification. Robotics and Autonomous Systems, 62(12):1777 - 1789.

- Xiong, X., Wörgötter, F., and Manoonpong, P. (2014). Virtual agonist-antagonist mechanisms produce biological muscle-like functions: An application for robot joint control. Industrial Robot: An International Journal, 41(4):340 - 346.

\section{Conference Papers}

- Xiong, X., Wörgötter, F., and Manoonpong, P. (2013). A neuromechanical controller of a hexapod robot for walking on sponge, gravel and snow surfaces. In Advances in Artificial Life. Proceedings of the 11th European Conference on Artificial Life ECAL 2013, pages 989-996, Taormina, Italy.

- Xiong, X., Wörgötter, F., and Manoonpong, P. (2013). A simplified variable admittance controller based on a virtual agonist-antagonist mechanism for robot joint control. In Proc. Intl Conf. on Climbing and Walking Robots CLAWAR, pages 281-288, Sydney, Australia. 